https://theses.gla.ac.uk/

Theses Digitisation:

https://www.gla.ac.uk/myglasgow/research/enlighten/theses/digitisation/

This is a digitised version of the original print thesis.

Copyright and moral rights for this work are retained by the author

A copy can be downloaded for personal non-commercial research or study, without prior permission or charge

This work cannot be reproduced or quoted extensively from without first obtaining permission in writing from the author

The content must not be changed in any way or sold commercially in any format or medium without the formal permission of the author

When referring to this work, full bibliographic details including the author, title, awarding institution and date of the thesis must be given

Enlighten: Theses

https://theses.gla.ac.uk/

research-enlighten@glasgow.ac.uk 


\title{
Comparison of the Effects of Trypsin and Chymotrypsin on Thylakoid Membrane Function.
}

\section{By}

Christine Anne Raines, B.Sc.

A thesis submitted for the degree of Ph.D.

\author{
University of Glasgow \\ Department of Botany.
}

November 1985 . 
ProQuest Number: 10907158

All rights reserved

INFORMATION TO ALL USERS

The quality of this reproduction is dependent upon the quality of the copy submitted.

In the unlikely event that the author did not send a complete manuscript and there are missing pages, these will be noted. Also, if material had to be removed, a note will indicate the deletion.

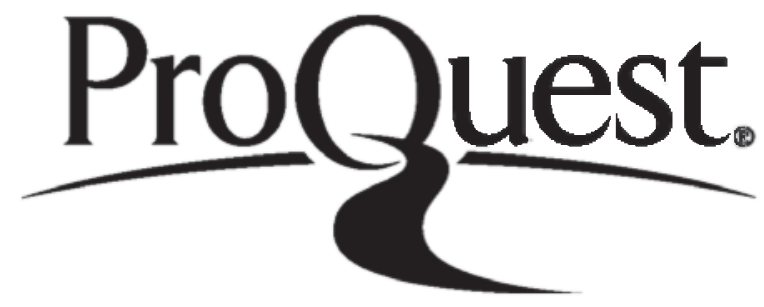

ProQuest 10907158

Published by ProQuest LLC (2018). Copyright of the Dissertation is held by the Author.

All rights reserved.

This work is protected against unauthorized copying under Title 17, United States Code Microform Edition (C) ProQuest LLC.

ProQuest LLC.

789 East Eisenhower Parkway

P.O. Box 1346

Ann Arbor, Ml $48106-1346$ 


$\begin{array}{lr} & \frac{\text { Page }}{\text { Dedication }} \\ \text { Acknowledgements } & \text { I } \\ \text { Abstract } & \text { II } \\ \text { Abbreviations } & \text { III } \\ \end{array}$

Chapter 1: Introduction.

1.1 The Importance of Photosynthesis 1

1.2 The Overall Process 3

1.3 Light Absorption and Energy Trapping 5

1.3.1 The Photosynthetic Pigments 5

1.3.2 The Photosynthetic Unit 6

1.3.2.1 The Antenna Complexes 8

1.3.2.2 The Reaction Centre Complexes 8

1.4 Electron Transport 10

1.4.1.1 Linear Electron Transport 10

1.4.1.2 Cyclic Electron Transport 12

$\begin{array}{lll}1.4 .2 & \text { Donors to PSII } & 14\end{array}$

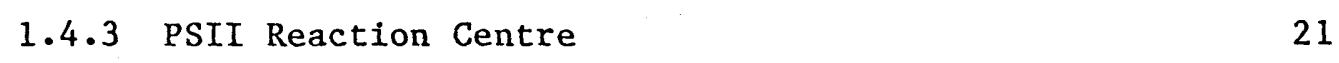

1.4.4 The Plastoquinone Pool 24

1.4.5 Intersystem Electron Carriers 25

1.4.5.1. The Reiske-iron Centre 25

1.4.5.2 Cytochrome b $563 \quad 26$

1.4.5.3 Cytochrome $\underline{f} \quad 26$

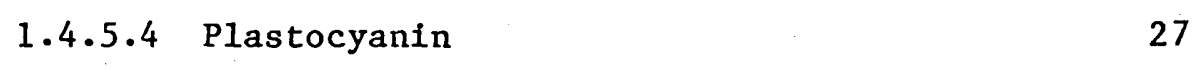

1.4.6 PSI Reaction Centre . 28

1.4.7 Q- and b- Cycles of Electron Transport 30

1.5 Photophosphorylation 33

1.5.1 The Chemiosmotic Theory i 34 
1.5.1.1 Energetics of ATP Synthesis 35

1.5.1.2 Stoichiometry of $\mathrm{H}^{+} / \operatorname{ATP} \quad 36$

$\begin{array}{ll}\text { 1.5.2 The ATPase Enzyme Complex } & 37\end{array}$

1.5.2.1 Functional Properties 37

1.5.2.2 Nucleotide Binding 39

1.5.2.3 Molecular Mechanisms $\quad 40$

$\begin{array}{lll}1.6 & \text { The Thylakoid Membrane } & 41\end{array}$

1.6.1 The Morphology 41

1.6.1.1 Regulation of Energy Redistribution $\quad 42$

1.6.2 The Molecular Structure 43

1.6.2.1 Transverse Asymmetry 44

1.6.2.2 Lateral Asymmetry $\quad 45$

1.6.3 The Protein Complexes 46

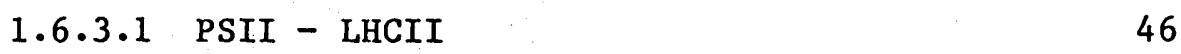

$\begin{array}{lll}1.6 .3 .2 & \text { PSI - LHCI } & 47\end{array}$

1.6 .3 .3 Cyt $\underline{\mathrm{b}} / \underline{\mathrm{f}} \quad 49$

1.6.3.4 ATPase Enzyme $\quad 50$

1.7 Proteolysis of Membranes 51

1.8 Experimental Rationale $\quad 53$

1.9 Theory of Experimental Methods $\quad 54$

1.9.1 Flash Absorption Spectrophotometry 54

1.9.1.1 The Electrochromic Bandshift at 515nm
$(\triangle \mathrm{A} 515)$

1.9.1.2 Light Induced Shift in the Absorption
of Oxonol VI $(\triangle A$ 575)

$\begin{array}{ll}\text { 1.9.2 Chlorophyll a Fluorescence } & 61\end{array}$

Chapter 2: Materials and Methods

2.1 Growth Conditions $\quad 64$

2.2 Isolation of Chloroplasts 64 
2.3 Determination of Chlorophyll Concentration 64

2.4 Experimental Procedures $\quad 65$

2.4.1 Enzyme Incubation Procedure 65

2.4.2 Enzyme Assays $\quad 66$

$\begin{array}{ll}2.4 .3 & \text { Protein Assay }\end{array}$

2.4.4 PSII - Blocked Chloroplasts 68

2.5 Measurement of $\mathrm{O}_{2}$ Evolution $\quad 68$

2.5.1 The Steady-State $\mathrm{O}_{2}$ Electrode 68

2.5.2 The Flash $0_{2}$ Electrode 69

2.6 Measurement of Photophosphorylation and Hydrolysis 71

2.7 Flash Induced Spectrophotometry 72

2.7.1 Measurement of the Flash-induced Electrochromic
Bandshift ( $\triangle \mathrm{A} 515)$

2.7.2 Measurement of Flash-induced Oxonol VI $\begin{array}{ll}\text { Absorption Changes } & 74\end{array}$

2.8 Measurement of Fluorescence $\quad 74$

$\begin{array}{lll}2.8 .1 & \text { Chlorophyll a fluorescence } & 74\end{array}$

2.8.29 - Aminoacridine Fluorescence 75

2.9 Coupling Factor Isolation and Purification 75

2.9.1 Isolation of Chloroplasts 75

2.9.2 Extraction of Coupling Factor : 76

$\begin{array}{lll}2.9 .3 & \text { Purification of Coupling Factor } & 77\end{array}$

$\begin{array}{lll}2.9 .4 & \text { Polyacrylamide Gel Electrophoresis } & 77\end{array}$

Chapter 3: The Effects of Trypsin and Chymotrypsin on Electron Transport in Isolated Thylakoids

$\begin{array}{ll}3.1 \cdot \text { Introduction } & 78\end{array}$

$\begin{array}{lll}3.2 & \text { Results } & 79\end{array}$

$\begin{array}{lll}3.3 & \text { Discussion } & 81\end{array}$

3.3.1 Effects on Uncoupled Electron Transport 81 
Chapter 4: The Effects of Trypsin and Chymotrypsin on the Function of the ATPase Enzyme Complex

4.1 Introduction $\quad 83$

$\begin{array}{lll}4.2 & \text { Results } & 84\end{array}$

4.2.1 The Effects of Dark Adaption and Light Incubation on the Electrochromic Bandshift $\quad 84$

4.2.2 Changes in the Electrochromic Bandshift Induced by Treatment of Thylakoids with Trypsin or Chymotrypsin

4.2.3 The Effects of Adenine Nucleotides on the Electrochromic Bandshift in the Thylakoids Pre-treated with Dithiothreitol, Trypsin and Chymotrypsin

4.2.4 The Effect of Tentoxin on the Ability of ATP to Decrease the Half-time of decay of the Electrochromic Bandshift, in Thylakoids Pre-treated with Dithiothreitol, Trypsin and Chymotrypsin

4.2.5 The Effect of Trypsin and Chymotrypsin on ATP Hydrolysis

4.2.6 The Effect of Trypsin and Chymotrypsin on ATP Synthesis

4.2.7 The Effect of ATP on 9-Aminoacridine Fluorescence in Thylakoids Pre-treated with Trypsin and Chymotrypsin

4.2.8 The Effects of Trypsin and Chymotrypsin on the Subunits of the Coupling Factor

4.3.1 Uncoupling of the Thylakoid Membrane Caused by Trypsin and Chymotrypsin Treatment

4.3.2 Trypsin and Chymotrypsin : Effects on ATP Hydrolysis

4.3.3 The ATPase Complex : Sensitive Sites on $\mathrm{CF}_{1}$ 
Chapter 5: The use of Oxonol VI as a Membrane Probe to Investigate the Effects of Trypsin and Chymotrypsin on the Electrochromic Bandshift Indicating-pigments

5.1 Introduction

102

5.2 Results

5.2.1 The Light-minus-dark Difference Spectrum of Chloroplasts Treated with Trypsin and Chymotrypsin

5.2.2 Optimum Oxonol VI Concentration

104

5.2.3 The Effects of Trypsin and Chymotrypsin on the Oxonol VI Absorption Change and on the Electrochromic Bandshift

5.2.4 A Comparison Between the Effects of Gramicidin and DCMU on the Electrochromic Bandshift and on the Oxonol VI Absorption Change

\subsection{Discussion}

Chapter 6: Effects of Trypsin and Chymotrypsin on PSII Mediated Reactions

6.1 Introduction

6.2 Results

6.2.1 The Effects of Trypsin and Chymotrypsin on the Electrochromic Bandshift in Thylakoids Resuspended in Low- or High-salt Media

6.2.2 The Effects of Trypsin and Chymotrypsin on the $\mathrm{Mg}^{2+}$-induced Chlorophyll Fluorescence Rise

6.2.3 The Effects of Trypsin and Chymotrypsin on the Electrochromic Bandshift Induced by PSI Alone

6.2.4 The Relative Flash Yield of Oxygen as a Function of Light Intensity in Enzyme Treated Thylakoids

6.2.5 Induction of Chlorophyl1 Fluorescence in DCMUBlocked Thylakoid Membranes which have been Treated with Trypsin or Chymotrypsin

6.2.6 The Effects of Trypsin and Chymotrypsin on the Rate of Light-induced Oxygen Evolution in Uncoupled Thylakoid Membranes 
Chapter 7: Discussion

$\begin{array}{lll}7.1 & \text { Introduction } & 118\end{array}$

$\begin{array}{lll}7.1 .1 & \text { Electron Transport } & 118\end{array}$

$\begin{array}{ll}\text { 7.1.2 The ATPase Complex } & 119\end{array}$

$\begin{array}{lll}\text { 7.1.3 PSII - Mediated Reactions } & 121\end{array}$

7.2 Conclusions 122

7.2.1 Summary table of results $123 a$

References $\quad 124$

$\begin{array}{ll}\text { Additional references } & 125\end{array}$ 
DEDICATION.

This work is dedicated to Malcolm for his continued patience, consideration and understanding. 


\section{ACEKNOWLEEDGEMENTS}

I would like to convey my sincere thanks and appreciation to my supervisor Dr. M.F. Hipkins for his unending and invaluable support throughout my three years of study. I am also grateful, firstly to Professor M.B. Wilkins and laterly to Professor J.R. Hillman for the use of the facilities in the Botany Department. In addition I wish to thank Dr. R. J. Cogdell and Dr. J. Valentine for encouragement, helpful discussions and advice. Further thanks are extended to Dr. V. Math, E. Robertson and N. Tait for technical assistance and to Margaret Winship who typed this manuscript.

I wish to mention the postgraduate students at the Garscube Research Laboratories with whom it has been a pleasure to work; in particular Candy Paige and Dennis Aw whose friendship has made these past three years memorable.

I gratefully acknowledge the financial support from the Glasgow University Scholarship.

Final thanks go to my parents to whom I am eternally indebted for all their support and encouragement throughout the years: 
Abstract.

The thylakoid membranes are the functional units upon which the photosynthetic energy-tansducing reactions occur. There is a strict relationship between the structure of these membranes and their functional capabilities; this is because the components of the photosynthetic electron-transport chain are bound to proteins in the membrane in

a specific manner. Functional changes can be induced by using protediytic enzymes to alter the structure of the proteins. Two enzymes, trypsin (which digests bonds involving arginine and lysine) and chymotrypsin (which digest bonds involving tryptophan, tryosine and phenylalanine) have been used to investigate the functions of the thylakoid membrane. The rationale was that because these enzymes have different specificities for the bonds which they attack they may induce different functional changes in the membrane. The effects of these enzymes are compared.

Measurements of 1 ight-induced oxygen evolution rates in the presence of exogenous electron acceptors showed that both trypsin and chymotrypsin altered the electron transport reactions in a number of ways. At higher concentrations both trypsin and chymotrypsin treatment of thylakoids caused the rate of electron transport to be dependent on the type of electron acceptor present and rendered electron transport insensitive to 3-(3,4-dichloropheny1)-1,1 dimethylurea which inhibits photosystem II. These effects of trypsin have previously been, shown to be due to partial digestion of a $32 \mathrm{kDa}$ protein on the acceptor side of photosystem II. Thus chymotrypsin may also affect this polypeptide. At lower concentrations 
trypsin, and to a lesser extent chymotrypsin, stimulated the rate of electron transport. This suggested that both of these enzymes tend to uncouple the thylakoid membranes.

Trypsin and chymotrypsin altered the flash-induced field indicating absorption change measured at $520 \mathrm{~nm}$ (the electrochromic bandshift) in two ways: 1) the half-time of decay was decreased suggesting increased trans-membrane ion flux and 2) the extent was reduced. The decrease in the half-time of the decay of the electrochromic bandshift was correlated with uncoupling, by trypsin and to a lesser extent by chymotrypsin, of the thylakoid membrane. This proposal was supported by phosphorylation measurements which showed that both trypsin and chymotrypsin can, at least partially, inhibit ATP synthesis. But after brief trypsin incubation in the light a stimulation in the rate of phosphorylation and oxygen evolution (water to methyl viologen) was evident. It is possible that trypsin affects the $\varepsilon$ sub-unit, believed to be an inhibitor of ATPase function, however, no evidence to support this proposal was seen in polyacrylamide gel electrophoresis analysis of the ATPase.

ATP hydrolysis was stimulated by both trypsin and chymotrypsin and this was tentoxin sensitive. When ATP was added to thylakoids in which ATP hydrolysis was active the half-time of decay of the electrochromic bandshift was decreased significantly. These data suggest that the decay of the electrochromic bandshift is accelerated by trypsin and chymotrypsin because these enzymes stimulate ATP hydrolysis; thus in the presence of ATP in the dark, an appreciable trans-membrane electrochemical potential gradient of 
protons exists. When a flash-induced field is imposed on top of the steady state electrochemical potential gradient, the driving forces for ion flux through the membrane are larger than in the control, hence the electrochromic bandshift decays more rapid1y.

The reduction in the extent of the electrochromic bandshift caused by trypsin or chymotrypsin was investigated using an extrinsic membrane-potential probe, oxonol VI. The flash-induced absorbance change of oxonol VI proved to be more sensitive than thet electrochromic bandshift to enzyme treatment of the thylakoids. It was shown that oxonol VI is best used as an indicator of membrane potential under conditions where the potential is changing slowly.

Trypsin was shown to decrease the amplitude of the electrochromic bandshift more markedly in thylakoids treated in a high-salt medium as compared to lowsalt samples, whilst chymotrypsin had a similar effect in either media. The data from measurements of the electrochromic bandshift induced by PSI alone and from measurements of the $\mathrm{Mg}^{2+}$ - induced fluorescence increase suggested that trypsin treatment of thylakoids in high-salt media reduced the amplitude of the bandshift by removing a portion of light harvesting complex associated with PSII. This polypeptide has previously been implicated in the control of energy redistribution between the two photosystems; its removal leads to a reduced excitation of photosystem II and enhanced excitation of photosystem I. However this explanation does not account for the effect of chymotrypsin or trypsin in low-salt riedium for which there are three possibilities: 1) removal of light harvesting complex associated with the photosystems; 2) a direct effect on the electrochropic 
bandshift sensing-pigments and 3 ) a direct effect on the functions of the reaction centres. Measurements of flash-induced oxygen yield as a function of flash intensity suggest that chymotrypsin has no effect on the light harvesting pigments associated with photosystem II. Moreover chlorophyll fluorescence induction curves indicated that neither chymotrypsin nor trypsin in low-salt affected the primary photochemical reactions at the reaction centre of photosystem II. But measurements of steady-state light-induced oxygen evolution suggest that both trypsin and chymotrypsin redueed the rate of electron transport even at low enzyme concentrations. In view of this result an effect on the efficiency of the photosystem II cannot be ruled out but it is still possible that chymotrypsin and trypsin (in low-salt media) affect the size of the electrochromic bandshift by removal of some of the sensing pigments. 


\begin{tabular}{|c|c|}
\hline RUBP & Ribulose Bisphosphate Carboxylase \\
\hline Ch1 & Chlorophy11 \\
\hline PSII/I & Photosystem II or I \\
\hline M & Water Evolving Complex \\
\hline $\mathrm{Z}$ & Primary Donor to P680 of PSII \\
\hline P680 & Primary Donor of PSII \\
\hline Qa & Primary Acceptor of PSII \\
\hline $\mathrm{Qb}$ & Secondary Acceptor of PSII \\
\hline Cyt $\underline{f}$ & Cytochrome $\underline{\underline{E}}$ \\
\hline P700 & Primary Donor of PSI \\
\hline $\mathrm{x}$ & Primary Acceptor of PSI \\
\hline Fe- S A/B & Secondary Acceptors of PSI \\
\hline ATP & Adenosine Tri-Phosphate \\
\hline DCMU & 3-(3,4-dichloropheny 1$)-1,1$ dimethylurea \\
\hline NADP & Nicotinamide Adenine Dinucleotide Phosphate \\
\hline ESR & Electron Spin Resonance \\
\hline NMR & Nuclear Magnetic Resonance \\
\hline XAES & X-ray Absorption Edge Spectroscopy \\
\hline EXFAS & Extended X-ray Absorption Fine Structure \\
\hline EPR & Electron Paramagnetic Resonance Spectra \\
\hline$Q_{\mathrm{H}}$ & High Redox Potential Q \\
\hline QL & Low Redox Potential Q \\
\hline $\mathrm{ADP}$ & Adenosine Diphosphate \\
\hline$C F_{1}, C F_{0}$ & Coupling Factor, extrinsic and intrinsic portions \\
\hline DTT & Dithiothreitol \\
\hline LHCP & Light Harvesting Chlorophy11 Protein \\
\hline$\triangle \mathrm{A} 515$ & Light-induced Absorption Change at $515 \mathrm{~nm}$ \\
\hline DCPIP & 2,6-dichlorophenolindophenol \\
\hline TMPD, & $N, N, N^{\prime}, N$-tet ramethylphenyldiamine \\
\hline
\end{tabular}


CHAPTER ONE - INTRODUCTION. 


\subsection{THE_IMPORTANCE OF PHOTOSYNTHESIS.}

Photosynthesis is the process by which radiant energy from the sun is converted into chemical energy by green plants, algae and some bacteria. The importance of this process cannot be overstated as all life on this planet, with the exception of chemotrophic bacteria, is dependent upon photosynthesis as a source of food; moreover green plant photosynthesis maintains the level of $\mathrm{O}_{2}$ in the atmosphere. In addition to these indispensable functions plants are a source of fuel and fibre.

The photosynthetic process has been intensively studied and this has yielded a large amount of information on the mechanism and more recently on the structures involved. As yet little of this knowledge has been applied to trying either to improve or mimic photosynthesis. However, it is now possible to speculate about the future and look at what applications may be made of this basic research. Two areas currently being investigated are: 1) the production of totally artificial systems. A recent paper (Calvin, 1983) showed that it is now possible to produce an artificial system capable of light absorption and charge separation, the first two steps in the natural process. The next stage is to extend this model to allow it to store the absorbed energy which could subsequently be used to do work. The final aim of this work would be to create an alternative energy source to that of fossil fuel; and 2) the manipulation of genes which code for proteins that are important in photosynthesis. Two proteins which are targets for manipulation are the 
$32 \mathrm{KDa}$ protein implicated in herbicide binding, and RUBP carboxylase, the key enzyme in $\mathrm{CO}_{2}$ fixation (Grierson and Covey, 1984). The objective of this research is to improve the natural photosynthetic system thus hopefully increasing the yield. 


\subsection{THE OVERALL PROCEESS.}

The overall photosynthetic reaction involves the conversion of $\mathrm{CO}_{2}$ and $\mathrm{H}_{2} \mathrm{O}$ into carbohydrates which can be used subsequently as a source of energy.

The reaction in green plants can be written thus:

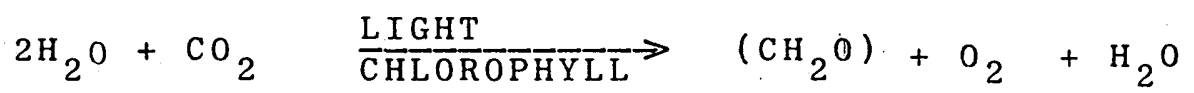

Sulphur and non sulphur photosynthetic bacteria can fix $\mathrm{CO}_{2}$ but no $\mathrm{O}_{2}$ is evolved:

$$
\begin{aligned}
& 2 \mathrm{H}_{2} \mathrm{~S}+\mathrm{CO}_{2} \frac{\mathrm{B}}{\mathrm{L}} \frac{\mathrm{Ch}}{\mathrm{I}} \frac{\mathrm{I}}{\mathrm{G}} \overrightarrow{\mathrm{T}} \cdot\left(\mathrm{CH}_{2} \mathrm{O}\right)+2 \mathrm{~S}+\mathrm{H}_{2} \mathrm{O} \text { (SULPHUR ) }
\end{aligned}
$$

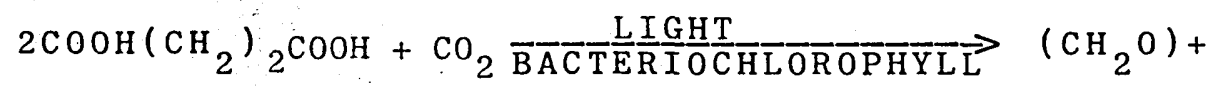

$$
\begin{aligned}
& 2 \mathrm{COOH}(\mathrm{CH}){ }_{2} \mathrm{COOH}+\mathrm{H}_{2} \mathrm{O}(\mathrm{NON}-\mathrm{SULPHUR})
\end{aligned}
$$

van Neil (1941) compared the reactions in bacteria with those in higher plants and, from the similarities between the reactions, he suggested that in each case $\mathrm{CO}_{2}$ was reduced at the expense of an oxidisable substrate. A general reaction describing the process can be represented thus:

$2 \mathrm{H}_{2} \mathrm{~A}+\mathrm{CO}_{2} \longrightarrow\left(\mathrm{CH}_{2} \mathrm{O}\right)+\mathrm{H}_{2} \mathrm{O}+2 \mathrm{~A}$

This proposal implied that in green plants $0_{2}$ was produced from the oxidation of water and that $\mathrm{CO}_{2}$ was reduced to form carbohydrate. Experiments, of Hill (1937) supported this: illumination of isolated chloroplasts in the presence of a reducing agent resulted in the evolution of $\mathrm{O}_{2}$ without the occurrence of $\mathrm{CO}_{2}$ fixation. Studies, using the heavy isotope of oxygen $\left({ }^{18} \mathrm{O}\right)$ to discriminate between $\mathrm{H}_{2} \mathrm{O}$ and $\mathrm{CO}_{2}$ 
as the source of $\mathrm{O}_{2}$, also suggested that $\mathrm{H}_{2} \mathrm{O}$ was the source of $0_{2}$ (Ruben et al, 1941).

Photosynthesis can be divided into two reactions known as the light reaction and the dark reaction. The former involved the photolysis of water, producing hydrogen ions and electrons which then participate in the formation of ATP and NADPH which are the energy source for $\mathrm{CO}_{2}$ reduction in the dark reaction. The dark reaction (more correctly the non-light requiring reaction) involves a complex cycle of enzyme driven reactions. The biochemical steps involved in carbohydrate formation were worked out by Bassham et al (1954) using radio-isotopes and auto-radiography. They showed that $\mathrm{CO}_{2}$ is incorporated in a reaction with ribulose bisophosphate (RUBP) giving two molecules of 3phosphoglyceric acid. This compound then undergoes several interconversions. (from $\mathrm{C}_{3}$ to $\mathrm{C}_{7}$ carbon compounds) using ATP and NADPH generated in the light reactions to regenerate RUBP. These reactions take place in organelles called chloroplasts. It is a structure bounded by a double membrane which encloses a proteinaceous stroma and an inner continaous membrane system which forms internal closed structures called thyakoid membranes (Fig. 1). The light reactions take place on the thylakoid membranes while the site for the 'dark reactions' is the stroma. 


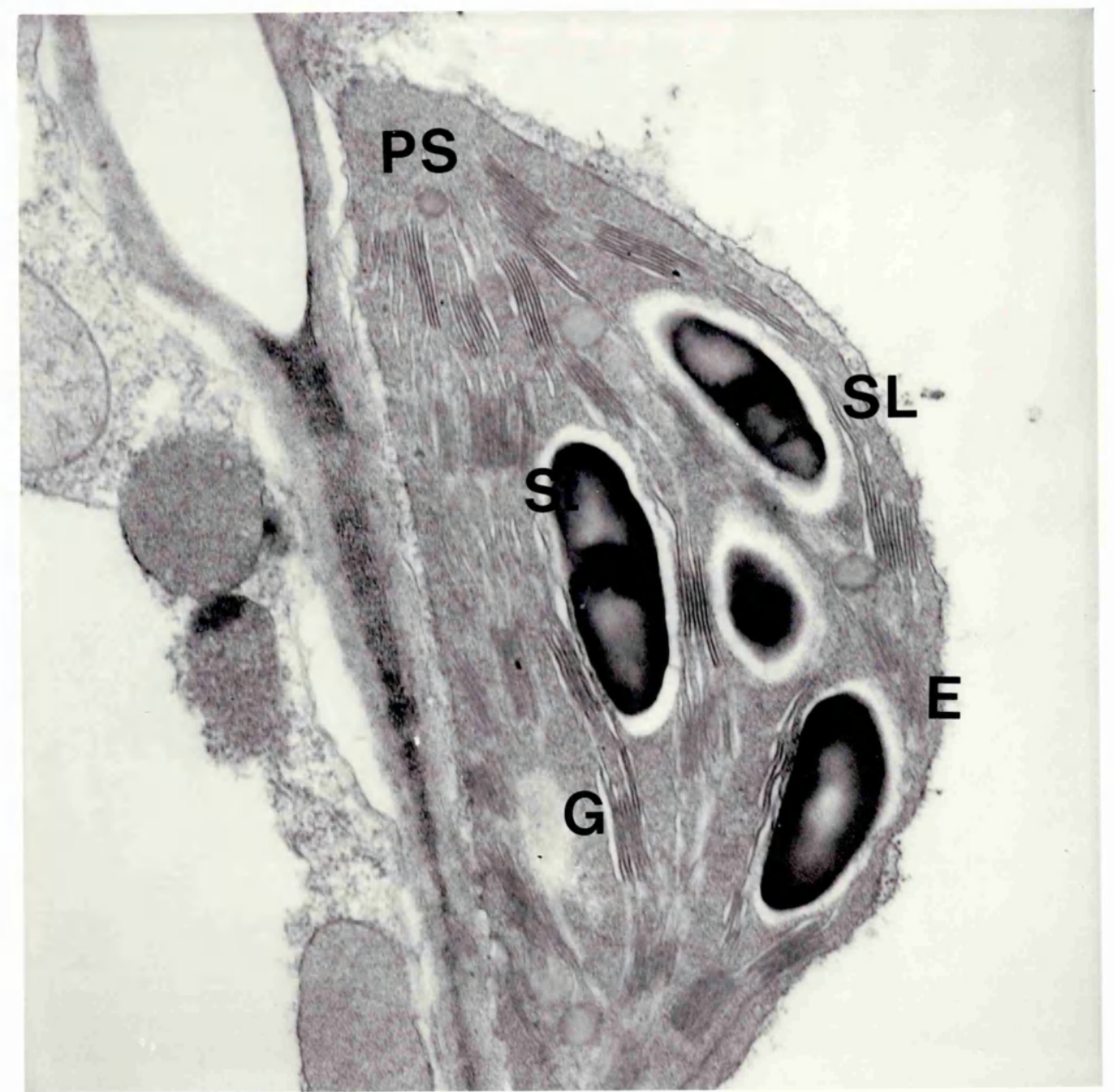

\section{FIGURE 1.}

An electron micrograph of a chloroplast. This organelle is bounded by a double membrane called the outer envelope (E), which encloses a proteinaceous stroma (PS) and the thylakoid membranes. Two structural groups of thylakoid membranes are visible: appressed regions where the membranes form stacks called grana $(G)$, the grana are interconnected by non-appressed membranes called stromal lamellae (SL). The large black objects are starch grains (St).

Magnification 154000. Isolated from Phaseolus vulgaris. 


\subsection{LIGHTAABSORPTION AND ENERGY TRAPPING.}

\subsubsection{The Photosynthetic Pigments.}

The first step in the photosynthetic process is the absorption of light by pigment molecules which are situated in the thylakoid membranes. In green plants the most important pigments are the chlorophyl1s. These are either involved directly in the photochemical reactions (chlorophyll a) or as accessory light-harvesting pigments (chlorophylla and b) In addition to the chlorophylls the other major pigments are the carotenoids.which have two functions 1) 1 ight absorption and 2) a protective role in dissipating superoxide or singlet oxygen radicals which are formed by the overexcitation of chlorophyll in the presence of oxygen.

The photosynthetic pigments in higher plants absorb light in the visible region of the electromagnetic spectrum i.e. between 400 and $700 \mathrm{~nm}$. The absorption spectra of Chla, Chlb and carotenoids in organic solvent: shows that each of these pigments has different wavelengths of maximum absorption. Comparing the absorption spectrums of the photosynthetic pigments with the action spectrum for photosynthesis indicates that there is energy transfer between these pigments (Clayton, 1965) wavelengths where chlorophyll absorbs only weakly photosynthetic activity is at a similar level as at wavelengths where strong absorption by chlorophyll occurs. This system ensures that light absorption occurs right across the spectrum thereby helping to maximise the efficiency of photosynthesis. The absorption spectra so far discussed concern the 
photosynthetic pigments in organic solvents; they display different absorption characteristics in vivo. This difference is brought about because in vivo the pigments are non-covalently bound to proteins in the membrane (Thornber, 1975). This association maintains the pigments in a specific orientation, both within the membrane and in relation to each other and is important for the efficient functioning of these components (see 1.6 ).

\subsubsection{The Photosynthetic Unit.}

The photosynthetic pigments can be divided into two functional groups: 1) the antenna complexes, comprising the majority of the pigments and whose role is 1 ight absorption; 2) the reaction centre complexes which are made up of specialised chlorophyil-protein complexes and which are responsible for trapping the energy received from the antenna complexes. Therefore the majority of the pigments serve as a light gathering funnel which channels the absorbed energy into a small number of energytrapping reaction centres. The concept that chlorophyll molecules could co-operate with each other was recognised first by Emerson and Arnold (1932) who proposed the existence of the photosynthetic unit. Prior to this it was believed that each chlorophyllmolecule was involved in photochemistry (clayton, 1965). In its original form this unit was defined as the number of chlorophyll molecules required to reduce one molecule of $\mathrm{CO}_{2}$ : this was shown by Emerson and Arnold to be about $2400 \mathrm{chl}$. This number has now been modified to take account of new information which has been gained about the mechanism of photosynthesis. Before one molecule of oxygen can be evolved the absorption of four quanta of 1 ight is required (see section 1.4.2) 
therefore each of these would require the co-operation of 600 chlorophyll molecules (2400/4). Further experiments by Emerson (1958) showing the red drop and enhancement effects resulted in the discovery that higher plants had two photosystems. These are now known as photosystem II (PSII) and photosystem I (PSI) and exhibit light-induced bleaching at $680 \mathrm{~nm}$ and $700 \mathrm{~nm}$ respectively. The implication of this discovery was that a minimum of eight quanta are required to reduce one molecule of $\mathrm{CO}_{2}$. Therefore the original number of 2400 can be altered to 300 $(2400 / 8)$; this giving the number of chlorophyll molecules associated with each photosystem both of which perform one photochemical reaction on the absorption of one quanta of 1 ight.

This model of the photosynthetic unit will probably have to be altered in the near future because of recent experimental findings: 1) the ratio of PSII: PSI is not one (Melis and Brown, 1980); 2) the two photosystems are spatially separated along the lateral plane of the membrane (Anderson, 1984;Barber, 1984). In addition when the photosynthetic unit was first conceived little was known about the molecular structure of the photosynthetic membrane, therefore it was not known if this unit was a structural or statistical entity. In recent years it has been possible to isolate light harvesting pigment complexes and reaction centres from higher plants (Anderson and Andersson, 1982) and bacteria (Clayton, 1980). Hence in bacteria it is probably valid to look upon the photosynthetic unit as a structural unit. However in higher plants the picture is not as well resolved and it appears that antenna molecules can move between PSII and PSI ( see section 1.6.1.1). 
1.3.2.1 The Antenna Complexes.

The function of the antenna pigments is to increase the cross-sectional area, and wavelength span of light absorption and to pass this energy on to the reaction centres. Absorption of light by the antenna molecules results in the raising of electrons from the ground to the first or second excited singlet state. Once absorbed this energy is transferred from one antenna molecule to the next via resonance transfer until it reaches the reaction centre. A number of fates can befall this energy: it can be 1) lost in radiationless-deexcitation (emitted as heat);

2) lost as fluorescence or 3 ) used to drive the photochemical reactions in the reaction centres.

1.3.2.2 The Reaction Centre.

Photosystems. I and II each contain the reaction centres within which the photochemical reactions take place. The first direct evidence of photochemical charge separation in reaction centres came when Duysens (1952) observed the reversible light-induced bleaching of P870 in purple bacteria. Later Kok (1956) observed these effects in chloroplasts where a bleaching of P700 was evident.

The energy, passed onto the reaction centres from the antenna pigments, is 'trapped' in the form of oxidising and reducing equivalents. The reactions occurring within the photosystems can be generalised as shown below:

$\mathrm{DPA} \longrightarrow \mathrm{DP} * \mathrm{~A} \longrightarrow \mathrm{DP}^{+} \mathrm{A}^{-} \longrightarrow \mathrm{D}^{+} \mathrm{PA}^{-}$ 
Where $P$ is the primary donor, A the primary acceptor and $D$ the secondary donor which restores $P$ to its original form. These reactions result in the stabilization of charge which creates a redox potential difference between the acceptor and donor of each of the two photosystems. Electrons are then able to flow down the redox potential difference from PSII to PSI. 


\subsection{ELECTRON TRANSPORT.}

\subsubsection{Linear E1ectron Transport.}

The flow of electrons from water to NADP ${ }^{+}$is known as linear electron transport. A scheme describing this linear flow was first outlined by Hill and Bendall (1960); they suggested that the two light reactions co-operate in series and are linked by a system of electron carriers. Evidence in support of this ' $Z$ ' scheme has come from a number of sources one of the most important being the experiments of Duysens et a1 (1961). Using redialgae, Duysens showed the antagonistic effects of light, selectively absorbed by PSII (562nm) or PSI (680nm), on the oxidation state of a cytochrome situated in the thylakoids membranes of the algae. In addition, the use, of mutants of an algae chlamydomonas reinhardii, lacking in one orcothersof the electron transport components (Levine, 1969) lent support to this scheme.

The trapping of energy by PSII results in the formation of a weak reductant, and a strong oxidant which is capable of splitting water. In PSI a weak oxidant, and a strong reductant capable of reducing NADPt, are formed. The formation of these redox pairs at the reaction centres creates a

redox potential differencewhich initiates the flow of

electrons down the electron transport chain. This chain can be divided into three sections: 1) from water through $M$ and $z$ to the primary donor of PSII (P680, ChlaII); 2) from the primary acceptor of PSII 
(Q) to $Q b$, thence to the plastoquinone pool, the Reiske iron-suplhur centre, cyt $f$, plastocyanin and finally the primary donor of PSI (P700); and 3 ) from the primary acceptor of PSI (X, $\left.A_{2}\right)$ through $(\mathrm{FeS} A / B)$, and ferredoxin to NADP+ (Fig. 2).

At the same time as electrons are transported through the electron transport chain, hydrogen ions are moved from the outside and are released on the inside of the thylakoid membrane. This transfer of protons occurs by 1) actual movement of protons across the membrane through the plastoquinone pool (1.4.7) and 2) apparent movement of protons caused by the release of $\mathrm{H}^{+}$from water oxidation at the inside of the membrane, and the uptake of protons at the outside of membrane by NADP+ forming $\mathrm{NADPH}_{2}$. This movement of protons across the membrane results in the formation of a proton gradient whichis subsequently used in the formation of ATP (1.5.1). To summarise, the function of the electron transport chain is to provide energy in the form of ATP and $\mathrm{NADPH}_{2}$ which can then be used in the subsequent dark reactions to $\mathrm{fix} \mathrm{CO}_{2}$ in the form of carbohydrates.

This scheme of electron transport is generally accepted as being adequate to explain the experimental results which are available to date. However, some reports have appeared in the literature suggesting that cyclic electron transport occurs around each photo system. Arnon et al (1981) proposed a scheme in which the two photosystems operate in parallel as opposed to the series model depicted by the $z$ scheme. 


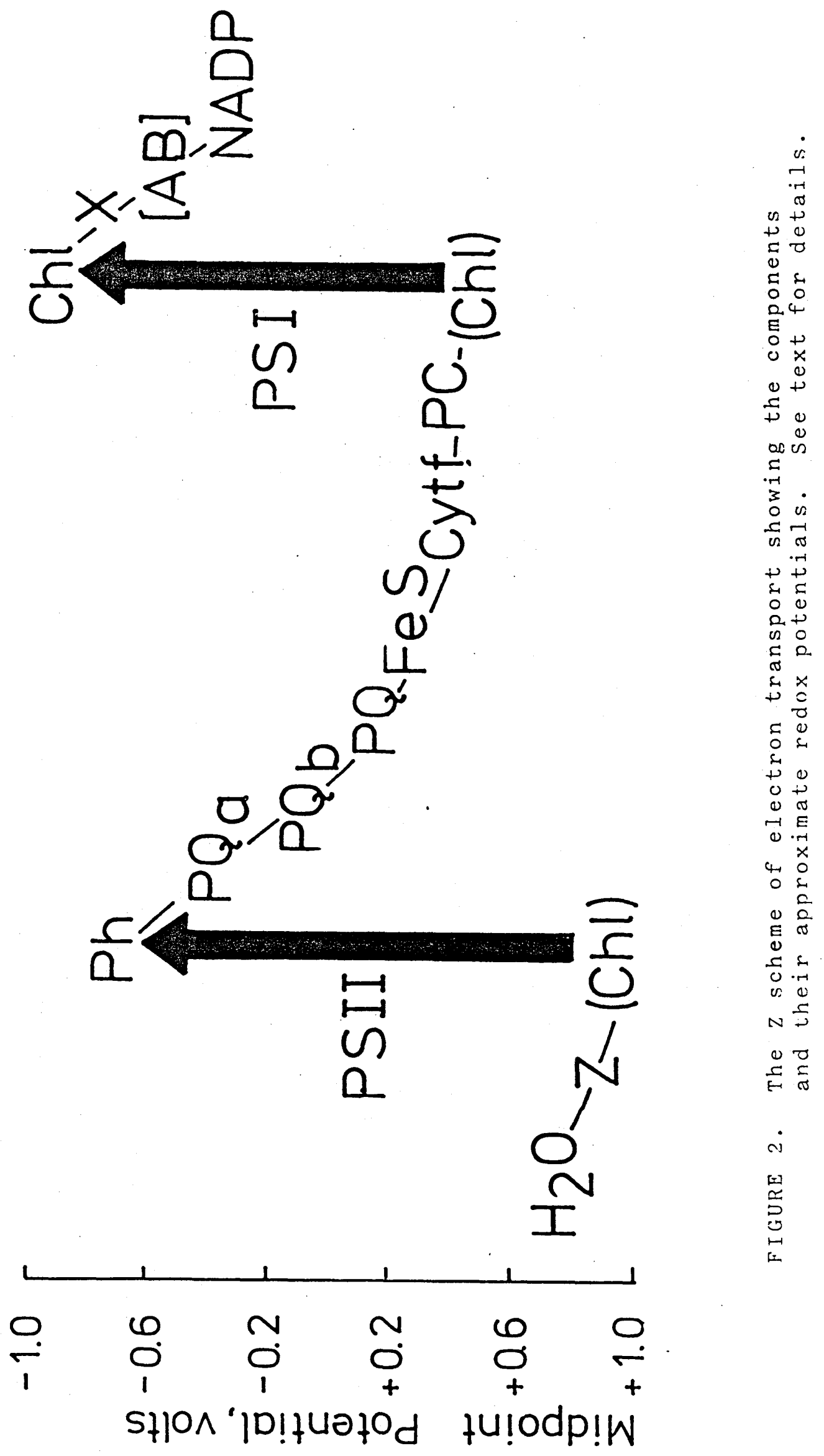


1.4.1.2 Cyclic Electron Transport.

In addition to linear electron transport (section 1.4.1.1) isolated intact chloroplasts are capable of driving cyclic electron transport: this reaction is mediated by PSI and produces ATP without reducing NADP ${ }^{+}$. Phosphorylation driven by cyclic electron transport was the first type of phosphorylation to be discovered (Arnon et al, 1954), yet the physiological significance of this is still not clear. This is due to the difficulty of measuring cyclic electron transport alone under physiological conditions because, there is no way of distinguishing between ATP produced during 1 inear from that of cyclic electron transport. Cyclic electron transport has been demonstrated in isolated intact chloroplasts but this was under non-physiological conditions: DCMU was used to inhibit linear electron transport and added dithionite poised the redox state of the components by donating electrons to the fully oxidised cyclic chain (Crowther et al, 1979).

The cyclic and non-cyclic electron transport chains have common electron carriers which are: ferredoxin; the Reiske iron-sulphur centre; plastoquinone; cytochrome $f$ and plastocyanin (Allen, 1983). On the basis of inhibitor studies with Antimycin a, cyt b 563 : has been assigned a unique role in cyclic electron transport, where it was believed to transfer electrons from ferredoxin to plastoquinone: Antimycin a was shown to inhibit the oxidation of cyt $\underline{b} 563$ and cyclic phosphorylation but it had no effect on linear electron flow (Arnon et al, 1967; Bohme and cramer, 1972). However, the site of action of Antimycin a in chloroplasts has proved to be ambiguous. 
It is not the same as in mitochondria and bacteria where it is known to inhibit electron transfer from cyt b to cyt $\underline{c}$ (slater, 1973); hence the above assignation of cyt $\underline{b} 563$ as a 1 ink between PSI and plastoquinone $\mathrm{is}$ i: tentative at best. More recently it has been suggested that the site action of Antimycin a, in chloroplasts, may be an unidentified proteinaceous component which would mediate electron transfer from ferredoxin to plastoquinone. In addition, Crowther and Hind, (1980) have proposed that an extra membrane component called $\mathrm{V}$ ", may be present in the membrane acting as a link between ferredoxin and plastoquinone.

Evidence for the involvement of plastoquinone in the cyclic chain has come from the use of DBMIB (a quinone analogue) which inhibits both cyclic and non-cyclic electron flow (Mills et al,1979; Malkin and Chain, 1980). The path of electron flow in the cyclic chain is still unclear. Although the suggestion that cyt $\underline{b} 563$ acts as a link between ferredoxin and plastoquinone is only tentative. It is also unlikely that cyt $\underline{b} 563$ connects directly with cyt $\underline{f}$ (Velthuys, 1978). Evidence from the use of the electrochromic bandshift (1.9.1.1) suggests that an extra electrogenic loop may occur when plastoquinone $\left(\mathrm{PQH}_{2}\right)$ passes one electron to cyt $\underline{f}$ which then creates the driving force for another one-electron transfer from plastosemiquinone to a b - type cytochrome (Velthuys, 1978; 1979). In order to account for the experimental results which indicate that cyt $\underline{b} 563$ may be involved in both 1 inear and cyclic electron transport two models have been proposed 1) the Q-cyle and 2) the b-cycle: these models are discussed in section 1.4.7. 


\section{4 .2 Donors to PSII.}

This part of the electron transport chain, in conjunction with the primary donor (P680) of PSII, brings about the splitting of water.

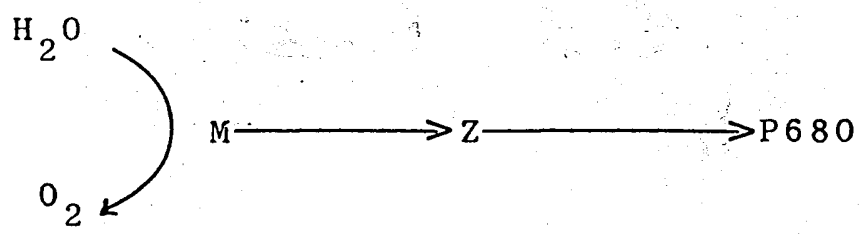

Between $\mathrm{H}_{2} \mathrm{O}$ and PSII there are two components which are shown in the scheme above: 1) $M$ is the complex which is involved in $0_{2}$ evolution; 2) $z$, the primary donor to P680. A third component which is found closely associated with PSII is cyt 1559 (Wydrinski, 1982)

The Mechanisms of $\mathrm{O}_{2}$ evolution.

oxygen is produced in the photosynthetic reaction from water oxidation:

$2 \mathrm{H}_{2} \mathrm{O} \longrightarrow 4 \mathrm{e}^{-}+4 \mathrm{H}^{+}+\mathrm{O}_{2}$

The absorption of four quanta of 1 ight is required to produce one molecule of oxygen. Each quanta is capable of forming one oxidising equivalent, therefore before oxygen can be evolved four oxidising equivalents have to be stored. Investigating the mechanism of $\mathrm{O}_{2}$ evolution Joliot et a ㄱ (1969) and Kok et a ㄱ (1970) measured the flash yield of $0_{2}$ from dark adapted algae and found a distinctive pattern of $\mathrm{O}_{2}$ evolution: no $0_{2}$ was produced on the first flash, very little on the second flash and maximum on the third. Continued repetitive flashes: : showed a periodicity of four with maximum $\mathrm{O}_{2}$ evolution occurring after the 7 th, 11 th and 15 th flashes; eventually this 
pattern was damped to give a steady state level on each flash. Following these experiments kok et a 1 (1970) proposed a charge storage model where there are 5 ' $S$ ' states which differ in the number of oxidising equivalents they store, with only $\mathrm{s}_{4}$ being able to oxidise water. The states are promoted from one level to the next by a flash of light (Fig. 3 ). oxygen is evolved after only three flashes, suggesting that one oxidising equivalent is stable in dark adapted systems. The state $\mathrm{s}_{1}$ is assumed to be stable in the dark and the $\mathrm{S}_{2}$ and $\mathrm{S}_{3}$ state deactivate to $S_{1}$ in the dark. After about 20-30 flashes a steady state is reached and no oscillations are visible; to account for this two explanations have been put forward (kok et al, 1970): 1) double hits - which would advance the system, $\mathrm{Sn} \rightarrow \mathrm{Sn}+2$ and 2) misses - which would retard the system, $\mathrm{Sn} \rightarrow \mathrm{Sn}$.

originally it was proposed that each $0_{2}$ evolving complex was uniquely associated with one PSII reaction centre and could not donate electrons to other reaction centres. Support for this proposal came from the existence of the oscillatory pattern of flash yield which was also present, albeit at a reduced level, in chloroplasts treated with DCMU (Kok et al, 1970). However, Lavorel (1978) attempted to account for 'misses' by suggesting that a mobile protein associated with oxygen evolution could store the oxidising equivalents from different reaction centres. If the mobile protein was unable to collect an oxidising equivalent this would result in the occurence of misses. 


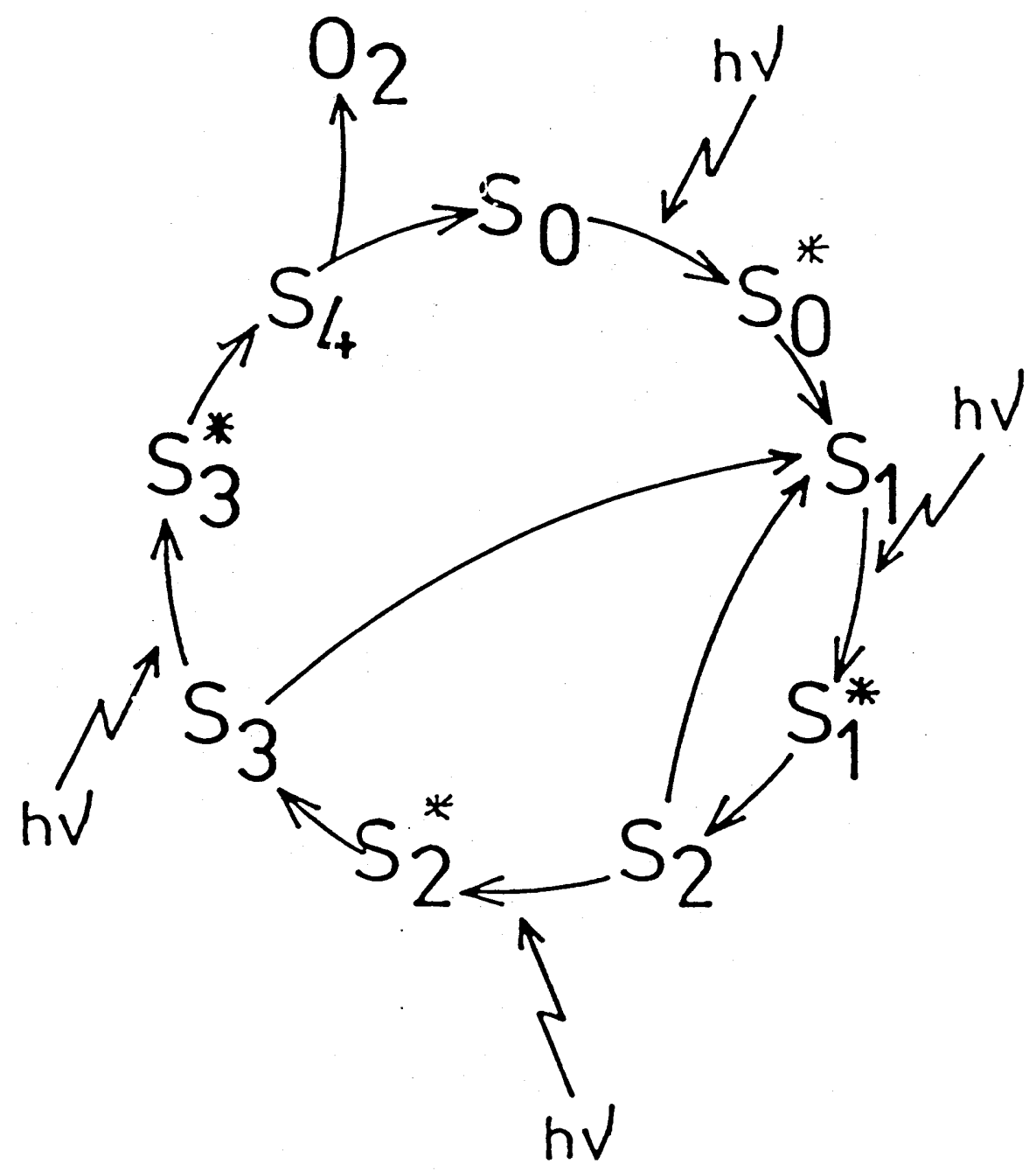

FIGURE 3 .

The 'S' state model. This shows that as light is absorbed each ' $S$ ' state is promoted from one level to the next. oxygen is evolved only after the ahsorption of 4 quanta $\left(S_{1}\right)$.

In the dark states $S_{2}$ and $S_{3}$ decay to $S_{1}$ which is stable. 
The pattern of hydrogen ion release is complex and four $\mathrm{H}^{+}$are not released in one step along with $\mathrm{O}_{2}$ evolution (Fowler, 1977) as might be expected from the ' $S$ ' state model. The most favoured pattern of $\mathrm{H}^{+}$release, as far as experimental results are concerned, is the sequence $1,0,1,2$ as the $\mathrm{S}$ states go from $\mathrm{S}_{0}$ to $\mathrm{S}_{4}$ (see Fig. 3) (Fowler,1977; Forster and Junge, 1985). Although, a pattern of 0 , $1,1,2$ was proposed by Junge and Auslander (1978).

' $M$ ' The 0xygen Evolving Complex.

The complex responsible for oxygen evolution is referred to as 'M' (also $\mathrm{Y}_{320}$, $\mathrm{S}$ or $\mathrm{L}$ ). The nature of this complex has remained elusive but there is strong evidence to suggest that it involves both manganese and chlöride, together with three polypeptides with apparent molecular weights of 16 , 23 and $33 \mathrm{KDa}$.

MANGANESE - experiments on algal nutrition indicated the importance of manganese for photosynthesis (Pirson, 1937). Later studies showed that extraction of manganese from chloroplasts using Tris or $\mathrm{NH}_{2} \mathrm{OH}$ resulted in the inhibition of oxygen evolution but not electron transport (Amesz, 1983): these experiments led to the idea that manganese acts at or near the site of oxygen evolution. Manganese can exist in a number of oxidation states and for this reason it has frequently been suggested as the charge storage device proposed in the model of kok (1970). There are three pools of manganese in chloroplasts which are referred to as weakly bound, strongly, bound, and very strongly bound. The strongly bound manganese is the pool which appears to be involved in water oxidation(Amesz,1983). When 
complexed with, for example, protein manganese gives no electron spin resonance signal (ESR) whereas free manganese gives a distinctive spectrum: therefore ESR is used to assay for free manganese (Amesz, 1983). The techniques of nuclear magnetic resonance (NMR), X-ray absorption edge spectroscopy (XAES), extended $X-r a y$ absorption fine structure (EXAFS) and lightinduced absorption changes at $320 \mathrm{~nm}\left(\Delta \mathrm{A}_{320}\right)$ have all been used in an attempt to measure the oxidation state of manganese within the membrane but as yet these approaches have not yeilded any clear cut results. It has been shown that in PSII particles a minimum of $2 \mathrm{Mn}$ per reaction centre are required for oxygen evolution but in highly active PSII particles $4 \mathrm{Mn}$ per reaction centre were required (Yocum et al , 1981). There seems to be no doubt that manganese is involved in water-splitting but the mechanism of this involvement is still unknown.

CHLORIDE - chloride was first implicated as a co-factor in oxygen evolution by Izawa et al (1969). Despite intensive study the exact role of chloride has not been identified, but there have been a number of suggestions as to its function: 1) it may be required for charge neutralisation which would in turn allow a specific conformation of the polypeptides implicated in $0_{2}$ evolution (Johnson et al , 1983);2) involvement in the stabilisation of the higher 'S ' states $\left(\mathrm{S}_{3}\right.$ and $\left.\mathrm{S}_{4}\right)$ (Itoh et al, 1984; Theg et al , 1984); and 3 ) a direct function in the water splitting reaction as suggested in the model by Sandusky and Yocum (1984).

SITEII

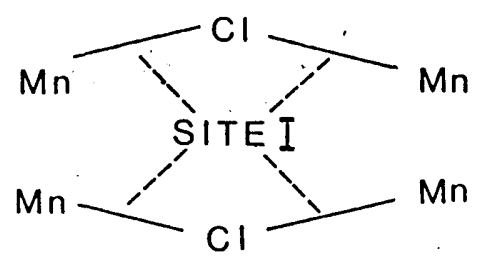


In this model a molecule of $\mathrm{H}_{2} \mathrm{O}$ is bound at site II and electrons are transferred from the site of water oxidation to $\mathrm{Z}^{+}$with the aid of the chloride bridge indicated by site I.

THE POLYPEPTIDES - three polypeptides have been suggested as having a function in oxygen evolution. When PSII particles (see 1.6.1.1) or inside out vesicles (Andersson and Akerlund, 1978) were treated with Tris, concentrated $\mathrm{NaCl}$ or subjected to high $\mathrm{pH}$, oxygen evolving activity was lost and at the same time three polypeptides, with molecular weights of 33,23 and $18 \mathrm{KDa}$, were released. This suggested that these extrinsic proteins were components of the oxygen evolving complex. Removal and reconstitution experiments have been used to investigate the individual roles of these polypeptides.

1. $33 \mathrm{KDa}$ polypeptide - removal of this polypeptide from membranes depleted of the 24 and $18 \mathrm{kDa}$ polypeptides results in an inhibition of $\mathrm{O}_{2}$ evolution (Miyao and Murata, 1984). Moreover, a $33 \mathrm{KDa}$ protein containing two bound manganese ions was isolated by Abramowicz and Dismukes (1984) and more recently using a butanol extraction procedure Yamamoto et al (1984) have also extracted a manganese containing $33 \mathrm{KDa}$ protein. on the other hand it is possible to isolate this protein containing no manganese (Miyao and Murata, 1984).

Moreover, in the absence of this protein some oxygen evolution has been observed if $\mathrm{CaCl}_{2}(150 \mathrm{mM})$ or $\mathrm{NaCl}(200 \mathrm{mM})$ are added to the membrane preparations (zimmerman and Rutherford, 1985).

2. $23 \mathrm{KDa}$ polypeptide - low levels of oxygen evolution occur in membranes which are depleted of the 23 and $17 \mathrm{KDa}$ polypeptides. Re-addition of the $23 \mathrm{KDa}$ protein restores the oxygen activity (Ljungberg et al, 1983) but later it was shown that low 
concentrations of calcium ions ( $5 \mathrm{mM}$ ) could substitute for this protein. This polypeptide also reduces the level of chloride ions required to achieve normal rates of oxygen evolution.

3. $17 \mathrm{KDa}$ polypeptide - the role of the $17 \mathrm{KDa}$ protein in oxygen evolution is unclear. It appears to be necessary for maximal rates of oxygen evolution only in thylakoids with a chloride concentration of less than $3 \mathrm{mM}$ (Miyao and Murata, 1985). This polypeptide is always released concomitantly with the $23 \mathrm{KDa}$ protein and a binding site for this polypeptide on the $23 \mathrm{kDa}$ was demonstrated (Miyao and Murata, 1983). The above data suggests that it is unlikely that these three hydrophillic polypeptides play a direct role in oxygen evolution. But they may create the right environment for this activity. In addition it is still not clear to which protein the functional manganese atoms bind - it could well be the reaction centre polypeptide of PSII ( $47 \mathrm{KDa})$ (see 1.6.1.1) to which $Z$ (see next section) is believed to be bound (zimmerman and Rutherford, 1985). To complicate the picture further a $10 \mathrm{KDa}$ protein located at the inner granal surface has recently been proposed to have a role in the mechanism of oxygen evolution (Ljungberg et al , $1984)$. 
$Z$ - the primary donor to P680 donates an electron to $\mathrm{P} 680^{\circ}$ (section 1.4 .3 ) restoring it to $\mathrm{P} 680$ and becomes'oxidised to $\mathrm{z}^{+}$, an electron passed from the $M$ complex then reduces $Z^{+}$. Electron paramagnetic resonance studies have revealed a rapidly reversible signal which appears to indicate the formation of $\mathrm{z}^{+}$. This EPR signal is called signal $I_{\text {vf }}$ and has been used in kinetic studies of this donor to PSII (Babcock and Sauer, 1973). Attempts have been made to identify this species and there is cevidence to suggest that it may be a plastoquinone-derived free radical species. (Kohl and Wood,1969:; Hales and Gupta, 1979). o'Malley and Babcock (1984) showed that various synthesised quinone radicals have similar properties as the EPR Signal II $\mathrm{I}_{\mathrm{S}} \mathrm{z}^{+}$. A binding site for $\mathrm{Z}^{+}$ on the reaction centre protein, close to the inside of the membrane, has been proposed (Babcock et al, 1983) (sections 1.6 .2 and 1.6.3.1).

Cytochrome b 559 - is present in thylakoid membranes in two different redox forms. The redox potential of one cyt $\underline{b} 559$ is $\sim+20 \mathrm{mV}$ and this is called cyt $\underline{b}$ 559 low potential the other cyt $\underline{b} 559$ has a potential of $+370 \mathrm{mV}$ and is known as cyt b 559 high potential. It has been suggested that the low potential component is a modified form of cyt b 559 H.P. (Cramer and Horton, 1975; Butler, 1978). However, fractionation of chloroplasts results in the separate isolation of these two components: cyt b 559 L.P.is loosely associated with the isolated cyt $\underline{b} / \underline{f}$ complex (under certain conditions) and cyt $\underline{b} 559$ H.P.co-fractionates with PSII. This suggests that the two forms have different and independent functions. 
As yet the function of either form of cyt $\underline{b} 559$ is still not clear although some proposals have been made. The close association of cyt $\underline{b} 55$ - HP with PSII prompted investigators to lobk for a role for this component in water oxidation. However, the photo-reduction of this cytochrome appears to be too slow for it to have a direct function in electron transport (Whitmarsh and cramer, 1977). One possibility is that it may have a safety function keeping $P 680$ in the reduced form in the event of a loss of activity of an $\mathrm{O}_{2}$ evolving centre.

There is little information available on cyt b $559 \mathrm{LP}$, although one proposal suggests that it might function as an intermediate between ferredoxin and plastoquinone in cyclic electron transport but there is no experimental evidence to support: this (Bendal1, 1982).

\section{4 .3 The PSII Reaction Centre.}

This pigment protein complex is thought to span the thylakoid membrane with the donor being situated close to the inside and the acceptor nearer to the outside of the membrane (section 1.6.2.1). The primary donor is designated P680 (Chla II). P refers to pigment and $680 \mathrm{~nm}$ is the wavelength where a photobleaching was observed (Doring et al , 1967) It has been suggested that $P 680$ is a special chlorophyll a molecule and is probably a monomer (wasielewski et al , 1981). The redox potential of $\mathrm{P} 680$ is believed to be greater than +0.8 volts which is required for the oxidation of water and although no redox potential for the oxidation of $P 680$ has been reported, it has been estimated at $\sim+1.1 \mathrm{~V}$ (Jurinsic and Govindjee, 1977). P680/P680 ${ }^{+}$is a strongly oxidising couple and because of this it is difficult to get a redox 
mediatior that would react with it without causing destruction of antennaschlorophyll (Malkin, 1982). Electrons are passed from P680 to a stable acceptor: called Qa via an intermediate acceptor pheophytin.

$\mathrm{P} 680 \longrightarrow$ Pheophytin $\longrightarrow \mathrm{Qa} \longrightarrow \mathrm{Qb}$

When Qa was held reduced an intermediate was shown to accumulate (Klimov et al, 1977) and the identity of this component is believed to be a pheophytin monomer with a redox potential of $-610 \mathrm{mV}$ which accepts electrons directly from P680 (KIimov et al, 1979). Recently chemical analysis of isolated PSII particles suggest the presence of 2 pheophytin molecules (Omata et al,1984); this is interesting considering that bacteria have 2 pheophytin molecules associated with the reaction centre where one plays an active role in photochemistry and the other has no photochemical function (okamura et al, 1982). The first stable acceptor of PSII is believed to be a quinone molecule associated with an iron atom $\left(Q^{-}-F e\right)$, according to EPR studies (Rutherford et $\underline{\text { a } 1}, 1984)$.

This component was first observed by monitoring changes in chlorophyll fluorescence intensity (Duysens and Sweers, 1963); they assigned the letter $Q$ to describe this component because when $Q$ is oxidised chlorophyll fluorescence is quenched and when $Q$ is reduced fluorescence is high. At this time the nature of this component was unknown. The redox state of the primary acceptor Qa can be measured by three methods: 1) an absorbance change at $320 \mathrm{~nm}$ which is directly related with the reduction 
of a quinone to a semi-quinone (stiehl and witt, 1968); 2) an absorbance change at $550 \mathrm{~nm}$ which results from a bandshift of the carotenoids in response to a change in the redox state of $Q$ (Knaff and Arnon, 1969) and 3 ) a change in fluorescence (Duysens and Sweers, 1963). Using the absorbance change at $550 \mathrm{~nm}$ to assess redox potential a number of results suggested an $E_{m}$ for $Q a$ of $0 \mathrm{mV}$ (Malkin, 1982). However using the fluorescence method two midpoint potentials were observed, one with an $E_{m}$ of $\sim 0 \mathrm{mV}$ and the other with an $E_{{ }^{m}}$ of $-250 \mathrm{mV}$ (Cramer and Butler, 1969; Baker and Horton, 1980). It is now believed that the primary acceptor Qa of PSII is heterogenous, consisting of $Q_{H}\left(E_{m} \curvearrowright 0 m V\right)$ and $Q_{L}\left(E_{m}-250 m V\right)$.

A secondary quinone acceptor is present and has been designated $Q b$, this component acts as a gate between the one electron acceptor $Q \mathrm{a}$ and the two electron chemistry of the plastoquinone pool. Qb accumulates two electrons before passing them on to the plastoquinone pool (Bouges-Boquet, 1973; Velthuys and Amesz, 1974). Qb is a bound oxidised quinone believed to be in exchange equilibrium with the plastoquinone pool. When Qb receives an electron from Qa it becomes strongly bound to the $32 \mathrm{KDa}$ herbicide binding protein (1.6.3.2), a second electron is then passed from Qa resulting in $\mathrm{Qb}^{-}$being reduced to $\mathrm{Qb}^{2}$. It is still unclear whether $\mathrm{Qb}^{2-}$ or $\mathrm{QbH}_{2}$ is released from the $32 \mathrm{KDa}$ protein in exchange for a quinone molecule. $\mathrm{QbH}_{2}$ may not be formed because the protons in the bulk phase are not in equilibrium with $\mathrm{Qb}^{2-}$ which is bound in a hydrophobic environment: this suggests that uptake of protons occurs at the level of the plastoquinone pool. However, Kyle (1985) proposed a model in which the species which is released from 
the protein is $\mathrm{PQH}_{2}$ (a quinol) this suggesting that $Q b$ functions as a proton transporter.

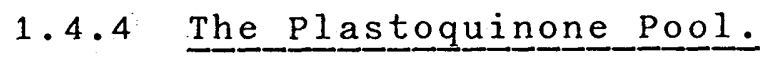

Plastoquinone is present in large amounts in the thylakoid membrane and is believed to be associated with the lipid matrix because it can be extracted from the membrane and is soluble in organic solvents and it is a hydrophobic molecule. The midpoint potential of plastoquinone is in the range of $+70-(+106) \mathrm{mV}$ (Malkin, 1982).

The function of the plastoquinone pool is twofold 1) it mediates the transfer of electrons from the acceptor side of PSII (Qb) ' to the intermediate electron carriers of the cyt $\underline{b} / \underline{f}$ complex; and 2 ) it is a hydrogen carrier and therefore has been implicated in the transport of hydrogen ions from the outside of the membrane to the inside (chemiosmotic theory see section 1.5). Many PSII centres appear to feed into a larger common pool of plastoquinone (Siggel et al, 1972) and there are 5-10 plastoquinone molecules per reaction centre (Goldbeck and Kok,1979): . Recently an additional role

for plastoquinone has been discussed : PSII and PSI are thought to be spatially separated along the membrane; PSII being mainly in the granal membranes and PSI mainly in the stromal lamellae (1.6.2.2). Plastoquinone is believed to diffuse laterally from granal to stromal regions transferring electrons between the photosystems. However the diffusion rates for plastoquinone may not be adequate to travel the distance in the time required for electron transport (Rich, 19-84). 
The separation of PSII and PSI between appressed (grana) and non-appressed membranes (stroma)

(see 1.6) and the suggested long distance diffusion of plastoquinone indicate that the electron transport chain depictedas a simple linear ( $\mathrm{z}$ scheme) scheme is over simplistic. The $Z$ scheme implies that one PSII centre and one PSI centre are statically coupled together but in this new model PSII and PSI are spatially separated, may be present in unequal stoichiometry and would be connected by a mobile quinone carrier.

The role of plastoquinone as a proton pump is discussed in the section on $Q-$ and $b$ - cycles (1.4.7) where again the picture seems to be more complex than the simpler model depicted by the $z$ scheme.

\subsubsection{Intersystem Electron_Carriers.}

\subsubsection{Reiske-Iron Centre.}

The first iron-sulphur centre of this type to be purified was extracted from mitochondria (Reiske et al, 1964); the midpoint potential of this component was between $+180-+290 \mathrm{mV}$ (Reiske, 1976). Ironsulphur centres have also been characterised in chromatophores (Evans et al, 1974; Prince et al, 1975 ) and chloroplasts (Malkin and Aparacio, 1975). The mid-point potential of this complex in chloroplasts was $+290 \mathrm{mV}$ as measured by titrating the characteristic ESR signal in the dark.

The midpoint potential of the Reiske centre and the ability of PSI light to eliminate the ESR signal of this component (Whitmarsh and Cramer, 1979) suggest that the centre lies between the plastoquinone pool and PSI in the $Z$ scheme. 
1.4.5.2 Cytochrome b 563 .

This cytochrome has previously been referred to as cyt $\underline{b} 6$ but it is probably more correct to call it cyt b 563. In freshly prepared chloroplasts more than one low potential cytochrome b can be identified: one is cyt $\underline{b} 563$ while the other is probably cyt $\underline{b}$ 559 .

The mid-point redox potential of cyt $\underline{b} 563$ is $-90 \pm 20 \mathrm{mV}$

( Crofts and wraight, 1983). Formerly this component was believed to mediate electron transfer between ferredoxin and plastoquinone in the cyclic scheme of electron transport (see 1.4.1.2). However, more recent experimental data suggest that a more complex scheme of transport occurs in this region of the chain and the involvement of cyt $\underline{b} 563$ in this is discussed in the section on $Q-$ and b-cycles $(1.4 .7)$.

\subsubsection{C.ytochrome $\underline{f}$}

cytochrome $\underline{f}$ is a $\underline{c}$ type cytochrome (Hill and Scarsbrick, 1951) and it has a mid-point potential of $+340 \mathrm{mV}$ (Malkin, 1982). Originally this component was thought to function in a straight forward manner; accepting electrons from the Reiske-iron complex and passing them onto plastocyanin and evidence from a number of sources (spectrophotometric, biochemical and genetic) supported this concept. However, there are some aspects of cyt $\underline{f}$ which are not understood. Firstly, a difference exists between the equilibriation of cyt $f$ with $P 700$ in 1 ight when compared to that in the dark (Marsho and Kok, 1970); this may relate to a change of position of the cytochrome in the membrane 
(Horton and Cramer, 1974); Secondly, the redox changes which cyt $\underline{f}$ undergoes after a single flash are not easily understood in terms of the simple linear scheme:

$\mathrm{Reiske} \longrightarrow \mathrm{cyt} \stackrel{\mathrm{f}}{=} \longrightarrow \mathrm{PC} \longrightarrow \mathrm{P} 700$

one interpretation of thesechanges is that cyt $\underline{\underline{f}}$ may pass an electron to P700 via PC oir it may pass directly to P7O0 (clayton, 1980).

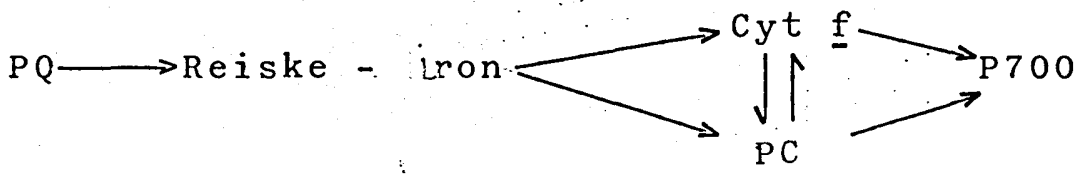

1.4.5.4 Plastocyanin

Plastocyanin is a copper containing protein (Katoh et al', 1961) having a mid-point potential of $+380 \mathrm{mV}$ (Katoh et al 1962 ). Its $\mathrm{x}$-ray structure has been determined (colman et al, 1978). The oxidation/ reduction state of plastocyanin can be measured spectro-photometrically at $597 \mathrm{~nm}$.

This component is thought to be loosely attached to the inside of the membrane where it acts as a mobile carrier of electrons between the cyt $\underline{b} / \underline{f}$ complex and the PSI reaction centre. Plastocyanin is believed to be the immediate donor to $\mathrm{P} 700$ in higher plants, but it has been shown in algae that a c type cytochrome can donate directly to PSI. By manipulating the supply of copper to microalgae the donor to PSI could be altered: either cyt $\underline{c} 553$ or plastocyanin or both were shown to supply electrons to p700 
(Sandmann et al, 1981) a scheme depicting this flow is shown in section 1.4.5.3.

Plastocyanin has the ability to store more than one electron (possibly up to four) and may thus act as a small electron pool between plastoquinone and PSI (clayton, 1980).

\subsubsection{PSI Reaction Centre.}

The function of the reaction centre of PSI is to reduce $X$ which in turn facilitates the reduction of $\mathrm{NADP}^{+}$to $\mathrm{NADPH}_{2}$. The primary.donor of PSI is referred to as P700, because it is a pigment with a major absorption band at $700 \mathrm{~nm}$; the midpoint redox potential of this component is in the range of +430 to $(+560 \mathrm{mV})$ (okamura et al, 1982 ). Until recently P700 was believed to be a dimer of Chla (Norris et al, 1974) but ESR and ENDOR studies have shown that it may be a chla monomer (Davis et $\underline{\text { a }} 1,1979$ ). A series of electron acceptors has been detected in this photosystem:

$\mathrm{P} 700 \longrightarrow \mathrm{A}_{1} \longrightarrow \mathrm{X}^{2} \stackrel{(\mathrm{A})}{\longrightarrow}(\mathrm{A}, \mathrm{B}) \stackrel{\text { Soluble }}{\longrightarrow} \mathrm{Ferredoxim} \longrightarrow \mathrm{NADP}^{+}$

The primary acceptor, $A_{1}$, is not stable nor is it well characterised but there is some evidence to suggest that it could be a Chla monomer situated close to P700 (Baltimore and Malkin, 1980a and 1980b). Measurement of the redox potential of chla in vitro gives a value of $-880 \mathrm{mV}$ and this is sufficiently low for it to act as $A_{1}$ (Fujita et al, 1978). The midpoint potential of $A_{1}$ has not been measured but it must be lower than $-730 \mathrm{mV}$, as this is the value assigned to the next acceptor ( $\mathrm{X}$ ) (Ke, 1978). 
The first stable acceptor is $X\left(\mathrm{~A}_{2}\right)$ and ESR spectra indicated that this component may be an Fe - S centre (Golbeck et al 1978) with a midpoint potential of $-730 \mathrm{mV}$. However optical changes associated with this component showed two components: 1) a broad featureless band at 400-500nm which was attributed to Fe-S absorption and 2) a number of sharp peaks at 450,670 and $680 \mathrm{~nm}$ (Shuvalov et al, 1979) which were attributed to an electric field shift in adjacent chlorphyll molecules shuvalov et al (1979) have shown that it is the same accept or which is responsible for both of these absorption changes and therefore the identity of $\mathrm{X}$ is still uncertain. It may either be a modified $\mathrm{Fe}-\mathrm{S}$ centre or an organic free radical $-\mathrm{Fe}^{2+}$ complex in analogy with PSII $(Q-F e)(1.4 .2)$.

The secondary acceptors denoted $\dot{A}$ and $B$ are bound Fe-S centres, as indicated by ESR spectra (Malkin and Bearden, 1971), having redox potentials of $-530 \mathrm{mV}(\mathrm{A})$ and $-580 \mathrm{mV}(\mathrm{B})$. The way in which electrons pass through these centres is unclear: it is not known if either $A$ or $B$ accept directly from $X$ or if both can (okamura et al,1982). Electrons are passed from $X$ to the (AB) centre then to ferredoxin from where. there are two separate pathways: 1) reduction of NADP ${ }^{+}$ mediated by ferredoxin - NADP reductase or 2) into the cyclic pathway (Bohme and Cramer, 1972) (see section $1.4 .1 .2)$.

Ferredoxin functions as a one electron carrier having a midpoint potential of $-420 \mathrm{mV}$ (Tagawa and Arnon, 1962). Ferredoxin - NADP reductase is a flavoprotein and contains one mole of FAD per, $40 \mathrm{KDa}$ of protein, the midpoint potential of this component has been reported to be $-360 \mathrm{mV}(\mathrm{pH} 7.0)$ (Kierns and Wang, 1972). In 
the presence of NADPH two one-electron steps appear to occur in the reduction reaction as was shown by measuring the midpoint redox potentials.

\section{4 .7 Q- and $b$ - Cycles of Electron Transport.}

The Q-cycle (Mitchell, 1976) and the b-cycle (wikstrom, 1973) are two alternative schemes of electron transport which were proposed to describe electron and proton transport through the cyt $\underline{b} / \underline{f}$ complex (1.6.3.3). Although experimental evidence suggests that a simple linear pathway is inadequate, these models are only working hypotheses. Support for a Q- or b-cycle model is: a) the stoichiometry of proton to electron transfer through the $\underline{b} / \underline{f}$ complex is expected to be $1: 1$, but measurements of $2: 1$ have been made, albeit under reducing conditions (i.e. where the Reiske-iron complex and cyt $f$ are reduced) (Fowler and Kok, 1976); b) the observation that cyt $\underline{b} 563$ can be reduced by ferricyanide whilst cyt $f$ is oxidised This was seen in isolated cyt b/f complexes from chloroplasts and demonstrated the phenomenom of oxidant-induced reduction similar to that seen in mitochondria and bacteria (Hauska et $\underline{a} 1,1983$ )

(n... ; c) the slow rise phase (phase b) of the electrochomic band-shift is believed to indicate an extra electrogenic step in the membrane (see section 1.9.1.1 for details). In a Q-cycle the onset of phase $b$ is associated with the outward transfer of an electron which is followed by transfer of an extra proton, whilst in b-cycle the extra electrogenic step would be associated with the transfer of an extra proton across the membrane. 
Electron Flow through the $\underline{b} / \underline{f}$ complex - both the Q- and b-cycles predict that the two electrons entering the $\underline{b} / \underline{f}$ complex come from the plastoquinone pool through a quinol molecule. A concerted reaction takes place in which the quinol reduces first the Reiske-iron centre then cyt $\underline{b}$ 563. The Reiske iron centre is then oxidised by cyt $\underline{f}$ and cyt $\underline{b}$ is oxidised by a quinone ( $Q-c y c l e)$ or semiquinone $\underline{b}-$ cycle (Fig.4): in both cases quinol is regenerated. The difference between these two models is that in the Qcycle proton transfer across the membrane is brought about by plastoquinone while in the b-cycle cyt $b$ is responsible for proton transfer. In both of these models no direct connection occurs between cyt $\underline{b} 563$ and cyt $\underline{f}$; evidence supporting this came from studies carried out under conditions where cyt $\underline{f}$ was held oxidised and cyt b re-oxidation was slowed down rather than accelerated (Velthuys, 1979). Velthuys (1979) proposed a modified Q- cycle where a second concerted reaction by another plastoquinol molecule leads to the reduction of a second cyt $\underline{b} 563$ molecule; the two reduced cytochromes b co-operate to reduce a plastoquinone molecule close to the outside of the membrane. Evidence supporting this type of modified Q- cycle has come from Joliot and Joliot (1984). Using flash excitation under oxidising conditions they studied electron transfer between the two photosystems and concluded that under these conditions a Q- type cycle was operating. At present the available evidence seems to favour a Q-cycle rather than a b-cycle. 

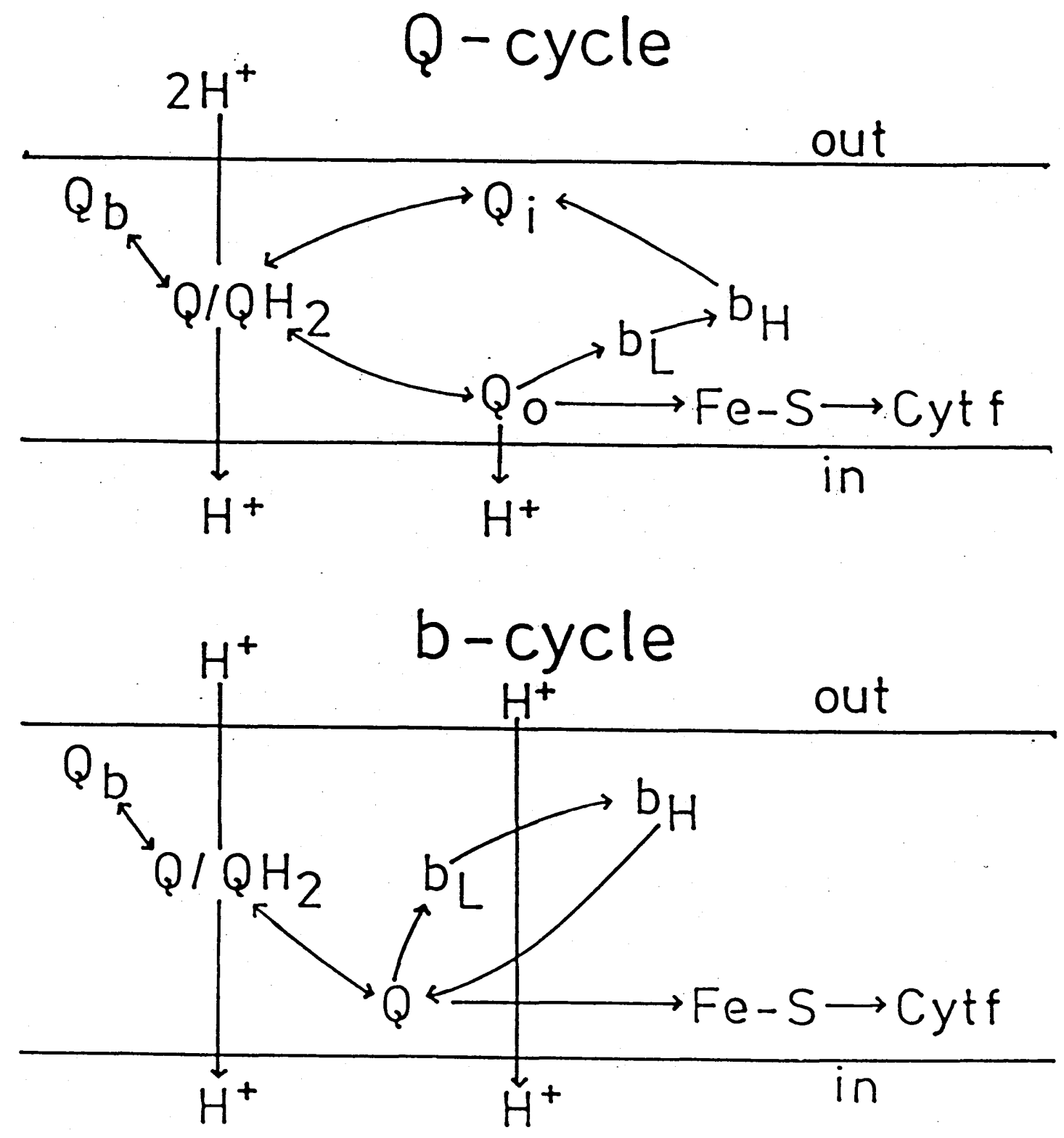

FIGURE 4 .

This figure depicts the Q- and b-cycle models of electron transport. In both models quinol reduces the Reiske ironsulphur centre then cyt $f$ whilst another electron goes to cyt b. In the Q-cycle the initial state is $\bar{r}$ egenerated when cyt $f$ re-oxidises the Reiske,centre and quinone re-oxidises cyt $b$ with removal of a protcn from outside the membrane. In the bcycle after oxidation of the Reiske-centre-by cyt $f$ a second reaction with another quinol re-reduces the reiske centre but the electron on cyt b regenerates quinol from quinone. Redrawn from Crofts and wraight (1983) 
The role of a Q--or b-cycle in steady state electron transport is still in some doubt: it is believed by some that these cycles only operate in cyclic electron transport (Benda11, 1982). However, it has been shown that an extra proton is translocated and phase b of

$\Delta A_{515}$ is evident during non-cyclic transport (Velthuys, 1980). Other groups believe that the Q-. or b-cycle operates under the regulation of the plastoquinone pool (Rathenow and Rumberg, 1980) in conditions where electron flow is sub-optimal. An alternative view has been suggested by 01sen et al (1980), who proposed that no extra proton translocation occurs in either a Q- or b-cycle and that the experimental results can be explained by the action of a semiquinone dismutase reaction: this is the reduction of a molecule of semiquinone to the quinol by another molecule of semi-quinone which is itself oxidised to a quinone. Evidence supporting the $Q$ type model came from experiments on isolated cyt b/f complexes which had been incorporated into phospholipid vesicles in this system proton translocation was demonstrated and in addition oxidant-induced reduction of cyt b 563 was observed under oxidising conditions (Hauska et $\underline{\text { al }}$, 1983 ).

Whilst the evidence seems to favour the existence of a Q-cycle experimental anomalies remain in the chloroplast system. Therefore even although conclusive evidence has been shown for the existence of a Q-cycle in mitochondria and bacteria (Slater, 1983) more evidence is required before it can be assumed that this cycle is operating in chloroplasts. 


\subsection{Photophosphorylation.}

Photophosphorylation is the light-dependent formation of adenosine triphosphate (ATP) from adenosine diphosphate (ADP) and inorganic phosphate. It was first observed in isolated chloroplasts by Arnon et al $(1954)$. The energy required to form ATP is produced in the electron transport reactions and a number of hypotheses have been put forward to explain the mechanism which couples electron transport to phosphorylation: a) the conformation hypothesis (Boyer, 1965; slater, 1974); b) the chemical hypothesis (slater, 1953); c) the chemiosmotic theory (Mitchell, $1961)$.

The conformation hypothesis predicts that electron transport generates an "energy-rich" protein conformation but this theory has not been able to account for the existing experimental data. The chemical hypothesis falls down on three counts: 1) no chemical intermediate has been identified; 2 ) intact vesicles are required for phosphorylation and the chemical hypothesis has no role for vesicles and 3 ) the chemical diversity of 'uncouplers' which inhibit phosphorylation. Many of the experimental findings can be explained within the framework of the chemiosmotic theory and it is this hypothesis that is widely accepted as the mechanism of phosphorylation. According to the chemiosmotic theory an electrochemical potential gradient of hydrogen ions provides the energy required to drive ATP synthesis. This proton gradient is produced during light-driven electron transport when hydrogen ions are transferred into the intrathylakoid space at two sites: 1) on the donor side of PSII from the splitting of water; 
2) through the plastoquinone pool, which takes up

a proton and transfers it across the membrane (see Fig. 5).

\subsubsection{The Chemiosmotic Theory.}

Mitchell (1961) postulated that there were certain pre-requisites for phosphorylation: 1) the electron transport carriers are vectorially arranged across the membrane and that some components can transfer hydrogen ions as well as electrons. This arrangement results in the creation of an electric field( $\Delta \dot{\Psi})$ across the membrane field and in a transmembrane proton gradient $(\Delta \mathrm{pH}) ; 2)$ the ATPase enzyme complex is anisotropically arranged in the membrane and couples ATP synthesis to the movement of protons: protons pass through the membrane, via this complex, driven by an electrochemical potential difference.conversely hydrolysis of ATP will be capable of driving hydrogen ions through the ATPase complex into the intrathylakoid space; 3 ) the phosphorylation reactions are dependent on the presence of a closed vesicle which has a low conductivity to protons.

This theory lent itself to experimental investigation and a large amount of data now exists in support of this hypothesis.

1. The Proton Gradient.

Light-induced proton uptake by isolated chloroplasts was demonstrated by Jagendorf and Hind (1963). In addition it was shown that by transferring chloroplasts from $\mathrm{pH} 4$ to $\mathrm{pH} 8$ in the dark results in the formation of ATP (Hind and Jagendorf, 1965). 


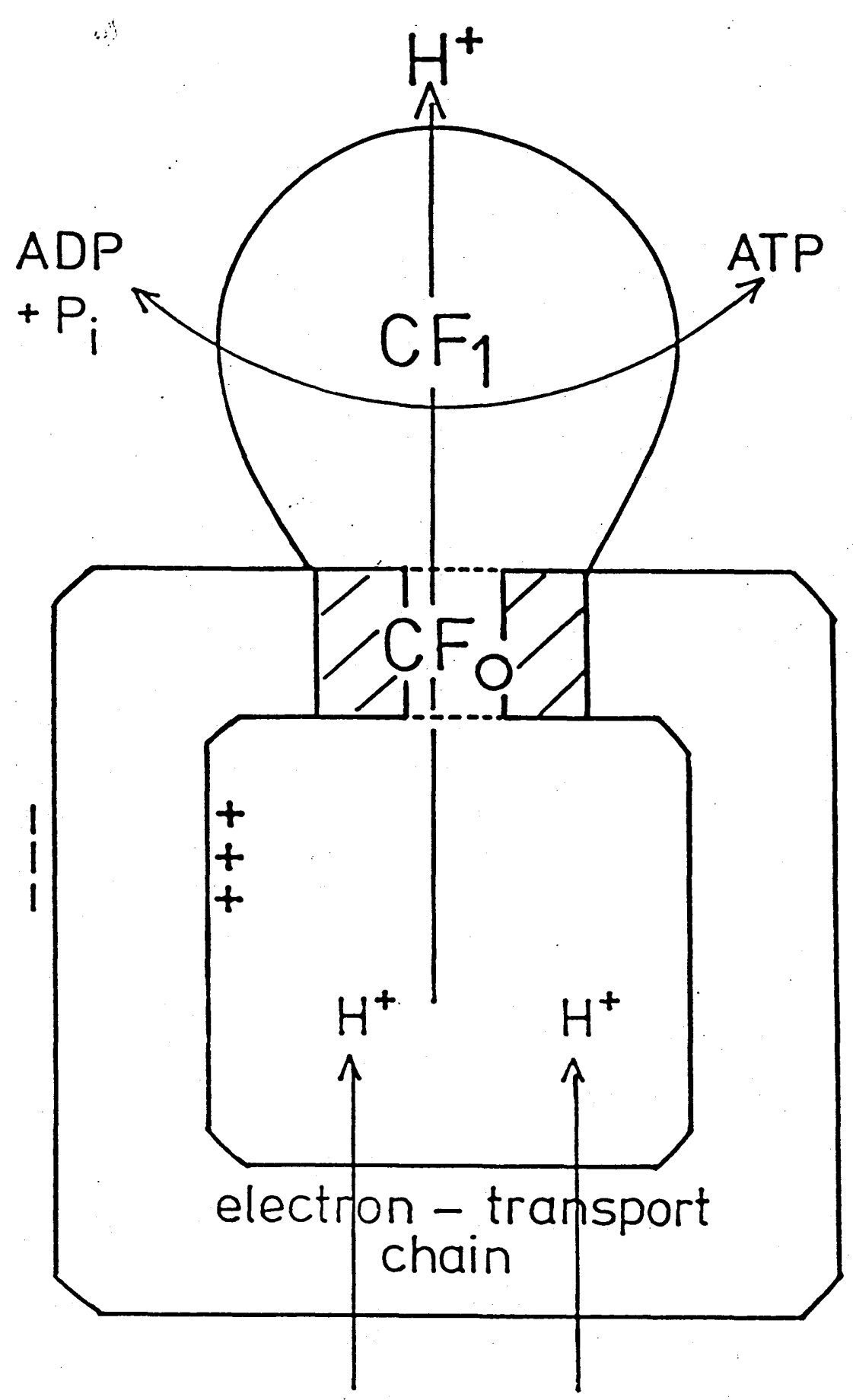

FIGURE 5.

In the chemiosmotic theory the electron transport reactions result in the formation of an electrochemical gradient of protons. This gradient is a store of potential energy and when it is dissipitated via the ATPase complex $\left(C_{0}-C F_{1}\right)$ ATP is formed from ADP and $P_{1}$. 
Proton uptake induced by ATP hydrolysis in thylakoids in the dark has also been demonstrated (Carme1i, 1970).

2. The ATPase Complex.

The ATPase complex has been shown to span the membrane: $\mathrm{CF}_{0}$ is the membrane spanning region; $\mathrm{CF}_{1}$ is the extrinsic catalytic portion (see 1.6.3.4). Removal of $\mathrm{CF}_{1}$ from the membrane results in the inhibition of proton uptake and re-addition restores the function (McCarty, 1971). Moreover removal of $\mathrm{CF}_{1}$, results in an increase in the conductivity of the membrane and either re-addition of $\mathrm{CF}_{1}$ or use of Dicyclohexylcarbodiimide (DCCD) can restore. low conductivity (McCarty and Racker, 1967).

\section{Membrane Integrity.}

Addition of ionophores, detergents or other materials which cause membrane disruption result in an inhibition of ATP synthesis (Nicholls, 1982).

\subsubsection{Energetics of ATP Synthesis.}

The electrochemical potential gradient of protons formed during electron transport can be described thermodynamically as:

$$
\Delta \mu_{\mathrm{H}^{+}}=\mathrm{F} \Delta \Psi-2.303 \mathrm{RT} \Delta \mathrm{pH}
$$

where $\Delta \mu_{H^{+}}$is the difference in electrochemical potential between protons on the inside of the membrane and those on the outside; $F$ is the Faraday;

$\Delta \boldsymbol{\Psi}$ is the electric potential difference across the membrane; $\Delta \mathrm{pH}$ is the difference in proton concentration across the membrane. $\Delta \mu_{H^{+}}$is the maximum Gibbs free energy available from the electrochemical potential gradient and has the units $\mathrm{K} \mathrm{Cal} \mathrm{mole} \mathrm{C}^{-1}$. 
It is possible to define the electrochemical potential gradient in terms of electrical units where

$$
\Delta \mu_{\mathrm{H}^{+}} / \mathrm{F}=\Delta \mathrm{p}=\Delta \Psi-2.303(\mathrm{RT} / \mathrm{F}) \Delta \mathrm{pH}
$$

which can be expressed as:

$$
\Delta \mathrm{p}=\Delta \Psi-59 \Delta \mathrm{pH}
$$

Mitchell referred to $\Delta p$ as the proton motive force (PMF), which has units of $\mathrm{mV}$.

It can be seen from the above equation that either $\Delta \boldsymbol{\Psi}$ alone or $\boldsymbol{\Delta} \mathrm{pH}$ alone can drive ATP synthesis, as found experimentally by Jagendorf and Uribe (1966) and Graber et al (1977). The two separate components of $\Delta p$ are formed at different times after illumination of chloroplasts. At first lover the first few seconds) ATP synthesis is driven purely by $\Delta \boldsymbol{\Psi}$ subsequently, under steady state conditions, only

$\Delta \mathrm{pH}$ is detectable (Ort and Melandri, 1982).

\subsubsection{Stoichiometry of $\mathrm{H}^{+} / \mathrm{ATP}$.}

It is now well established that the proton gradient $(\Delta p)$ is capable of driving ATP synthesis and that the ATPase enzyme functions as a proton translocator. What is not known, with any degree of certainty, is the number of hydrogen ions which are required to pass through the complex in order to form one molecule of ATP. Values obtained range from 2 to $4 \mathrm{H}^{+} / \mathrm{ATP}$ but there are problems associated with the methods used to make the measurements (Ort and Melandri, 1982). Estimates of $\quad \Delta G_{\text {ATP }}^{\prime}$ for ATP synthesis suggest that thermodynamically a ratio of at leas $t 3 \mathrm{H}^{+} / \mathrm{ATP}$ is required. $\Delta G_{A T P}^{\prime}$ or ATP synthesis:

$$
\begin{array}{cl} 
& \Delta G_{A T P}^{\prime}=\Delta G_{A T P}^{O \prime}+R T \text { In } \frac{[A T P]}{[A D P]\left[P_{i}\right]} \\
\text { where } & \Delta G_{A T P}^{O \prime}=. \quad R T \text { In } K_{\text {eq }}
\end{array}
$$




$$
\Delta G_{A T P}^{\prime}=8.7+1.36 \log \frac{[A T P]}{[A D P] \cdot\left[\mathrm{P}_{i}\right]} \quad \mathrm{KCal} \mathrm{mol}^{-1}
$$

Taking the worst case where the ATP concentration is high and $A \supset P$ and $P_{i}$ concentrations are low, 14.1:KCal ATP mole -1 would be required.

It is possible to express Gibbs free energy change for ATP synthesis in terms of $\Delta \mathrm{pH}$ and $\Delta \Psi$ :

$$
\Delta \mu_{H^{+}}=1.36(\Delta \mathrm{pH})+(\Delta \Psi / 59)
$$

Taking $\Delta \mu_{\mu^{+}}$for ATP synthesis equal to $14.1 \mathrm{~K}$

Cal mole $\mathrm{e}^{-1}$ and if $\mathrm{H}^{+} / \mathrm{ATP}$ is equal to 2 then $\Delta \Psi$ equals $342 \mathrm{mV}$ and $\mathrm{pH} 5$ units, these are values higher than that found. However, if $\mathrm{H}^{+} / \mathrm{ATP}$ is equal to 3 then $\Delta \Psi$ becomes $208 \mathrm{mV}$ and $\Delta \mathrm{pH}=3.3$ units and these two values correspond more closely to measurements made in chloroplasts.

\subsubsection{The ATPase Enzyme Complex.}

The mechanism of coupling of electron transport to ATP synthesis is fairly well established. Most research in recent years has concentrated on three factors involving the ATPase complex: 1) its structure (see 1.6.3.4); 2) the functional properties of the complex; and 3 ) the molecular mechanism by which the enzyme forms ATP from ADP and phosphate ( $\left.P_{i}\right)$.

1.5.2.1 Functional properties of the enzyme.

The ATPase complex has two catalytic forms: 1) as an ATP synthetase; 2) as an ATP hydrolase. The reversible reaction catalysed by this enzyme is

$$
\mathrm{HH}^{+}+\mathrm{ADP}+\mathrm{P}_{\mathrm{i}} \longrightarrow \mathrm{HH}^{+}+\mathrm{ATP}+\mathrm{H}_{2} \mathrm{O}
$$


The phosphorylation and hydrolyis activities of the ATPase enzyme complex are under strict regulatory control. It has been shown that, in isolated thylakoid membranes, activation of this complex is required in order to observe any catalytic activity (Harris and Crofts,1978; Mills and Mitchell,1982). Activation of the complex is believed to be mediated by an electrochemical gradient of protons $\left(\Delta \mathrm{H}^{+}\right)$which is generated by light-induced electron transport. It has been proposed that this complex undergoes conformational changes in the light which result in the release of tightly bound nucleotidesit is thought that this is part of the activation process (McCarty and Carmeli,1982 for review).

In addition to the $\Delta \mu \mathrm{H}^{+}$- regulated activation process there is a second type of regulation. This is called thiol modulation and is controlled by the redox state of certain thiol/disulphide groups in CF 1. In isolated thylakoid membranes hydrolysis of ATP is only unmasked when, on top of the $\Delta \mu^{+}$required for activation, a pretreatment is given in the presence of a thiol compound (Petrack et al, 1965). The hydrolysis activity thus produced is depenent on the presence of $\mathrm{Mg}^{2+}$ and was stable in the dark for several minutes. The proteolytic enzyme trypsin can also induce this activity (Lynn and straub, 1969): comparing the effects of dithiothreitol(DTT) with trypsin suggested that they acted in a similar manner but that trypsin induced a degree of uncoupling. Neither DTT or: trypsin stimulate hydrolysis if thylakoids are pretreated in the dark and it has been proposed that the conformational change induced on light activation reveals a sensitve site on $\mathrm{CF}^{\prime} 1$. Weiss and MCCarty(1977) showed that a sulphydryl group on tha $\gamma$ sub-unit of CF 1 was exposed on membrane energisation and it is thought that DTT and trypsin may modulate the enzyme by altering the state of that bond.

These two levels of regulation $\left(\Delta \mu \mathrm{H}^{+}\right.$and redox) were observed in vitro but more recently a similar in vivo 
ATPase regulatory system has been identified by Mills and Hind(1979). They showed that intenseillumination of isolated intact chioroplasts"results in the stimulationof ATP hydrolysis, whilst in lysed chloroplasts this effect was lost. Subsequently it was shown that stromal proteins, possibly thioredoxin, could mimic the effect of DTT on stimulating the ATPase activity (Milis et al, 1980). Moreover, the thiol modulated chlorplasts required a lower $\Delta \mathrm{HH}^{+}$threshold for; ATP synthesis (Mills and Mitchell,1982). In addition it has been shown that the initial rate of phosphorylation was stimulated in thiol modulated thylakoids. From this and other data it has been proposed that thiol modulated ATPase becomes active over a lower $\Delta \mathrm{pH}$ range and thereby reduces the kinetic limitation on ATP synthasis imposed by the activation process(Mills and Mitchell,1984).

1:5.2.2 Nucleotide Binding.

The interactions of $\mathrm{CF}_{1}$ with adenine nucleotides has been intensively studied in an attempt to elucidate the molecular mechanism of ATP synthesis.

Three distinct nucleotide binding sites on $\mathrm{CF}_{1}$ have been found: 1) site one contains tightly bound ADP which undergoes only slow exchange with ADP in the medium (Carlier and Hammes, 1978), or with ATP (shoshan et al, 1978). In order to remove the ADP from this site it is necessary partially to denature the enzyme; 2) site two binds ATP and requires the presence of magnesium (Shoshan et a, 1978). The properties of this site are unchanged upon activation of the ATPase; 3 ) site three binds a number of different nucleotides including ATP, ADP and GTP. This site exchanges nucleotides in the light. 
None of these sites has so far been assigned a direct catalytic function (McCarty and Carmeli, 1982). On illumination of thylakoids, nucleotides bound to $C F_{1}$ undergo a rapid exchange with $A D P$ in the medium (Harris and slater, 1975) and this exchange is sensitive to uncouplers. In addition the amount of $A D P$ bound to $C_{1}$ in the light is substantially less than: that bound in the dark (strotmann et a 1 , 1979). This exchange site does not appear to be directly involved in catalysis but it may be involved in $\mathrm{Mg}^{2+}$ - ATPase activity.

\subsubsection{Molecular Mechanisms.}

The details of the mechanism of ATPase synthesis are still unknown although a number of hypotheses has been suggested.

1) Mitchell (1974) proposed that protons were directly involved in the catalytic function: he suggested protonation of phosphate occurs inducing the formation of a positively charged phosphorus centre. Nucleophillic attack by the $A D P-0^{-}$on phosphate displaces $\mathrm{OH}_{2}^{+}$forming-ATP.

2) Boyer (1977) suggested an indirect role for protons; the proton gradient causing a conformational change in the enzyme inducing ATP synthesis.

3) Racker (1977) proposed that $\mathrm{Mg}^{2} \pm$ induced conformational changes cause a formation of a phosphoryl enzyme intermediate leading to ATP synthesis. This was an analogy of the $\mathrm{Ca}^{2+}$-ATPase of the sacroplasmic reticulum.

4) Gresser et al (1982) presented a simple interacting three-site mechanism. This model predicts that each site is identical and goes through three major stages: for ATP hydrolysis- ATP binding, interconversion to $\mathrm{ADP}+\mathrm{Pi}$ and then release of $\mathrm{ADP}+\mathrm{Pj}$. In addition it was proposed that there is co-operativity between the catalytic sites, this resulting in acceleration of the release of the products from one site when substrate is bound at another site. 
In a recent review (Mitchell, 1985) the molecular mechanism of ATP synthesis is discussed and a new hypothesis for this mechanism is suggested: the rolling well and turnstile mechanism. In this model it is proposed that the $\alpha, \beta$ and $\gamma$ subunits (see $1.6 .3 .4)$ rotate into specific positions in relation to each other. The protons injected into the protongate of the $Y$ subunit, by the proton motive force ( $i .5 .1)$ are directly involved in this mechanism.

\subsection{THE THYLAKOID MEMBRANE.}

The preceding sections in this introduction have described, in detail, the reactions which take place on the thylakoid membrane. This section will deal with the structural importance of these membranes in the control of these functions and the relationships between the structural entities and the functional components.

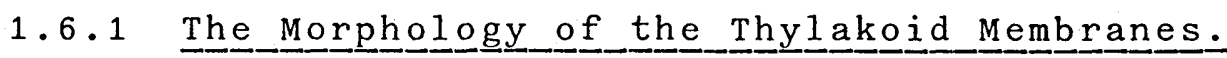

Electron micrographs of chloroplasts show two structural groups of thylakoid membranes: 1) granal stacks where thylakoid membranes are closely appressed and 2) stromal membranes which are non-appressed (see 1.2“Fig. 1). When isolated chloroplasts are osmotically shocked intact thylakoids are obtained, but in order to maintain granal stacks in these membranes it is necessary to include divalent cations $\geqslant 2 \mathrm{mM}$ in the bathing medium (Izawa and Good, 1966). The requirement for cations is non-specific in that any species of divalent cation at a concentration of

$\geqslant 2 \mathrm{mM}$ will maintain granal stacks. Moreover trivalent cations at very low concentrations and 
monovalent cations at higher concentrations ( $>100 \mathrm{mM})$ can also maintain granal stacks. These observations led to the proposal that cations allow membrane appression by electrostatic screening of the negative charges on the membrane (Barber, 1980). In addition, Mullet et al (1981) proposed that a positively charged $2 \mathrm{KDa}$ portion of the LHCP (Light harvesting chlorophyll protein) associated with PSII, is also responsible for stabilising the association between appressed membranes. This proposal received support from Jennings et $\underline{\text { a }] \text {. }}$ (1978) who showed that on removal of this $2 \mathrm{kDa}$ fragment using trypsin, cations were unable to induce restacking of grana.

\subsubsection{Regulation of Energy Redistribution.}

The distribution of absorbed energy between PSII and PSI in isolated thylakoid membranes can be manipulated by changing the cation concentration of the bathing medium (Barber, 1976). When suspended in a low-salt medium, thylakoids unstack and show a preferential distribution of energy in favour of PSI at the expense of PSII. Re-addition of cations causes granal stacking and a shift in energy distribution in favour of PSII. But in vivo complete destacking does not occur and is therefore not thought to be directly involved in the regulation of energy distribution.

Energy redistribution in vivivo has been shown to be under the control of a membrane bound kinase and phosphatase (Bennet and Allen, 1981): phosphorylation of a surface-exposed segment. of LHCPII causes an increase in energy distribution from PSII to PSI. This enzyme reaction is dependent on the redox state of the plastoquinone pool (Horton and Black, 1980; Allen et al, 1981). If the pool is reduced 
phosphorylation occurs and energy is transferred to PSI, but when the pool is oxidised dephosphorylation occurs and energy is diverted to PSII. This does not explain the actual mechanism which brings about the distribution of energy in vivo. However, Barber, (1982) has suggested that phosphorylation of the LHCP will alter the overall electrical charge of the protein: this would cause repulsion between phosphorylated components allowing a small degree of unstacking, and this would lead to the migration of PSII - light harvesting complex II (LHCII) into non-appressed regions where energy transfer between the photosystems is favoured. Recently Briantais et al (1984) gave further evidence supporting this hypothesis showing that lateral segregation of membrane components is important in controlling energy transier between photosystems.

\subsubsection{The Molecular structure of the Thylakoid Membrane.}

The thylakoid membrane is similar in structure to most biological membranes and is composed of a lipid bilayer which is interspersed with both extrinsic and intrinsic proteins, some of these spanning the membrane (Singer and Nicolson, 1972). There are about 30-50 thylakoid polypeptides, a large number of which are arranged within four large, membrane spanning, protein complexes, (Anderson and Andersson, 1982). Three of these protein complexes are involved in electron transport: PSII-LHCII, PSI-LHCI and the cytochrome $\underline{b} / \underline{f}$ complex (cyt $\underline{b} / \underline{f})$. Additionally there is the ATP enzyme complex which has a dual function: ATP synthesis and ATP hydrolysis (Fig 6).

The lipids in the thylakoid membrane are unusual in that they 
are mainly uncharged polar galacto-1ipids whilst most other biological membranes are usually composed of phospholipids. The galacto-lipids in the thylakoid membrane are monogalactosyldiacyl glycerol (MGDG) and digalactosyldiacylglycerol (DGDG) with a minor contribution by the acidic lipids sulphoquinovosyldiacyl glycerol (SQDG) and phosphatidylglycerol (PG). All of these lipids are highly unsaturated and this means that the bilayer of the membrane is fluid. The function of these lipids is presumably to help maintain the structural organisation of proteins in the membrane and to provide a fluid matrix for diffusional processes to occur (Barber, 1984).

\subsubsection{Transverse Asymmetry.}

It has been believed for some time (Trebst, 1974) that, in keeping with the postulates of the chemiosmotic theory (1.5.1), these protein complexes are vectorially arranged across the thylakoid membrane. The donors in both reaction centres are nearer to the inside of the membrane and the acceptors are nearer to the outside (Fig. 6). More recent evidence supporting this arrangement has come from the use of inside-out thylakoid vesicles (Akerlund et al, 1976). In particular the use of these vesicles has shown that the 33,23 and $16 \mathrm{kDa}$ proteins implicated in $\mathrm{O}_{2}$ evolution (Andersion et al,1981) and plastocyanin the donor to P700 (Anderson and Andersson, 1982) are located on the inside of the thylakoid membrane. It has also been demonstrated that a number of polypeptides span the membrane: the proteins of the Cyt $\underline{b} / \underline{f}$ complex (see 1.6.3.3); the light harvesting pigments of PSII (Andersson et al, 1981); and the reaction centre polypeptides which are $68 \mathrm{kDa}$ protein of PSI and the 41 and $47 \mathrm{KDa}$ proteins of PSII (Anderson, 1984). 


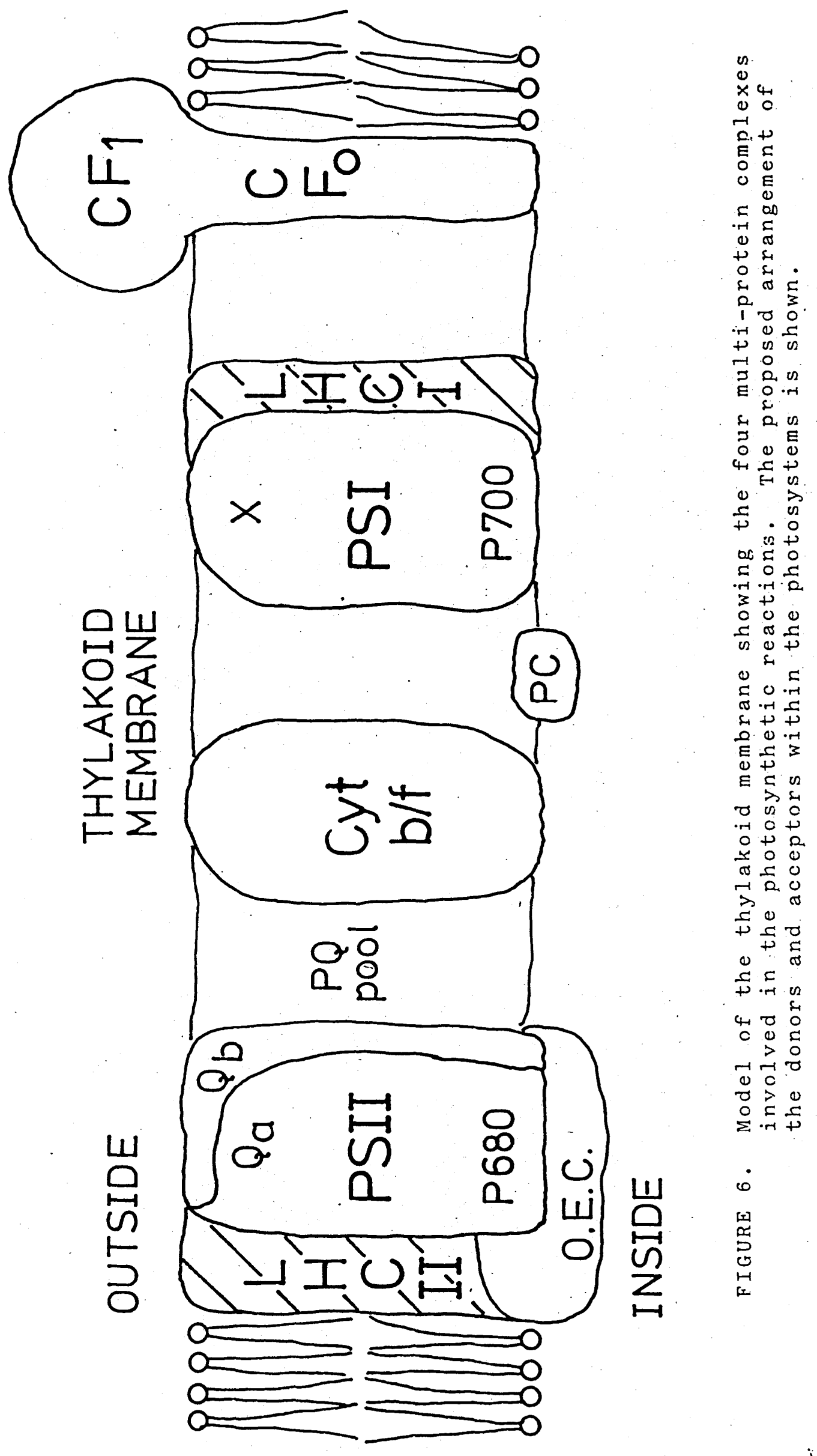




\subsubsection{Lateral Asymmetry.}

In recent years it has been suggested that, in addition to transverse asymmetry, the membrane protein complexes also display lateral asymmetry between appressed and non-appressed regions of the membrane (Anderson, 1984; Barber, 1984).

The first evidence to suggest an asymmetrical distribution of the protein complexes along the membrane came from early fractionation studies (Anderson, 1975); PSI was shown to be located mainly in non-appressed membranes and PSII was enriched in appressed membranes. In addition freeze-fracture electron microscopy revealed that distinct differences in the size and number of particles existed between stacked and unstacked regions (Arntzen, 1978). Mcre recently inside out thylahoid vesicles have been prepared and these membranes, which derive from appressed regions, are enriched in PSII activity (Andersson et al , 1981). The ATP enzyme complex and ferredoxin NADP-reductase are found only in the peripheral regions of appressed membranes and in the non-appressed regions (Miller and staehlin, 1976) The distribution of the Cyt $\underline{b} / \underline{f}$ complex is still in dispute since some authors suggest that it is uniformly distributed along the membrane (Cox and Anderson 1981; Morschel and Staeh1in, 1983). on the other hand there is some evidence to suggest that it is only present in the non-appressed membranes: highly enriched PSII particles derived from appressed lamellae contained no cyt b/f complex (whitford et $\underline{\text { al }}$, 1983). Barber (1984) proposes that this lateral heterogeneity is brought about by the different surface 
properties of the protein complexes, and in keeping with this concept hesuggests that the cyt $\underline{b} / \underline{f}$ complex would be located mainly in the non-appressed membranes and in the margins of the appressed membranes.

Based on experimental results it was concluded that PSII is located mainly in the region of appressed membranes and that PSI is located in the nonappressed membranes and granal margins (Anderson, 1984; Barber, 1984). The ATPase complex has a similar distribution as the PSI complex.

Figure 7 shows the model proposed by Anderson (1984) showing an even distribution of cyt $\underline{b} / \underline{f}$ complex along the membrane. At present most experimental evidence appears to favour this model.

1.6.3 The Protein Complexes of the Thylakoid Membrane. 1.6.3.1 The PSII-LHCII Complex.

A variety of preparations exhibiting PSII activity has been made, ranging from reaction centres to vesicle-like structures. Most of these preparations are capable of supporting electron transport from an exogenous donor to an acceptor but are unable to carry out oxygen evolution. However, Berthold et $\underline{\text { al }}$ (1981), have made an oxygen evolving PSII preparation these membrane fragments are composed of unsealed appressed granal stacks (Dunahay et al,1984). This preparation was characterised by Lam et al (1983) and was shown to be composed of at least 10 polypeptides with molecular weights ranging from 49$10 \mathrm{KDa}$; the most of which have been assigned functions 


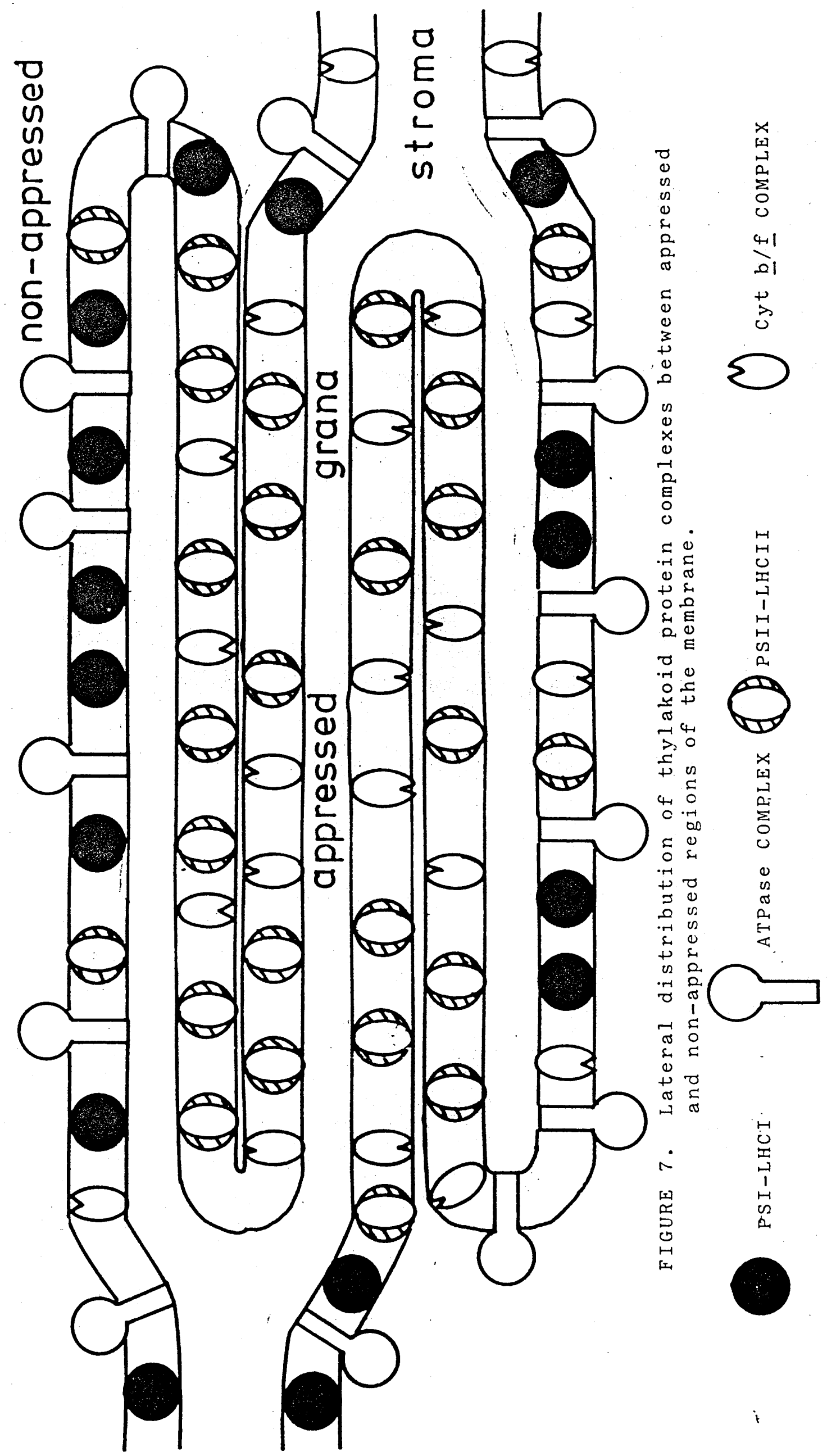




\begin{tabular}{lll} 
POLYPEPTIDE (KDa) & FUNCTION & $\begin{array}{l}\text { COMPONENTS } \\
\text { BOUND }\end{array}$ \\
\hline $\begin{array}{l}\text { 49-41 } \\
32 \text { (Lysine rich) }\end{array}$ & $\begin{array}{l}\text { R.C. complex } \\
\text { PSII protein } \\
\text { shield }\end{array}$ & $\begin{array}{l}\text { Qb. } \\
29,27,25\end{array}$ \\
$33,23,17$ & $\begin{array}{l}\text { Light Harvesting } \\
\text { oxygen } \\
\text { evolving } \\
\text { complex }\end{array}$ & $\left(\mathrm{Mn}, \mathrm{Cl} 1^{-}\right) ?$ \\
10 & unknown & Cyt b 559 \\
\hline
\end{tabular}

The assignation of the 49 and $41 \mathrm{kDa}$ proteins as the reaction centre protein is still tentative.

1.6.3.2 The PSI-LHCI Complex.

Stromal lamellae, which contain relatively little PSII were first isolated over 20 years ago ( Boardman and Anderson, 1964). These preparations contained a large amount of light harvesting chlorophyll molecules, with chlorophyll to $\mathrm{P} 700$ ratios ranging from 80-150. A recent preparation of stromal lamellae (Mullet et al, 1980$)$, has a chlorophyll. to $\mathrm{P} 700$ ratio of 100 , and was composed of a minimum of thirteen polypeptides with apparent molecular weights ranging from 5-65KDa. The proposed function of some of these polypeptides is shown in the table below. 


\begin{tabular}{lll} 
POLYPEPTIDES (KDa) & FUNCTION & $\begin{array}{l}\text { COMPONENTS } \\
\text { BOUND }\end{array}$ \\
\hline $\begin{array}{l}\text { Reaction } \\
\text { Centre }\end{array}$ & $\begin{array}{l}\text { P700 aná } \\
\text { Primary } \\
\text { acceptor } \\
\text { of PSI } \\
\text { Chla and b }\end{array}$ \\
\hline
\end{tabular}

A PSI preparation with a chlorophyll to P700 ratio of 40 was made by Bengis and Nelson (1975), (this contained five polypeptides with molecular weights: $70,25,20,18$ and $16 \mathrm{KDa}$ ) which was capable of NADP photoreduction provided ferredoxin, ferredoxin NADPreductase and plastocyanin were added.

Recently it has been shown that PSI has an antenna complex specifically associated with it, and which has been named light-harvesting complex I (LHCI). Three lines of evidence suggested the existence of this complex: 1) Mullet et al (1980) extracted a PSI particle, designated PSI-110 indicating the ratio of chl:P700, which exhibited two peaks in the chlorophyll fluorescence emmission spectrum, reflecting the presence of two chlorophyll-protein populations. Removal of polypeptides having molecular weights between 19-24kDa from this particle, resulted in a chl:P700 ratio of 65 and the loss of the long wave length fluorescence peak; 2) Haworth et al (1983) showed that $10 \%$ of the $\mathrm{Ch} 1$ associated with PSI-110 was Chlb. Degradation of this preparation to PSI-65 results in a parallel loss of chl b, longwave fluorescence and a group of polypeptides with molecular weight of 19-24 KDa; and 3) Lam et al (1984) extracted a chl b binding protein with a molecular weight of about $20 \mathrm{kDa}$. This polypeptide showed no immunological reactivity with antibodies to the PSII Chl a/b protein. 
A topographical model for PSI organisation in the thylakoid membrane has been proposed by oritz et al (1985). This model emphasises the transmembrane organisation of each peptide component of the PSI complex and this is further supported by Ryrie et al (1985).

1.6.3.3 The Cytochrome $\underline{b} / \underline{f}$ Complex.

This is a non-pigmented protein complex which mediates electron transfer between the two.

photosystems: electrons are received into the complex from plastoquinone and are passed out onto the primary donor of PSI (P700). The manner in which electrons are handled by the components of this complex is still not clear (see section 1.4.7).

The Cyt $\underline{b} / \underline{f}$ complex was first isolated and identified by Nelson and Neumann (1972). Following this Hurt and Hauska (1981) isolated a complex which was composed of four polypeptides with molecular weights of $34 / 33,23,20$ and $17.5 \mathrm{KDa}$. The functional components associated with this complex are two cyt b.6, one cyt $\underline{f}$ and the Reiske iron-sulphur centre. The polypeptide and redox component relationship is shown in the table:

$\begin{array}{cc}\text { POLYPEPTIDE (KDa) } & \text { REDOX COMPONENT } \\ 33 / 34 & \text { cyt' } 1 \\ 23 & \text { cyt } \underline{b}_{6} \\ 20 & \text { Reiske Fe-S } \\ 17 & -\end{array}$


The stoichiometry of the polypeptides is believed to be $1: 1: 1: 1$ for the $33,23,20$ and 17 KDa respectively. However there is a suggestion that a ratio of $1: 2: 1: 2$ may be more correct (Hauska et $\underline{\text { al }}$,1983). The structure of the functional unit of this complex in the membrane is not known although some experimental findings (see Hauska et $\underline{\text { al }}$, 1983) support a dimeric structure having a molecular weight of 500-550 KDa.

This complex spans across the thylakoid membrane and recent evidence for this arrangement is given in section 1.7 .

1.6.3.4 The ATPase Complex.

The ATP enzyme complex is anisotropically arranged across the thylakoid membrane. Two distinct components can be distinguished, (1) $\mathrm{CF}_{\mathrm{O}}$ : the hydrophobic membrane-bound portion which forms a proton channel across the membrane and (2) $\mathrm{CF}_{1}$ : the catalytic, extrinsic part which is hydrophillic and protrudes out from the membrane into the stroma (Nelson, 1981).

The $C F_{\mathrm{o}}-\mathrm{CF}_{1}$ complex is composed of eight different polypeptide sub-units. Five of these make up $\mathrm{CF}_{1}$ and have been designated: $\boldsymbol{\alpha}(59 \mathrm{KDa}), \boldsymbol{\beta}$ (56 KDa), $\gamma(37 \mathrm{KDa}), \delta(25 \mathrm{KDa}), \boldsymbol{\varepsilon}(13.5 \mathrm{KDa})$. The stiochiometry of these sub-units has been proposed to be 3 a : $3 \beta=1 \gamma: 1 \delta: 1 \varepsilon$, as found for the coupling factor of E. Colii (Nelson, 1981). The remaining three polypeptides comprise the CFo and have been designated: I (18KDa), II (14KDa), III ( $8 \mathrm{KDa})$. The stoichiometry of these sub-units has not yet been established in the chloroplast $\mathrm{CF}_{0}$. 
The five sub-units of the $C_{1}$ complex have been shown to have specific functions. The a sub-unit is believed to have a regulatory role in catalysis while the active site is located in the $\beta$ sub-unit. $\mathrm{CF}_{1}$ is bound to the membrane by the $\delta$ sub-unit (Nelson, 1976) and the $\varepsilon$ sub-unit has been assigned an inhibitory role (Nelson et al ,1972). The $Y$ sub-unit appears to have a central function in the regulation of proton movements: it may either mediate proton movements within the enzyme or mediate charges induced by protons (McCarty and Carmeli, 1982). Interactions occur between the sub-units with the $\gamma$ sub-unit having a central position from where it interacts with all the other sub-units. The $\gamma$ sub-unit has also been implicated in the formation of $\mathrm{CF}_{1}$, where it may act as a template (Nelson et al 1980).

\subsection{PROOTEEOLY}

Proteolytic digestion of thylakoid membranes has been widely used to investigate the structure and function of the thylakoid membrane. This experimental approach has yielded information on the topology and functions of PSII, LHCP-II, and the cyt $\underline{b} / \underline{f}$ complex.

Proteolytic enzymes are useful tools to investigate the topography of the membrane because they are large molecules and cannot pass across the membrane. Therefore, provided moderate concentrations of enzymes are used, general disruption of the membrane will not occur, and the enzyme can be used as a specific probe on the outside of the membrane. 
PHOTOSYSTEM II - the effects of trypsin on the functions of PSII were studied in detail by Renger (1976) and Renger et al (1976). This led to the identification of a polypeptide which is situated at the outside of the thylakoid membrane and has binding sites for $Q b$ (see 1.4.3) the secondary acceptor of PSII and for the triazine and urea herbicides (Tischer and strotmann, 1979). This protein is essential for the functioning of linear electron transport and has subsequently been identified as a $32 \mathrm{kDa}$ protein (Mulle't and Arntzen, 1981).

Recently a technique has been developed which has allowed the isolation of inside-out thylakoid vesicles (Andersson and Akerlund, 1978). This has been lised to expose the inner side of the thylakoid membrane to trypsin. These results have shown that the proteins exposed on the inside of the membrane are different from those on the outside and that three polypeptides $(33 \mathrm{KDa}, 23 \mathrm{KDa}$ and $16 \mathrm{KDa}$ ) are implicated in the water splitting capacity of PSII (Akerlund and Jansson, 1983)).

THE LIGHT HARVESTING COMPLEX ASSOCIATED WITH PSII extremely mild proteolytic (trypsin) digestion of thylakoid membranes results in the loss both of membrane stacks and of the cation-induced transfer of energy from PSII to PSI (Steinbeck et al,1979). These functional changes occurred concomitant with the removal of a $2 \mathrm{kDa}$ portion of the $25 \mathrm{KDa}$ light harvesting protein associated with PSII. These data suggest that a surface-exposed portion of the LHC-II was irvolved in membrane stacking and in the regulation of energy distribution between PSII and PSI (Steinbeck et al,1979; Carter and Staehlin, 1980). 
THE Cyt b/f COMPLEX - the distribution of cytochrome across the membrane was investigated using right-side out and inside-out thylakoid vesicles (Mansfield and Bendall, 1984). The study suggested that the haem groups of cyt $\underline{b} 559$ cyt $\underline{b} 563$, and cyt $\underline{f}$ are buried within the membrane as none of these were affected by trypsin or pronase. However, the apoprotein of cyt b559 appears to be more exposed on the outside

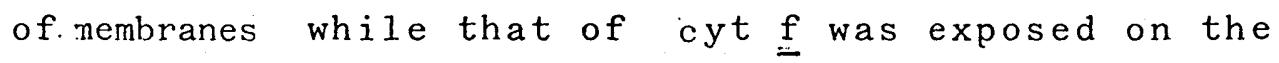
lumenal side. However, willey et al (1984) showed that a portion of cyt $f$ was exposed on both the stromal and lumenal side of the membrane. More recently a proteolysis study, using pronase E, has shown that all four polypeptides of this complex (see section 1.6) span the membrane (Oritz and Malkin, 1985 ).

\subsection{EXPERIMENTAL_RATIONALE}

The use of proteolytic enzymes has yielded many important findings about the structure and function of the thylakoid membrane (see 1.7). In this research the effects of the serine proteases, trypsin and chymotrypsin have been compared. The rationale behind using these particular enzymes is that they have a similar structure, molecular weight, pH optimum and the same inhibitor can be used to stop their reactions while they have different specificities for the bonds they attack. This allows all the parameters to remain the same and to observe changes due to the change in enzyme only.

Speceificty - Trypsin cleaves bonds involving arginine or lysine residues; these are aliphatic amino acids that have a positive charge. chymotrypsin cleaves bonds involving aromatic amino 
acids e.g. phenylalanine, tyrosine and tryptophan and bulky uncharged aliphatic amino acids e.g. asparagine or methionine. It was hoped that trypsin and chymotrypsin would alter different functions and that this could be correlated with the differences in specificities.

\subsection{THEORY OF EXPERIMENTAL METHODS.}

\subsubsection{Flash Absorption Spectrophotometry.}

1.9.1.1 The electrochromic bandshift at $515 \mathrm{~nm}$ ( $\Delta \mathrm{A} 515$ )

This absorption shift was first observed by Duysens (1954) using flash absorption spectrophotometry on the green unicellular algae chlorella. The lightminus-dark difference spectrum showed a maximum at $515 \mathrm{~nm}$ and minima at 478 and $420 \mathrm{~nm}$; at that time the pigments involved were unknown. A similar absorption change was observed in chloroplasts (Junge and witt, 1968) which had a maximum of $515 \mathrm{~nm}$ and a minimum at $480 \mathrm{~nm}$. These changes are now thought to be due to an electrochromic effect of the membrane pigments.

Electrochroism (Platt, 1961) is the effect of strong electric fields $\left(10^{7} \mathrm{Vm}^{-1}\right)$ on the absorption spectrum of dye molecules, the major change being a homogenous shift of an absorption band by a few nanometers.

When a quantum of light is absorbed by pigments e.g. in the chloroplast membrane, the constituent molecules are raised from low-to high-energy states. If an electric field is present it will interact with these energy states changing the ground and excited state energy levels thereby bringing about a change in the 
absorption spectrum of the molecules. The bandshift effect is therefore based on the potential energy of permanent and induced molecular dipole moments in the electric field. This effect can be expressed mathematically:

$$
\text { h } \Delta V=-\left(\mu_{e}-\mu_{g}\right) \vec{F}-\frac{1}{2}\left(\alpha_{e}-\alpha_{g}\right) \vec{F}^{2}
$$

Where $\mu_{e}$ and $\mu_{g}$ are the permanent dipole moments, $\alpha_{e}$ and $\alpha$ are the polarizabilities of excited and gound states and $F$ is the field (Junge and witt, $1968)$.

Three lines of evidence have linked the absorption changes, $\Delta A 515$, with an electrochromic response of carotenoids and chlorophylls to a light-induced electric field across the thylakoid membrane.

1) Kinetic Evidence

After flash excitation of chloroplasts a fast rise phase of $<20 \mathrm{~ns}$ is seen which $\cdots$ subsequently decays in the dark in the time range of 0.01 to 1 second (Junge and Witt, 1968), (see Fig. 8). The fast rise phase has been attributed to primary crarge separation occurring at the reaction centres of PSI and PSII which occurs on a similar time scale (wolff et al, 1969). Inhibition of either reaction centre inhibits $\Delta$ A5 15 by $\sim 50 \%$ indicating that both contribute, about equally, to the absorbance change.

Acceleration of the dark decay can be observed under a number of conditions: ageing, osmotic shock and the presence of solvents or ion transporting antibiotics (Junge and Witt,1968); under phosphorylating conditions an increase in the rate of decay was observed by Rumberg and siggel (1968) and they associated this with an 


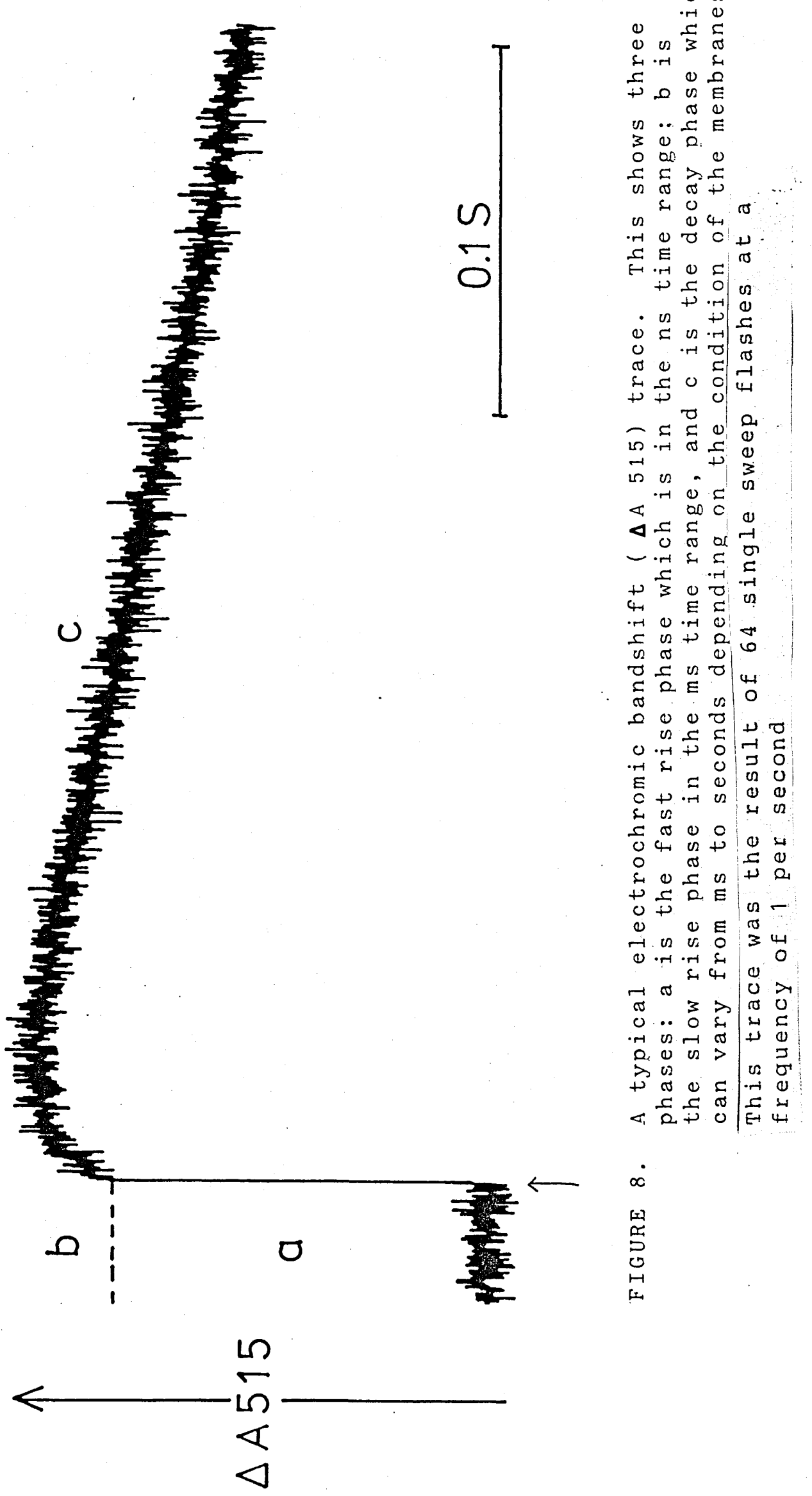


increase in proton flux through the coupling factor; moreover removal of the coupling factor also results in a rapid rate of decay.

\section{2) Spectroscopic Evidence}

Model systems were prepared and comparisons made between these and the in vivo system. The models consisted of layers of photosynthetic pigments built up on glass slides across which an electric field was applied (Schmidt et al,1971), this produced an absorption spectrum which was similar to the light-induced in vivo spectrum. In this way it was found that both chlorophyll b and carotenoids are involved in the electrochromic effect of higher plants.

\section{3) Electrica1 Evidence}

a) Diffusion potentials

Diffusion potentials were induced in chromatophores from Rhodopseudomonas sphaeroides and the extent of the absorption changes were seen to vary linearly with the diffusion potential (Jackson and Crofts, 1969). Analogous studies have been done on chloroplasts but the earlier work proved to be less clear due to light scattering artifacts. However more recently Schapendonk and Vredenberg (1977) showed a similar linear relationship between the extent of the absorption change and the diffusion potential in chloroplasts.

b) Macroscopic Electrodes

These were introduced by Fowler and Kok (1.974) and subsequently used by witt and zickler (1974) who showed that the electrochromic absorption change induced by 1 ight could be correlated linearly with the voltage measured by electrostatic induction. 
c) 'Blebs'

More recently osmotically swollen chloroplasts

(blebs) have shown that the spectra of the absorption changes at $515 \mathrm{~nm}$ induced in the dark by electric fields are identical to those generated by $1 \mathrm{ight}$ (de Grooth et a르,1980).

THE RELATIONSHIP OF THE ELECTROCHROMIC BANDSHIFT TO VOLTAGE

Evidence has been accumulated suggesting that the electrochromic bandshift is linearly related to voltage across the membrane. Jackson and Crofts (1969) showed a linear relationship between the carotenoid bandshift and the membrane potential, using pulses of $\mathrm{KCl}$ added to chromatophores in the presence of valinomycin. Similar experiments were done on thylakoids by Schapandonk and Vredenberg(1977). Two further lines of evidence gave support to the existence of this relationship in thylakoid membranes: the ionophore almethicin forms pores in black lipid membranes which are dependent on the presence of a potential difference across the membrane- this method has been used to correlate voltage with absorption changes; the number of charges translocated across the membrane in one turnover of the electron transport chain was measured and this gave a good correlation with the extent of the bandshift (Witt, 1978).

Absorption changes in vivo give a linear function of the electric field while those in vitro give a quadratic function as would be expected for symmetrical carotenoids. Schmidt et al (1971) explained this observation by assuming that carotenoids in vivo are exposed to a permanent electric field which is much stronger than the lightinduced one. It was demonstrated by Reich and sewe (1977) that in the presence of such a permanent field a pseudo-linear electrochromic response can be seen, even though the real response is quadratic. 
In addition to the fast rise kinetics of the $\Delta A 515$, Joliot and Delosme (1974) observed a slow rise phase in algae. They called the fast rise phase a and the slow rise phase b (see Fig. 8). The light-minus-dark difference spectrum of the slow rise is the same as that for the fast $r$ ise phase and therefore is also thought to be due to an. electrochroism of the pigments in the membrane.

The origin of the slow phase in chloroplasts is not unequivocal. However in Rhodopseudomonas sphaeroides it was shown that two slow phases exist in the rise of the carotenoid bandshift (Jackson and Dutton, 1973). The first phase rises with a halftime of $0.15 \mathrm{~ms}$ and may be due to photo-oxidation of cyt $c$ and the re-reduction of $\mathrm{p}^{+}$. A much slower phase with a half-rise time of 5 ms (possibly corresponding to the slow phase in chloroplasts)has been correlated with the oxidation of cyt b 563 and the reduction of cyt c (Jackson and Dutton, 1973; Prince et al, 1978$)$.

In chloroplasts an extra electrogenic step has been proposed either in an $\mathrm{H}^{+}$-transporting Q-cycle (Velthuys, 1979; 1980) or in cyclic electron transport around PSI (Crowther and Hind,1980). 01 sen and Barber (1980) suggest that the slow component appears as a consequence of the delocalisation of charge due to the release of $\mathrm{H}^{+}$and $\mathrm{OH}^{-}$into the aqueous phase during electron transport reactions. Finally Vredenberg and Shapendonk (1981) and Schuurmans et al (1981) suggest a different model: the slow rise phase is thought to be a reflection of localised inner membrane conformational changes probably involving the ATPase, these changes being accompanied by changes in strength and orientation of local membrane fields. 
The magnitude of this slow phase can be reduced by addition of valinomycin (Schapendonk and vredenberg, 1979 ) and increased by nigericin (Crowther and Hind, 1980). A reduction was also seen with adenine nucleotides (Girault and Galmiche, 1978) and by substances that alter the ATP synthesising complex (Schuurmans et a1,1981; Vrederiberg and Schapendonk, 1981). Although intensive experimentation has produced a number of hypotheses as to the origin of the slow phase none,has proved to be totally satisfactory. However nore recently experimental data has suggested that an extra electrogenic step involving a Q-type cycle (Joliot and Joliot, 1984), linked to cyclic electron transport (crowther and Hind, 1980; Moss and Bendall, 1984), may be the source of the slow phase.

\section{LIMITATIONS}

Care has to be taken in the interpretation of these changes. A number of other phenomena can cause absorption changes at $515 \mathrm{~nm} 1$ ) carotenoid triplet formation $\quad$ 2) light scattering changes. Normally these effects can be separated kinetically: carotenoid triplets decay in $\mu$ s time range while light scattering changes come into play $>$ one second, therefore by using flash conditions and observing changes at $<500$ ms the changes should be due to electrochroism alone (Junge, 1977).

1.9.1.2. Light Induced 0xonol. VI Absorbance Changes.

oxonol VI is one of a group of dye molecules that can be used as an extrinsic probe of membrane potential. 
Changes in the absorbance of oxonol VI are measured around $600 \mathrm{~nm}$, a wavelength where chloroplast absorbance is low. When oxonol VI reacts with proteins and lipoproteins there is a red shift of its absorption band - the absorbance changes are related to the number of dye molecules which are bound (Galmiche and Girault, 1981).

Bashford et al (1979a) studied the ineractions of a series of oxonol dyes on phospholipid dispersions. Three major factors affecting the interaction of oxonols were 1) the structure of the dye 2) the physical chemistry of the vesicle 3 ) the ionic strength of the medium. In addition to these major factors the chemical composition of the membrane is also thought to be jmportant.

Light-induced shifts of absorption spectra were seen on adding oxonol VI to chromatophores of bacteria (Bashford et al ,1979). The oxonols appeared to respond to 1 ight-induced energization of the chromatophore membrane by shifting their absorption maxima. From experiments using valinomycin $/ \mathrm{K}^{+}$and nigericin (Bashford et a ․ 1979; Galmiche and Girault, 1981 and Schuurmans et al, 1978) it was concluded that the oxonols respond to the light-induced membrane potential. These dyes are anions at neutral pH values and probably distribute across the membrane in accordance with the potential, which is positive to the inside of both thylakoids from plants and chromatophores from bacteria. This influx of anions (oxonol) partly discharges the membrane potential which can be seen from the increase in rate of decay of $\Delta A 515$. The response of oxonol VI is much slower than for the intrinsic electrochromic band shift and therefore can be used only to monitor slow changes or steady state 
levels of membrane potential. In addition since oxonol VI dissipates the field, low concentration $(\sim 1 \mu \mathrm{m})$ must be used.

\subsubsection{Chlorophyll Fluorescence.}

Light absorption by the antenna pigments (see 1.3) is the first process to occur in photosynthesis. The energy absorbed in this way raises the electrons within these pigments from a ground state to an excited state. This excited state is unstable having a lifetime of $\sim 10^{-9}$. Four dissipative processes compete for this energy: 1) light emission, (fluorescence); 2) radiationless transition (heat); 3) intersystem energy transfer from PSII to PSI; 4) photochemistry. The chlorophyll fluorescence yield of photosystem II can be defined as the ratio of the fluorescence rate constant to the sum of all the de-excitation. rate constants. (Clayton, 1965)

$$
\phi_{f}=K_{f} /\left(K_{f}+K_{t}+K_{h}+K_{p} Q\right)
$$

Where $\phi_{f}=$ quantum yield of fluorescence, and the rate constants associated with the decay process of $\mathrm{K}_{\mathrm{f}}=$ fluorescence, $\mathrm{K}_{\mathrm{t}}=$ transfer of $1 \mathrm{ight}$ energy from PSII to PSI, $K_{h}=$ heat loss, $K_{p} Q=$ photochemistry. It can be seen that if any of the rate constants change then the fluorescence yield will also change.

Fluorescence measurements can be used to follow the primary processes of PSII. Duysen and Sweers (1963) showed that the fluorescence intersity was dependent on the redox state of the primary acceptor (Q) of PSII. When $Q$ is reduced $\left(Q^{-}\right)$, and if no re-oxidation occurs, fluorescence reaches a maximum, conversely when $Q$ is in the oxidised state fluorescence is quenched?. 
When $Q=1$ all traps are open and $\phi_{f}$ is 1 ow:

$$
\phi_{f}=K_{f} / K_{f}+K_{t}+\dot{K_{h}}+K_{p}^{1}
$$

but when $Q=0$ all traps are closed and $\phi f$ is high:

$$
\phi_{f}=K_{f} / K_{f}+K_{t}+K_{h}
$$

There is thus an inverse relationship between

fluorescence and photochemistry: when fluorescence is high, photochemistry is low.

Fluorescence induction curves can be used to assess the photochemical efficiency of PSII. When dark adapted chloroplast samples are exposed to light the initial fluorescence rises to a level, $F_{0}$ (this fluorescence is constant and is not related to the redox state of $Q$ ) as $Q$ becomes reduced the reaction centre traps close and fluorescence rises to a maximum level, Fm. But intersystem transfer of electrons will re-oxidise $Q$ and consequently the fluorescence level may vary. To prevent this variation DCMU, a herbicide which inhibits electron transfer between $Q \mathrm{a}$ and $\mathrm{Qb}$, is added to the sample and the fluorescence rises rapidly to a maximum level and remains steady. By deducting $F_{o}^{\prime}$ from $F_{m}$ a parameter $F_{v}$ (variable fluorescence) is obtained, and this is the fluorescence which occurs as the PSII traps close. It is possible to use chlorophyll fluorescence induction curves to estimate the photochemical efficiency of PSII; if $\mathrm{F}_{\mathrm{o}}$ : and $\mathrm{F}_{\mathrm{m}}$ are measured.

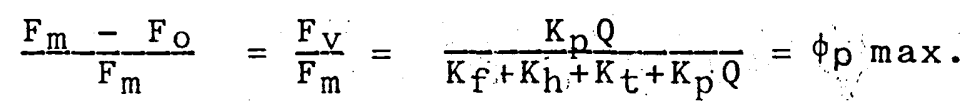

Фpmax is the maximum photochemical efficiency of PSII and has a value between 0 and 1: the closer to 1 the higher the efficiency of PSII. (Malkin and siderer, 1974). However, discrepancies have been noted between the measured and predicted levels of $F_{m}$ and $F_{o}$ and to 
account for this it has been assumed that an extra radiationless de-activation pathway occurs in closed reaction centres (Van Grondelle and Duysens, 1980) Haenel et al, 1982).

The expression $\frac{F_{V}}{F_{m}}=\phi_{p}$ max should be modified to

$$
\frac{F \dot{v}}{F m}=\phi_{p} \max \times \frac{K_{p}}{K_{p}-x \cdot K d}
$$

Where $K_{d}$ is the rate constant for a further radiationless de-activation step. (Van Grondelle and Duysens, 1980) 
CHAPTER TWO - MATERIALS AND METHODS. 
MATERIALS AND METHODS.

\subsection{GROWTH CONDITIONS.}

Peas (Pisum sativum var. Little Marvel) were grown in trays of vermiculite for 10-14 days at a temperature of $23^{\circ} \mathrm{C}$. The light was provided by, mercury vapour lamps $\left(15 \mathrm{wm}^{-2}\right)$ with a $12 \mathrm{~h}$ photoperiod.

\subsection{ISOLATION OF CHLOROPLASTS.}

$50 \mathrm{~g}$ of peas was homogenised in $200 \mathrm{ml}$ of a medium containing: $0.33 \mathrm{M}$ Sorbitol, $50 \mathrm{mM} \mathrm{KH}_{2} \mathrm{PO}_{4}, 5 \mathrm{mM} \mathrm{\textrm {MCl } _ { 2 }}$, $0.1 \%(\mathrm{w} / \mathrm{V}) \mathrm{NaCl}$ and $2 \%(\mathrm{w} / \mathrm{v})$ sodium iso-ascorbate, pH 6.5. Grinding was at high speed for 5 seconds in a polytron (Homónèiser TypePT 1 $0-35 \mathrm{Kinematica,}$ Switzerland). The homogenate was filtered first through 2 then through 8 layers of muslin and a thin layer of cotton wool. The filtrate was centrifuged at $2200 \times \mathrm{g}$ for 90 seconds in a bench top centrifuge (MSE- super minor) yielding a pellet of chloroplasts. osmotically shocked chloroplasts were prepared by resuspending the pellet in $2 \mathrm{mM} \mathrm{MgCl}, 10 \mathrm{mM} \mathrm{KC1}$ and 20mM Tricine-NaOH,pH 7.4. The chloroplasts were routinely stored in the dark on ice for a minimum of one hour before use (Stokes and walker,1971)

\subsection{DETERMINATION OF CHLOROPHYLL CONCENTRATION.}

Chlorophyll concentration was determined spectrophotometrically: $25 \mu \mathrm{l}$ of the chloroplast suspension was added to $10 \mathrm{ml}$ of $80 \%(\mathrm{v} / \mathrm{v})$ acetone; the solution was then filtered through a Watmans No: 1 paper and the 
absorption measured at 645,654 and $663 \mathrm{~nm}$, using a scanning spectrophotometer (Pye-unicam SP8-500). The concentration of chlorophyll was determined using the method of Arnon (1949):

$$
\begin{aligned}
& \mathrm{A} 663=82.04 \mathrm{Ca}+9.27 \mathrm{Cb} \\
& \mathrm{A} 645=16.75 \mathrm{Ca}+45.6 \mathrm{Cb}
\end{aligned}
$$

Solving for Chla and b using simultaneous equations

$$
\mathrm{C}=20.2 \mathrm{~A} 645+8.02 \mathrm{~A} 663
$$

correcting for the dilution factor gives an answer in $\mu \mathrm{gChl} \mathrm{ml}^{-1}$.

The intersection of the chla and Chlb peaks is at $652 \mathrm{~nm}$ and the specific absorption co-efficient at this wavelength is 34.5 therefore:

$$
\mathrm{c}=\frac{\mathrm{A} 652 \times 1000}{34.5}=\mathrm{mg}^{-1}
$$

\subsection{EXPERIMENTAL PROCEDURES.}

\subsubsection{Enzyme Incubation Procedure.}

Trypsin, type XIII (N-Tosyl - phenylalanyl chloromethyl ketone treated to exclude chymotrypsin activity) and chymotrypsin, Type VII (Tosyl-lysylchloromethyl ketone to exclude trypsin activity) were purchased from Sigma chemical Company.

The enzymes were prepared as solutions in $20 \mathrm{mM}$ tricine buffer pH 7.4, at $x 100$ the required concentration, and were stored as $1 \mathrm{ml}$ aliquots in the freezer. Enzyme solutions were never re-frozen as this had a detrimental effect on enzyme activity (section 2.4.2). 
The enzyme incubation procedure was as follows: the stock solution of enzyme was diluted to the required concentration in the assay buffer (details in legends of figures), and divided into small (0.5-4 ml) aliquots. The tubes containing these samples and the control samples (buffer containing no enzyme) were equilibriated at $23^{\circ} \mathrm{C}$ for a minimum of 5 min. Chloroplasts were then added to the. required concentration (see figure legends). After the incubation period was complete soy bean trypsin inhibitor (sigma Chemical Company) was added to stop the reaction. Immediately following this any necessary co-factors were added and the required measurement made.

The 1 ight source used for enzyme incubation in the light was a microsope lamp (Watson Barnet) giving white light with an intensity of $120 \mathrm{wm}^{-2}$.

\subsubsection{Enzyme Assays.}

The activity of the enzymes was assayed spectrophotometrically as indicated by schwert and Takenaka,(1955). Chymotrypsin in $1 \mathrm{mM} \mathrm{HCl}(0.2 \mathrm{mI})$ was added to $3 \mathrm{ml}$ of acetyl tyrosine ethyl ester (ATEE) $\left(10^{-3} M\right)$ in phosphate buffer (0.05M) pH 6.5. The activity in this sample was measured against a blank containing phosphate buffer and ATEE $E_{i}$ at $237 \mathrm{~nm}$ where a decrease in absorption occurred due to hydrolysis of the ester.

Trypsin in $1 \mathrm{mMHCl}(0.2 \mathrm{ml})$ was added to $3 \mathrm{ml}$ of benzoyl arginine ethyl ester $\left(10^{-3} \mathrm{M}\right)$ in phosphate buffer $(0.65 \mathrm{M}) \mathrm{pH} 7.0$. The activity was measured at $253 \mathrm{~nm}$ where an increase in absorption was brought about by hydrolysis of the ester. 
The activity in these assays was measured over 90 seconds. These assays were used to check that no deterioration in the activity of the enzymes occurred from day to day. In addition the presence of trypsin activity in the chymotrypsin was tested and vice versa. No observable activity was seen in either case this indicating that there was less than $1 \%$ contamination. The trypsin inhibitor will inhibit $1-3 \mathrm{mgml}^{-1}$ of trypsin per mg of inhibitor. In general at least a 20 fold excess was used ('see legends for details)

2.4 .3 Protein Assay.

A tannin assay was used to measure the concentration of protein in the samples prior to gel/electrophoresis. Bovine serum albumin (BSA) was used as a standard: a series of samples were prepared containing from $10-150 \mu \mathrm{g}$ of $\mathrm{BSA}$. The required amount of $\mathrm{BSA}$ was put into each tube and the volume made up to $1 \mathrm{ml}$ with water, these samples were equilibriated for $1 \mathrm{~min}$, at $30^{\circ} \mathrm{C}$, after which $1 \mathrm{ml}$ of tannin medium ( $1 \mathrm{M} \mathrm{HCl} 10 \%$ (w/v) tannic acid and $0.5 \%(w / v)$ phenol) was added. The samples were incubated for $10 \mathrm{~min}$ at $30^{\circ} \mathrm{C}$, on the completion of which $1 \mathrm{ml}$ of gum acacia $(0.2 \% \mathrm{w} / \mathrm{y}$ was added. The turbidity of the samples was measured at $500 \mathrm{~nm}$ indicating the amount of protein. A graph was then produced plotting BSA concentration against the absorbance reading at $500 \mathrm{~nm}$ (A500). To find the concentration of protein in the experimental samples the absorbance at $500 \mathrm{~nm}$ was measured and the concentration read off from the graph. (Mejbaum-Katzenellenbogen and Drobryszka (1951). 


\section{4 .4 PSII Blocked Chloropiasts.}

To inhibit PSII activity, chloroplasts $\left(25 \mu \mathrm{gml}^{-1}\right)$ were incubated in the 1 ight for $1.5 \mathrm{~min}$ in the presence

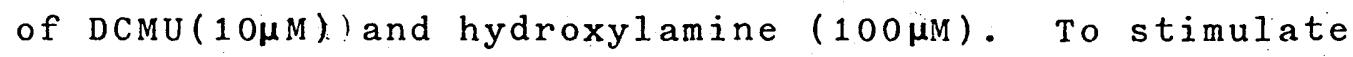
PSI the electron donors TMPD (0.2M) or DCPIP (0.1mM) were added with the reducing agent sodium ascorbate ( $3 \mathrm{mM})$.

\subsection{MEASUREMENT OF OXYGEN EVOLUTION.}

\section{5 .1 The Steady-state 0 Electrode.}

The principle on which the oxygen electrode is based is that of polarography : this technique is used for analysing and studying ions in solution; the concentration of the ions is obtained by using an electrolytic cell and by producing a graph of the current against the potential.

The electrode used was a modified clarke type, (Delieu and Walker, 1972). It comprises a reaction chamber, enclosed by a perspex jacket, separated from the electrode itself by a semi-permeable teflon membrane. Water, the temperature of which was thermostatically controlled, was pumped through the perspex jacket of the electrode and maintained the sample at a constant temperature. A platinum wire embedded in plastic was the cathode while a silver wire bathed in saturated KCI was the anode. The polarity was set up by applying a voltage of $0.65 \mathrm{~V}$ across the two electrodes. $\mathrm{O}_{2}$ was reduced at the platinum electrode:

$$
\mathrm{O}_{2}+\mathrm{H}_{2} \mathrm{O}+4 \mathrm{e}^{-} \longrightarrow 4 \mathrm{OH}^{-}
$$


while the reaction at the silver (+ve) electrode:

$$
4 \mathrm{Ag}+4 \mathrm{Cl}^{-} \longrightarrow 4 \mathrm{AgCl}+4 \mathrm{e}^{-}
$$

The resulting electron flow was proportional to the concentration of $\mathrm{O}_{2}$ in the sample. The rate of $\mathrm{O}_{2}$ being evolved was measured by putting the output from the $\mathrm{O}_{2}$ electrode, which measures the concentration of $0_{2}$, onto a potentionmetric chart recorder.

The scale on the chart recorder was calibrated using maximum and minimum $\mathrm{O}_{2}$ levels obtained from aerated water and $\mathrm{O}_{2}$ free water respectively. The scale set in this manner gives the total span of $0_{2}$ levels and was recorded in units read off the chart recorder paper. The $\mathrm{O}_{2}$ evolved was expressed in $\mu$ moles $0_{2} \mathrm{mg} \mathrm{Chl}^{-1}$ hour ${ }^{-1}$.

\section{5 .2 F1 a}

The electrode usediwas similar to the model described by Joliot and Joliot,(1968). It is composed of two sections separated by a dialysis membrane (Fig. 9): the bottom section houses the platinum electrode whilst the silver electrode is in the top section. A reservoir containing buffer (20mM Tricine, $100 \mathrm{mMKCl}$, $2 \mathrm{mM} \mathrm{MgCl}{ }_{2}$, $100 \mu \mathrm{M} \mathrm{NADPH}{ }_{2}^{\prime}$ ) was connected to the top' section and, by gravity flow; this solution renews the medium between the two electrodes. The sample of algae or chloroplasts (chlorophyli concentration $100 \mu \mathrm{gm}-1)$ wasplaced on the platinum electrode, which was recessed such that the volume of the electrode chamber was approximately $2 \mu l$, when the electrode assembly is pushed upwards against a taut dialysis membrane. The platinum disc was the cathode and the silver electrode (bathed in KCl) was the anode: this polarity was set up by applying $0.65 \mathrm{~V}$ across the two electrodes. (See section 2.5.1). 


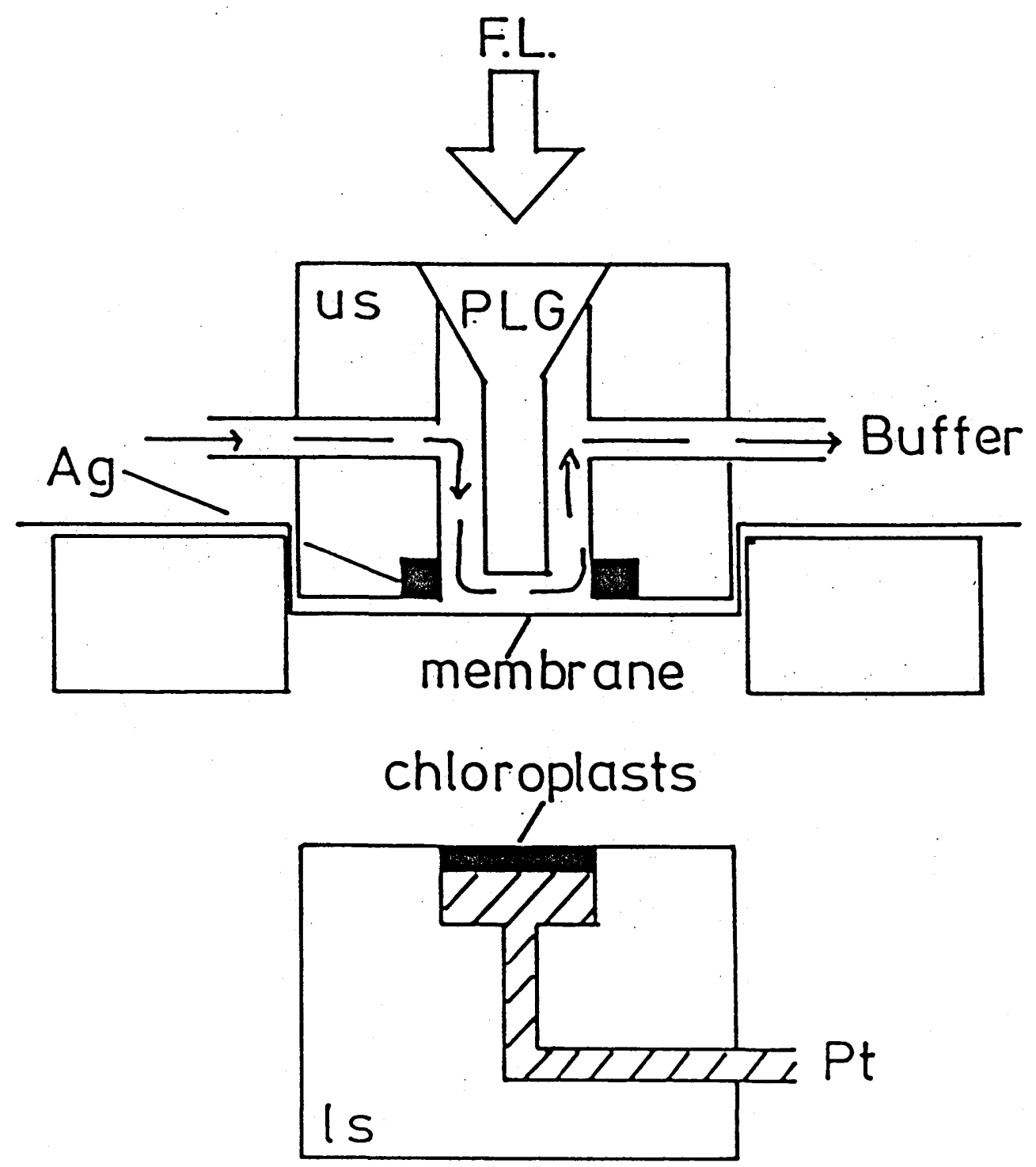

FIGURE 9 .

The flash oxygen electrode. This shows the two sections described in the text. The symbols are: FL, flash lamp: 'PLG, perspex light guide; $\mathrm{Ag}$, silver electrode and $\mathrm{Pt}$, platinum electrode. 
The chloroplast sample was excited by a $5 \mu$ s xenon flash and în order to prevent

the turnover of PSI to be limiting, steady state light was supplied from a microscope lamp (Watson Barnet) protected with a red filter (RG $715 \mathrm{schott).} \mathrm{The}$ flash $0_{2}$ yield wasmeasured by passing the signal from the electrode to a digital transient recorder (Datalab DL901) and then the data was stored and analysed by a Digital Equipment Corporation Computer (DEC PDP-11-34). The equipment set up is shown in Figure 10 .

The flash lamp was triggered by the computer and a series of 23 flashes were given with 0.75 s between each. To make measurements of the light saturation curve, filters were inserted in every 6 th $f 1$ ash allowing three experimental flashes per series of 23 . The data was analysed by counting flashes 2-5, 8-11, 14-17, 20-23 as controls and flashes 6,12 and 18 as experimental. After each flash the $0_{2}$ signal was displayed on an oscilliscope screen along with the signal size. This procedure was repeated for each optical density filter and a saturation curve of $\mathrm{O}_{2}$ yield versus flash intensity was obtained (see Results Chapter 6)'.

The error bars indicated in the graphs are standard errors calculated from the standard deviations from a minimum of four repeats. Figures 61 and 62 show the data from 2 repeats only. 


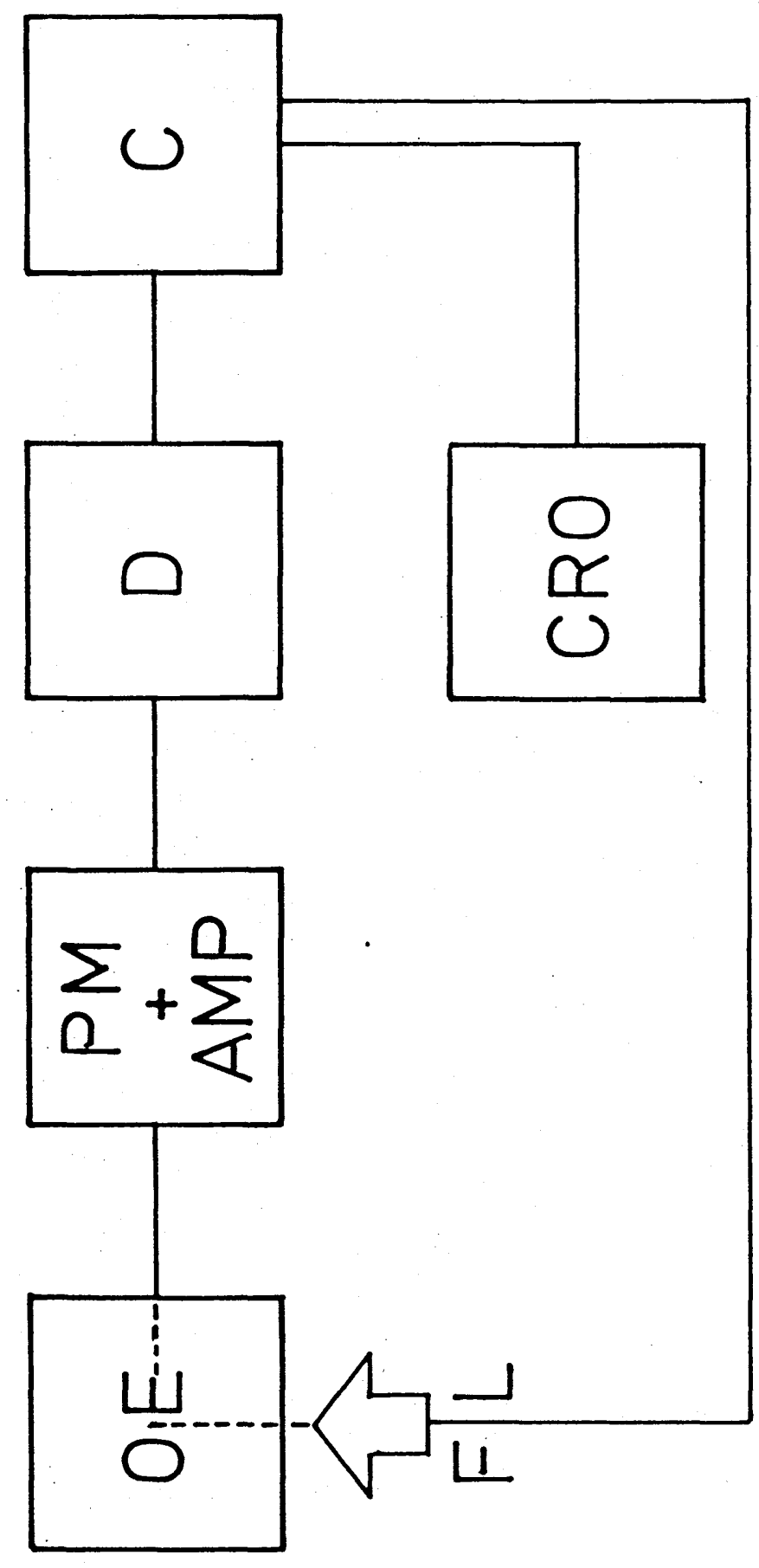

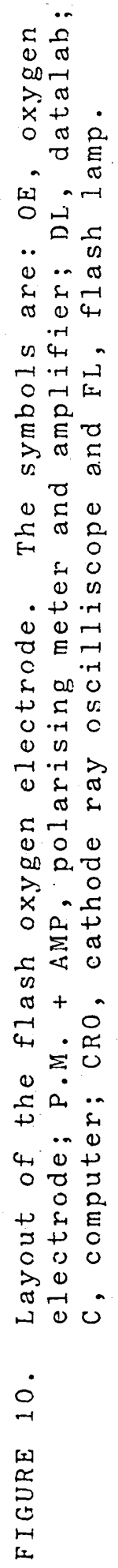




\subsection{MEASUREMENT OF PHOTOPHO SPHORYLATION AND HYDROLYSIS.}

Phosphorylation and hydrolysis measurements were made using the pH-method of Nishimura et al (1962). This method uses the differences in $\mathrm{pK}$ between $A D P$ and $A T P$. When the following reaction takes place, a small change in $\mathrm{pH}$ is brought about:

$$
\mathrm{ADP}+\mathrm{P}_{\mathrm{i}}+\mathrm{nH}^{+} \longrightarrow \mathrm{ATP}+\mathrm{H}_{2} \mathrm{O}
$$

The value of $\mathrm{n}\left(=\mathrm{H}^{+} / \mathrm{P}\right)$ is calculated theoretically, using the Henderson-Hasselbalch equation, and the buffering capacity of the medium $\left(\mathrm{H}^{+} / \mathrm{pH}\right)$ is determined experimentally by titrations. Values of $n$ were taken from Nishimura et al (1962): in the experiments described here a value of $n=0.925$ was used at $\mathrm{pH}$ 7.8. The buffering capacity was measured by adding $10 \mu \mathrm{l}$ of $10^{-2} \mathrm{M} \mathrm{HCl} \quad\left(100 \mathrm{ng} \mathrm{H}^{+}\right.$ions) to each. sample. It is possible to calculate the rate of production or consumption of $\mathrm{H}^{+}$and from this the rates of phosphorylation and hydrolysis can be calculated. A typical phosphorylation trace is shown in Figure'11. The reaction was carried out in a Clarke type $\mathrm{O}_{2}$ electrode where simultaneous measurements of rates of $\mathrm{O}_{2}$ evolution and phosphorylation/hydrolysis could be measured. This method allows control over temperature $\left(23^{\circ} \mathrm{C}\right)$ and constant stirring of the reaction mixture. The light source, from a 150 w quartz-iodine lamp powered by a stabilised D.C. supply (Coutant Electronics) was passed through a water filter and a Wratten 29 red filter. pH changes were measured using a micro-combination pH electrode (EILChertsey, surrey) connected to a pH meter (MSE spetroplus):, the sensitivity of this was increased 


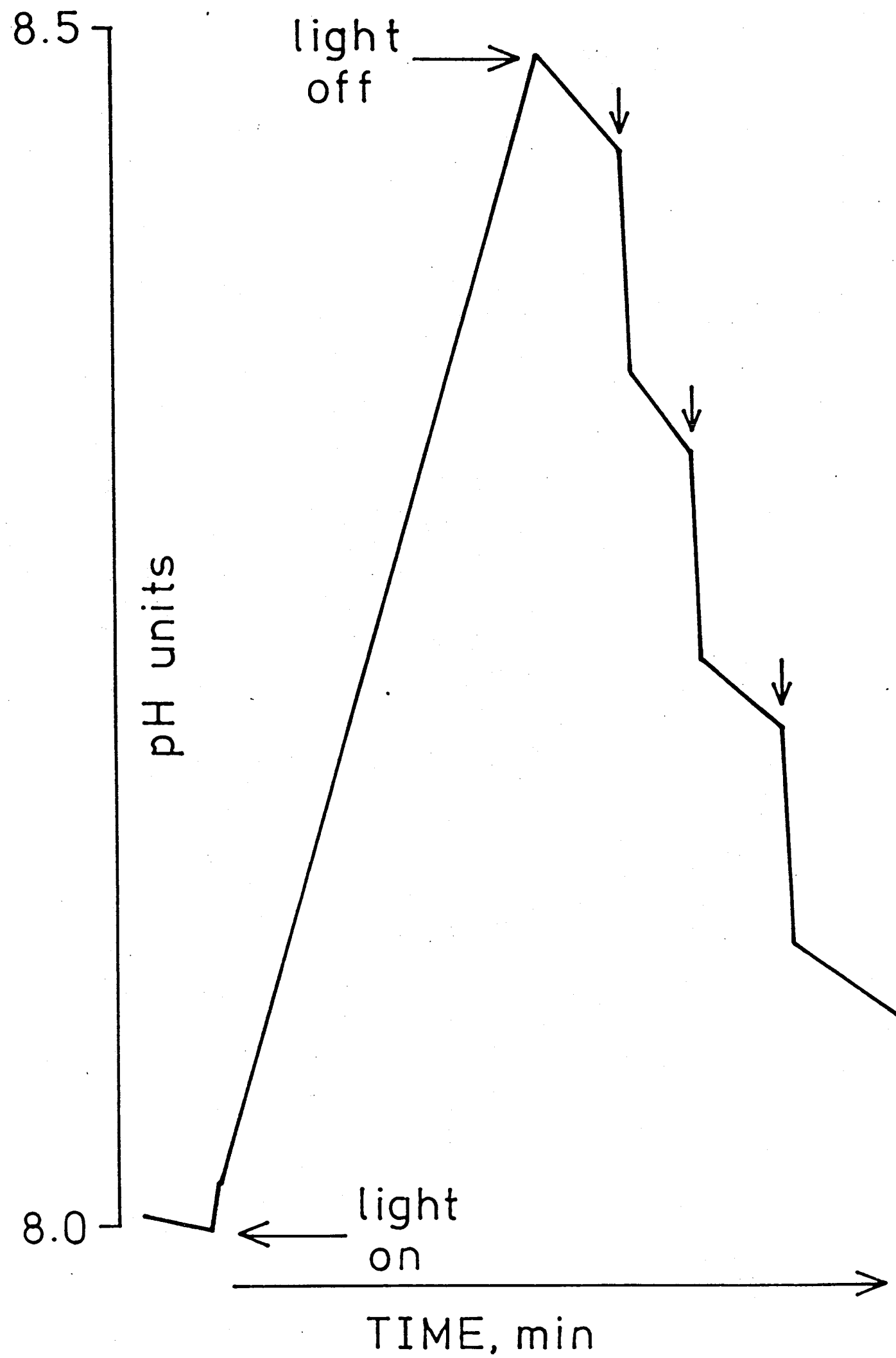

FIGURE 11. A typical phosphorylation trace.

$(\downarrow)$ indicates the addition of HCL. 


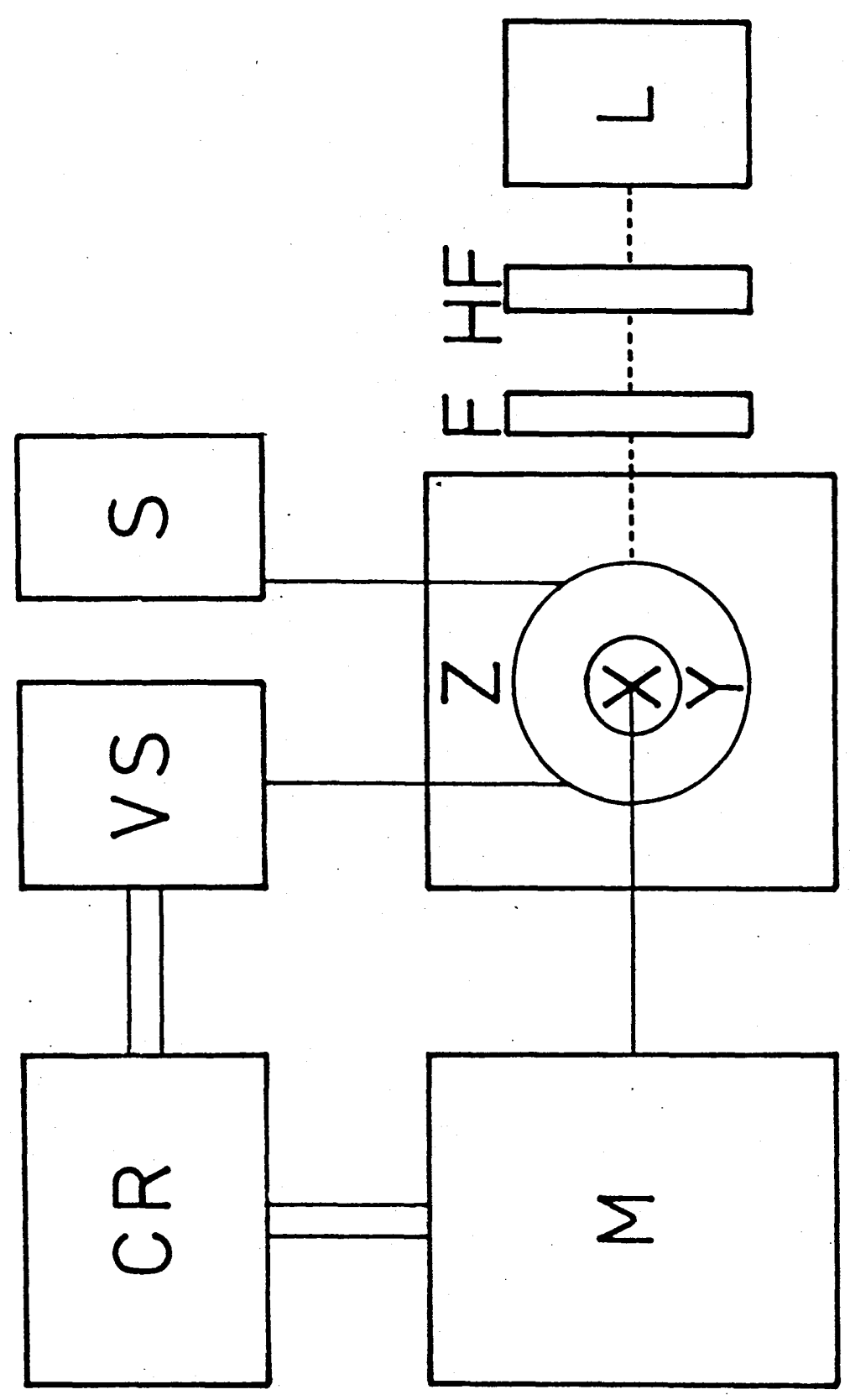

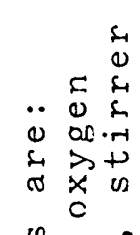

त

$0>1$

E ...

is 0

$+2$

ट. न

E्ञ

$\nabla 0$

- $0 \pi$

Uद 4

$\pm \quad r$

of ${ }^{a}>\dot{x}$

$E$

U... a

\& \& e

$\supset 0>\infty$

v + a

w $\ldots \ldots$

$\begin{array}{lll}0 . H & \text { L } & 5 \\ \text { E } & 0 & 0\end{array}$

- 0

$5+5$

$\begin{array}{lll}1 & 0 & 0 \\ 0 & 0 & 0\end{array}$

r

幽 10

$\underset{-1}{\infty} \cdot \frac{1}{0}$

入ा i

का 讷

c...

$20 \mathrm{c}$

u $0 \quad N$

c \&

2. 00

क ...

$0+40$

工

$+\pi$.

E

E $0 \approx 0$

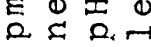

त.

वे 00

$0 \cdot r-1<$

$1 \%$

4 N

\& ${ }^{4} x^{\circ}$

+ का है .

ว 2000

$\rightarrow 0+$

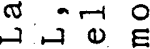

$\therefore$

되몬 
by using a chart recorder (Linseis. LS-4 series, selb, W. Germany) giving a full span of $0.1-0.5 \mathrm{pH}$ units. The experimental set up is shown in Figure 12. The ADP and ATP were prepared in the buffer being used in the experiment(see figure legends) and the $\mathrm{pH}$ was adjusted at this stage to the required level.

\subsection{FLASH-INDUCED SPECTROPHOTOMETRY}

2.7.1 Measurements of the flash-induced electrochromic bandshift

Measurements were made in a laboratory-constructed single beam spectrophotometer (Fig. 13). The chloroplast sample, contained in a $1 \mathrm{~cm}$ pathlength cuvette, was excited by a 5 us xenon flash (General Radio, stroboslave) which had been passed through a red filter (kodak, Wratten 25 and 92). The absorption change was monitored using a weak continuous measuring beam from a 150 w quartz-iodine lamp powered by a stabilised D.C. supply. The measuring beam passed through a monochromator (M380 Applied Physics Limited) giving light at $515 \mathrm{~nm}$, and after passing through the sample was detected with a photo-multiplier tube (EMF 9659B) which was protected with blue filters (Corning 4-96). The photomultiplier tube was powered by a Brandenburg 47 5R H.T. supply. The output of the photomultiplier tube was passed through a currentto-voltage converter and amplifier followed by an R.C. filtering circuit, to improve the signal to noise ratio, and stored in a $20 \mathrm{MHz}$ Datalab 922 transient recorder. The signal was then transfered to a Digital Equipment Corporation PDP-11-34 computer. 


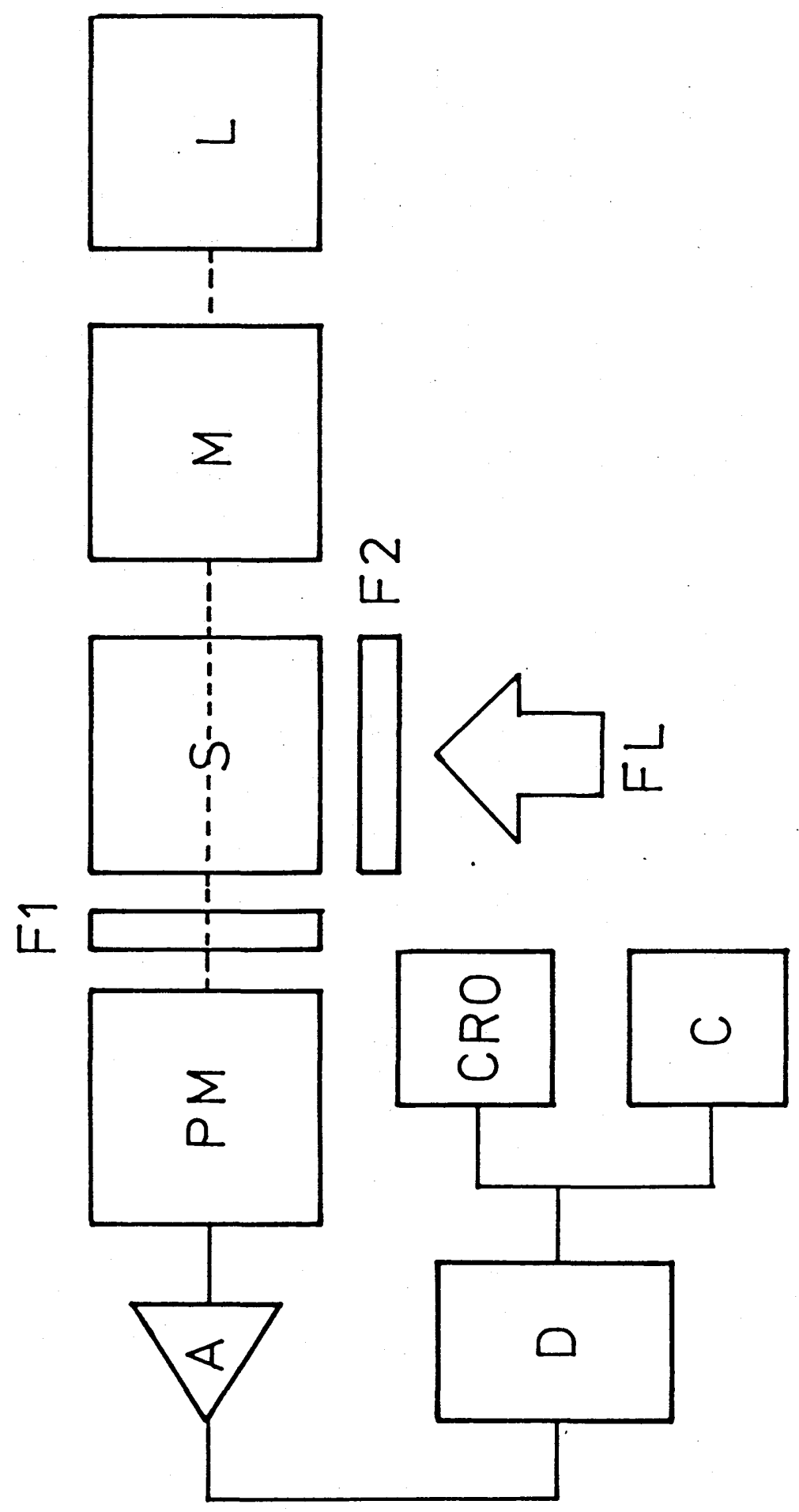

ڤั

n $\Sigma$

다 ते

ㅇ.. \&

$>00$

ज小

$\rightarrow 0$.

(1)

E 0 क

- 3 a

: न

क.

$+200$

E [1, .

○ 0 .

+..

어대

다

응

क त

ए का

(1) a

Q 20 . S n

.. \&

का 0 -

E $0 \cdot \pi 4$

बू 4

อ

(1) 0 国

步的 $E$

द

$\left.\begin{array}{r}-1 \\ v\end{array}\right), \frac{s}{0}$

- $2 \ll$

† $\mathrm{E} \ddot{\mathrm{O}}$

s. 0

证 $\sum 0$

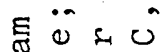

8000

of . . .

ब

त 020

o v.r 0

$0+40$

+ 工 5.4

त $E$ O

E.r 0 -

c 1000

0 250

$=$ 
The computer added a number of similar digitally converted signals together thus improving the signal-to-noise ratio: if each signal is exactly synchronised then the signal will increase as each one is summed but because the noise is random it will diminish. Unless otherwise stated the electrochromic bandshift traces shown in the figures were the summation of 64 single flash sweeps at a repetition rate of one per second. The appearance of the slow ise phase (see control Fig.24) was variable but did not affect the results of the experiments when the two types of data were compared. On the figures an arrow indicates the point at which the flash was fired.

The rate of decay of $\triangle A 515$ is indicative of the ionic permeability of the membrane. Therefore, in order to make accurate comparisons between samples, it is necessary to quantify the rate of decay. The data were fitted to a variety of functions using a computer assisted least-squares fit.

Three functions were used:

1. for a simple first-order exponential decay $Y(t)=A_{0} \exp (-k t)$

2. for a linear combination of two first-order decays : $Y(t)=A_{0} \exp (-k t)+B_{0} \cdot \exp (-1 t)$, and

3. for a curve including a second, slow phase of increase (Hipkins and Musto, 19.85) $Y(t)=\left(A_{o}+B_{o}(1-\exp -1 t)\right) \exp (-k t)$

Where

$A=$ initial amplitude

$Y=$ amplitude after a given time

$k=$ first order rate constant

$I=$ first order rate constant 


\subsubsection{Flash-induced oxonol VI absorption changes.}

The experimental arrangement used for measurements of flash-induced oxonol absorption changes was essentially the same as that described in 8.7.1 for $\Delta A 515$.

Oxonol VI was synthesised by Dr. V. Math of the Botany Department Glasgow University, adapting the method of Smith et al, (1982).

\subsection{MEASUREMENT OF FLUORESCENCE.}

\subsubsection{Chlorophylla Fluorescence.}

These measurements were made in a laboratory constructed flucrimeter (Fig. 14). The light was provided by a $150 \mathrm{w}$ quartz-iodine lamp which, was passed through a filter combination (Corning 4-76. and 4-96; Balz en Camflex infra-red filter), to give broad-band blue light. A solenoid-operated shutter was used to admit the light to the chamber. Fluorescence was measured at right-angles to the actinic beam with a photomultiplier tube (EMI:9659) powered by a Bradenburg $475 \mathrm{R}$ H.T. supply and protected with a $685 \mathrm{~nm}$ interference filter (Balzens B40) and an RG 695 (schott) cut-off filter. The photo current was passed through a current-tovoltage converter and amplifier and was passed to a potentiometric chart recorder. In the case of fast fluorescence transients the output from the amplifier was passed to a Datalab (DL 922) digital transient recorder and onto the computer. 


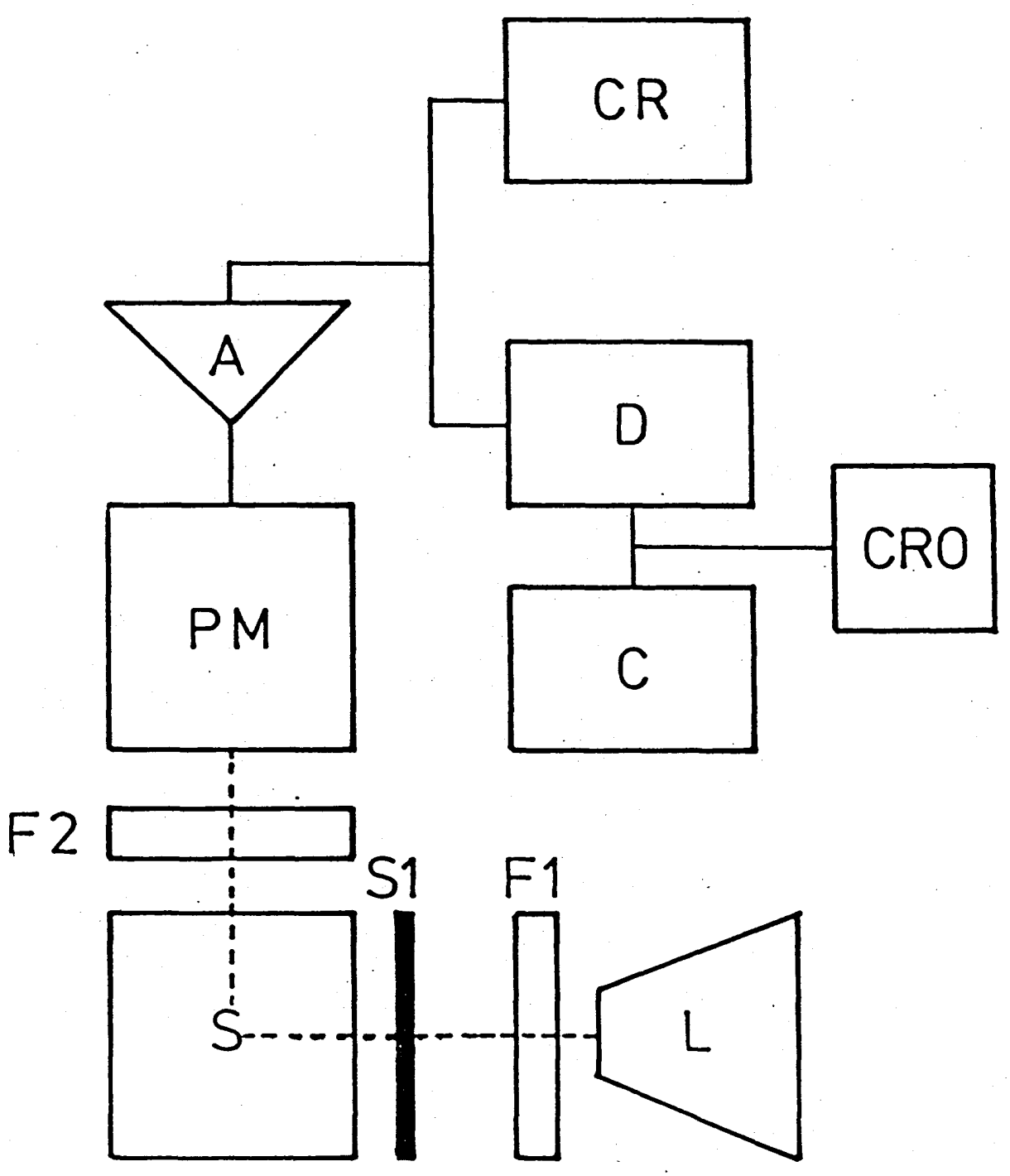

FIGURE 14 .

Schematic diagram of the fluorimeter. The symbols are: L, light source; S1, shutter; F, blue filter; $F$; red filter; P.M, photomultiplier tube; C.R., chart recorder; CRO, oscilliscope; C, computer and $A$, amplifier. 


\subsubsection{9-Aminoacridine Fluorescence (Pick and McCarty, 1980)}

Measurements were made in a laboratory-constructed

fluorimeter. The chloroplast sample, to which 9-Aminoacridine had been added, was contained in

a lcms pathlength cuvette. The 9-AA fluorescence was excited with $400 \mathrm{~nm}$ light derived from a $150 \mathrm{~W}$ quartz-iodine lamp, and passed through a monochromator. Fluorescence was detected, at right angles to the exciting light, by a photomultiplier tube (EMI 9659B)

powered bysa Bradenburg $475 \mathrm{R}$ H.T. supply and protected by a green (Wratten 57) and a blue (Corning, 4-96) filters. The output from the photomultiplier tube was passed to a current-tovoltage converter and amplifier, then to a potentiometric chart recorder (Linseis).

Photosynthesis was excited by light, from a second quartz-iodine lamp, which had been passed through a red (Wratten 70) filter (see Fig. 15).

\subsection{COUPLING FACTOR ISOLATION AND PURIFICATION.}

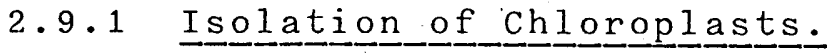

$200 \mathrm{~g}$ of peas (see 2.1) was homogenised in $250 \mathrm{ml}$ of a medium containing: $0.4 \mathrm{M}$ Sucrose, $20 \mathrm{mM}$ Tricine and $10 \mathrm{mM} \mathrm{NaCl}$. (Lien and Racker, 1971). The extraction procedure was as described in section 2.1 with the exception that the filtrate was centrifuged at $3000 \mathrm{x}$ for $10 \mathrm{~min}$. The pellet produced was washed in the extraction medium and centrifuged as before, producing 'intact' chloroplasts. 

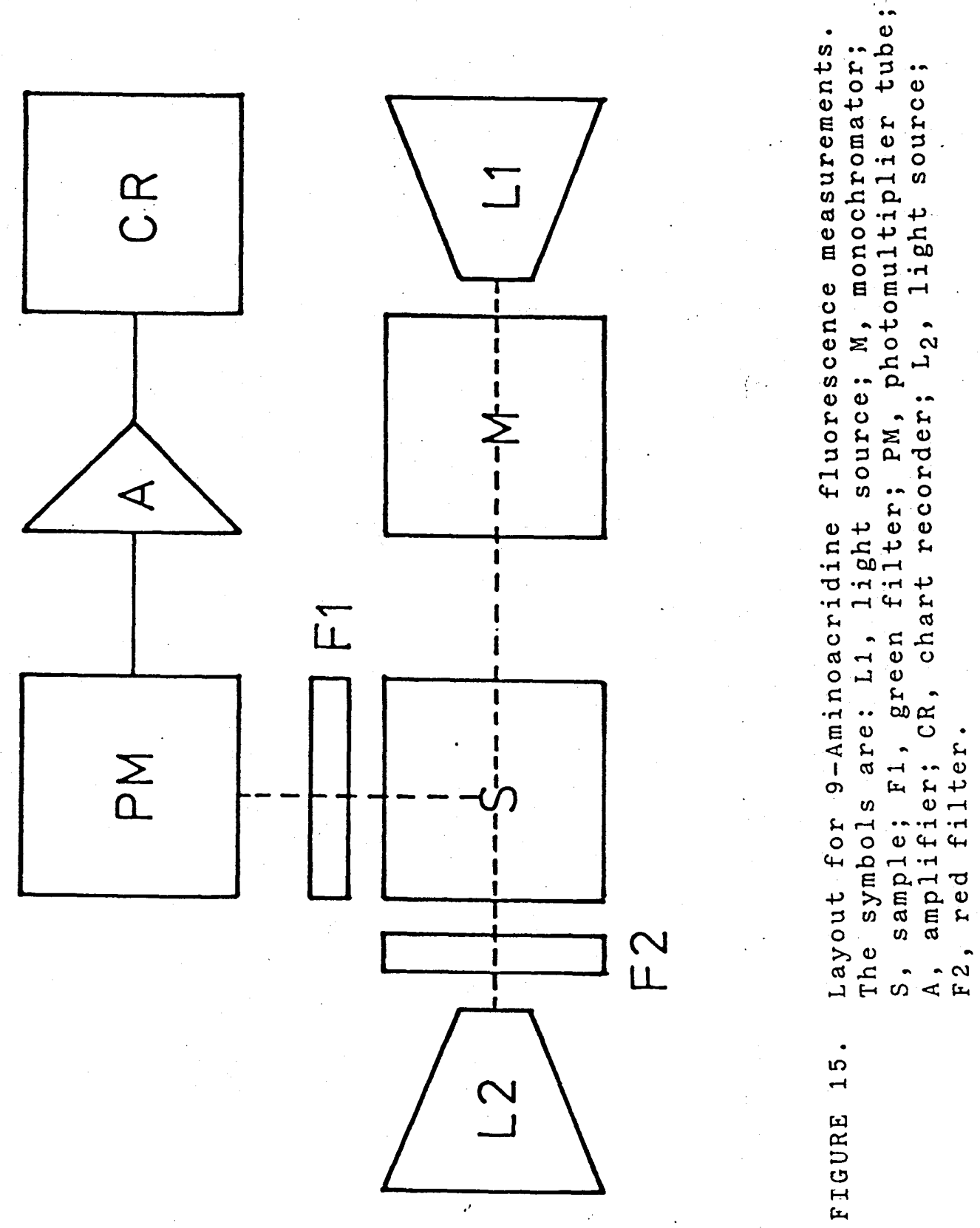


\subsubsection{Extraction of the Coupling Factor.}

The pellet from the above procedure was resuspended, in a medium containing: $10 \mathrm{mM} \mathrm{NaCl}, 2 \mathrm{mM}$ Tricine pH8.0, and was incubated on ice in the dark for 1 hour. This procedure caused osmotic lysis of the chloroplasts, producing free thylakoid membranes. The suspension was then diluted in the resuspending medium to give a chlorophyll concentration of $100 \mu \mathrm{g} \mathrm{ml}^{-1}$ and, in the case of enzyme treated samples, the required amount of trypsin or chymotrypsin was added. on completion of enzyme treatment, trypsin inhibitor was added, and the suspension centifuged (MSE high speed 18) at $10000 \mathrm{x}$ for $10 \mathrm{~min}$. The pellet was resuspended in a medium, containing $0.25 \mathrm{M}$ sucrose, $10 \mathrm{mM}$ Tris $\mathrm{SO}_{4}(\mathrm{pH} 7.6)$, 1mM EDTA, $2 \mathrm{mM}$ ATP and $5 \mathrm{mM}$ dithiothreitol, to give a chlorophyll concentration of $2.5-3 \mathrm{mg} \mathrm{ml}^{-1}$. To this solution a half-volume of chloroform was added at room temperature and the mixture was stirred for $15 \mathrm{~s}$. This was centrifuged (EBA 25 Hettich, Arnold R. Horwell Limited; London NW6 2BD) at $300 \mathrm{x} g$ for $2 \mathrm{~min}$, the top aqueous layer was removed and recentrifuged at $12000 \mathrm{x} g$ for $30 \mathrm{~min}$ at room temperature. The golden yellow supernatant thus produced contained $\mathrm{CF}_{1}$. To store this preparation a $50 \%\left(\mathrm{NH}_{4}\right)_{2} \mathrm{SO}_{4}$ precipitate was formed: $3.14 \mathrm{~g}$ of $\left(\mathrm{NH}_{4}\right)_{2} \mathrm{SO}_{4}$ was added for each $10 \mathrm{~m} 1$ of solution containing $\mathrm{CF}_{1}$, this was centrifuged at $12000 \mathrm{x}$ f for $20 \mathrm{~min}$ and the pellet was stored in a saturated solution of $\left(\mathrm{NH}_{4}\right)_{2} \mathrm{~S}_{4} \mathrm{O}_{4}$ at $4{ }^{\circ} \mathrm{C}$. (Younis et al,1977) 


\section{9 .3 Purification of $\mathrm{CF}_{1}$}

The $\left(\mathrm{NH}_{4}\right)_{2} \mathrm{SO}_{4}$ precipitate containing $\mathrm{CF}_{1}$ was resolubilised in a medium containing $20 \mathrm{mM}$ Tricine, $2 \mathrm{mM}$ EDTA and $1 \mathrm{mM}$ DTT $(\mathrm{pH} 8.0)$. This suspension ( $\sim 3 \mathrm{ml}$ ) was passed through a Sephacryl (Pharmacia) S 300 column $(100 \mathrm{~cm} \times 3 \mathrm{~cm})$ which had been washed with the buffer containing: $20 \mathrm{mM}$ Tricine, $2 \mathrm{mM}$ EDTA and $1 \mathrm{mM}$ DTT. $10 \mathrm{ml}$ fractions were collected ( 2070 ulltrorac 11, LKB Biochrom,Cambridge) and were assayed spectrophotometrically $(280 \mathrm{~nm})$ for protein. $\mathrm{CF}_{1}$ was present in fractions 10 to 15 ; these fractions were pooled and concentrated to a volume of $\sim 2 \mathrm{ml}$ using an ultrafiltration device (Amicon PM Model 12). The concentration of protein in this extract was measured using a tannin protein assay and the presence of $C_{1}$ identified by polyacrylamicie gel electrophoresis.

\section{9 .4 Polyacrylamide Gel Electrophoresiś}

Electrophoresis employed a vertical. slab-gel apparatus with a gradient gel of 12.5 to $15 \%$ (w/v) acrylamide in the presence of $0.4 \%(w / v)$ sodium do-decyl sulphate (SDS). The running gel buffer contained $1.5 \mathrm{M}$ Tris$\mathrm{HCL}, 0.4 \% \operatorname{SDS}(\mathrm{w} / \mathrm{v}) \mathrm{pH} 8.0$ and the stacking gel buffer contained $0.5 \mathrm{M}$ Tris-HCL, $0.4 \% \operatorname{SDS}(\mathrm{w} / \mathrm{v}) \mathrm{pH} 6.8$. The protein sub-units were separated using a boiling solution which contained $50 \mathrm{mM}$ Tris, $2 \% \operatorname{SDS}(\mathrm{w} / \mathrm{v}), 10 \%$ glycerol (v/v), $2 \%$ Mercaptoethanol (v/v) and bromophenol blue,pH 8.0. The gels were run at a current of $30 \mathrm{~mA}$ ( 2103 power supply LKB Biochrom) for 6 hours using an electrolyte containing $23 \mathrm{mM}$ Tris, $0.192 \mathrm{M}$ glycine and $0.1 \% \operatorname{SDS}(\mathrm{w} / \mathrm{v})$. The protein was stained overnight in a solution containing $25 \%$ propanol (v/v), $10 \%$ acetic acid (v/v) and $0.04 \%$ coomassie blue $(\mathrm{w} / \mathrm{v})$, and destained in a solution containing $10 \%$ methanol $(v / v)$ and $10 \%$ acetic acid $(i v / v)$. (Laemmli, 1970) 


\begin{tabular}{|c|c|}
\hline & THE EFFECTS OF TRYPSIN AND \\
\hline & CHYMOTRYPSIN ON ELECTRON \\
\hline & TRANSPORT IN ISOLATED CHLOROPLASTS. \\
\hline
\end{tabular}


RE SUULISS.

THE EFFECTS OF TRYPSIN AND CHYMOTRYPSIN ON ELECTRON TRANSPORT IN ISOLATED CHLOROPLASTS.

\subsection{INTRODDUCTION.}

In this chapter a comparison is made between the effects of trypsin and chymotrypsin on electron transport in isolated thylakoids. A number of workers has previously: shown that trypsin can inhibit and uncouple electron transport. (Mantai, 1969 and 1970; Selman and Bannister, 1973 Regitz and ohad, 1976; Renger, 1976). The conclusion from these papers was that electron transport was inhibited primarily by an effect on the PSII polypeptides with PSI being unaffected: the water splitting enzyme complex was proposed to be the primary site of attack (Regitz and Ohad, 1979; Selman and Bannister, 1973), but subsequent work (Renger,1976; Renger et al,1976) suggested that a proteinaceous component covering over the reducing side of PSII was responsible for the observed functional changes. Further evidence to support this proposal came from studies using the inhibitor DCMU which is known to inhibit electron transfer between the reaction centre of PSII and the Plastoquinone pool without affecting the functional integrity of the reaction centre (Bennoun, 1970). l. It was shown that digestion of membranes with trypsin. led to the removal of the DCMU binding sites and DCMU is then unable to inhibit electron transfer (Tischer and strotmann, 1979; Renger et al, 1981). The binding site is now known to be part of a $32 \mathrm{KDa}$ protein (Mullet and Arntzen, 1981) which is a functional part of PSII, lying at the outside of the membrane on the reducing side of PSII. 
Trypsin is a specific protease breaking bonds which involve arginine or lysine residues on 1y: since trypsin has the ability to decrease herbicide binding (Tischer and Strotmann, 1979; Trebst, 1979; Pfister and Arntzen,1979) it is attractive to propose that arginine and lysine are important bonds participating in the binding site. With this in mind, it was decided to use another specific protease, chymotrypsin, which has specificity for aromatic amino acids, i.e. tryptophan, tryosine and phenylalanine. It was hoped from these experiments to correlate any differences in changes in function, brought about by these enzymes, with their different specificities. The results in this section show that both trypsin and chymotrypsin havesimilar effects on electron transport but some subtle differences were evident.

\section{3 . 2 RESULTS.}

Figures 16 and 17 show that when thylakoid membranes were incubated in the presence of trypsin the rate of oxygen evolution became sensitive to the nature of the electron acceptor present. When potassium ferricyanide $\left(\mathrm{K}_{3} \mathrm{Fe}(\mathrm{CN})_{6}\right)$ was used as an electron acceptor the rate of oxygen evolution was little affected by trypsin incubation. However, if $p-b e n z o q u i n o n e$ was used almost total inhibition of oxygen evolution was observed (Figs 16 and 17). These results are in agreement with those of Renger et al (1976). Chymotrypsin (Figs 18 and 19) displayed similar but less marked effects as trypsin: in this case inhibition of oxygen evolution occurred with both $\mathrm{K}_{3} \mathrm{Fe}(\mathrm{CN})_{6}$ or $\mathrm{p}$-benzoquinone, but the reduction in the rate of oxygen evolved was greater in the presence of p-benzoquinone. These results suggested 


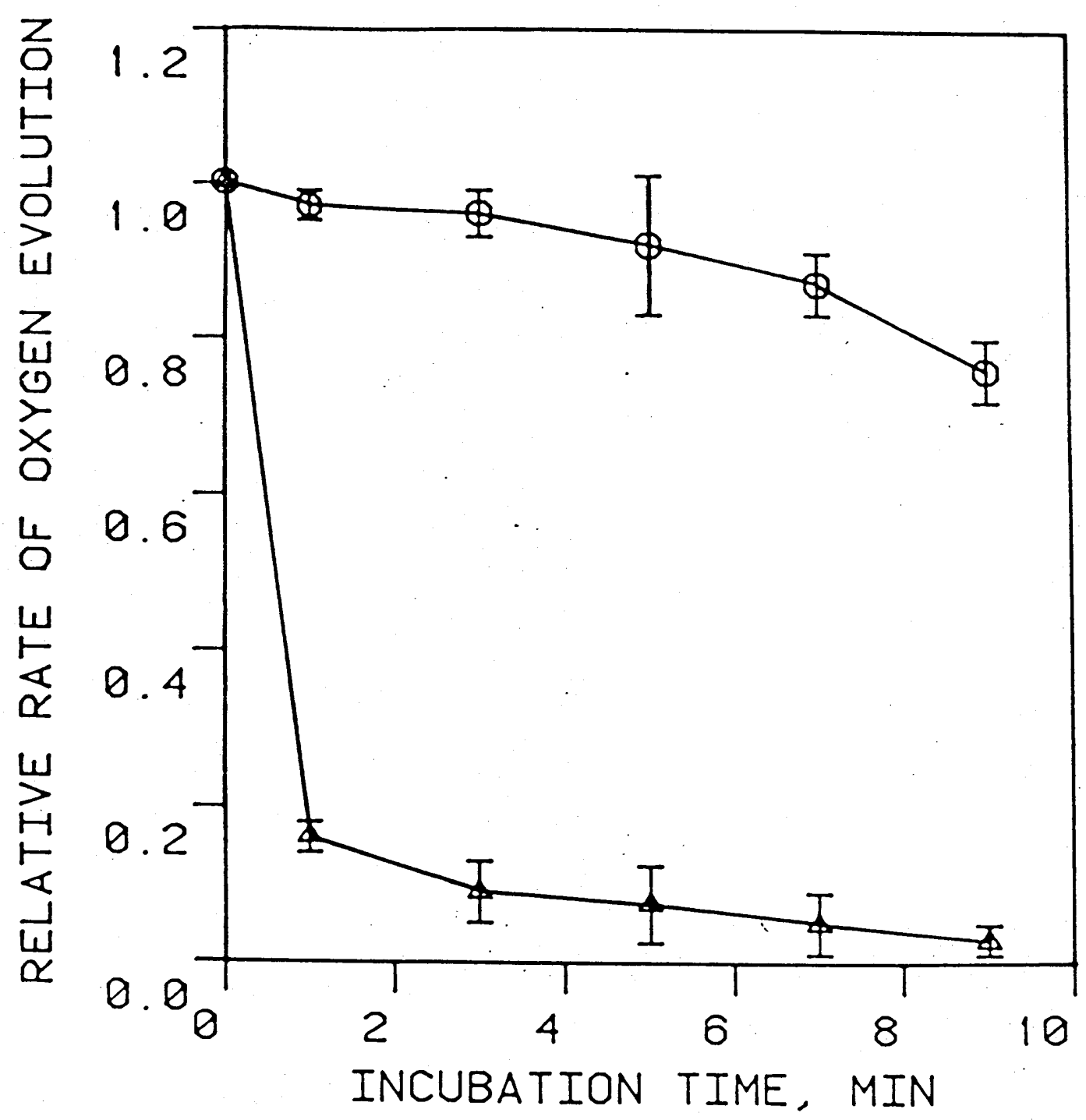

FIGURE 16 .

The effect of trypsin on the rate of oxygen evolution in the presence of $\mathrm{K}_{3} \mathrm{Fe}(\mathrm{CN})(\mathrm{O})$ or p-Benzoquinore $(\Delta)$, in uncoutpled thylakoid membranes. Thylakoids with a chlorophyll concentration of $50 \mu g m 1^{-1}$ were incubated with trypsin $(50 \mu g m 1 \stackrel{-1}{-1})$ in $2 \mathrm{ml}$ of medium containing: Tricine $(20 \mathrm{mM}), " \mathrm{KCl}(10 \mathrm{mM})$ and $\mathrm{MgCl}_{2}(2 \mathrm{mM}) \mathrm{pH} 7.4$. The reaction was stopped by adding trypsin inhibitor $(1 \mathrm{mgm} 1-1)$. All samples contained $\mathrm{NH}_{4} \mathrm{Cl}$ $(2.5 \mathrm{mM})$ and either $\mathrm{K}_{3} \mathrm{Fe}(\mathrm{CN})_{6}(1.5 \mathrm{mM})$ or $\mathrm{p}$-benzoquinone ( $3 \mathrm{mM})$.

The control rate of oxygen evolution was usually:

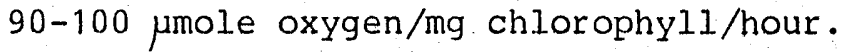




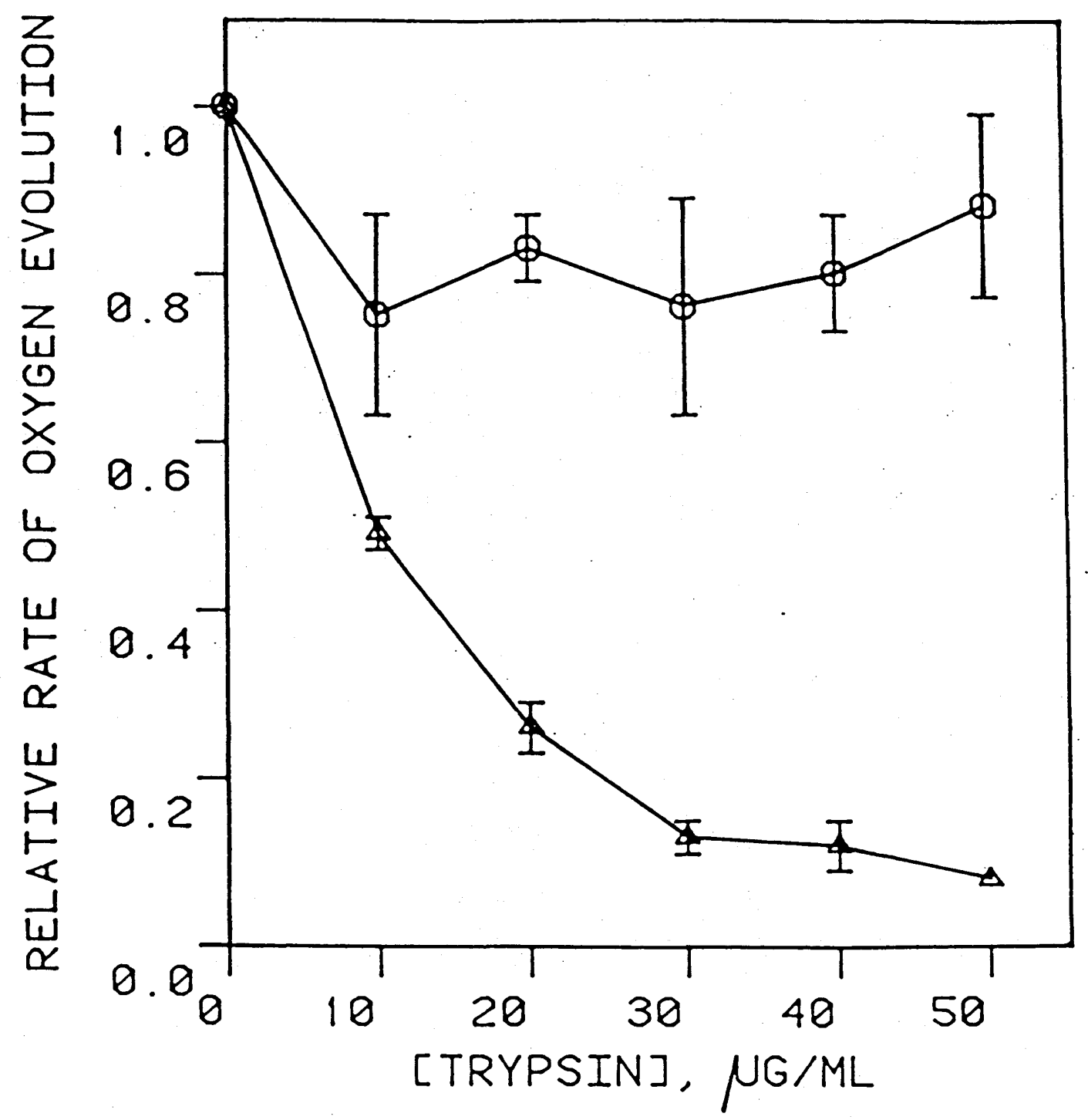

FIGURE 17 .

The effect of trypsin or the rate of oxygen evolution, in the presence of $\mathrm{K}_{3} \mathrm{Fe}(\mathrm{CN})_{6}(\mathrm{O})$ or p-benzoquinone $(\Delta)$, in uncoupled thylakoid membranes. Thylakoids with a chlorophyll concentration of $50 \mu \mathrm{gmi}^{-1}$ were incubated with trypsin ( $3 \mathrm{~min}$ ) in $2 \mathrm{ml}$ of medium containing: Tricine $(20 \mathrm{mM}), \mathrm{KCl}(10 \mathrm{mM})$ and $\mathrm{MgCl}_{2}$ ( $\left.2 \mathrm{mM}\right) \mathrm{pH} .7 .4$, The reaction was stopped by adding trypsin inhibitor $(1 \mathrm{mgm1}-1)$. A.11 samples contained $\mathrm{NH}_{4} \mathrm{Cl}$ $(2.5 \mathrm{mM})$ and either $\mathrm{K}_{3} \mathrm{Fe}(\mathrm{CN})_{6}(1.5 \mathrm{mM})$ or $\mathrm{p}$-benzoquinone $(3 \mathrm{mM})$. 


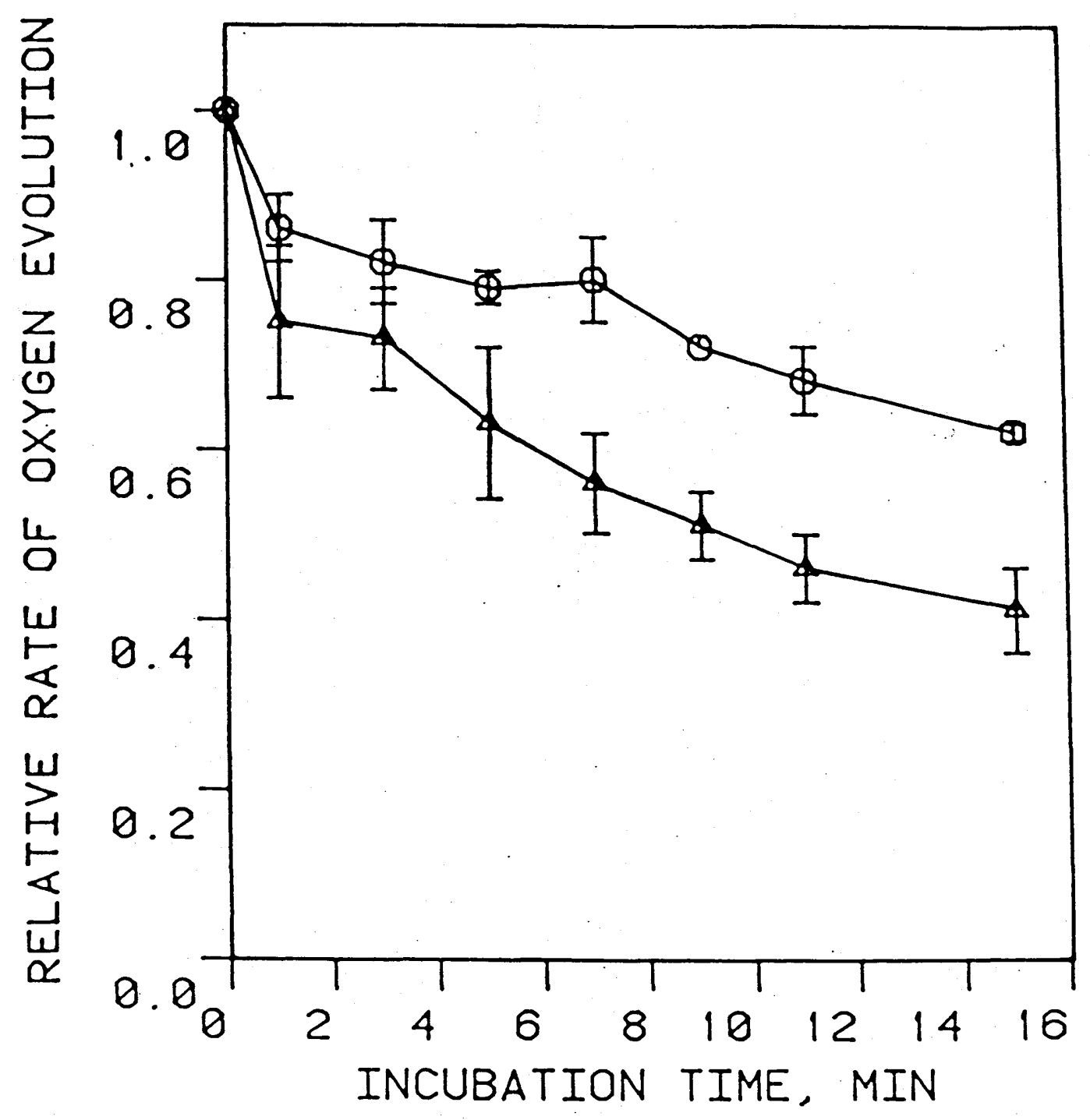

FIGURF, 18 .

The effect of chymotrypsin on the rate of oxygen evolution in the presence of $\mathrm{K}_{3} \mathrm{Fe}(\mathrm{CN})_{6}$ $(0)$ or p-benzoquinone $(\Delta)$, in uncoupled thylakoid membranes. Thylakoids with a chlorophyll concentration of $50 \mu^{-1}$ were incubated with chymotrynsin ( $\left.100 \mathrm{Mgml}^{-1}\right)$ in $2 \mathrm{ml}$ of medium containing: Tricine ( $20 \mathrm{mM})$, $\mathrm{KCl}(10 \mathrm{mM})$ and $\mathrm{MgCl}_{2}$ (2 $\left.\mathrm{mM}\right) \mathrm{pH} 7.4$. The reaction was stopped by adding trypsin inhibitor $\left(1 \mathrm{mgmi}^{-1}\right)$. All samples contained $\mathrm{NH}_{4} \mathrm{Cl}(2.5 \mathrm{mM})$ and either $\mathrm{K}_{3} \mathrm{Fe}(\mathrm{CN})_{6}(1.5 \mathrm{mM})$ or p-benzoquinone ( $3 \mathrm{mM})$. 


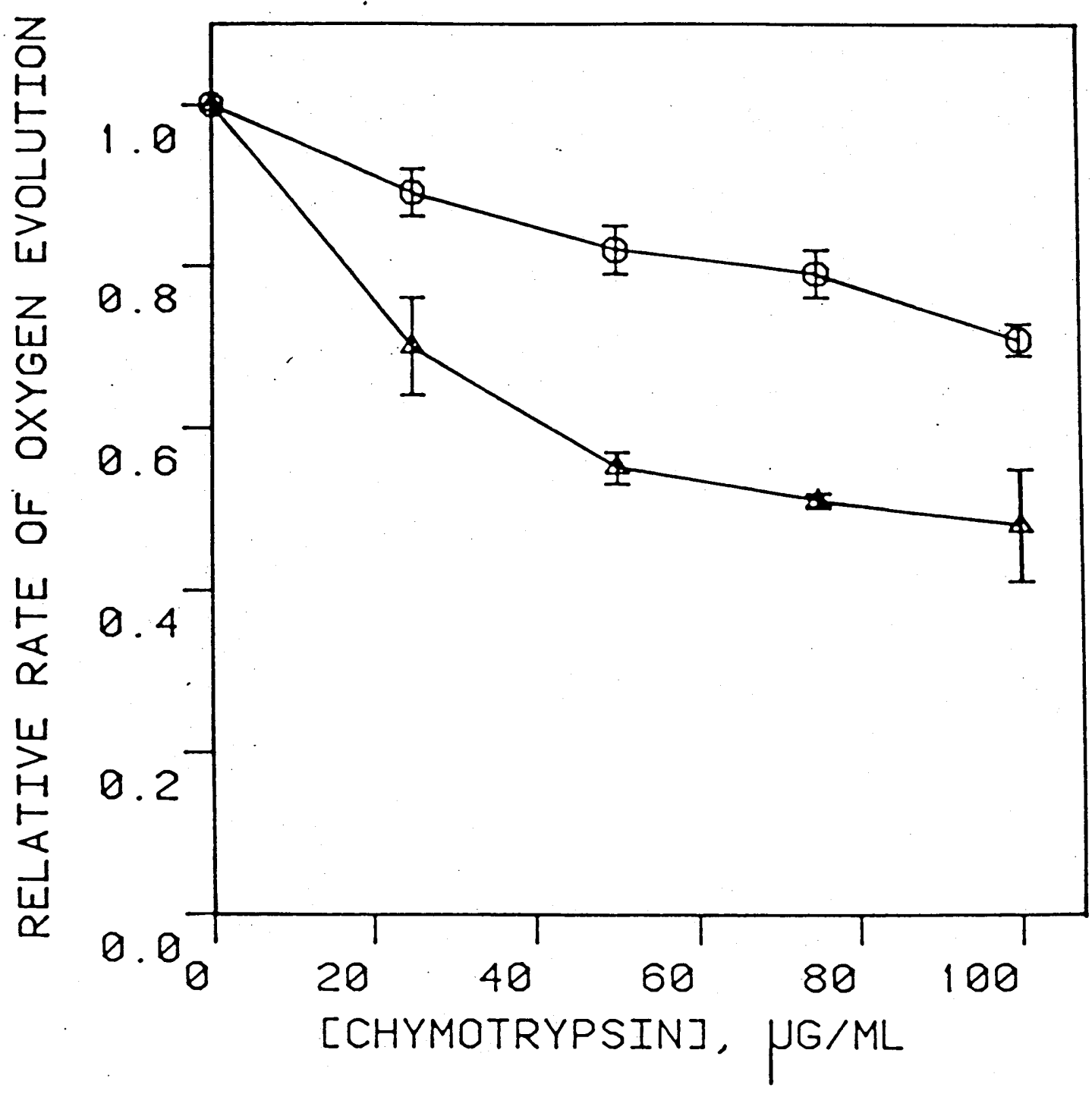

FIGURE 19 .

The effect of chymotrypsin on the rate of oxygen evolution, in the presence of $\mathrm{K}_{3} \mathrm{Fe}(\mathrm{CN})_{6}$ $(0)$ or $p$-benzoquinone $(\Delta)$, in uncoupled thylakoid membranes. Thylakoids with a chlorophyll concentration of $50 \mu^{-1 m l^{-1}}$ were incubated with chymotrypsin ( $5 \mathrm{~min})$ in $2 \mathrm{ml}$ of medium containing: Tricine $(20 \mathrm{mM})$, $\mathrm{KCl}(10 \mathrm{mM})$ and $\mathrm{MgC} \mathrm{I}_{2}(2 \mathrm{mM}) \mathrm{pH} 7.4$. The reaction was stopped by adding trypsin inhibitor $\left(1 \mathrm{mgmi} 1^{-1}\right)$. All samples contained $\mathrm{NH}_{4} \mathrm{Cl}$ $(2.5 \mathrm{mM})$ and ei ther $\mathrm{K}_{3} \mathrm{Fe}(\mathrm{CN})_{6}(1.5 \mathrm{mM})$ or $\mathrm{p}$ - benzoquinone ( $3 \mathrm{mM})$. 
that chymotrypsin may have the ability partially to digest a PSII protein necessary to mediate electron transport from Qa, the primary acceptor of PSII, to $Q b$ which acts as a two-electron gate between $Q$ and the plastoquinane pool $(1.4 .3)$. To test this proposal further the effect of trypsin and chymotrypsin on the inhibitory action of DCMU was investigated; the results are shown in Figures 20 and 21. Trypsin was shown (Fig. 20) to induce DCMU insenstive oxygen evolution, thus supporting the results of Renger et al (1976) and pfister et al (1979). Figure 21 shows that after chymotrypsin incubation DCMU is unable totally to inhibit oxygen evolution as can be seen in the case of the controls. This result again suggests that chymotrypsin was able to digest the PSII protein in a manner similar to trypsin, in spite of their different specificities.

The experiments previously described in this section concerned thylakoids which had been uncoupled, using $\mathrm{NH}_{4} \mathrm{Cl}$, prior to measurements being made. The effects of trypsin and chymotrypsin on coupled electron transport are shown in Figures 22 and 23; the enzyme concentrations used here were an order of magnitude lower than in the experiments described previously. Under these conditions it can be seen that trypsin Figure 22 stimulated the rate of $\mathrm{O}_{2}$ evolution in both dark-and light - treated samples. At incubation times less than $5 \mathrm{~min}$ there was a significant difference between dark-and light-treated samples. However, after lomin incubation both treatments gave a similar level of oxygen evolution. The maximum rate of oxygen evolution induced by trypsin was almost that observed with $\mathrm{NH}_{4} \mathrm{Cl}$. This uncouling effect is most likely due to.digestion of the CF lMoroney and McCarty, 1982), the extrinsic portion of the ATPase 


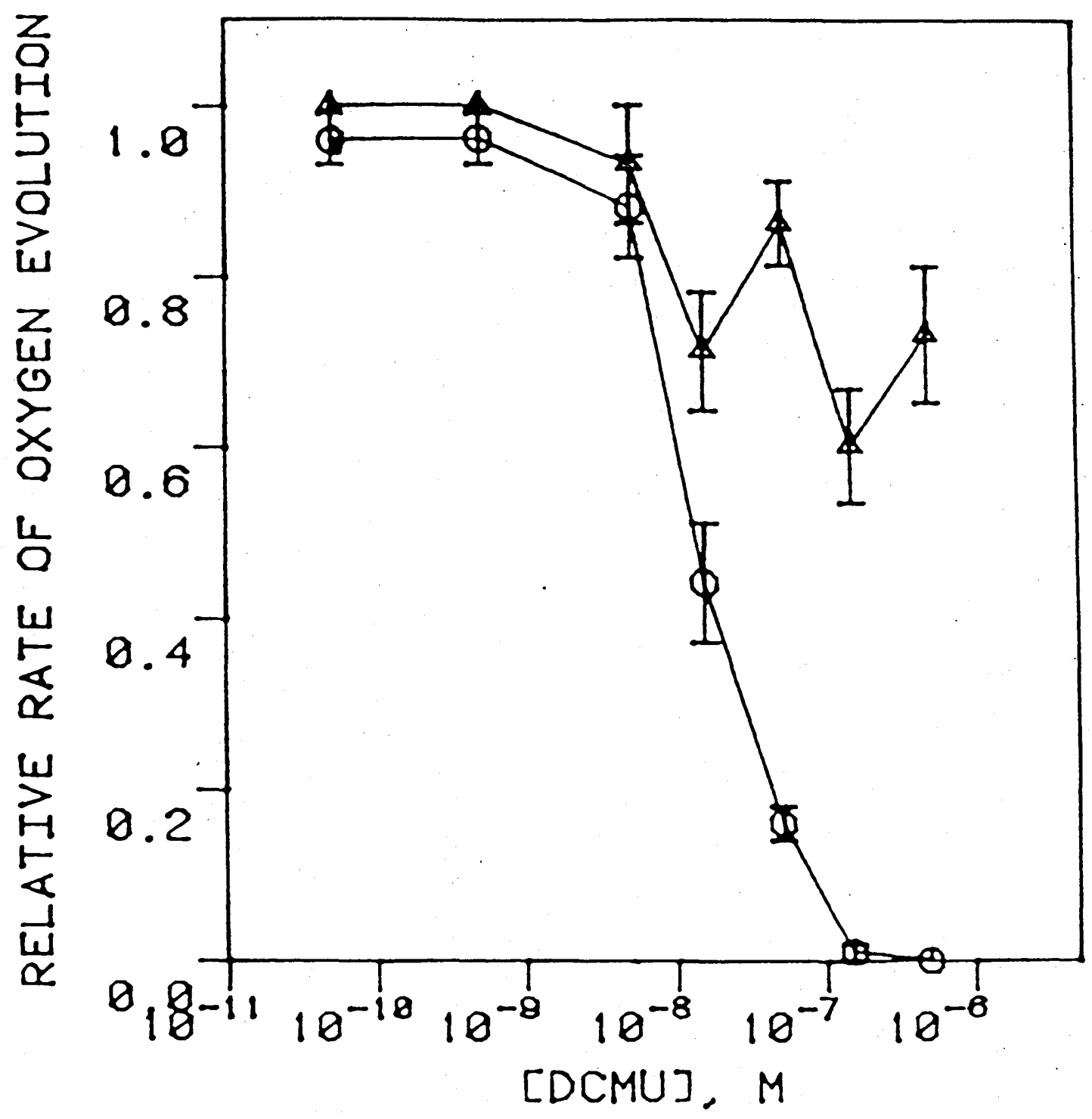

FIGURE 20 .

The effect of trypsin on the inhibition of oxygen evolution by DCMU on uncoupled thylakoid membranes. Control ( 0 ), trypsin $(\Delta)$. Thylakoids with a chlorophyll concentration of $50 \mu g m 1^{-1}$ were incubated with trypsin $(50 \mu \mathrm{gml}-1)$ for $8 \mathrm{~min}$ in $2 \mathrm{ml}$ of medium containing Tricine $(20 \mathrm{mM}), \mathrm{KCl}$ $(10 \mathrm{mM})$ and $\mathrm{MgCl}_{2}(2 \mathrm{mM}) \mathrm{pH} \mathrm{7.4}$. The reaction was stopped by adding trypsin inhibitor $\left(1 \mathrm{mgml}^{-1}\right)$. All samples contained $\mathrm{K}_{3} \mathrm{Fe}(\mathrm{CN})_{6}(1.5 \mathrm{mM})$ and $\mathrm{NH}_{4} \mathrm{Cl}$ $(2.5 \mathrm{mM})$. 


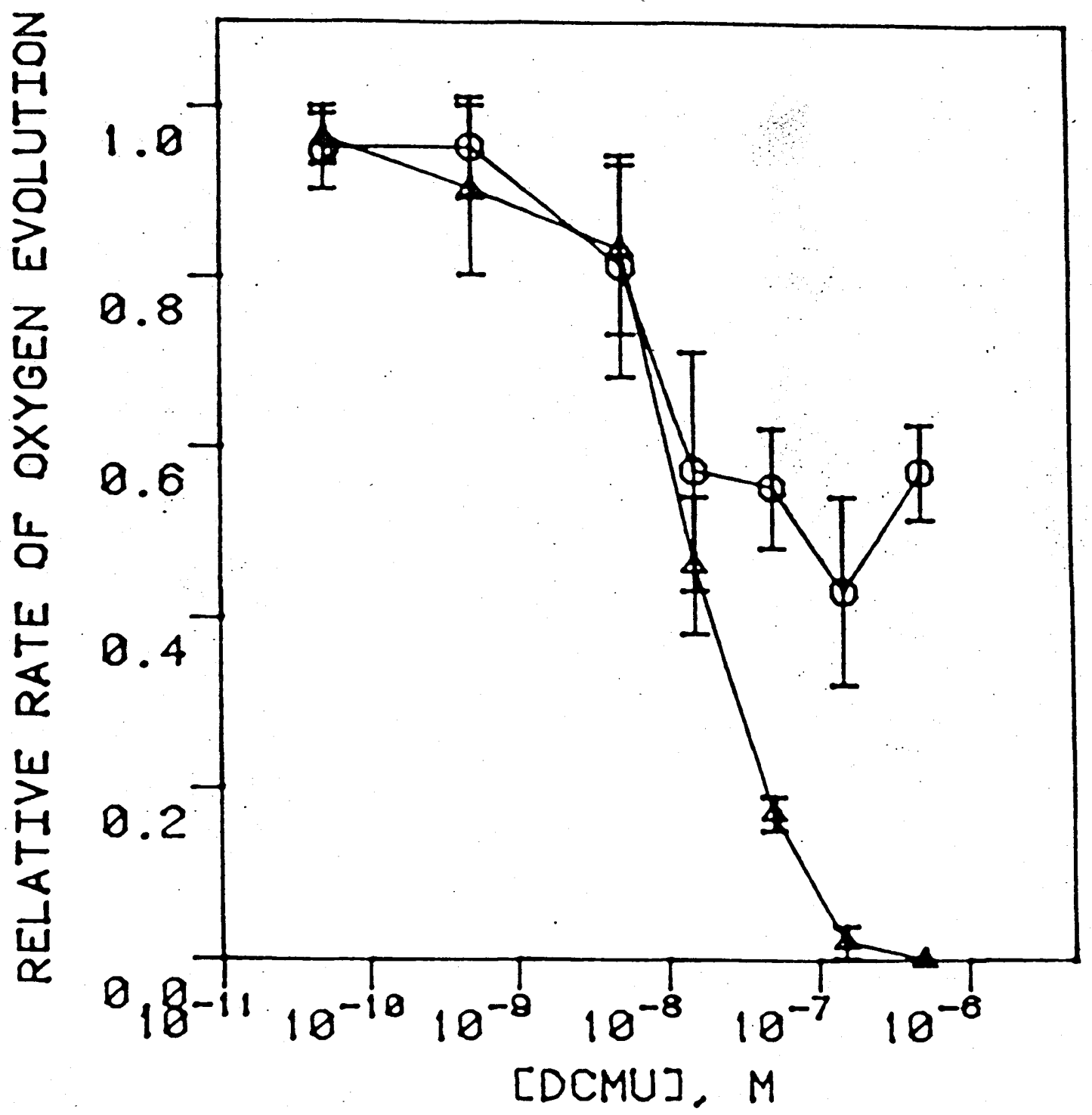

FIGURE 21 .

The effect of chymotrypsin on the inhibition of oxygen evolution of DCMU. on uncoupled thylakoid membranes. Control $(\Delta)$, trypsin (O). Thylakoids with a chlorophyl1 concentration of $50 \mu \mathrm{gml}^{-1}$ were incubated with chymotrypsin. $\left(100 \mu \mathrm{gm} \mathrm{1}^{-1}\right)$ for $5 \mathrm{~min}$ in $2 \mathrm{ml}$ of medium containing Tricine $(20 \mathrm{mM})$, $\mathrm{KCl}(10 \mathrm{mM})$ and $\mathrm{MgCl}_{2}(2 \mathrm{mM}) \mathrm{pH} 7.4$. The reaction was stopped by adding trypsin inhibitor $(1 \mathrm{mgmi}-1)$. All samples contained $\mathrm{K}_{3} \mathrm{Fe}(\mathrm{CN})_{6}(1.5 \mathrm{mM})$ and $\mathrm{NH}_{4} \mathrm{Cl}(2.5 \mathrm{mM})$. 


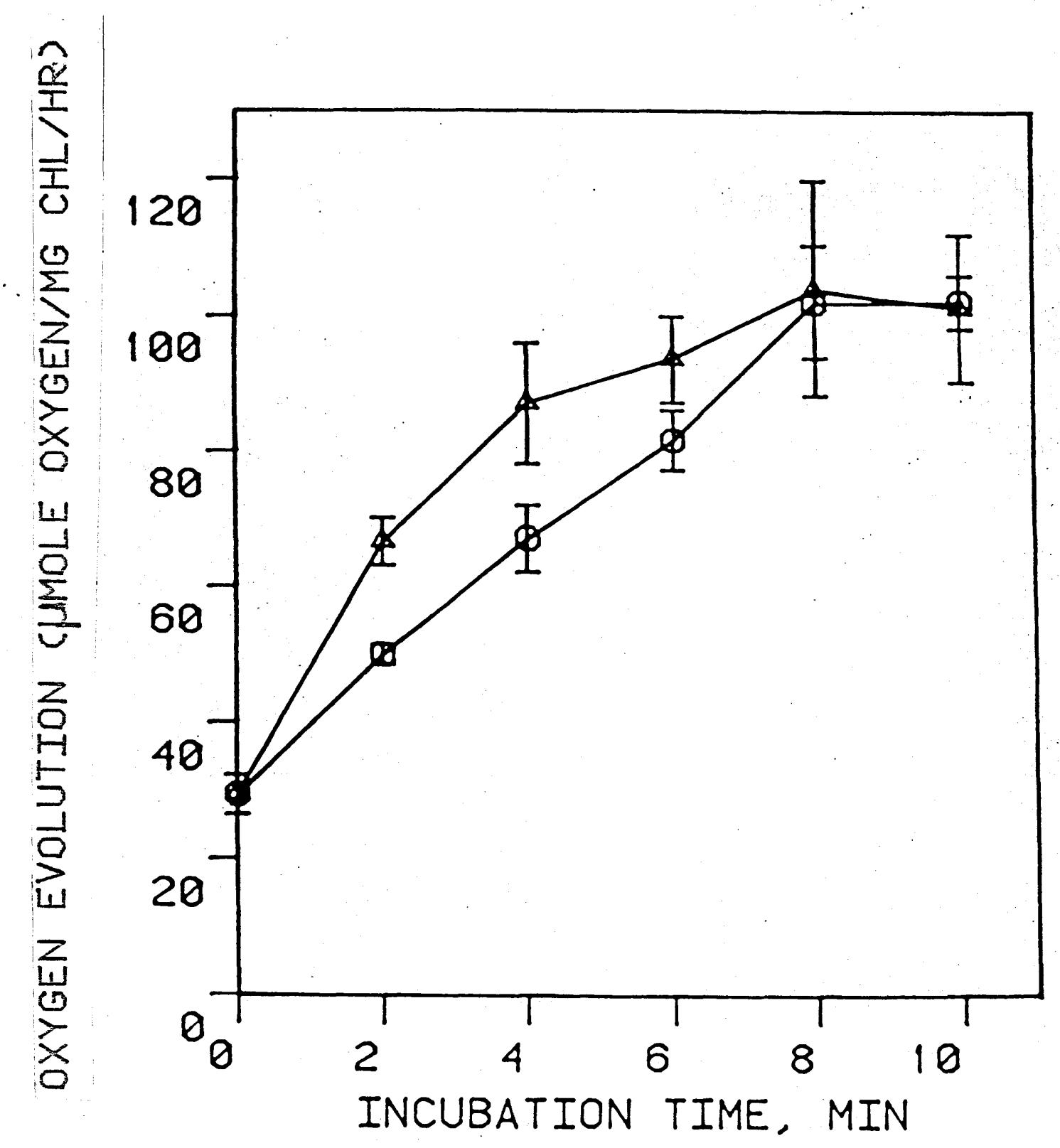

FIGURE 22 .

The effect of trypsin on the rate of oxygen evolution in coupled thylakoids. Thylakoids with a chlorophyll concentration of $50 \mathrm{Mgml}^{-1}$ were incubated with trypsin $\left(5 \mu \mathrm{gmi}^{-1}\right)$, in the light $(\Delta)$ or dark $(0)$, in $2 \mathrm{ml}$ medium containing Tricine $(20 \mathrm{mM}), \mathrm{KCl}(10 \mathrm{mM})$ and $\mathrm{MgCl}_{2}(2 \mathrm{mM}) \mathrm{pH} 7.4$. The reaction was stopped by adding trypsin inhibitor (1 $\left.\mathrm{mgml}^{-1}\right)$. $\mathrm{K}_{3} \mathrm{Fe}(\mathrm{CN})_{6}(1.5 \mathrm{mM})$ was added to all samples. 


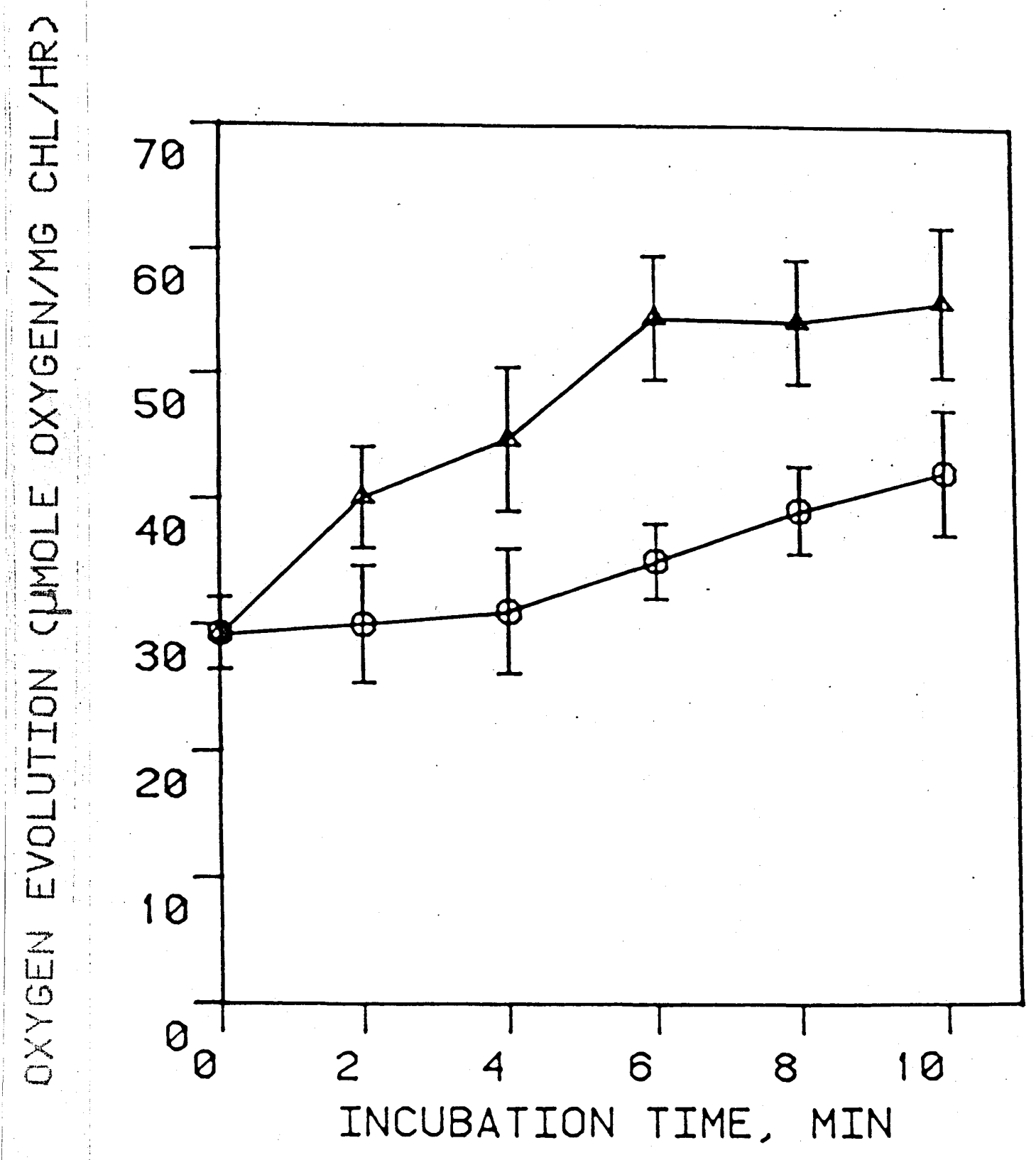

FIGURE 23 .

The effect of chymotrypsin on the rate of oxygen evolution in coupled thylakoids. Thylakoids with a chlorophyll concentration of $50 \mathrm{Mgml}^{-1}$ were incubated with chymotrypsin (i5 $\left.\mu g m 1^{-1}\right)$, in the light $(\Delta)$ or dark $(0)$, in $2 \mathrm{ml}$ medium containing Tricine $(2.0 \mathrm{mM})$, $\mathrm{KCl}(10 \mathrm{mM})$ and $\mathrm{MgCl}_{2}(2 \mathrm{mM}) \mathrm{pH} 7.4$, The reaction was stopped by adding trypsin inhibitor $\left(1 \mathrm{mgml}^{-1}\right) . \mathrm{K}_{3} \mathrm{Fe}(\mathrm{CN})_{6}(1.5 \mathrm{mM})$ was added to all samples. 
enzyme complex resulting in inhibition of phosphorylation (selman and Bannister, 1973). Figure 23 shows that chymotrypsin treatment of coupled thylakoids resulted in an increased rate of $\mathrm{O}_{2}$ evolution when membranes were incubated in the light. In dark-treated samples a significant stimulation of $\mathrm{O}_{2}$ evolution was seen only after $6 \mathrm{~min}$ incubation time. This result suggests that chymotrypsin may also digest partially $\mathrm{CF}_{1}$ but either (1) to a lesser extent or (2) at a different site from trypsin.

\subsection{DISCUSSION.}

3.3.1 The results in this chapter show that trypsin and chymotrypsin have similar effects on the functions of the thylakoid membrane. Both enzymes were shown to: 1) cause electron transport to be dependent on the presence of a particular electron acceptor; 2) reduce the inhibitory effect of DCMU on electron transport. These results indicate that chymotrypsin is capable of digesting the $32 \mathrm{KDa}$ protein on the acceptor side of PSII which has previously been shown to be one of the sites of attack for trypsin (Renger et al, 1976. Mullet and Artzen, 1981) The effect of chymotrypsin is less marked than that of trypsin and this may be related to their different specificities. Lysine-specific protease has been shown to impair the inhibitory effect of a DCMU- like inhibitor (SN 58132) while arginine-specific protease has no effect (Renger et al, 1983).

Renger et al $(1983)$ concluded that the lysine specific protease induces structural changes in the $32 \mathrm{KDa}$ protein (X 320-B-apoprotein) of PSII which bring about allosteric effects preventing DCMU binding. 
The results of the present experiments using chymotrypsin further support this suggestion: chymotrypsin attacks completely different bonds from tryspin yet it can bring about similar changes in function. The results in this section do not clarify whether chymotrypsin actually removes inhibitor binding sites or whether it allows electrons to pass directly from $Q$ of PSII to an external electron acceptor thus by-passing the site of DCMU inhibition.

3.3.2 To investigate the effects of trypsin and chymotrypsin on coupled thylakoid membranes, the enzyme concentration was one order of magnitude lower than that used in the previous sections. Under these conditions a difference was noted between the effects of trypsin and chymotrypsin on electron transport: trypsin stimulated electron transport significantly in both 1 ight-and dark-treated samples; chymotrypsin on the other hand had a much less marked effect than trypsin under both experimental conditions, and in the case of the dark treated samples almost no stimulation was in evidence. This effect may be due to an uncoupling of electron transport from phosphorylation induced by changes in protein structure: 1) general membrane disruption resulting in greater membrane permeability; 2) alteration of the ATPase complex either by a) complete removal of the extrinsic portion $\mathrm{CF}_{1}$ or by b) subtle changes of its structure causing an increase in membrane conductivity.

The effects of these enzymes on the conductance of the membranes and on the functions of the ATPase complex are further consideredin chapter 4. 


$\begin{aligned} \text { CHAPTER FOUR - } & \text { THE_EFFECTS_OE_TRYPSIN_AND } \\ & \text { CHYMOTRYPSIN_ON_THE_FUNCTION } \\ & \text { OF_THE_ATPaSE_ENZYME_COMPLEX. }\end{aligned}$


THE EFFECTS OF TRYPSIN AND CHYMOTRYPSIN ON THE FUNCTIONS OF THE ATPASE ENZYME COMPLEX.

\subsection{INTRODUCTION.}

In chapter 3 it was shown that incubation with trypsin, and to a lesser extent chymotrypsin, can stimulate the rate of oxygen evolution in coupled thylakoids. This effect may be due to the uncoupling of electron transport from phosphorylation, as was previously shown for trypsin (Mantai, 1970 and Selman et a 1,1973 ), possibly induced by enzymic digestion of $\mathrm{CF}_{1}$ of the ATPase enzyme complex. on the other hand the integrity of the membrane may be altered by enizyme digestion of other membrane polypeptides $(1.6)$ inducing conformational changes which result in altered membrane permeability. Previous investigations using trypsin suggest that general membrane disruption does not occur and that $\mathrm{CF}_{1}$ is not removed from the membrane (Selman et al 1973). This indicates that proteolysis is having a more subtle effect on the membrane. In support of this Moroney and McCarty (1982) showed that the permeability of the membrane could be increased by digestion of the Ysubunit of $\mathrm{CF}_{1}$ alone.

In order to investigate the effect of trypsin and chymotrypsin on membrane permeability, measurements of the electrochromic bandshift at $515 \mathrm{~nm}$ were made (see section 1.9.2). In addition, the effects of these enzymes on the coupling factor were assessed by measuring ATP hydrolysis and phosphorylation rates. 


\subsection{RESULTS.}

4.2.1 The effects of dark adaption and light incubation on the electrocchromic bandshift.

The electrochromic bandshift ( $\Delta$ A515) signal seen in dark-adapted isolated thylakoid membranes was different from that observed after the membranes had been subjected to light treatment (Fig. 24). In samples which had been dark adapted for a minimum of one hour, a characteristic electrochromic bandshift signal was obtained: a fast rise phase too rapid to be resolved by the apparatus, (probably in the ns time range, see section 1.9.1.1) followed by a slow rise phase in the ms time range; both of which decay slowly in the ms to $s$ time range (Fig.24A). When dark-adapted samples were illuminated with strong white light (120W. $\mathrm{m}^{-2}$ ) the signal changed (Fig. 24B-E). As the duration of light treatment was increased a decrease in the extent of the slow phase together with a decrease in the half-time of decay $\left(t_{1 / 2}\right)$ were observed. These modifications induced by light could be due to changes in membrane permeability induced by 1 ight energjisation of the membrane. These results indicated that it was necessary to standardise the light-pretreatment of the samples. Thus in all experiments involving measurements of the electrochromic bandshift the chloroplasts were dark adapted for at least one hour prior to making measurements. The light treatments given are noted in the experimental conditions in the figure legends.

Note:an alternative explanation for the effects of light on the electrochromic bandshift signal may be due to photo-inhibition causing an inhibitory effect on FSII. The fact that this light effect was irreversible suggests that it was not due to membrane energisation. 


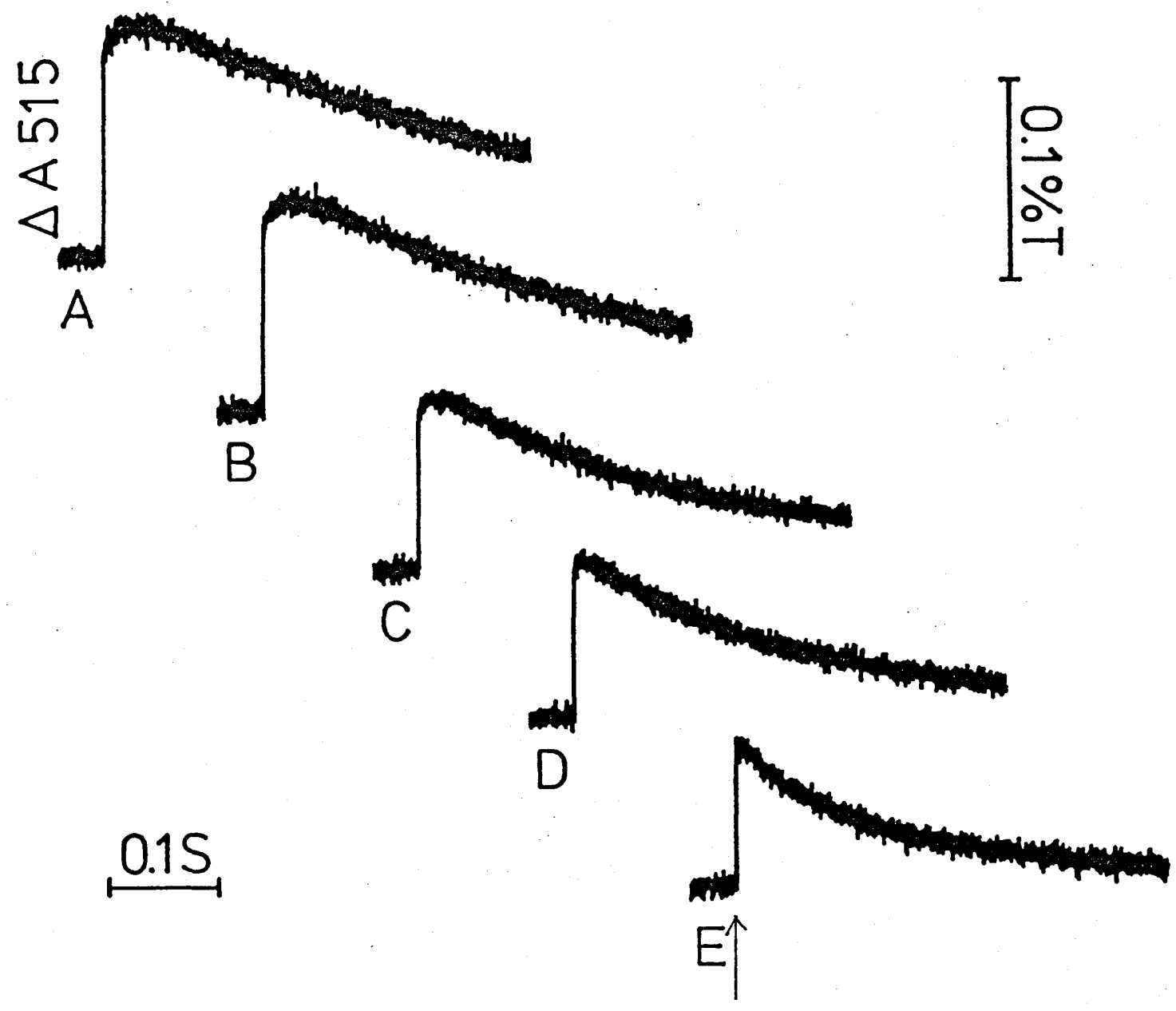

FIGURE 24 .

The effect of light $\left(120 \mathrm{wm}^{-2}\right)$ incubation of dark-adapted thylakoids on the electrochromic bandshift. Thylakoids with a chlorophyll concentration of $25 \mathrm{Mgml}^{-1}$ were incubated in $3.5 \mathrm{ml}$ of medium containing Tricine $(20 \mathrm{mM}), \mathrm{KCl}(10 \mathrm{mM})$ and $\mathrm{MgCl}_{2}(2 \mathrm{mM})$ $\mathrm{pH} 7.4$. $\mathrm{K}_{3} \mathrm{Fe}(\mathrm{CN})_{6}(0.75 \mathrm{mM})$ was added as electron acceptor. DARK: A) Control; LIGHT: B) $1 \mathrm{~min}$; C) $3 \mathrm{~min}$; D) $5 \mathrm{~min}$; E) $10 \mathrm{~min}$. 
4.2.2 Changes in the electrochromic bandshift induced by treatment of thylakoids with trypsin or chymotrypsin.

1. Incubation with trypsin or chymotrypsin in the dark. Incubation of dark-adapted thylakoids in the presence of trypsin had two effects on the electrochromic bandshift ( $\Delta$ A515): 1) the half-time decay was decreased and 2) the extent was reduced considerably (Fig. 25). The curve fitting data for these transients is shown in Table 1: it can be seen that in the control (Fig. 25(A) and Table 1(A)) there was a slow rise phase with a half rise time of $8.7 \pm 1.7 \mathrm{~ms}$ and a half-time of decay of $290 \pm 5 \mathrm{~ms}$. However, after trypsin treatment the slow rise phase was no longer evident and the decay phase was biphasic (Fig. 25 (B) and Table 1 (B)), having a slow half-time of decay of $260.5 \pm 37 \mathrm{~ms}$ and a fast halftime of decay of $38.1 \pm 8.7 \mathrm{~ms}$, these being 64 and $36 \%$ of the total signal respectively. After $10 \mathrm{~min}$ trypsin treatment (Fig. 25 (D) and Table 1 (D)) only one phase of decay was evident which had a decay half-time of $30.3 \pm 5.0 \mathrm{~ms}$. The extent of the bandshift was also affected and, after $10 \mathrm{~min}$ trypsin incubation, only $30 \%$ of the extent remained as compared to the control (Table 1). The effect of chymotrypsin on dark-adapted thylakoid membranes is shown in Figure 26 and the curve fitting data for these transients is shown in Table 2. The data shows that the control (Fig. 26 (A) and Table $2(A))$ had a slow rise phase with a half-time of 11.7 $\pm 2 \mathrm{~ms}$ and a half-time decay rate of $280.6 \pm 5 \mathrm{~ms}$. After treatment with chymotrypsin for $2 \mathrm{~min}$ the slow rise phase was no longer evident and the decay was biphasic having a slow phase with a half-time decay 

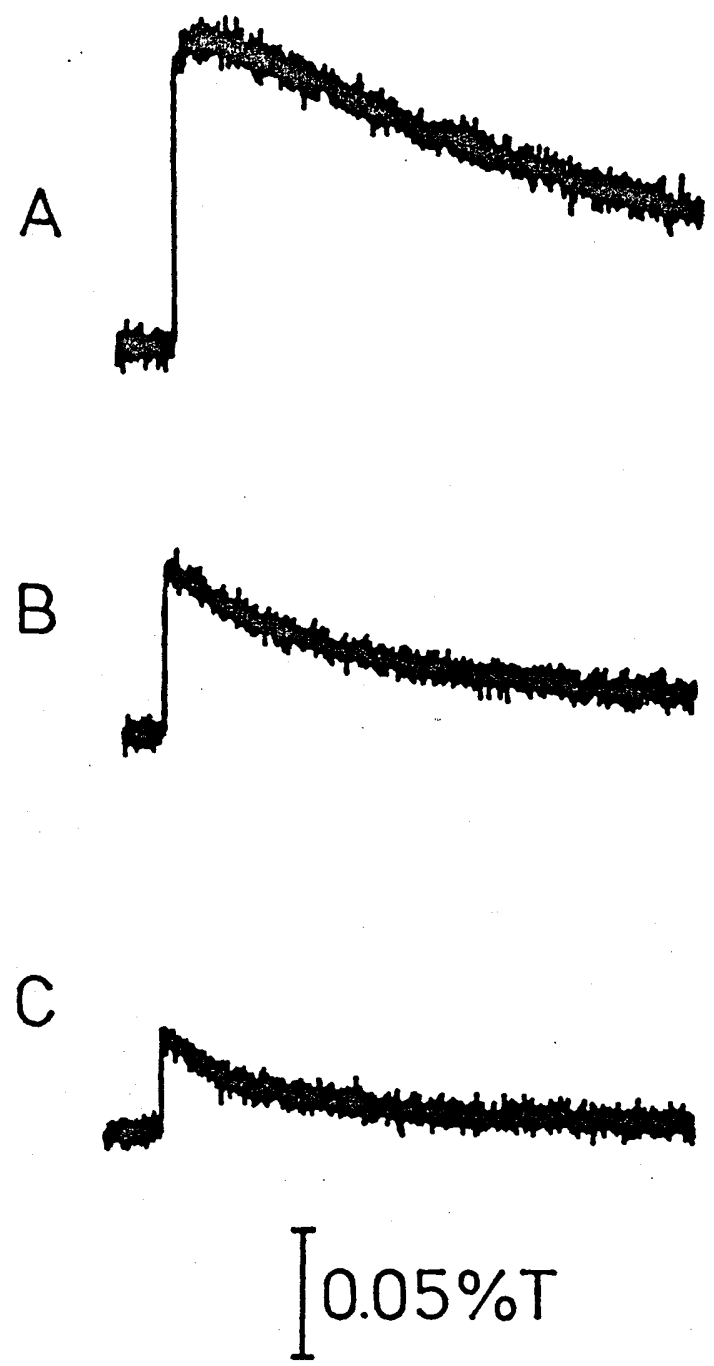

D

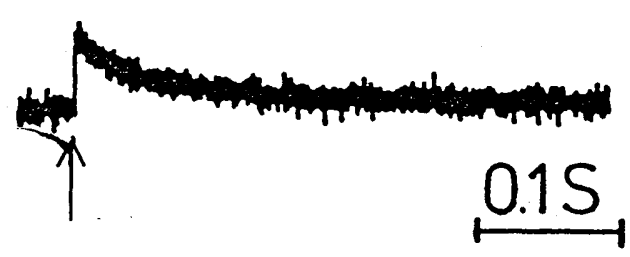

The effect of dark trypsin-treatment of thylakoids on the electrochromic bandshift. Thylakoids with a chlorophyll concentration of $25 \mathrm{Mgml}^{-1}$ were incubated with trypsin ( $5 \mu \mathrm{gml}^{-1}$ ) in $3.5 \mathrm{ml}$ of medium containing Tricine $(20 \mathrm{mM}), \mathrm{KCl}(10 \mathrm{mM})$ and $\mathrm{MgCl}_{2}$ ( $2 \mathrm{mM}$ ) $\mathrm{pH} 7.4$. The reaction was stopped by adding trypsin inhibitor $\left(0.5 \mathrm{mgml}^{-1}\right)$ and $\mathrm{K}_{3} \mathrm{Fe}(\mathrm{CN})_{6}(0.75 \mathrm{mM})$ was added as electron acceptor. Trypsin treatment: A) Zero;

B) $2 \mathrm{~min}$; C) $6 \mathrm{~min}$; D) $10 \mathrm{~min}$. 


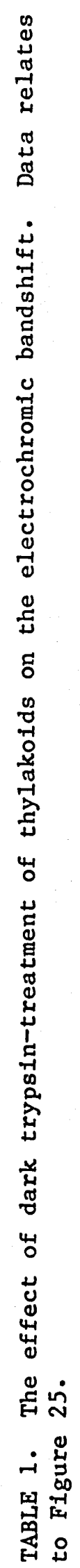

\begin{tabular}{|c|c|c|c|c|}
\hline 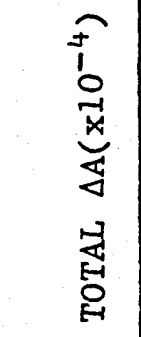 & $\stackrel{?}{0}$ & $\begin{array}{l}\dot{p} \\
\dot{m}\end{array}$ & $\stackrel{\sim}{\sim}$ & $\stackrel{9}{-1}$ \\
\hline 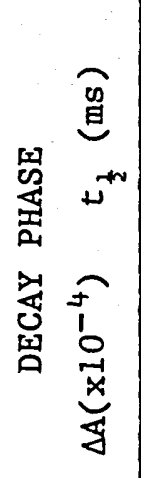 & & $\begin{array}{l}\tilde{0} \\
\infty \\
+1 \\
\ddot{0} \\
\infty \\
\tilde{m} \\
\sim \\
\dot{0} \\
+1 \\
\tilde{-}\end{array}$ & 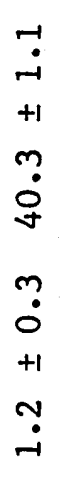 & $\begin{array}{l}0 \\
\dot{0} \\
+1 \\
m \\
\dot{0} \\
-1 \\
\dot{0} \\
+1 \\
-1 \\
-1\end{array}$ \\
\hline 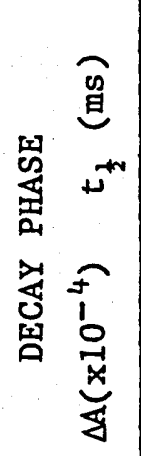 & $\begin{array}{l}n \\
+1 \\
0 \\
\dot{0} \\
\text { i } \\
\because \\
\dot{0} \\
+1 \\
? \\
\dot{j}\end{array}$ & 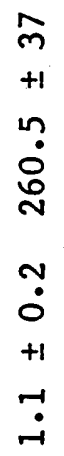 & & \\
\hline 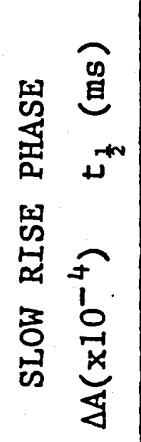 & $\begin{array}{l}\tilde{-} \\
+1 \\
\ddot{\infty} \\
\ddot{0} \\
+1 \\
\ddot{+}\end{array}$ & & & \\
\hline 量 & 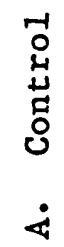 & $\begin{array}{l}\underset{H}{H} \\
N \\
N \\
\dot{N}\end{array}$ & $\begin{array}{l}\frac{I}{\dot{H}} \\
0 \\
\dot{0}\end{array}$ & $\begin{array}{l}\vec{H} \\
\dot{\theta} \\
\dot{\circ} \\
\dot{\theta}\end{array}$ \\
\hline
\end{tabular}



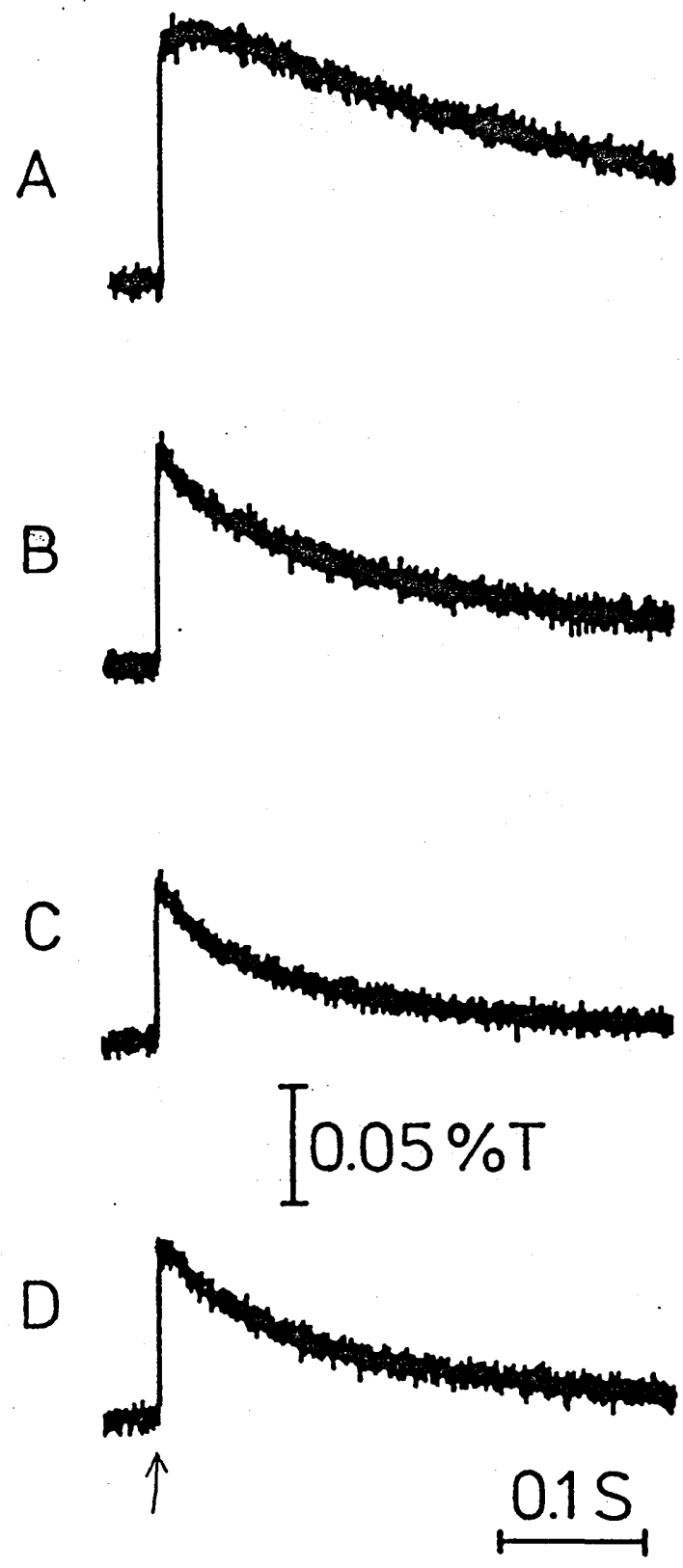

F IGURE 26 .

The effect of dark chymotrypsin-treatment of thylakoids on the electrochromic bandshift. Thylakoids with a chlorophyll concentration of $25 \mathrm{Mgml}^{-1}$ were incubated with chymotrypsin $\left(15 \mu^{-1}\right)$ in $3.5 \mathrm{ml}$ of medium containing Tricine $(20 \mathrm{mM}), \mathrm{KCl}(10 \mathrm{mM})$ and $\mathrm{MgCl}_{2}(2 \mathrm{mM})$ $\mathrm{pH}$ 7.4. The reaction was stopped by adding trypsin inhibitor $\left(0.5 \mathrm{mgmi}^{-1}\right)$ and $\mathrm{K}_{3} \mathrm{Fe}(\mathrm{CN})_{6}$ $(0.75 \mathrm{mM})$ was added as electron acceptor. Chymotrypsin treatment; A) Zero; B) $2 \mathrm{~min}$; C) $6 \mathrm{~min}$; D) $10 \mathrm{~min}$. 


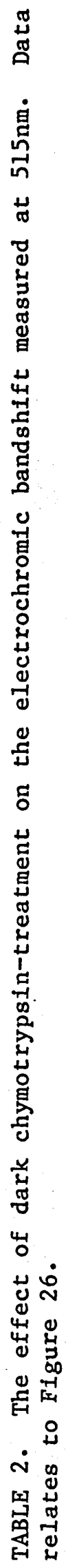

\begin{tabular}{|c|c|c|c|c|c|}
\hline & 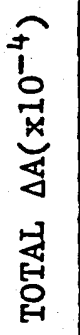 & in & $\dot{q}$ & $\begin{array}{l}\stackrel{\leftrightarrow}{m} \\
\dot{m}\end{array}$ & $\stackrel{r}{m}$ \\
\hline 贸 & 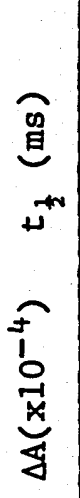 & & $\begin{array}{l}n \\
+1 \\
\infty \\
\tilde{-1} \\
-1 \\
\dot{0} \\
+1 \\
\tilde{n} \\
\dot{0}\end{array}$ & $\begin{array}{l}m \\
+1 \\
-1 \\
\dot{a} \\
-1 \\
\ddot{0} \\
+1 \\
0 \\
\ddot{0} \\
\dot{-1}\end{array}$ & \\
\hline 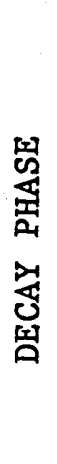 & 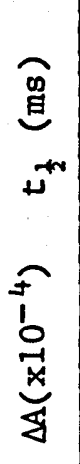 & $\begin{array}{l}n \\
+1 \\
0 \\
\dot{0} \\
\infty \\
\sim\end{array}$ & $\begin{array}{l}0 \\
+1 \\
+ \\
\dot{n} \\
\ddot{r} \\
\ddot{0} \\
\dot{+} \\
\ddot{+1} \\
\ddot{m}\end{array}$ & $\begin{array}{l}n \\
+1 \\
n \\
\tilde{a} \\
\tilde{-1} \\
-1 \\
\dot{0} \\
+1 \\
\infty \\
\dot{-1}\end{array}$ & $\begin{array}{l}\text { - } \\
+1 \\
+ \\
\dot{0} \\
+ \\
0 \\
\dot{0} \\
+1 \\
\bullet \\
\dot{v}\end{array}$ \\
\hline 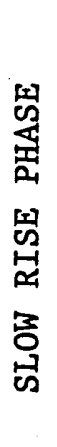 & 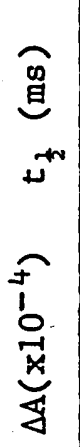 & $\begin{array}{c}N \\
+1 \\
r \\
-1 \\
\tilde{H} \\
\dot{0} \\
+1 \\
\tilde{\sigma} \\
\dot{0}\end{array}$ & & & \\
\hline 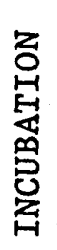 & 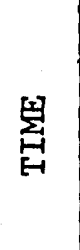 & 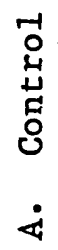 & $\begin{array}{l}\text { 品 } \\
\text { 百 } \\
\sim \\
\dot{N}\end{array}$ & $\begin{array}{l}\text { E } \\
\stackrel{-1}{E} \\
0 \\
ن\end{array}$ & 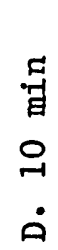 \\
\hline
\end{tabular}


of $176.4 \pm 6 \mathrm{~ms}$ and a fast phase with a half-time of decay of $17.8 \pm 3 \mathrm{~ms}$ (Fig $26(\mathrm{~B})$ and Table $2(\mathrm{~B})$ ) these constituting 76 and $24 \%$ of the signal respectively. on completion of $10 \mathrm{~min}$ incubation with chymotrypsin only one phase of decay was seen, this having a halftime decay of $66.4 \pm 11 \mathrm{~ms}$ (Fig 26(D). and Table 2 (D)). chymotrypsin had a much less marked effect on the extent than trypsin with $69 \%$ of the signal remaining after $10 \mathrm{~min}$ chymotrypsin treatment compared to $30 \%$ in similar trypsin treated samples.

2. Incubation with trypsin or chymotrypsin in the light. Trypsin incubation in white 1 ight $\left(120 \mathrm{wm}^{-2}\right)$ caused two changes in the electrochromic bandshift (Fig. 27): 1) the half-time decay was decreased and 2) the extent was reduced. The effects observed here were similar to those observed after trypsin incubation in the dark (Fig. 25).

The curve fitting data ( Table 3 ) shows that the lighttreated control (A) had no slow rise phase and decayed with one phase, which had a half-time of $141 \pm 11 \mathrm{~ms}$. After 10min incubation with trypsin (Fig. 27D and Table $3(D)$ ) the half-time decay has been decreased to $17.0 \pm 5.0 \mathrm{~ms}$. The extent of the bandshift was reduced considerably with on 1 y $31 \%$ of the control remaining after ten minutes incubation (Fig 27 ).

The results for chymotrypsin treatment in the light are given in Figure 28 and the curve fitting analysis is shown in Table 4. The half-time decay rate for the 1 ight-treated control was $141 \pm 11 \mathrm{~ms}$ (Fig $28 \mathrm{~A}^{\circ}$ and rable 4A). On completion of $10 \mathrm{~min}$ incubation with chymotrypsin the half-time decay was reduced to $96 \pm 4 \mathrm{~ms}$. 

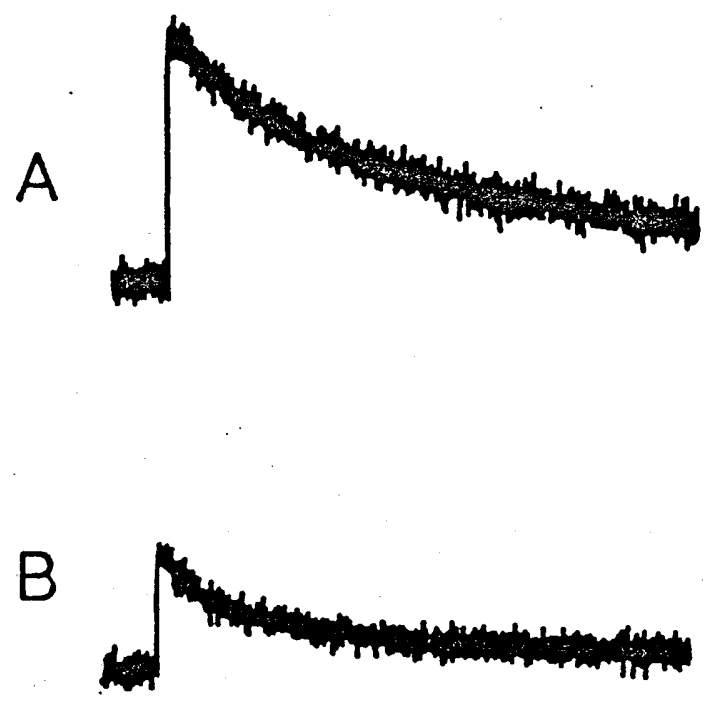

C
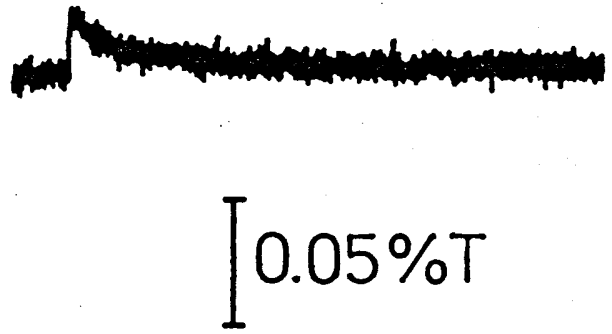

0

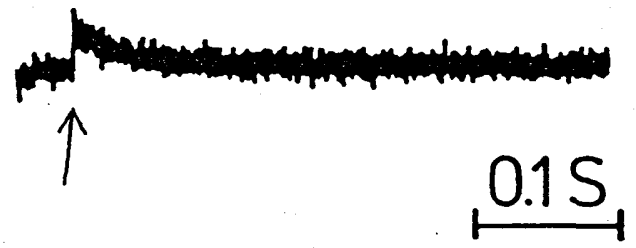

The effect of light $\left(120 \mathrm{Wm}^{-2}\right)$ trypsintreatment of thylakoids on the electrochromic bandshift. Thylakoids with a chlorophyll concentration of $25 \mu^{-1}$ were incubated with trypsin $(5 \mu \mathrm{gml}-1)$ in $3.5 \mathrm{ml}$ of medium containing Tricine $(20 \mathrm{mM}), \mathrm{KCl}(10 \mathrm{mM})$ and $\mathrm{MgCl}_{2}$ ( $\left.2 \mathrm{mM}\right) \mathrm{pH} 7.4$. The reaction was stopped by adding trypsin inhibitor $\left(0.5 \mathrm{mgmi}^{-1}\right)$ and $\mathrm{K}_{3} \mathrm{Fe}(\mathrm{CN})_{6}(0.75 \mathrm{mM})$ was added as electron acceptor. Trypsin treatment:
A) Zero:
B) $2 \mathrm{~min}$;
C) $6 \mathrm{~min}$;
D) $10 \mathrm{~min}$. 

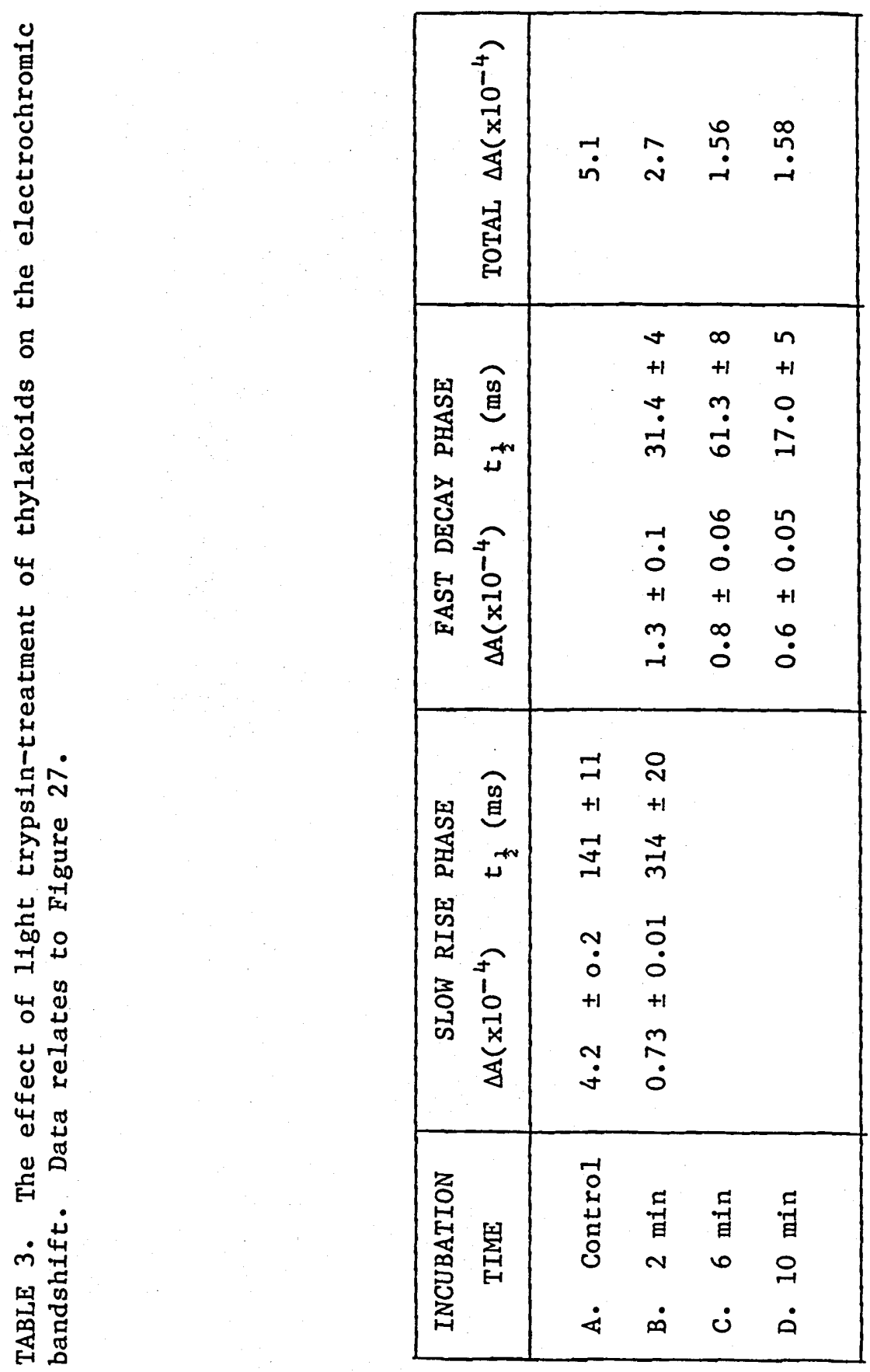

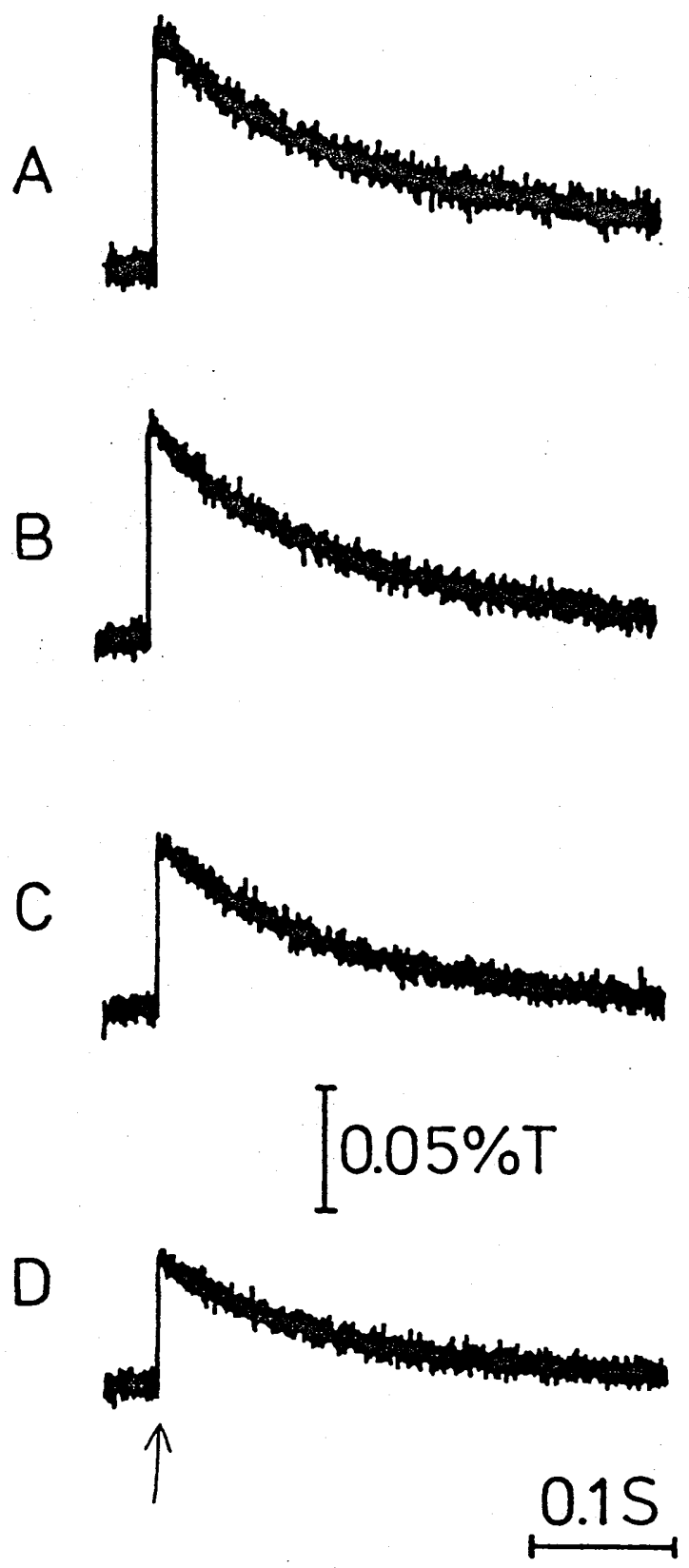

FIGURE 28 .

The effect of light $\left(120 \mathrm{Wm}^{-2}\right)$ chymotrypsintreatment of thylakoids on the electrochromic bandshift. Thylakoids with a chlorophyll concentration of $25 \mathrm{Mgmi}^{-1}$ were incubated with chymotrypsin $\left(15 \mathrm{Mgml}^{-1}\right)$ in $3.5 \mathrm{ml}$ of medium containing Tricine $(20 \mathrm{mM})$, KCl (10.mM) and $\mathrm{MgCl}_{2}(2 \mathrm{mM}) \mathrm{pH} \mathrm{7.4.} \mathrm{The}$ reaction was stopped by adding trypsin inhibitor: $\left(0.5 \mathrm{mgml}^{-1}\right)$ and $\mathrm{K} 3 \mathrm{Fe}(\mathrm{CN})_{6}$ $(0.75 \mathrm{mM})$ was added as electron acceptor. Chumotrypsin treatment; A) 7ero; B) $2 \mathrm{~min}$; C) $6 \mathrm{~min}$; D) $10 \mathrm{~min}$. 


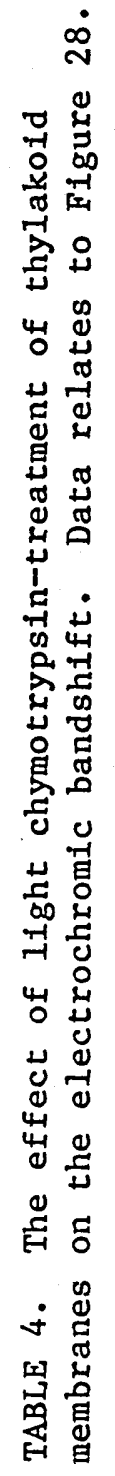

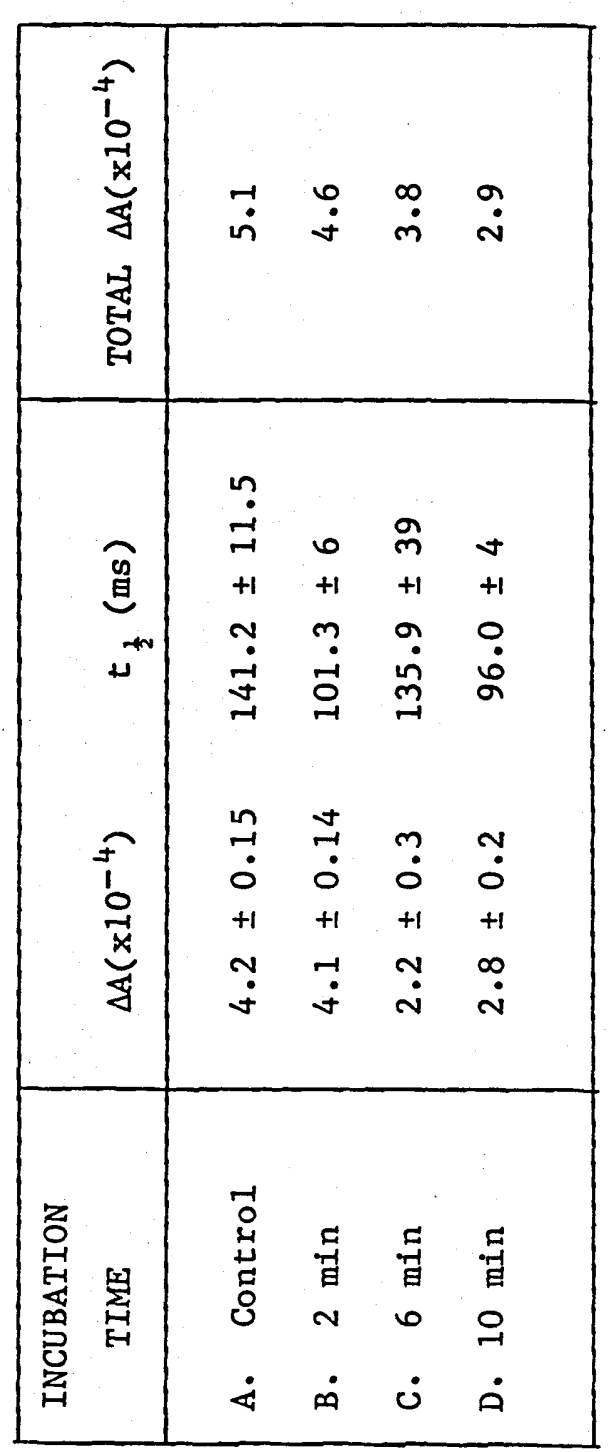


Here, as in the dark treated samples, chymotrypsin had a less marked effect on the extent than trypsin with 55 and $31 \%$ of the extent of the control remaining respectively.

Comparing the results of trypsin and chymotrypsin on light-and dark treated membranes shows that the effects of these enzymes have similar trends. However, it can be seen that the effect of chymotrypsin on the halftime of decay and on the extent of the bandshift, in all cases, is much less marked than that of trypsin.

Trypsin appeared to have a greater effect on 1 ighttreated compared to similar dark-treated membranes but care has to be taken in the interpretation of these results. It was shown in Figure 24 that 1 ight treatment alone affects the electrochromic bandshift signal, therefore it was not possible to make a direct comparison between the light-and dark-treated samples. Never-the-less it can be seen from these results that trypsin (Fig. 27) caused a further decrease in the half-time of decay on top of that caused by light treatment alone. Although chymotrypsin has a similar effect it was significantly less than that observed with trypsin. Moreover, in contrast to trypsin, chymotrypsin did not accelerate the rate of decay in the light-treated samples any further than was observed for similar dark-treated membranes.

It was concluded that trypsin, and to a lesser extent chymotrypsin, were altering the functions of the membrane in such a way that the ionic permeability of the membrane was increased. This was observed as a decrease in the decay half-time of the electrochromic bandshift and is most probably caused by enzymic digestion of one or more of the extrinsic membrane polypeptides. There is evidence to suggest that the ATPase complex may 
be a susceptible site on the membranes as a number of workers have shown that alterations in the function of this complex can alter the half-time of decay of the bandshift (Girault and Galmiche, 1978; Itoh and Morita, 1982 and Peters et al, 1983 ).

The reduction in the extent of the bandshift, induced by treatment of thylakoids with trypsin or chymotrypsin may be due to a number of factors and this is discussed in chapter 5 .

4.2.3 The effects of adenine nucleotides on the
electrochromic bandshift in thylakoids
pretreated with dithiothreitol, trypsin
and chymotrypsin.

Addition of ATP and ADP, to dark-adapated untreated thylakoid membranes, had no effect on the half-time decay of the electrochromic bandshift signal obtained from these samples (see Fig 29 A-C). However, if the thylakoids were first incubated in strong white light $\left(\sim 120 \mathrm{Wm}^{-2}\right)$ for $3 \mathrm{~min}$, addition of ATP in the subsequent dark period induced a slight decrease in the half-time decay (Fig. 29E), whilst addition of ADP still had no effect (Fig. 29F). The effect of dithiothreitol (DTT) on the electrochromic bandshift in thylakoids, treated in the dark or in strong light, is shown in Figure 30. It can be seen that neither ATP nor ADP had any effect on samples treated with dithiothreitol in the dark (Fig. $30 \mathrm{~A}-\mathrm{C}$ ). But Figure $30 \mathrm{E}$ shows that, in membranes incubated in the light in the presence of dithiothreitol, ATP caused a marked decrease in the half-time decay of the bandshift whilst $A D P$ had no effect $(30 F)$. It is known that DTT can stimulate ATP hydrolysis (Petrack et al, 1965) 

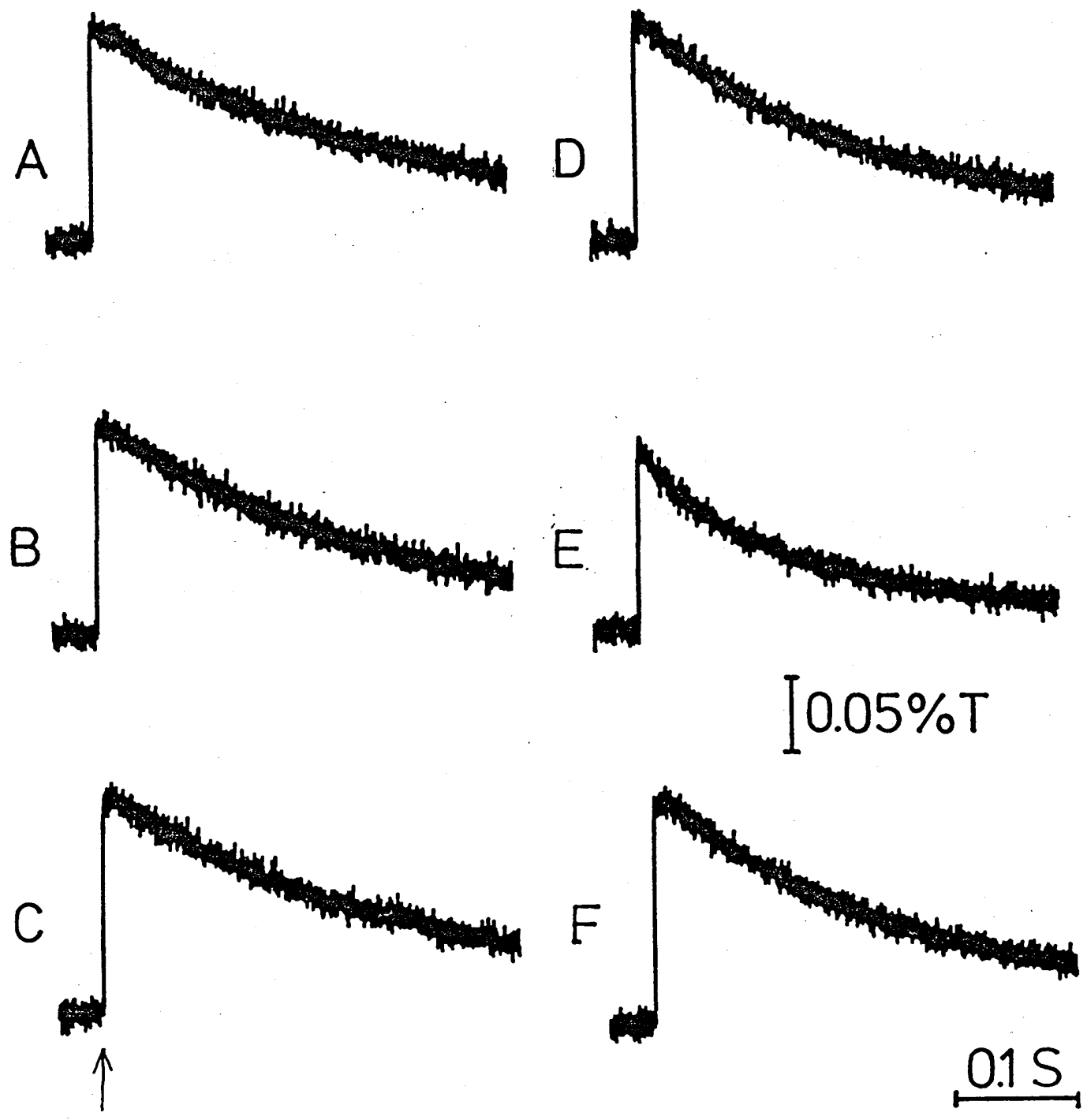

FIGURE 29 .

The effect of ATP and ADP on the electrochromic bandshift of isolated thylakoids. Thylakoids with a chlorophyll concentration of $25 \mathrm{Mgml}^{-1}$ were incubated for $3 \mathrm{~min}$ in the dark or $1 \mathrm{ight}$ $\left(\sim 120 \mathrm{Wm}^{-2}\right)$, in a medium containing Tricine $(20 \mathrm{mM}), \mathrm{KCl}(10 \mathrm{mM}), \mathrm{MgCl}_{2}(2 \mathrm{mM})$ and $\mathrm{K}_{2} \mathrm{HPO}_{4}(2.0 \mathrm{mM}), \mathrm{pH} 7.4$. On completion of incubation ATP $(0.5 \mathrm{mM})$ or ADP $(0.5 \mathrm{mM})$ was added, $\mathrm{K}_{3} \mathrm{Fe}(\mathrm{CN})_{6}(0.75 \mathrm{mM})$ was the electron acceptor. DARK TREATED: A) Control, no additions; B ) + ATP; C ) + ADP. LIGHT TREATED: D) Control no additions; E) + ATP;

F) + ADP. 

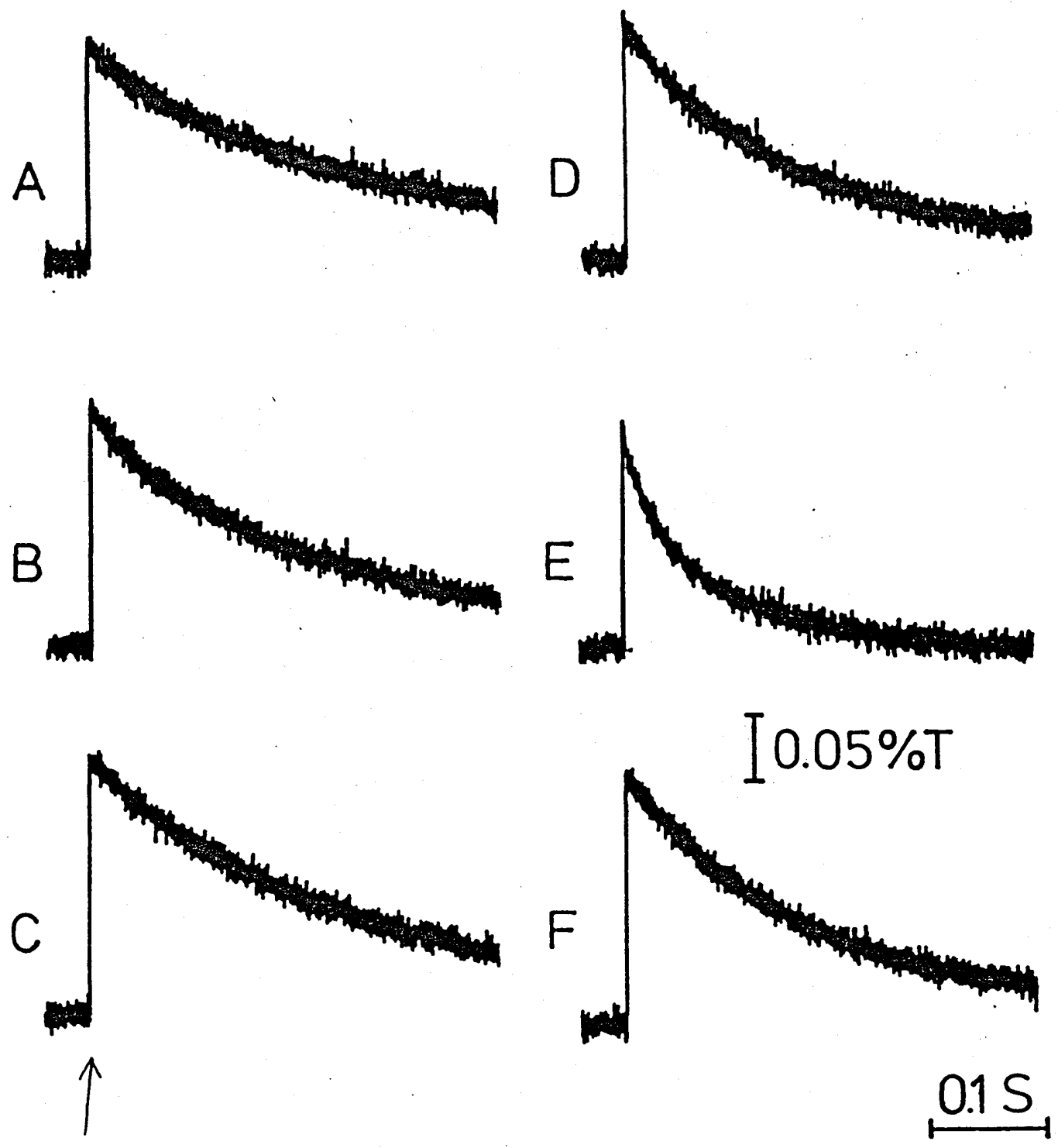

\section{F IGURE 30.}

The effect of ATP and ADP on the eiectrochromic bandshift of thylakoids pretreated with dithiothreitol. Thylakoids with a chlorophyll concentration of $25 \mu \mathrm{gml}-1$ were incubated with dithiothreitol $(5.0 \mathrm{mM})$, for $3 \mathrm{~min}$ in the dark or light $\left(\sim 120 \mathrm{wm}^{-2}\right)$, in a medium containing Tricine $(2 \mathrm{C} \mathrm{mM}), \mathrm{KCl}(10 \mathrm{mM})$, $\mathrm{MgCl}_{2}(2 \mathrm{mM})$ and $\mathrm{K}_{2} \mathrm{HPO}_{4}(2.0 \mathrm{mM}), \mathrm{pH} 7.4$. on completion of incubation ATP $(0.5 \mathrm{mM})$ or ADP $(0.5 \mathrm{mM})$ was added, $\mathrm{K}_{3} \mathrm{Fe}(\mathrm{CN})_{6}(0.75 \mathrm{mM})$ was the electron acceptor. DARK TREATED:

A) Control, no additions; B) + ATP; C) + ADP. LIGHT TREATED: D) Control, no additions;

E) + ATP; F) + ADP. 
therefore the effect of ATP (Fig 3OE) may be due to. the hydrolytic activity of the activated ATP hydrolase.

The effects of ATP and ADP on membranes which had been pretreated with trypsin or chymotrypsin are shown in Figures 31 and 32 respectively. It can be seen (c.f. Figs 29, and 30 ) that after dark pretreatment with trypsin or chymotrypsin neither ATP nor ADP had an affect on the electrochromic bandshift (Figs. 31 and 32). However, addition of ATP to thylakoids treated with trypsin or chymotrypsin in the light resulted in a marked decrease in the half-time of decay (Fig 31E and 32E); ADP had no effect (Fig. 31F and 32F). These results are similar to those obtained using dithiothreitol.

4.2.4 The effect of tentoxin on the ability of ATP to decrease the half-time of decay of the electrochromic bandshift, in thylakoids pretreated with dithiothreitol, trypsin and chymotrypsin.

Tentoxin is an inhibitor of ATP hydrolysis, provided that it is used at low concentrations $(\leqslant 2 \mu \mathrm{M})$ (steele et al, 1978). The effect of tentoxin $(2 \mu \mathrm{M})$ on the electrochromic bandshift in untreated thylakoid membranes is shown in Figure $33(\mathrm{~A}-\mathrm{C})$ : it can be seen that addition of ATP to light-treated control membranes caused a slight decrease in the half-time of decay of the electrochromic bandshift and that tentoxin inhibited this effect. Tentoxin was only able to act as an inhibitor if it was present during the 1 ight incubation period. 

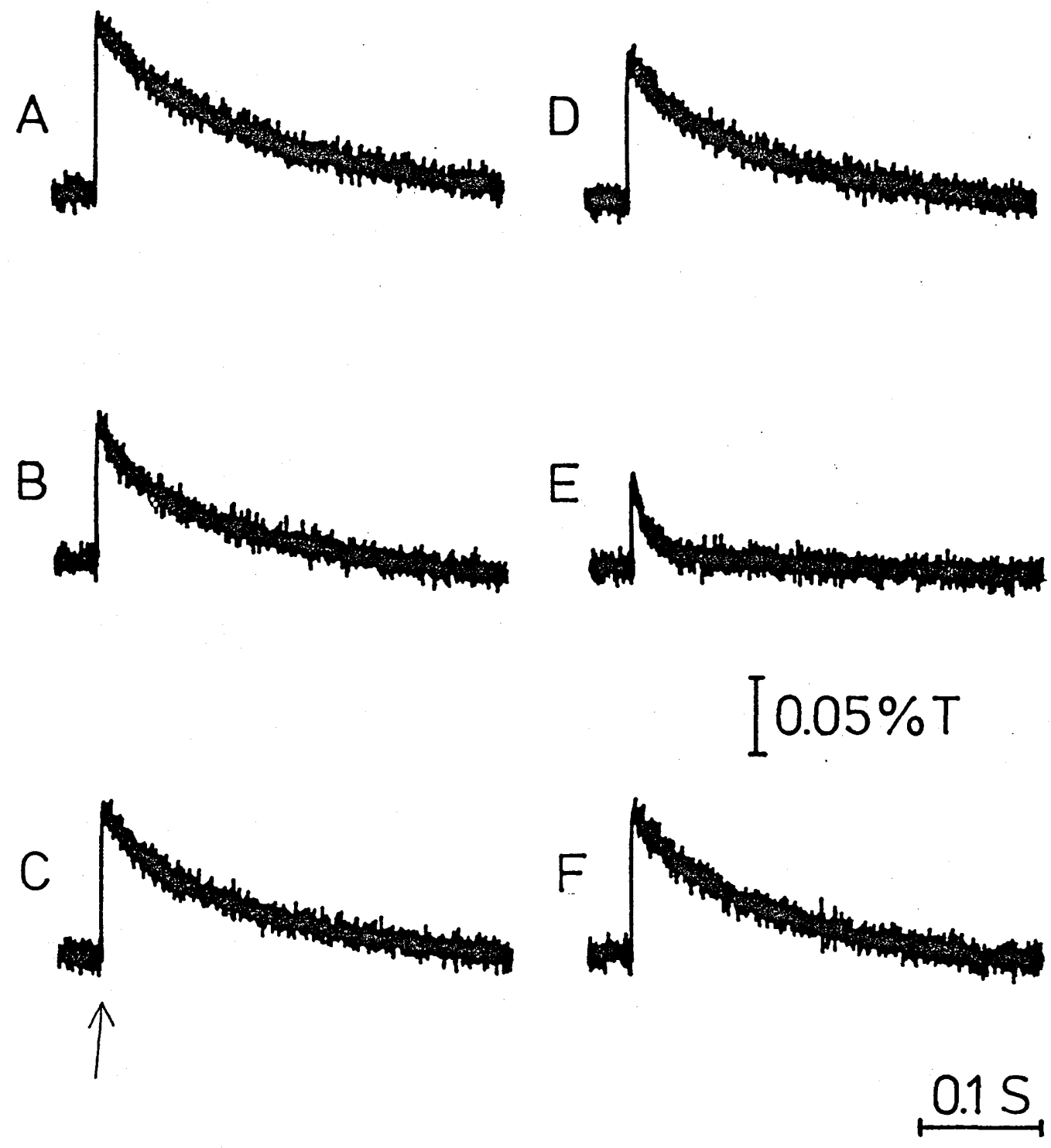

FIGURE 31.

The effect of ATP and ADP on the electrochromic bandshift of trypsintreated thylakoids. Thylakoids with a chlorophyll concentration of $25 \mu \mathrm{g} \mathrm{ml^{-1 }}$ were incubated for $1 \mathrm{~min}$ with trypsin $\left(5 \mu \mathrm{g} \mathrm{ml} \mathrm{m}^{-1}\right)$ in the dark or light $\left(120 \mathrm{Wm}^{-2}\right)$, in $3.5 \mathrm{ml}$ of medium containing Tricine $(20 \mathrm{mM}), \mathrm{KCl}(10 \mathrm{mM}), \mathrm{Mg} \mathrm{Cl} 2(2 \mathrm{mM})$ and $\mathrm{K}_{2} \mathrm{HPO}_{4}(2 \mathrm{mM}) \mathrm{pH} 7.4$. The reaction was stopped by adding trypsin inhibitor $\left(0.5 \mathrm{mg} \mathrm{ml^{-1 }}\right)$ then ATP $(0.5 \mathrm{mM})$ or $\mathrm{ADP}(0.5 \mathrm{mM})$ and $\mathrm{K}_{3} \mathrm{Fe}(\mathrm{CN})_{6}(0.75 \mathrm{mM})$ were added. Dark treated: A) 1 min trypsin, no additions; B) + ATP; C) + ADP. Light treated: D) $1 \mathrm{~min}$ trypsin, no additions;

E) + ATP; F) + ADP. 

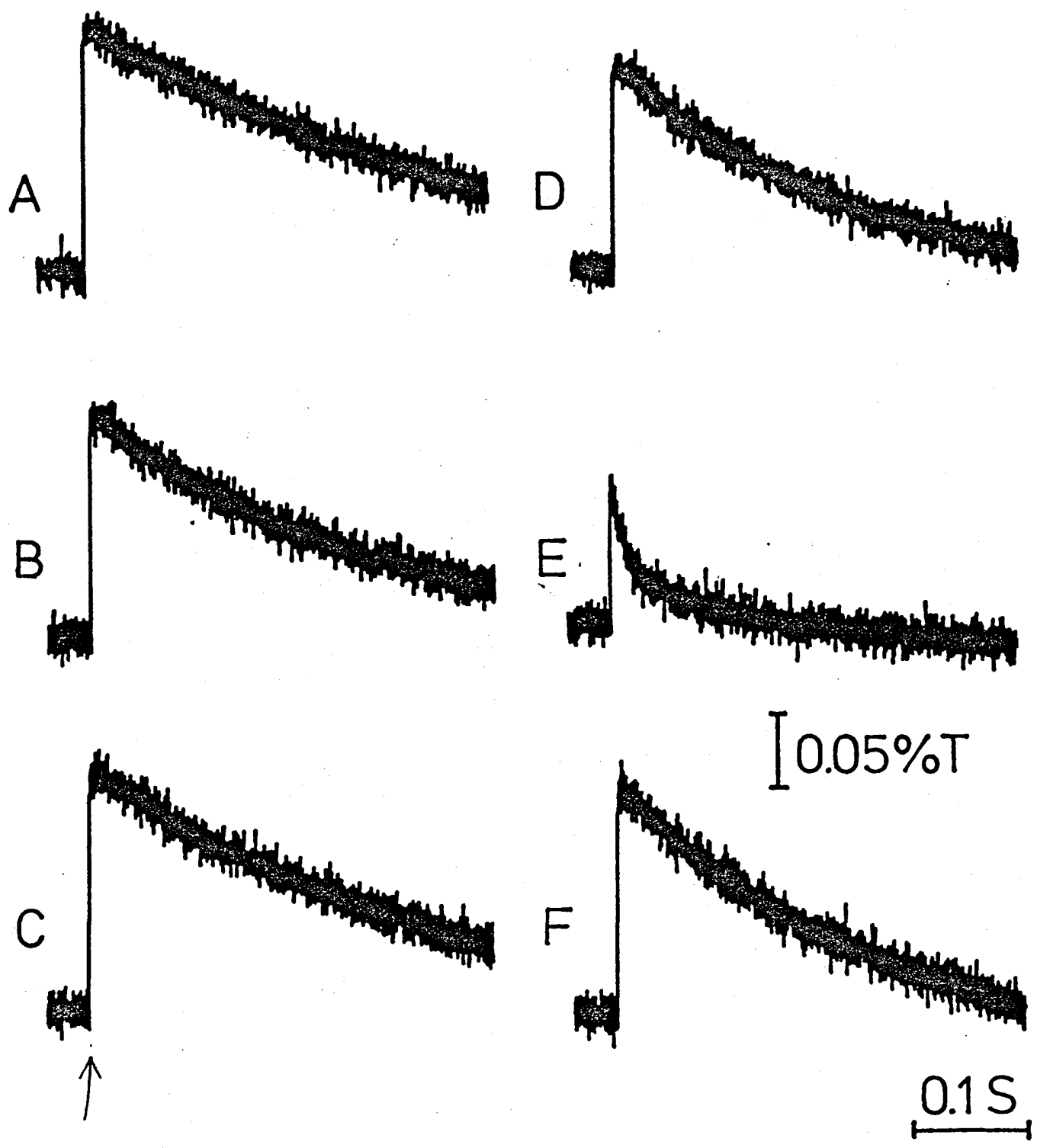

FIGURE 32 .

The effect of ATP and ADP on the electrochromic bandshift of chymotrypsin-treated thylakoid. Thylakolds with a chlorophyll concentration of $25 \mu \mathrm{g} \mathrm{ml^{-1 }}$ were incubated for $3 \mathrm{~min}$ with chymotrypsin

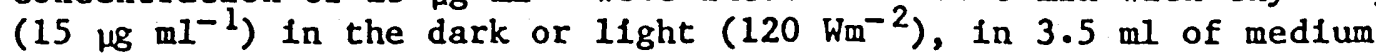
containing Tricine $(20 \mathrm{mM}), \mathrm{KCl}(10 \mathrm{mM}), \mathrm{Mg} \mathrm{Cl} 2(2 \mathrm{mM})$ and $\mathrm{K}_{2} \mathrm{HPO}_{4}$ $(2 \mathrm{mM}) \mathrm{pH} \mathrm{7.4}$. The reaction was stopped by adding trypsin inhibitor $\left(0.5 \mathrm{mg} \mathrm{ml}^{-1}\right)$ then ATP $(0.5 \mathrm{mM})$ or ADP $(0.5 \mathrm{mM})$ and $\mathrm{K}_{3} \mathrm{Fe}(\mathrm{CN})_{6}$ were added. Dark treated: A) 3 min chymotrypsin, no additions; B) + ATP; C) + ADP. Light treated: D) 3 min chymotrypsin; E) + ATP; F) + ADP. 

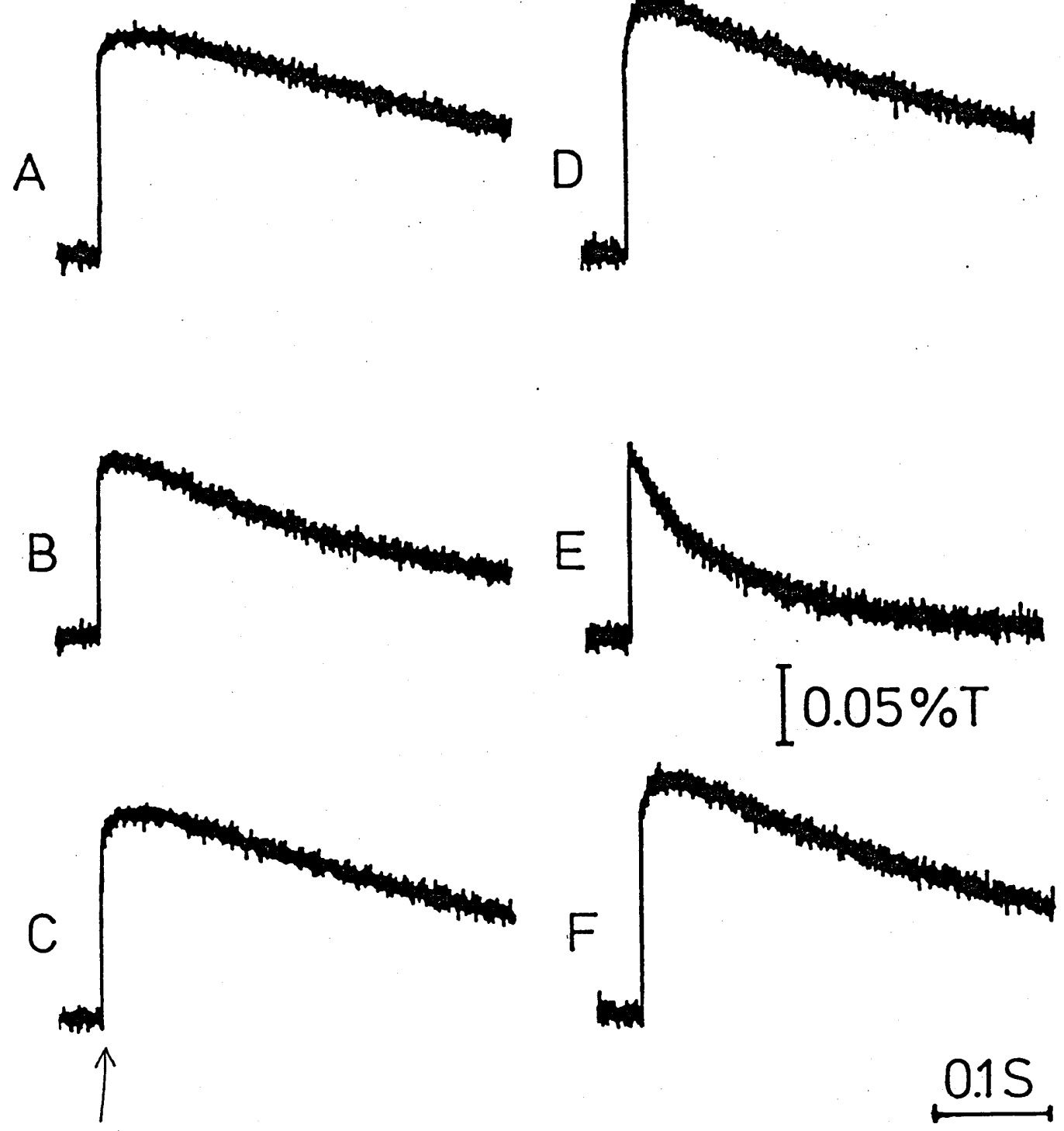

FIGURE 33 .

The effect of tentoxin on the electrochromic bandshift of thylakoids treated with

dithiothreitol in the light $\left(120 \mathrm{wm}^{-2}\right)$.

Thylakoids with a chlorophyll concentration

of $25 \mathrm{Mgml}^{-1}$ were incubated either in the

light alone or in the light with dithiothreitol

$(5.0 \mathrm{mM}$ ) and tentoxin $(2.0 \mu \mathrm{M}$ ) (where

indicated) for $3 \mathrm{~min}$ in a medium containing

$T$ ricine $(20 \mathrm{mM}), \mathrm{KCl}(10 \mathrm{mM}), \mathrm{MgCl}_{2}(2 \mathrm{mM})$

and $\mathrm{K}_{2} \mathrm{HPO}_{4}(2 \mathrm{mM}), \mathrm{pH} 7.4$. On completion of incubation ATP $(0.5 \mathrm{mM})$ was added then $\mathrm{K}_{3} \mathrm{Fe}(\mathrm{CN})_{6}$ $(0.75 \mathrm{mM})$. Controls: A) no additions; B) + ATP;

C) + ATP and tentoxin. Dithiothreitol treated:

D) no additions; E) + ATP; F) + ATP and tentoxin. 

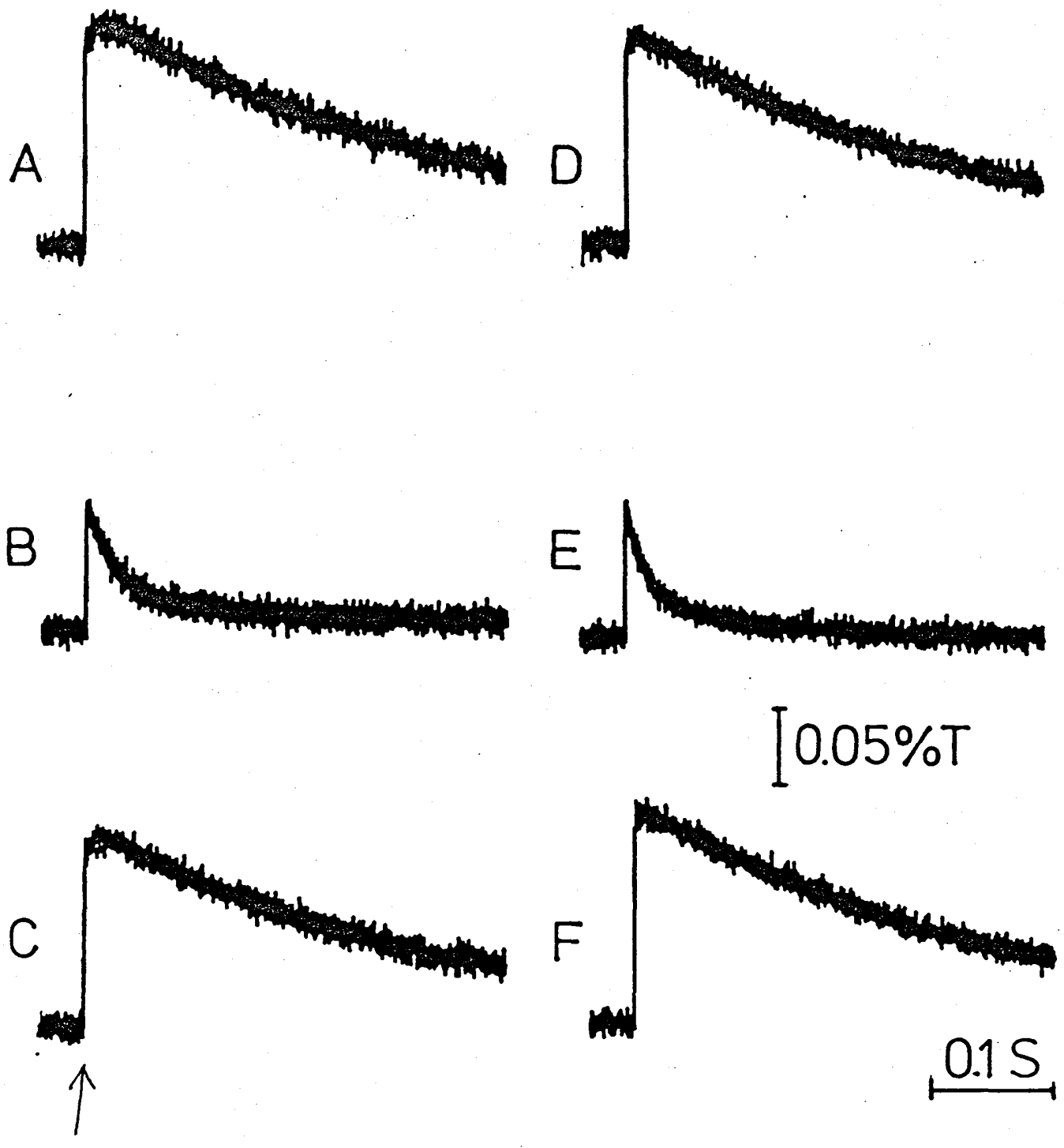

FIGIIRE 34 .

The effect of tentoxin on the electrochromic bandshift of thylakoids treated with trypsinor chymotrypsin in the light $\left(120 \mathrm{wm}^{-2}\right)$.

Thylakoids with a chlorophyll concentration of $25 \mathrm{Mgml}^{-1}$ were incubated with tentoxin $(2.0 \mu \mathrm{M})$ (where indicated) in the presence of trypsin $\left(5 \mathrm{Mgmi}^{-1}\right)$ for $1 \mathrm{~min}$ or with chymotrypsin

$\left(15 \mu \mathrm{gml}^{-1}\right)$ for $3 \mathrm{~min}$, in a medium containing Tricine $(20 \mathrm{mM}), \mathrm{KCl}(10 \mathrm{mM}), \mathrm{MgCl}_{2}(2 \mathrm{mM})$ and $\mathrm{K}_{2} \mathrm{HPO}_{4}$ ( $\left.2 \mathrm{mM}\right)$ pH 7.4. On completion of incubation trypsin inhibitor was added $\left(0.5 \mathrm{mgml}^{-1}\right)$ followed by ATP $(0.5 \mathrm{mM})$ then $\mathrm{K}_{3} \mathrm{Fe}(\mathrm{CN})_{6}(0.75 \mathrm{mM})$. Trypsin: A) no additions; B) + ATP; C) + ATP and : tentoxin. Chymotrypsin: D) no additions; E) + ATP $(F)+A T P$ and tentoxin 
As was shown previously (Fig. 30) incubation of thylakoids with dithiothreitol in the light, resulted in a marked decrease in the half-time of decay when ATP was added. However, the presence of tentoxin during the light pretreatment with dithiothreitol inhibited this effect of ATP (Fig.33 $(D-F))$.

The effect of tentoxin on trypsin and chymotrypsin treated thylakoids is shown in Figure 34. ATP was shown previously to accelerate the rate of decay of the electrochromic bandshift in thylakoids pretreated with either trypsin or chymotrypsin in the light (Figs 31 and 32 ). Tentoxin was seen to inhibit this effect of ATP in both trypsin-(Fig 34 (A-C)) and chymotrypsin- treated (Fig 34 (D-F)) samples.

\subsubsection{The effect of trypsin and chymotrypsin on ATP hydrolysis.}

Measurements of ATP hydrolysis were made on thylakoids which had been treated with trypsin or chymotrypsin: the conditions used were the same as those which caused the ATP-induced decrease in the half-time of decay of the electrochromic bandshift (4.2.3 Fig. 31 and 32 ). The results in Table 5 show that incubation of thylakoids with trypsin in strong light $\left(120 \mathrm{Wm}^{-2}\right)$ resulted in a stimulation of ATP hydrolysis by a factor of 3.5. A basal rate of hydrolysis was observed in the control samples even though the chloroplasts were given no pretreatments

(this result ties in with the slight decrease in the half-time decay of the electrochromic bandshift 
TABLE 5. The effect of trypsin on the rate of hydrolysis.

\begin{tabular}{|c|c|}
\hline INCUBATION CONDITIONS & $\begin{array}{c}\text { HYDROLYSIS RATE } \\
\left(\mathrm{H}^{+} \mathrm{ng} \mathrm{min}^{-1} \mathrm{mg} \mathrm{Ch1^{-1 }}\right)\end{array}$ \\
\hline $\begin{array}{l}\text { Control no Trypsin } \\
1 \text { min Light }+ \text { Trypsin } \\
1 \text { min Dark + Trypsin }\end{array}$ & $\begin{array}{l}200 \pm 24.3 \\
698 \pm 60.9 \\
130 \pm 29.3\end{array}$ \\
\hline
\end{tabular}

TABLE 6. The effect of chymotrypsin on the rate of hydrolysis.

\begin{tabular}{|l|c|}
\hline INCUBATION CONDITIONS & $\begin{array}{c}\text { HYDROLYSIS RATE } \\
\left(\mathrm{H}^{+} \mathrm{ng} \mathrm{min}-1 \mathrm{mg} \mathrm{ChI}{ }^{-1}\right)\end{array}$ \\
\hline Control no Chymotrypsin & $165 \pm 25.8$ \\
1 min Light + Chymotrypsin & $405 \pm 36.7$ \\
1 min Dark + Chymotrypsin & $177 \pm 39.4$ \\
\hline
\end{tabular}

Hydrolysis rates were measured by following $\mathrm{pH}$ changes induced by addition of ATP to thylakoid membranes. Thylakoids with a chlorophyll concentration of $25 \mu \mathrm{g} \mathrm{m} 1^{-1}$ were treated with trypsin $\left(5 \mu \mathrm{g} \mathrm{ml^{-1 } )}\right.$ or chymotrypsin $\left(15 \mu \mathrm{g} \mathrm{ml^{-1 }}\right)$ in the dark or in strong light $\left(120 \mathrm{Wm}^{-2}\right)$ in $3.5 \mathrm{ml}$ of medium containing $\mathrm{NaCl}(50 \mathrm{mM}), \mathrm{K}_{2} \mathrm{HPO}_{4}(2 \mathrm{mM})$ and $\mathrm{MgCl}_{2}(2 \mathrm{mM}), \mathrm{pH} 7.4$. On completion of incubation trypsin

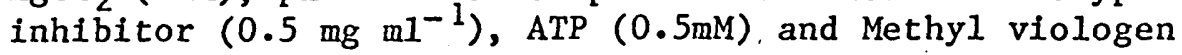
$(1.0 \mu \mathrm{m})$ were added.

To convert $H^{+}$to ATP multiply by a factor of .925 . 
induced by addition of ATP to light-treated:

control thylakoids (see 4.2.3 Fig. 29)). A slightly

slower rate of hydrolysis occurred in thylakoids

which had been pretreated with trypsin in the dark compared to that of the controls.

Table 6 shows the effect of chymotrypsin on ATP hydrolysis; it can be seen that these results show similar trends to the results observed with trypsin (Table 5). Comparing the rates of ATP hydrolysis in the controls with that of the 'light-treated chymotrypsin samples shows that chymotrypsin stimulated the rate of ATP hydrolysis by a factor of 2.5. The dark-treated. chymotrypsin samples gave similar rates to that of the controls.

This result indicated that both trypsin and chymotrypsin, under the conditions investigated here, had the ability to stimulate ATP hydrolysis. Lynn and straub (1969) showed that trypsin could stimulate ATP hydrolysis but these workers indicated that chymotrypsin had an inhibitory effect. In contrast, it was shown here that chymotrypsin, under certain conditions, can stimulate ATP hydrolysis.

4.2.6 The effects of trypsin and chymotrypsin on ATP synthesis.

Thylakoid membranes were treated with trypsin or chymotrypsin, either in the dark or in strong 1 ight $\left(120 \mathrm{Wm}^{-2}\right)$, and subsequently measurements were made of the rates of phosphorylation and oxygen evolution ( $\mathrm{H}_{2} \mathrm{O}$ to methyl viologen). The effects of trypsin on the rate of phosphorylation and oxygen evolution are shown in Table 7. From these data it can be seen 
that trypsin had a different effect on dark-and light-treated samples: in the dark-treated thylakoids $66 \%$ of the control rate of phosphorylation remained on completion of $5 \mathrm{~min}$ trypsin treatment whilst in similar light-treated samples complete inhibition occurred. Moreover $1 \mathrm{~min}$ incubation with trypsin in the light stimulated the rate of phosphorylation and also stimulated the rate of oxygen evolution; these effects were not seen in dark-treated samples. This trypsin-induced increase in phosphorylation may be due to activation of the ATPase enzyme induced by movement (or removal) of the $\varepsilon$ sub-unit of $\mathrm{CF}_{1}$ (see 1.5) which is believed to be an inhibitor of ATP synthesis ( Nelson:et a, 1972$)$. Table 7 shows the effects of trypsin on oxygen evoltuion using methyl viologen as an electron acceptor; these measurements were made simultaneously with the phosphorylation measurements. It is evident from these data (Table 7 ) that oxygen evolution was inhibited by trypsin in both dark- and light-treated samples with 92 and $82 \%$ of the control rate remaining respectively after $5 \mathrm{~min}$ incubation with trypsin. This decrease in the rate of electron transport (measured as oxygen evolution) is probably not sufficient to account for the marked decrease in phosphorylation.

Table 8 shows that chymotrypsin inhibited phosphorylation in both dark- and light-treated thylakoids: the effect in light-treated samples was more marked than in similar dark treated samples. After $5 \mathrm{~min}$ incubation with chymotrypsin $58 \%$ of the control rate of phosphorylation remained in dark- treated thylakoids whilst only $36 \%$ remained in 1 ight-treated membranes. These results show similar trends as were seen with trypsin ( Table 7) but chymotrypsin (Table 8) appeared 


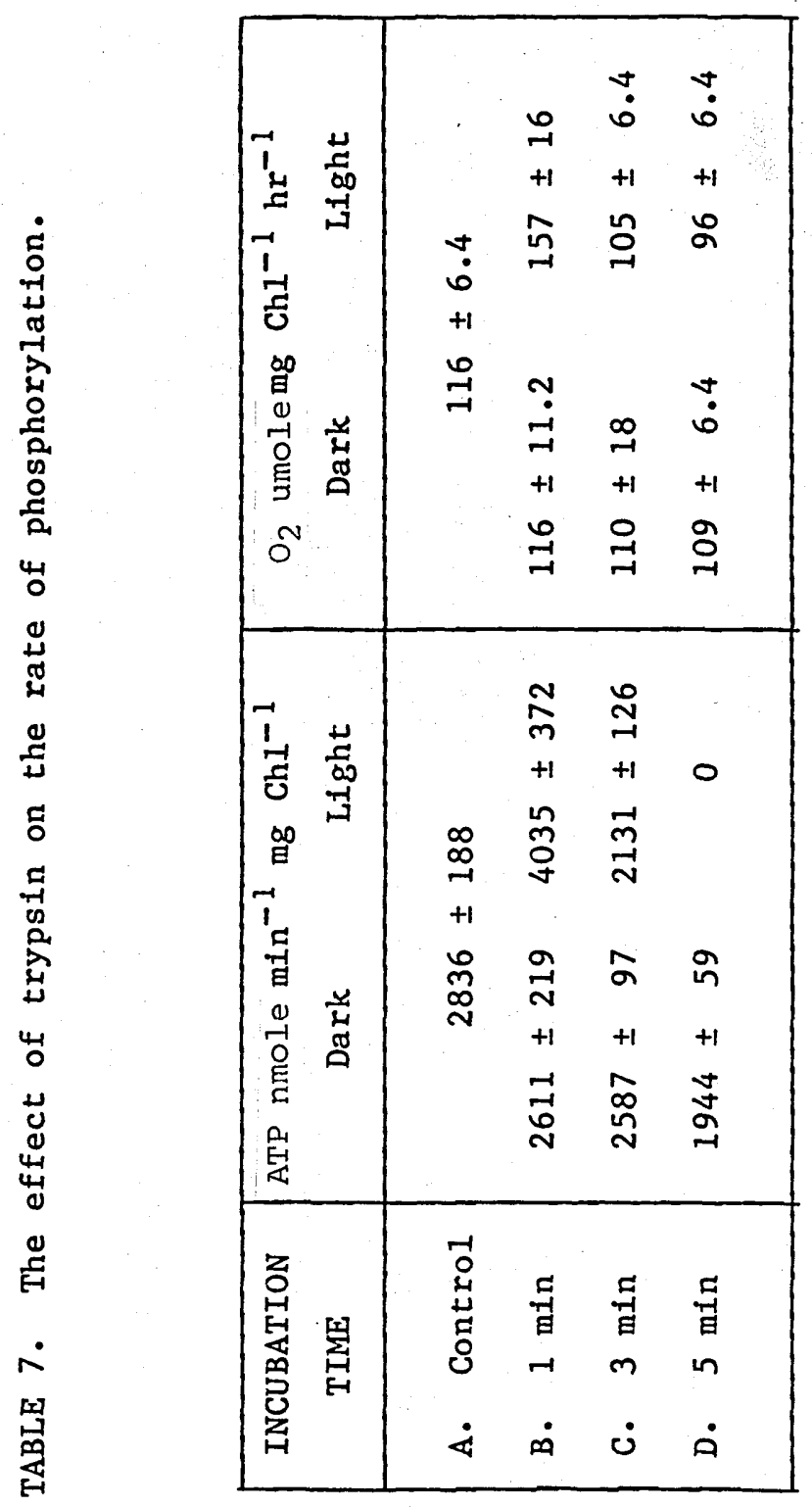

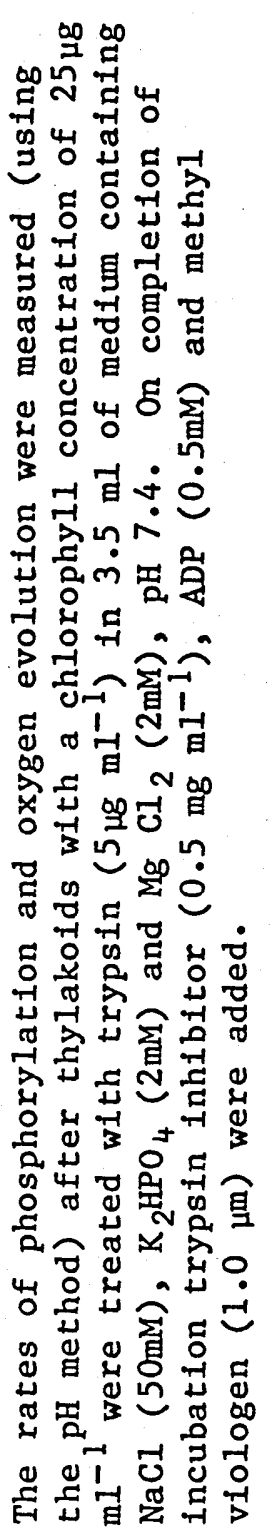



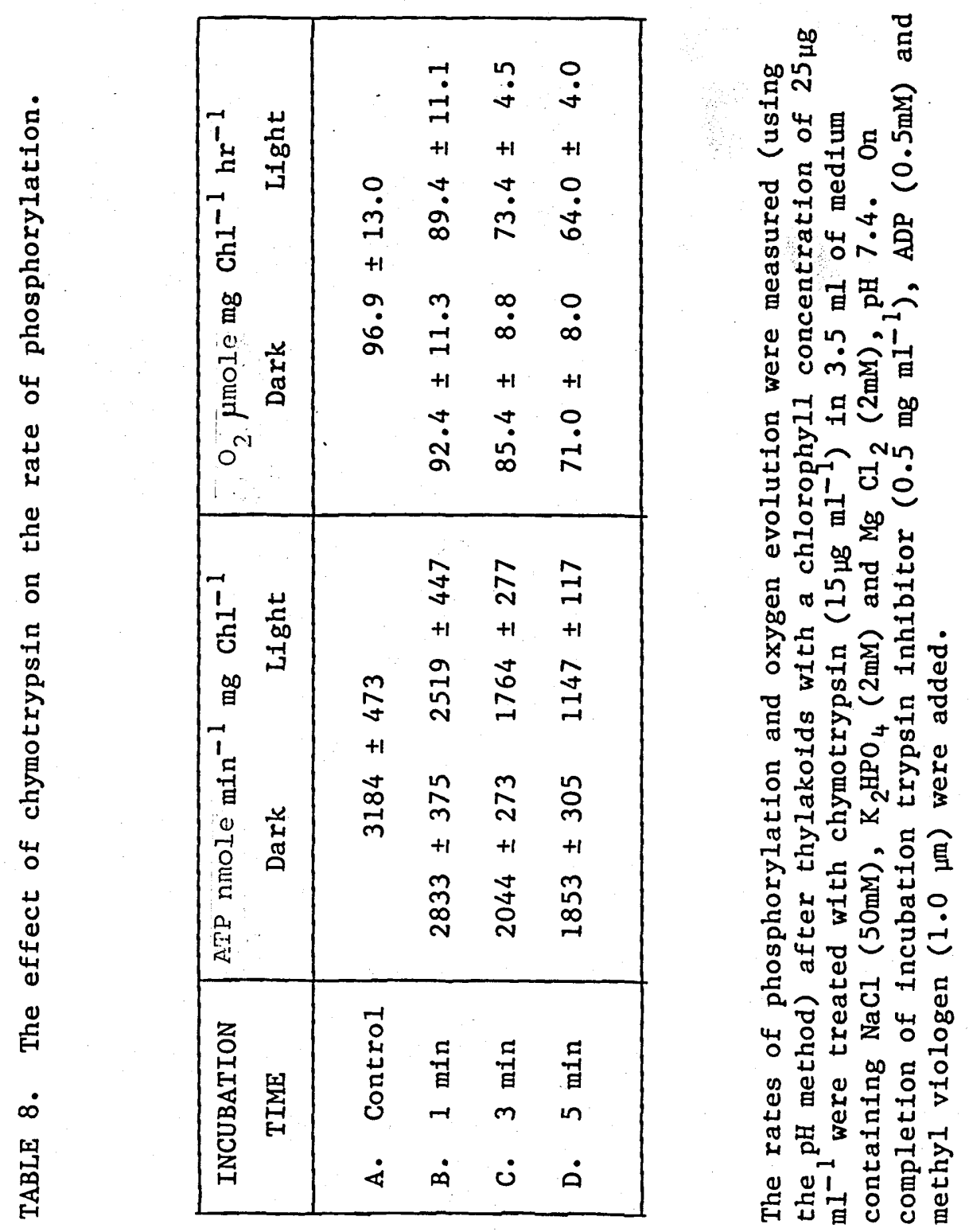
to have a more marked effect on the dark-treated membranes than trypsin, whilst trypsin had a more marked effect on the light- treated samples than chymotrypsin.

The oxygen evolution data for chymotrypsin (Table 8) showed that chymotrypsin inhibited the rate of oxygen evolution in both dark- and light-treated samples with 73 and $66 \%$ of the control rates remaining respectively. This result indicated that chymotrypsin may, in part, inhibit phosphorylation indirectly by inhibiting electron transport. In addition a direct inhibition of phosphorylation due to chymotrypsin incubation probably involves partial digestion of $\mathrm{CF}_{1}$.

4.2.7 The effect of ATP on 9-Aminoacridire fluorescence in thylakoids pretreated with trypsin and chymotrypsin.

In these experiments the 9-Aminoacridine fluorescence was excited by a weak measuring beam at $400 \mathrm{~nm}$, whilst photosynthesis was excited by an actinic beam of saturating red light. on excitation of the thylakoids with the actinic beam a decrease in 9-Aminoacridine fluorescence was seen (Fig 35 ). This decrease is due to membrane energisation and proton pumping induced by the 1ight; the decrease in 9-Aminoacridine fluorescence was inhibited by gramicidin which uncouples the thylakoid membrane by dissipating the proton gradient. Addition of ATP to thylakoid membranes in the dark resulted in a rapid decrease in the level of 9-Aminoacridine fluorescence (Fig 35); ATP has a direct quenching effect on the 9-Aminoacridine fluorescence and this is not affected by uncouplers 


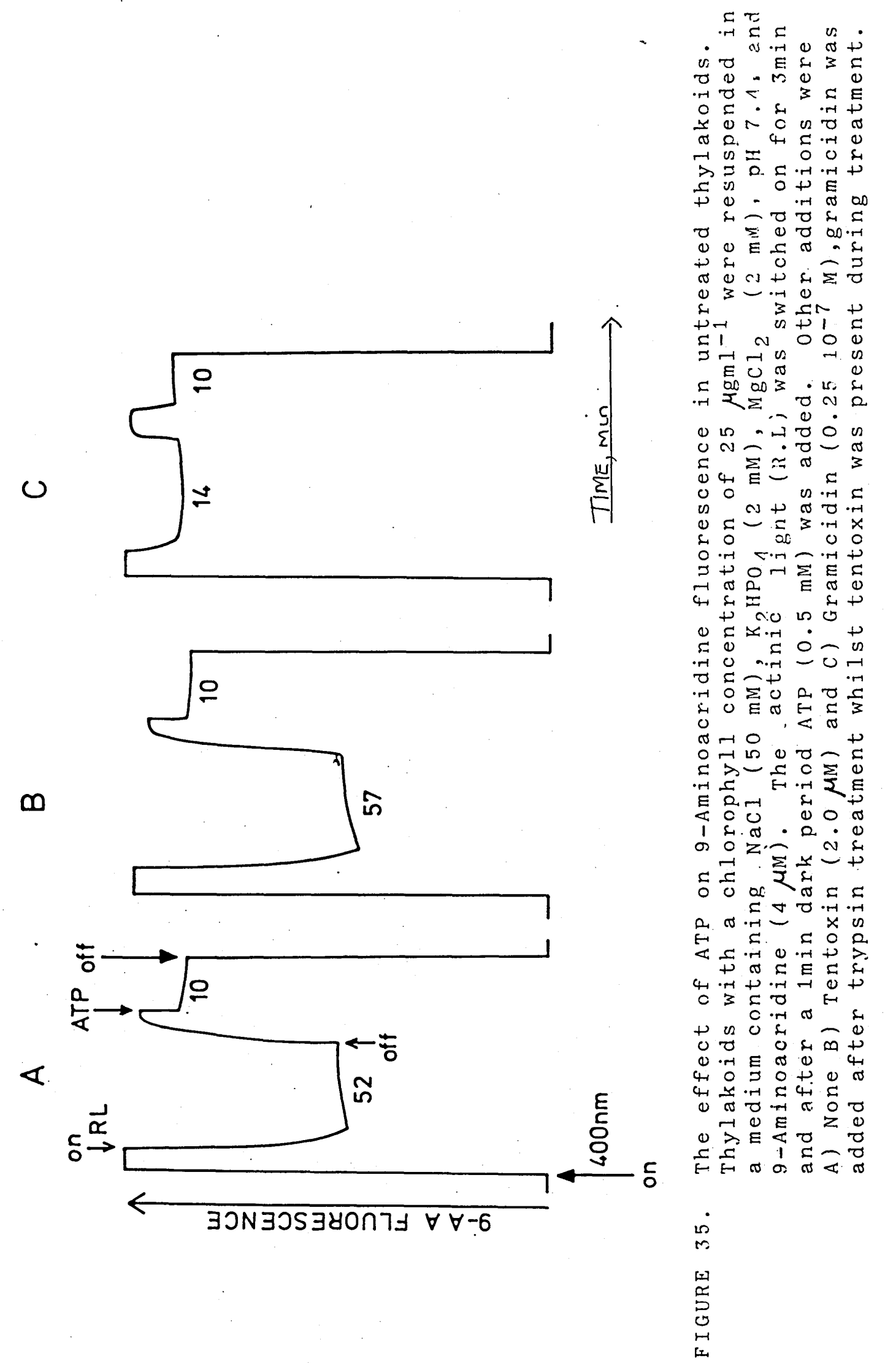


and is not related to the functional state of the membrane (and Schuurmans and Kraayenhof, 1983) Figures 36 and 37 show the effect of ATP on membranes which have been pretreated with trypsin. It can be seen that in dark-treated trypsin samples (Fig 36A) that addition of ATP caused a rapid decrease in the 9-Aminoacridine fluorescence intensity 1 evel and this decrease was insensitive to uncouplers (Fig 36(B)). However, when membranes were treated with trypsin in the light. (Fig. 37A) addition of ATP resulted in a slow decrease in the fluorescence, in addition to the fast decrease observed in the controls and dark-treated trypsin samples. Moreover, this slow decrease was sensitive to both gramicidin and tentoxin (Fig. 37 B and C).

The effects of ATP on chymotrypsin-treated sampies are shown in (Figs. 38 and 39): dark-treated chymotrypsin (Fig. 38A) samples gave the same results as the control and dark-treated trypsin samples, in that addition of ATP caused a rapid, uncouplerand tentoxin - insensitive decrease in 9-Aminoacridine fluorescence (Fig 38 B and C). But, like trypsin, the addition of ATP to light-treated trypsin samples caused a slow, uncoupler-and tentoxinsensitive, decrease in fluorescence (Fig 39 ).

These results suggest that both trypsin and chymotrypsin altered the membranes in such a way that addition of ATP induced membrane energisation. The inhibition of this effect by gramicidin and tentoxin further suggests that trypsin and chymotrypsin activated ATP hydrolysis and it was the reverse proton-pumping activity of the ATPase which was responsible for membrane energisation. 


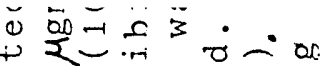

कर

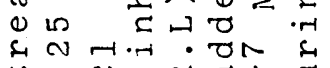

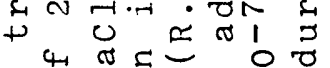

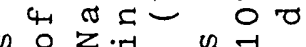

(1) $\quad \pi+\infty x+$

EE of a 3 L 3

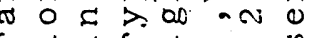

4.

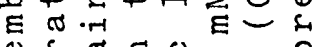

(1) म

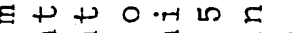

द E.HE . . n

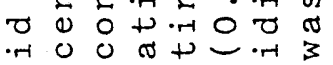

.

章

$\rightarrow$. $50<\pi$
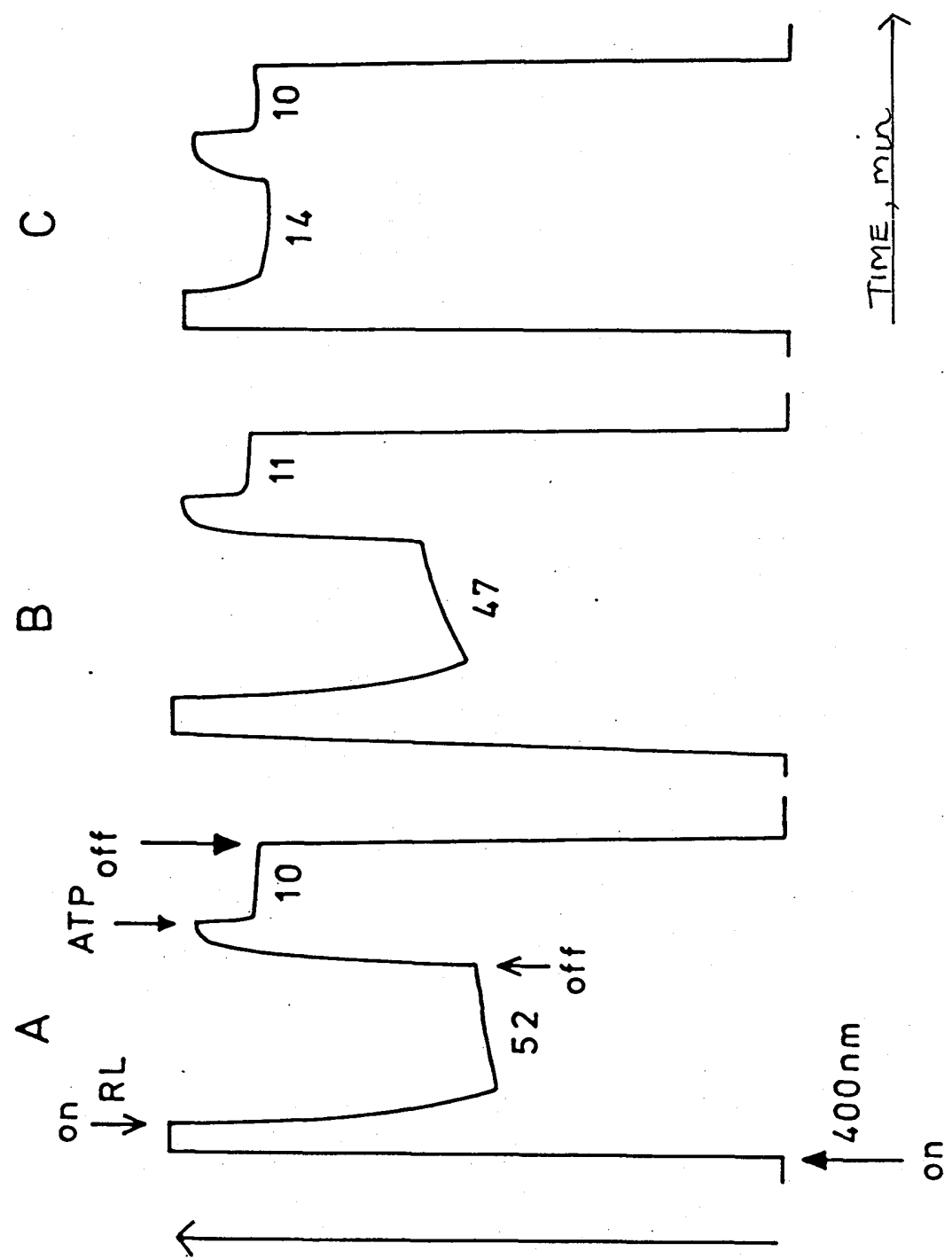

तन

हन

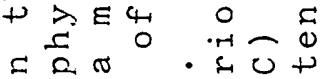

$\rightarrow C E \sim 0$

\& $5 \circ \sum 2 \cdots+$

(1) O $0 \cdot$.

$0 न+{ }_{0}$

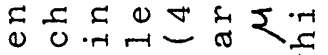

O 0 ह

थ त

( ) D

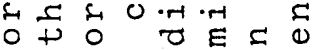

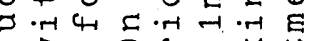

4 3 - $\begin{aligned} & 0 \\ & 4\end{aligned}$

जन त $0+0$

$001 \cdot 0+E \&$

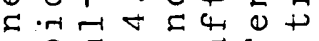

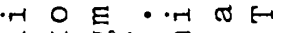

$O \rightleftharpoons$ ON

-

का

过

है

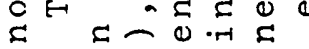

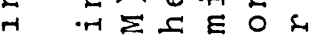

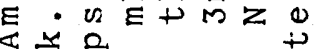

$1 \&>N$ \& $\rightarrow 4$

का $\pi$ क $00<\pi$

0 क 04

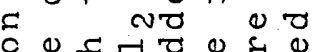

C 0 舟

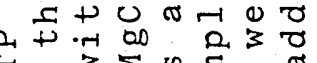

E $3 \sum n E$

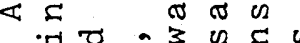

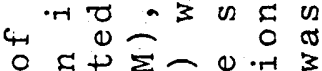

0 \&

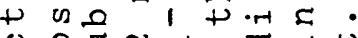

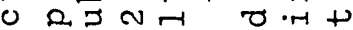

$0 \gg O-E E \sigma \nabla E$

4 \& E D C

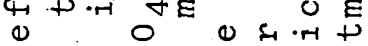

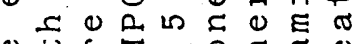

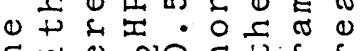

E

ヨวกヨวงヨช0กาป $\forall \forall-6$

$\dot{m}$

5
0
5
0
0
5
51 


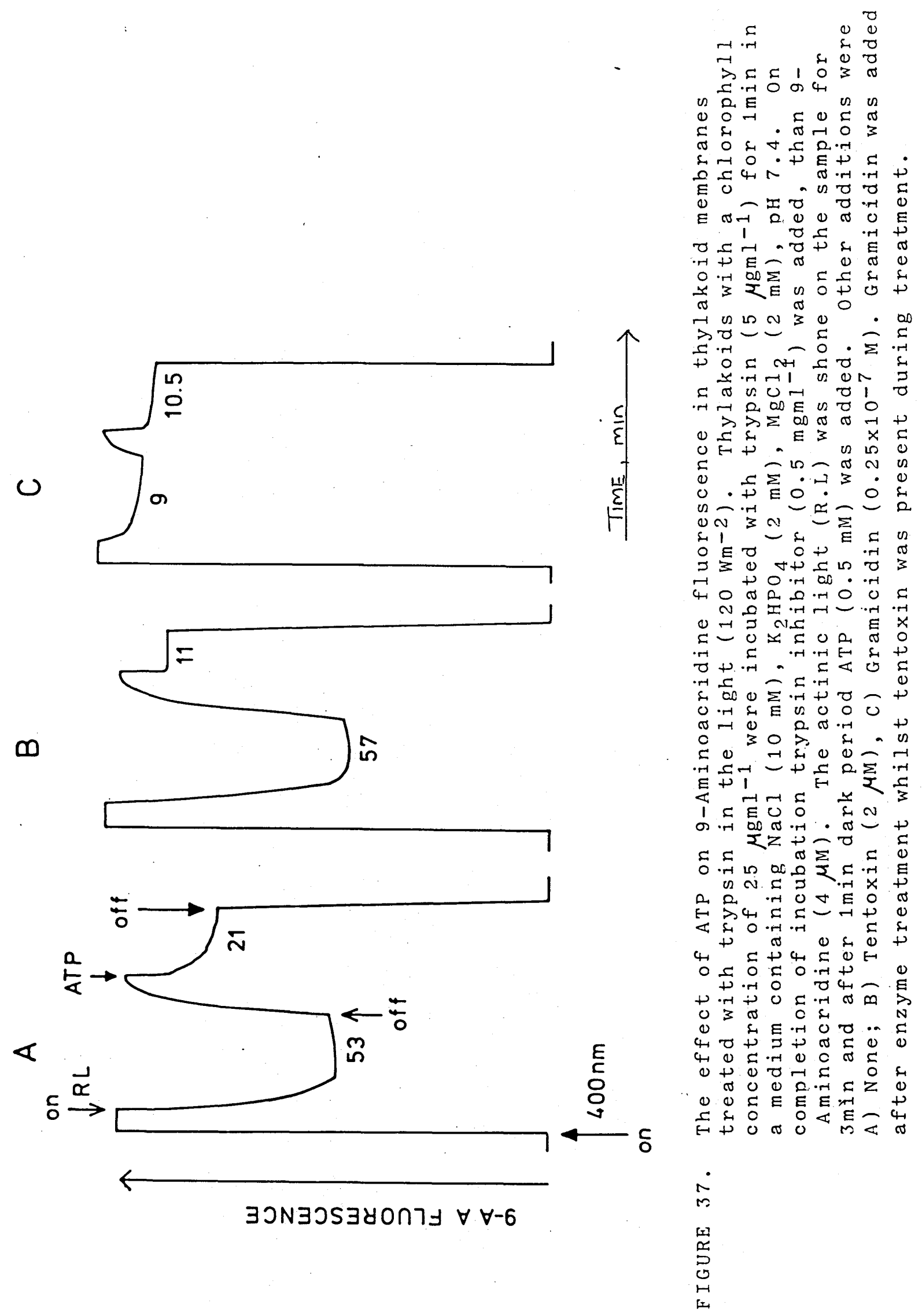



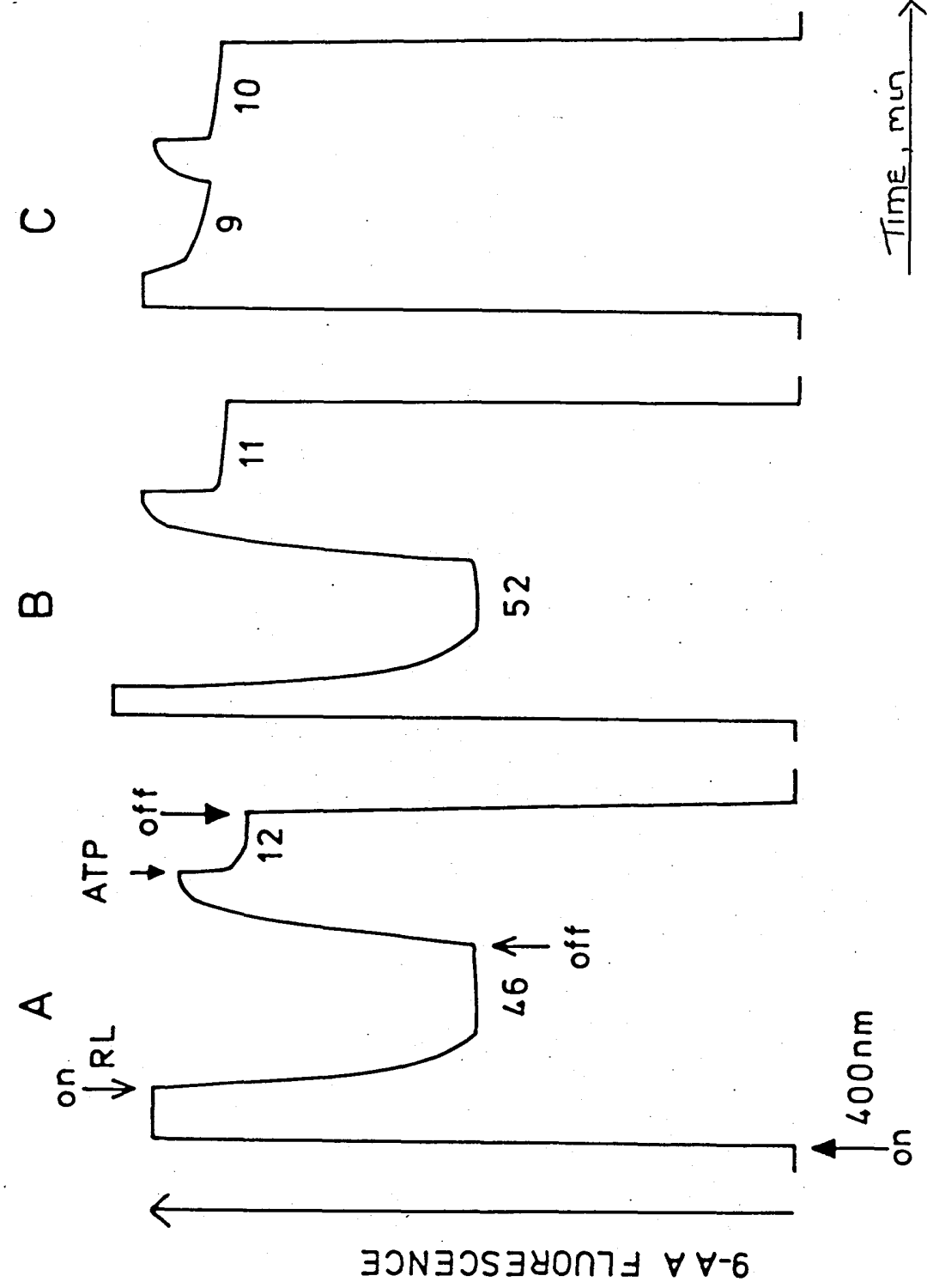

$\therefore \quad$ \&

\& 5 क ष 0

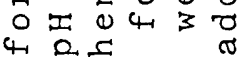
a

is $0 .-100$

c $\rightarrow-200$

ब

म $T$ E E 0 U

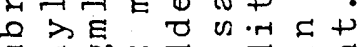

Eᄃ

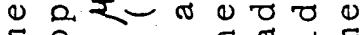

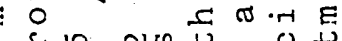

$0+$ व 0 न 正

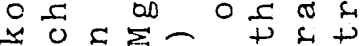
त) $r$ r 000 तो 02010

E

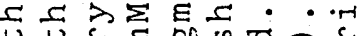
. [ 300 皮 . $m-L$ U

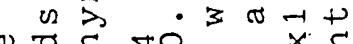
in $x$

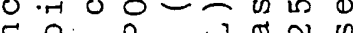
范年

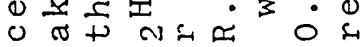
or $\rightarrow$.

$0>3+\sum$

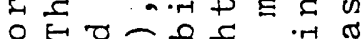

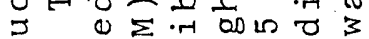

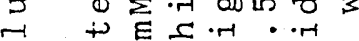
4 - क ह न 0 O

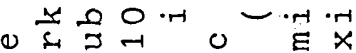

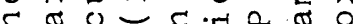
I 0 व मी

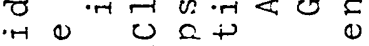
द E 0 ब O†

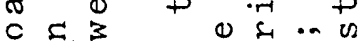

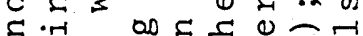

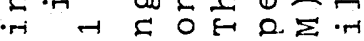
E $\Sigma \overrightarrow{1} \cdot \vec{r} \cdot \vec{r}$.

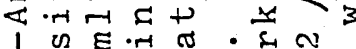

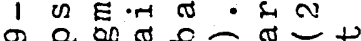

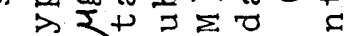
ह से $0 \sum^{2}$ \& क म

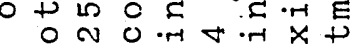
a. $E>$ E 4 H $\rightarrow 0$

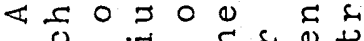

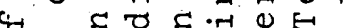
क- 10 ह

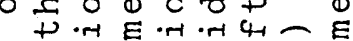

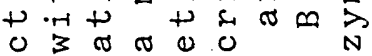

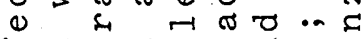

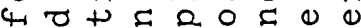

4-1 O E.H E ह ठ 5 $0+0$. $0 . r$ o ๙ O $=0 E E Z 0$ (1) 0 E $A$ H

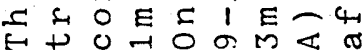

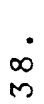




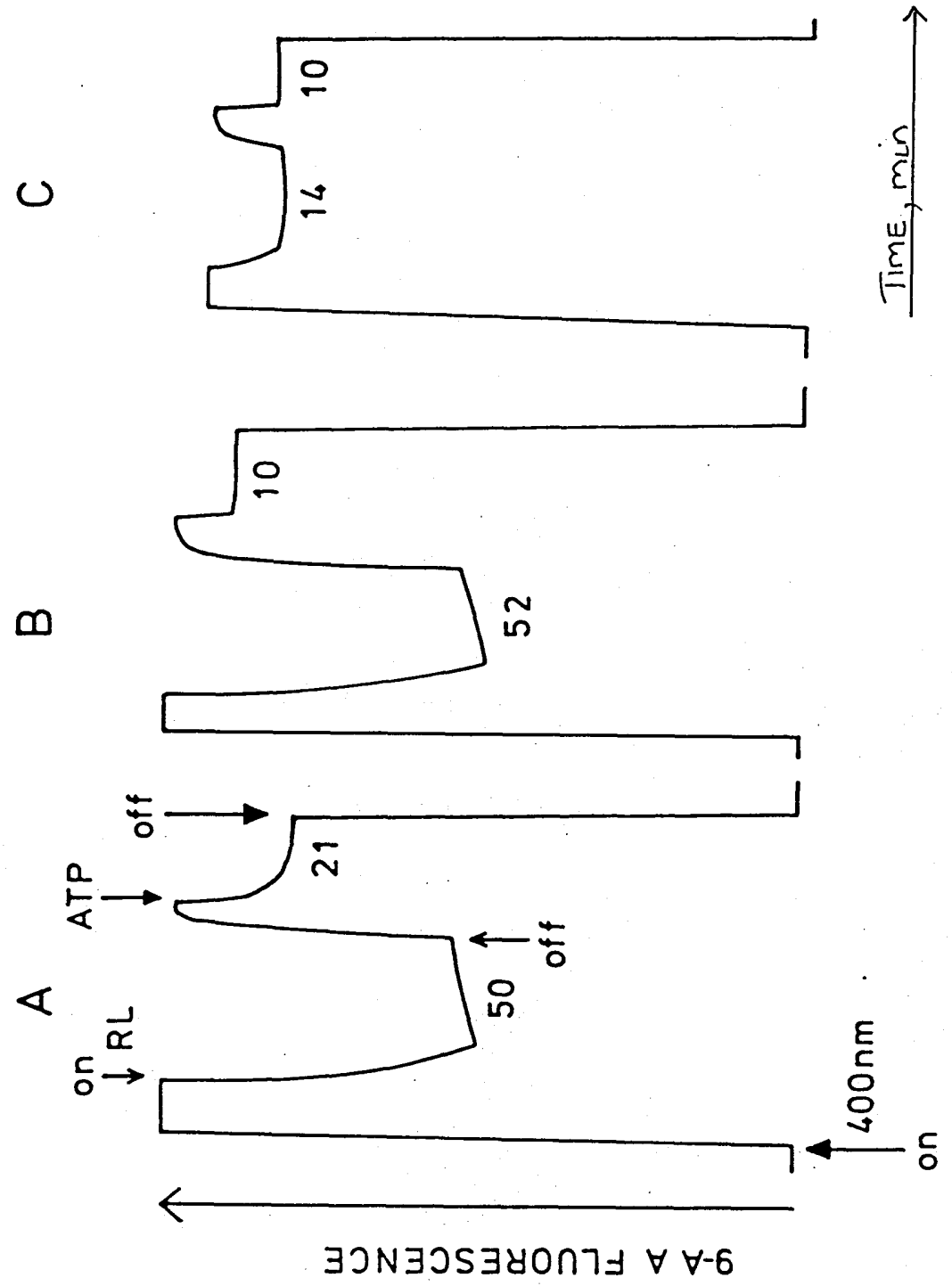

$\nabla 1 \cdot r s$

$\sim m z s$

N $N M Z \frac{1}{0}$

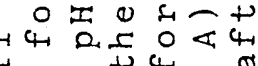

0 10 का

Eन $=$ - 1 \&

$\infty 01 \sum 0+100$

\& 0 E 0030

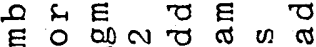

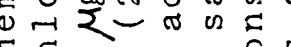

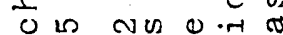

ठ $\rightarrow$ का त 3

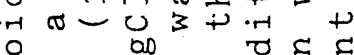

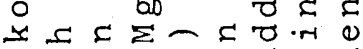

बक त

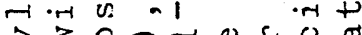

$\leq E D$ \&

(2)

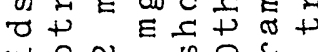

E.H ON NO A

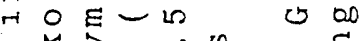

ते

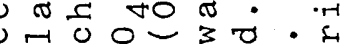

$\Rightarrow \rightarrow 000$

$\overrightarrow{2}$ 도 $-\frac{0}{0} \overrightarrow{0}$

0 E $N$ N $\rightarrow$ O

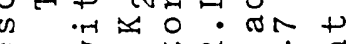

1) $3+4 \Sigma$ a 11

$0 \div \overline{0} 0$ o 0 - 0

उN $0 \sum \cdot+1+3.0$

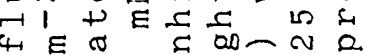

$\sum 00 \cdot n \cdot r \sum \sum$

(1) J $\mathrm{C}$ E $\mathrm{O}$ U

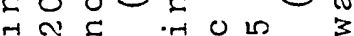

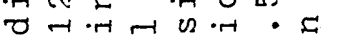

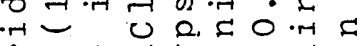

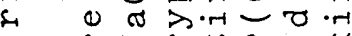

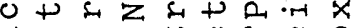

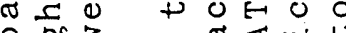

0 00 $3 \quad$ क $<\cdot 4+$

L.H OLE E

न नન Д 0 व

E 1 .

1 工 $E \cdot 4$ क

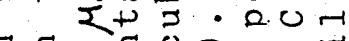

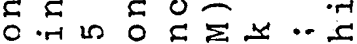

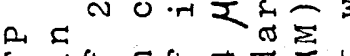

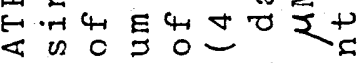
o.

$4 \Rightarrow E \sigma E \cdot T-E$

- L $O$ U $O$ E E H

H.H E.H.

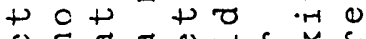

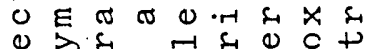

4 C

4 O E.H E त फ E 1

0 O C 0 त 0 ह

त 0 工 0 E $0+$ E. I .

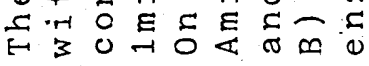

0
$m$
0
0
0
0
0
0
1 
42.8 The effect of trypsin and chymotrypsin on the sub-units of the coupling factor.

The effects of trypsin and chymotrypsin on the CF sub-units were investigated using polyacrylamide gel electrophoresis (PAGE). Figures 40 and 42 show the results of a 5 min dark incubation of thylakoids with trypsin or chymotrypsin. Lanes 1 and 8 (Fig 40) show the four standards which are bovine serum albumin ( $68 \mathrm{KDa})$, alcohol dehydrogenase $(43 \mathrm{KDa})$, myoglobin $(17 \mathrm{KDa})$ and cytochrome $\underline{\mathrm{c}}$ $(12.4 \mathrm{KDa})$. The untreated $\mathrm{CF}_{1}$ is depicted in Lanes 9 and 10 and the five sub-units corresponding to the polypeptides of $\mathrm{CF}_{1}$ can be seen; these sub-units are a $(59 \mathrm{KDa}), \boldsymbol{\beta}(56 \mathrm{KDa}), \gamma(37 \mathrm{KDa}), \delta(17.5 \mathrm{KDa})$ and $\varepsilon(13.5 \mathrm{KDa})$. Looking next at Lanes 5-7, these show the effect of 5 min trypsin treatment in the dark on the sub-units of $\mathrm{CF}_{1}$. It can be seen that the five bands are essentially the same as seen in the control (Lanes 9 and 10 ). On the other hand chymotrypsin incubation in the dark (Lanes 2 -4) appears to affect the $\beta$ sub-unit in that a third band not previously visible can now be seen. Moreover the $\boldsymbol{\beta}$ band is much lighter than in the controls (Lanes 9-10) or similarly treated trypsin samples (Lanes 2-4). Figure 42 shows the region of the $\boldsymbol{\alpha}$ and $\boldsymbol{\beta}$ bands in higher magnification. The effect of trypsin and chymotrypsin incubation in the 1 ight is shown in Figures 41 and 43 . Lanes 2-4 in Figure 41 show the effect of trypsin and it can be seen that there is a hint of a third band lying below the $\boldsymbol{\beta}$ sub-unit. In the case of chymotrypsin it can be seen here, as in the dark treated samples, that the

$\boldsymbol{\beta}$ sub-unit appears to split into two bands. The results presented here although preliminary at best suggest that chymotrypsin (in either the light or dark) and trypsin in the light affected the $\beta$ sub-ynit. 


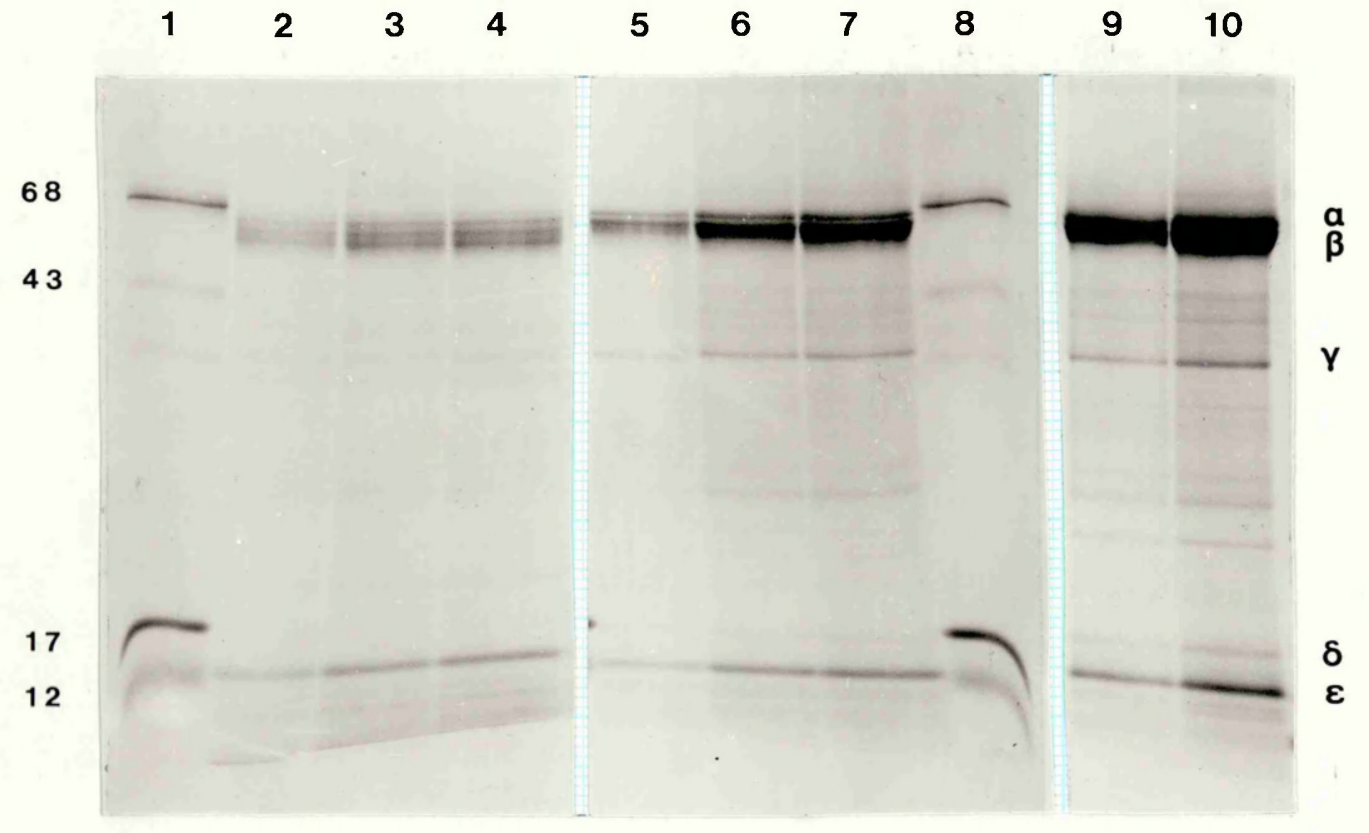

FIGURE 40 .

Polyacrylamide gel electrophoresis of $\mathrm{CF}_{1}$ extracted from thylakoids treated in the dark with trypsin or chymotrypsin. Thylakoids with a chlorophyl1 concentration of $25 \mu \mathrm{g} \mathrm{ml^{-1 }}$ were incubated with trypsin ( $5 \mu \mathrm{g} \mathrm{m1^{-1 }}$ ) were incubated with trypsin ( $5 \mu \mathrm{ml}^{-1}$ ) or chymotrypsin $\left(15 \mu \mathrm{m} \mathrm{m}^{-1}\right)$ in a medium containing Tricine $(20 \mathrm{mM}), \mathrm{KCl}(10 \mathrm{mM})$ and $\mathrm{MgCl}_{2}$ (2 $\left.\mathrm{mM}\right) \mathrm{pH} 7.4$. The reaction was stopped by adding trypsin inhibitor $\left(0.5 \mathrm{mg} \mathrm{m1^{-1 }}\right)$ Lanes 1 and 8 are standards; 2-4 chymotrypsin treated; 5-7 trypsin treated and 9 and 10 are untreated controls. This was a gradient gel $(12-15 \%)$ and the loading of protein was in the range of $10-25 \mu g$.

Frotedn loated: Lanes 2 and $5,10 \mu$; lanes 3 and $6,15 \mu \mathrm{g}$;

Janes 4 and $7,25 \mu$; lane $9,25 \mu \mathrm{g}$; lane $10,50 \mu \mathrm{g}$. 


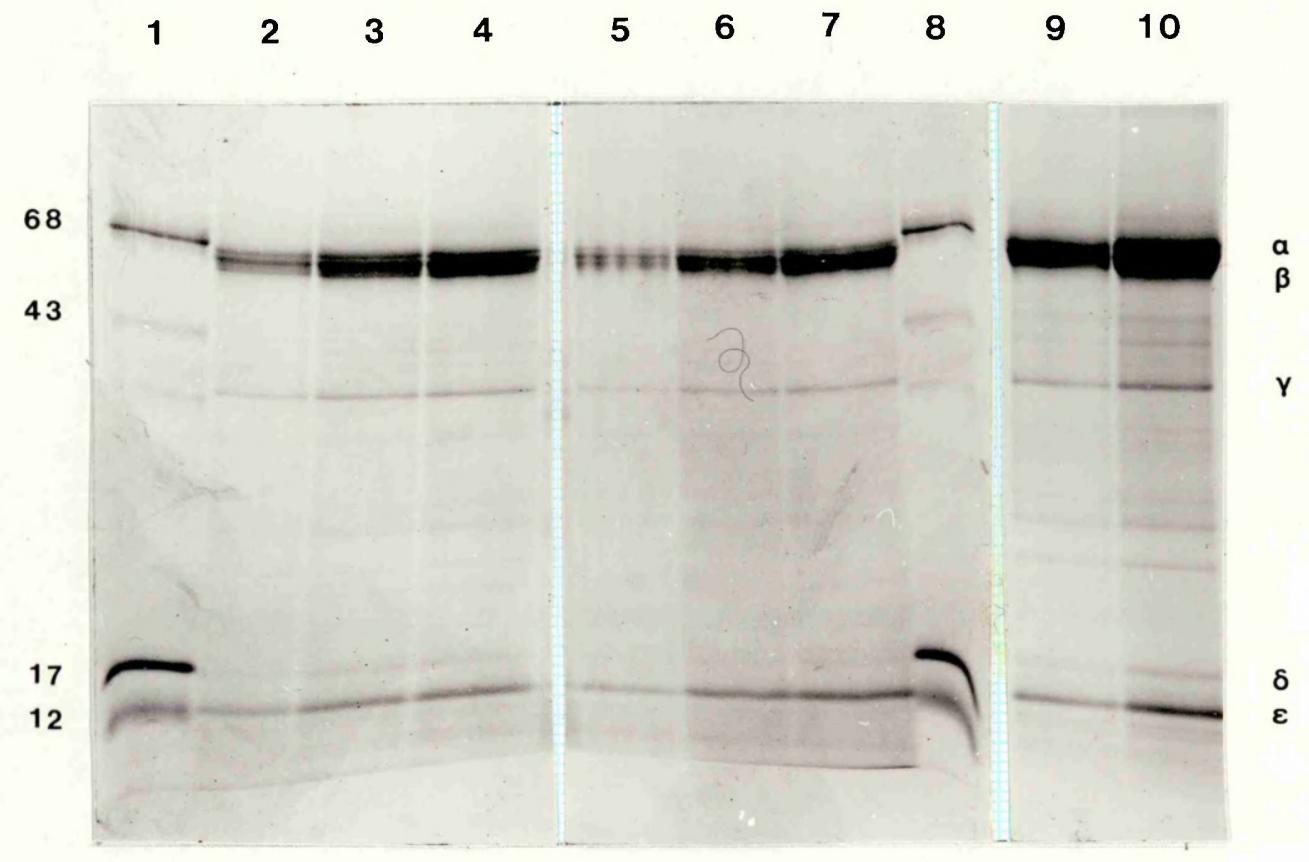

FIGURE 41 .

Polyacrylamide gel electrophoresis of $\mathrm{CF}_{1}$ extracted from thylakoids treated in the light with trypsin or chymotrypsin. Thylakoids with a chlorophyll concentration of $25 \mu \mathrm{g} \mathrm{ml^{-1 }}$ were incubated with trypsin

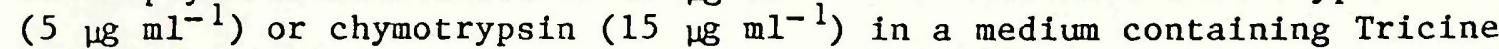
$(20 \mathrm{mM}), \mathrm{KCl}(10 \mathrm{mM})$ and $\mathrm{MgCl}_{2}(2 \mathrm{mM}) \mathrm{pH} 7.4$. The reaction was stopped by adding trypsin inhibitor ( $0.5 \mathrm{mg} \mathrm{ml}^{-1}$ ) Lanes 1 and 8 are standards; 2-4 trypsin treated; 5-7 chymotrypsin treated and 9 and 10 are untreated controls. This was a gradient gel (12-15\%) and the loading of protein was in the range of 10-25 $\mu \mathrm{g}$.

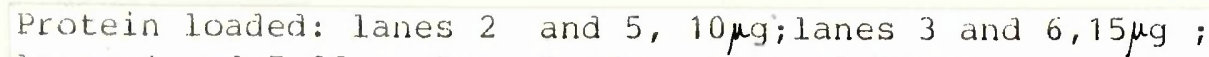

lanes 4 and $7,25 \mu \mathrm{g}$; lane $9,25 \mu \mathrm{g}$; lane $10,50 \mu \mathrm{g}$. 


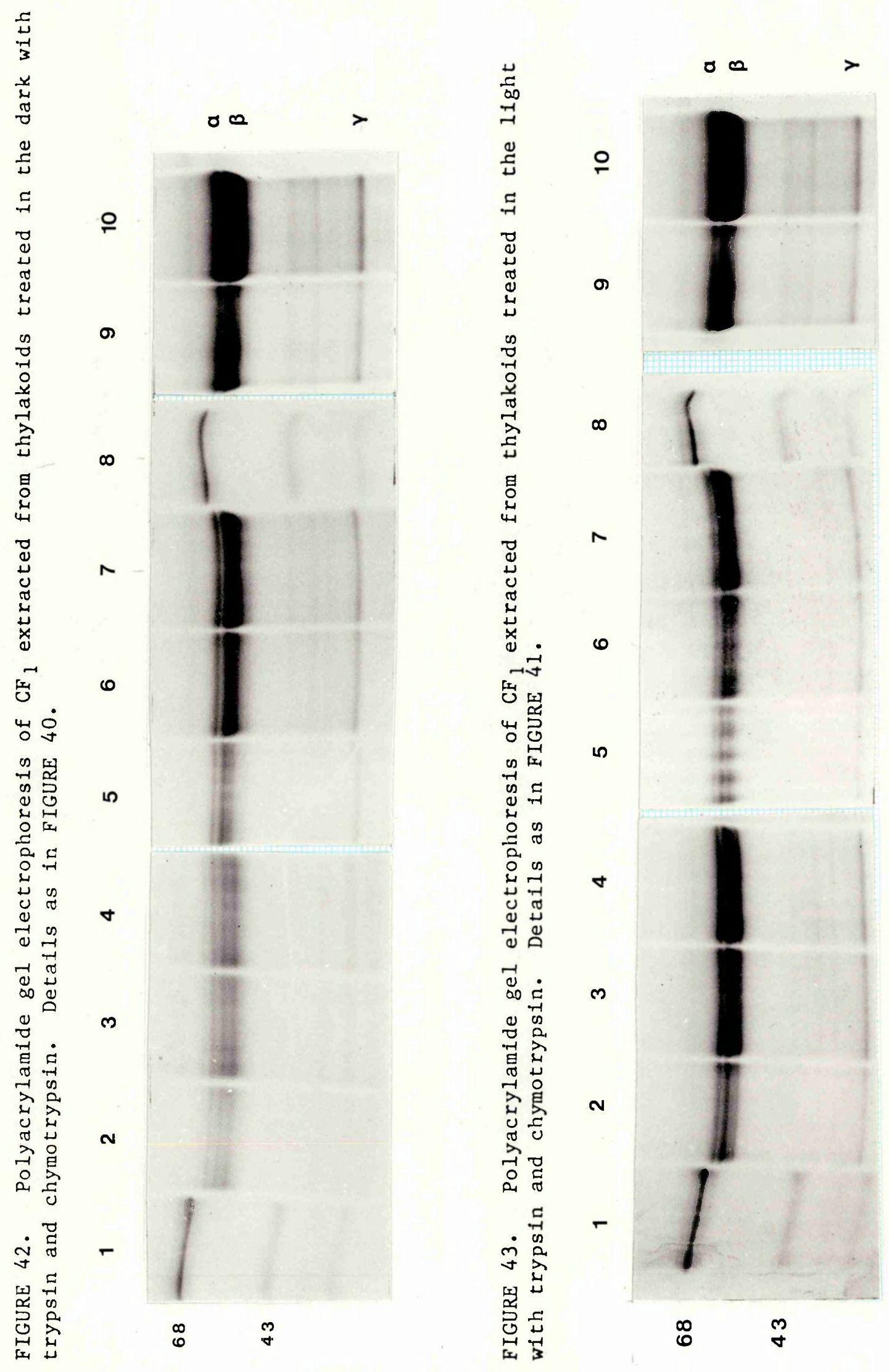




\subsection{DISCUSSTON.}

4.3.1 Uncoupling of the thylakoid membrane caused by trypsin and chymotrypsin treatments.

It was showr in chapter 3 (Figs 18 and 19) that trypsin treatment, and to a lesser extent chymotrypsin treatment, stimulated the rate of electron transport (water to ferricyanide.). This effect was more marked when the enzyme (either trypsin or chymotrypsin) incubation was carried out in the light. These data suggested that trypsin and chymotrypsin may uncouple thylakoid membranes. In this chapter, trypsin and chymotrypsin were shown to decrease the half-time of the decay of the electrochromic bandshift; this is indicative of an increase in the ionic permeability of the membrane and supports the suggestion that these proteolytic enzymes uncouple the membrane. Here again it was noted that trypsin had a more marked effect than chymotrypsin. Moreover, the effect of trypsin treatment in the light on the electrochromic bandshift was greater than for similar dark treatment; this was not the case for chymotrypsin, where little difference was noted between 1 ight- and darktreated samples.

Further support for the uncoupling hypothesis came from measurements of phosphorylation rates. These results showed ( $\mathrm{T}$ ables 7 and 8 ) that both trypsin and chymotrypsin inhibited phosphorylation. In dark-treated membranes trypsin and chymotrypsin had comparable effects. In 1ighttreated membranes the inhibition was more marked, and trypsin had a much greater effect, with total inhibition of phosphorylation occurring after $5 \mathrm{~min}$. 
Another difference which was noted between the effects of trypsin and chymotrypsin was that after $1 \mathrm{~min}$ lighttreatment with trypsin a stimulation of phosphorylation (and oxygen evolution) occurred; this was not seen in the case of chymotrypsin. Moreover measurements of oxygen evolution (water to methyl viologen) were made simultaneously with phosphorylation and these showed that both enzymes caused some inhibition of electron transport. In the case of trypsin this inhibition was not very significant and was probably not a contributary factor in the inhibition of phosophorylation. However, chymotrypsin had a more marked effect on electron transport than trypsin and this may be at least partially responsible for the decrease in the rate of phosphorylation observed after chymotrypsin treatment.** SEE FOOTNOTE OVERLEAF

\subsubsection{Trypsin and chymotrypsin: effects on ATP

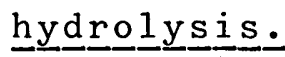

Treatment of thylakoids in the light with dithiothreitol, trypsin and chymotrypsin altered the membrane in such a way that addition of ATP caused a marked decrease in the half-time of decay of the electrochromic bandshift (Figs. 30, 31 and 32 ). Dithiothreitol is known to activate the ATPase hydrolytic (Petrack et al , 1965) therefore the decrease in the half-time of decay of the bandshift caused by addition of ATP to these samples may reflect the presence of this reaction. Since addition of ATP to trypsin- and chymotrypsintreated samples induced a similar change as was seen with DTT this suggested that ATP hydrolysis was occurring in these membranes. Tentoxin, which is an inhibitor of ATP hydrolysis, inhibited the effect of ATP on membranes treated with dithiothreitol, trypsin or chymotrypsin in the light. These results, together with measurements made of hydrolysis rates in similarly treated thylakoids, supported the proposal 
* * Footnote

It is worth noting that the results in this chapter have shown that the activation of the ATPase complex precedes uncoupling of phosphorylation. In addition it can be seen that trypsin stimulated the rate of phosphorylation and the rate of electron transport under conditions which induced the activation of ATP hydrolysis. This result is important in relation to the proposal made by Mills and Mitchell(1984)that thiol modulation of the ATPase complex emables the enzyme to become active over a lower range of pH. 
that trypsin and chymotrypsin were activating the ATP hydrolase. One model for describing the effect of ATP on the electrochromic bandshift of thylakoids with an active ATP hydrolase concerns hydrolysis of ATP in the dark which would cause reverse proton pumping (Carmeli, 1970) resulting in the build up of a pH gradient across the membrane: This effect increases the total $\Delta \mu_{H^{+}}$and the steady-state driving force across the thylakoids which would then induce the 1 ight-induced field to decay at a greater rate (c:f: Siggel, 19'84).

This model predicts that in the dark a $\Delta \mathrm{pH}$ will build up across the thylakoids. Using 9-Aminoacridine fluroescence it was shown that, in membranes pretreated in the light with trypsin or chymotrypsin, ATP induced the formation of a $\Delta p H$. This result supports the model proposed above.

\section{3 .3 The ATPas}

The coupling factor $\left(\mathrm{CF}_{1}\right)$ of the ATPase complex is composed of 5 sub-units called $\boldsymbol{\alpha}, \boldsymbol{\beta}, \boldsymbol{\gamma}, \boldsymbol{\delta}$ and $\boldsymbol{\varepsilon}$ in order of descending molecular weight (see section 1.5). Each of these sub-units has been assigned a different function: the $\alpha$ and $\beta$ subunits contain the regulatory and active sites respectively and are therefore thought to be directly involved in catalysis; the $Y$ sub-unit is believed to control the flow of protons through the complex; the $\delta$ sub-unit binds $C_{1}$ to the $\mathrm{CF}_{0}$ portion of the ATPase in the membrane; and the $\boldsymbol{\varepsilon}$ sub-unit is believed to have an inhibitory role al though this is not unequivocal. 
The results in this chapter have shown that both trypsin and chymotrypsin affect the functions of the ATPase complex in two ways: 1) phosphorylation is inhibited and 2) hydrolysis is stimulated. These effects are most likely caused by partial digestion of sensitive sites on one or more of the sub-units of $\mathrm{CF}_{1}$ : two of the sub-units have been proposed as candidates for digestion by trypsin and these are the $\gamma$ sub-unit (Moroney and McCarty, 1982) and the $\varepsilon$ sub-unit (Nelson et al, 1972). Comparing the effects of trypsin and chymotrypsin it appears that chymotrypsin had a similar but less marked effect than trypsin. However, examining the data more closely it can be seen that this is not the case because 1) the electrochromic bandshift data show that trypsin has a more marked effect on lighttreated samples than similar dark-treated membranes. But with chymotrypsin the half-time of decay of the bandshift was slightly longer in the light- treated membranes than in similar dark- treated ones; and 2) the phosphorylation data shows that, after $1 \mathrm{~min}$ incubation in the light with trypsin, the rates of phosphorylation and oxygen evolution were significantly stimulated. In chymotrypsin-treated samples there was no hint of stimulation under any of the conditions investigated. Therefore the effects of these enzymes on the functions of the ATPase complex are different and this may be reflected in the differences in the sensitive sites on the $\mathrm{CF}_{1}$. The $\varepsilon$ sub-unit of $\mathrm{CF}_{1}$ is believed to act as an inhibitor of ATPase function (Nelson et al 1,1972 and Finel et a, 1984); it is possible that the tryspin-induced stimulation of phosphorylation is due to an effect on the $\varepsilon$ sub-unit causing it to change position. However, Moroney and McCarty (1982) correlated a trypsininduced change in the $\gamma$ sub-unit with the activation 
of hydrolysis and the inhibition of phosphorylation. In addition they suggested that these effects of trypsin were dependent on incubation with the enzyme in the light.

PAGE analysis of $\mathrm{CF}_{1}$ presented here does not support either of these hypothesis and it appeared to be either the $\boldsymbol{a}$ or the $\boldsymbol{\beta}$ sub-units which were affected by both trypsin and chymotrypsin in the light. In the dark-treated samples a change in the $a$ or $\beta$ sub-unit was caused by chymotrypsin but not by trypsin. Moreover inhibition of phosphorylation by trypsin or chymotrypsin was not strictly light- dependent but stimulation of ATP hydrolysis was : this suggests that two different sites may be involved in the control or catalysis of these reactions.

\subsubsection{The sensitivity of the electrochromic bandshift.}

The sensitivity of the electrochromic bandshift, to treatment which alters the membrane integrity or reduces the efficiency of one or both photosystems, has been known for some time (see Junge, 1977). During this investigation it was shown that the electrochromic bandshift was very sensitive to factors which alter the functional state of the ATPase enzyme complex. In Figure 24 it was shown that incubation of thylakoids in strong white 1 ight $\left(120 \mathrm{vm}^{-2}\right)$ changed the characteristics of the electrochromic bandshift: the slow rise phase was lost and the halftime of decay was decreased. This effect was not an allor-nothing effect and increasing the light incubation time resulted in a more makred change in the electrochromic bandshift signal. It is known that light-induced membrane energisation induces conformational changes in 
the ATPase complex which in turn alters the permeability of the membrane to protons (Magnusson and McCarty, 1976); this could in part explain the lightinduced decrease in the half-time decay of the bandshift. It was previously shown by Girault and Galmiche (1978) and Schuurmans et al (1981) that addition of either ATP or ADP to thylakoids resulted in a decrease in the half-time of the bandshift. Girault and Galmiche (1978) suggested that the effect of ATP and ADP was the same and was due to ATP hydrolysis (newly formed ATP in the case of added ADP) which increased the proton conductivity of the membrane, because of the $\Delta \mathrm{pH}$ formed during ATP hydrolysis. In experiments carried out here it was possible to separate the effects of ATP and ADP on the bandshift. Dithiothreitol, trypsin and chymotrypsin were shown to alter the functional state of the ATPase enzyme in thylakoid membranes treated in the light; addition of ATP to these membranes resulted in a marked decreased in the half-time decay of the electrochromic bandshift. However, addition of ATP and ADP to these membranes had no effect.

The effect of ATP on the bandshift may be caused by reverse proton pumping, brought about by hydrolysis of ATP (Carmeli, 1970), building up a large proton motive force resulting in a greater steady state $\boldsymbol{\Delta} \boldsymbol{\Psi}$. Hence, the flash-induced $\hat{\Delta} \dot{\psi}$ decays more rapidly to the new steady state: the rate of decay being proportional to the total $\boldsymbol{\Delta} \boldsymbol{\Psi}$ (c.f. Siggel, 1984). The most likely reason why ADP had no effect on the bandshift under the conditions looked at here, was because the flash length and repetition rate was not sufficient to allow a build up of a large enough membrane potential to activate phosphorylation. 
ГHAPTER FIVE - THE USE OF OXONOL VI AS A MEMBRANE PROBE TO INVESTIGATE THE EFFECTS OF TRYPSIN AND CHYMOTRYPSIN ON THE ELECTROCHROMIC BAINDSHIFT INDICATING =PIGMENTS. 
The use of oxono? VI as a membrane probe to investigate the effects of trypsin and chymotrypsin on the electrochromic bandshift indicating-pigments.

\subsection{INTRODUCTION.}

Incubation of thylakoids in the presence of trypsin or chymotrypsin was shown to have two effects on the electrochromic bandshift (chapter 4): 1) the half-time of decay was decreased and this has been correlated with changes in the function of the ATPase complex; 2) the amplitude was decreased. This decrease in amplitude could be due to a number of factors including: 1) a loss of pigments which sense the electrochromic bandshift; 2) a loss of a portion of the light harvesting complex (LHCP) responsible for energy capture and subsequent transfer to the reaction centres; and 3 ) a reduction in efficiency in one or both of the photosystems by a) a direct effect on the reaction centre proteins or b) a redistribution of energy between PSII and PSI which could result in an unequal contribution of each photosystem to the electrochromic bandshift.

The experiments in this section were designed to look at one of these possibilities; trypsin and chymotrypsin may digest the pigments which sense the bandshift thereby reducing the signal. In Rhodopseudomonas capsulata bacteria, after incubation of membranes in the presence of pronase, no change in the reaction centre signal occurred but a loss of the carotenoid shift was observed and this correlated with the loss of an absorption band of the antenna complex pigment (webster et a 1 1980). The chloroplast pigment-protein complexes are less well 
defined as compared to those of bacteria because of the complexity of the chloroplast system. It is not possible, as yet, in the chloroplast system to correlate particular pigment complexes with specific absorption bands. This has made it necessary to use a different method to try and elucidate the loss of absorption change in chloroplasts after enzymic digestion.

An extrinsic probe, oxonol VI, has been shown to be an indicator of the membrane potential in bacteria (Bashford et al, 1979b) and in chloroplasts (Galmiche and Giraul, 1981). (The theory of this probe is discussed in 1.9.1). The rationale for using oxonol VI is that it can be added to the thylakoid membranes after the completion of enzyme treatment, therefore the absorption shift of this dye will not be affected directly by the enzyme treatment. If trypsin and chymotrypsin were affecting the amplitude of the electrochromic bandshift by digesting the sensing pigments then the amplitude of the oxonol change should remain unaffected.

\subsection{RESULTS.}

5.2.1 The light-minus-dark difference spectrum of chloroplasts treated with oxonol VI.

Figure 44 shows the differerce spectrum, of chloroplasts treated with oxonol VI, in the wavelength range of 560$590 \mathrm{~nm}$. It can be seen that the maximum absorption change occurred around $575 \mathrm{~nm}$ therefore this wavelength was used in all subsequent measurements of the oxonol VI absorption change. 


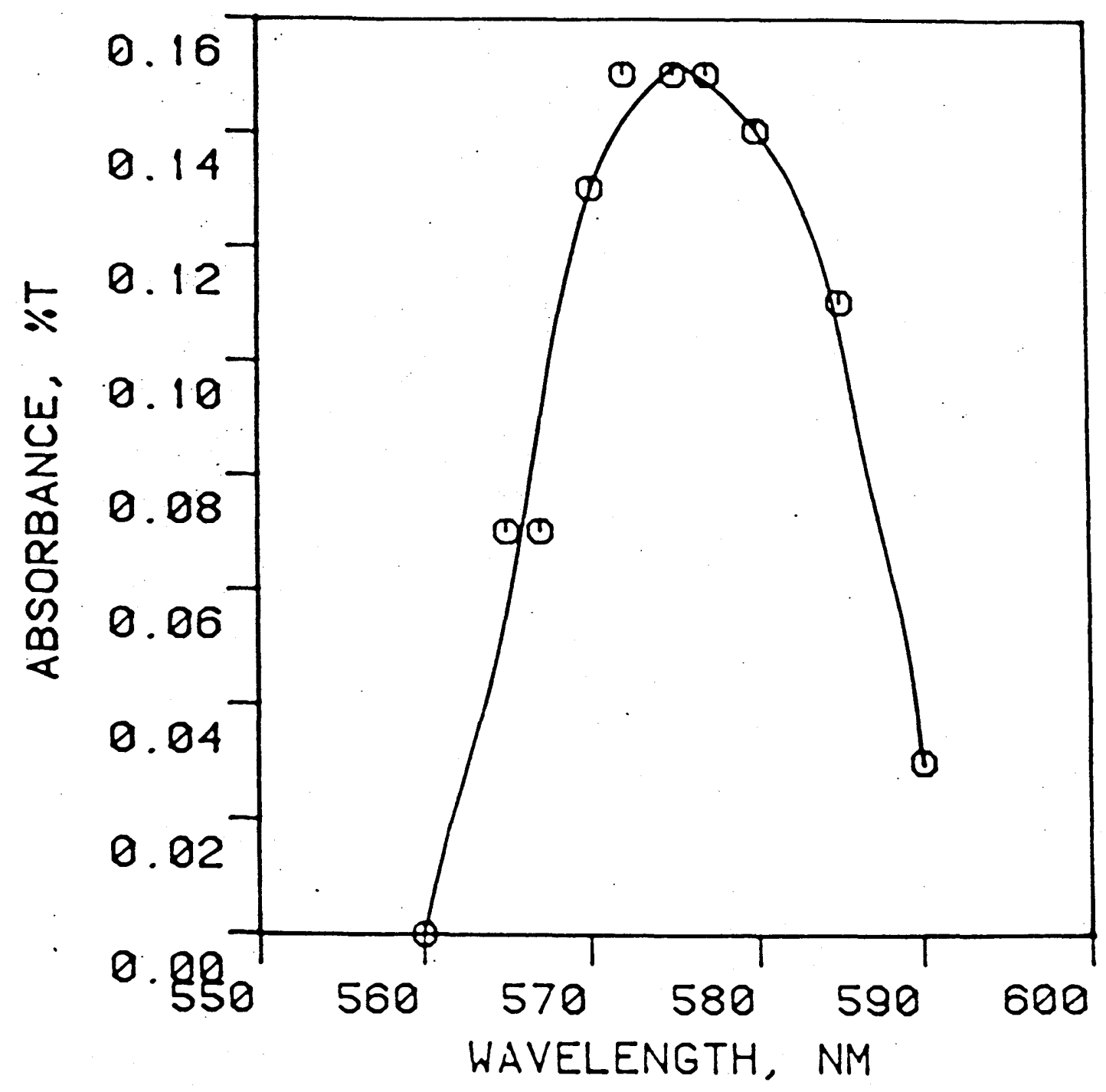

FIGURE 44 .

Light-minus-dark difference spectrum of chloroplasts in the presence of $2.0 \mu \mathrm{M}$ oxonol VI. The thylakoids with a chlorophyll concentration of $25 \mu g m 1^{-1}$ were suspendet in a medium containing $20 \mathrm{mM}$ Tricine, $10 \mathrm{mM} \mathrm{KCl}$ and $2 \mathrm{mM} \mathrm{MgCl} 2 \mathrm{pH} 7.4$. The signal obtained at each wavelength was the summation of 128 sweeps with a frequency of 1 per $5 \mathrm{~s}$. Each sweep was composed of a train of ' 6 flashes spaced $90 \cdot \mathrm{ms}$ apart. 


\section{2 .2 optimum oxonol vi con oncentration.}

oxonol VI has an uncoupling effect on thylakoid membranes as can be seen by its effect on the electrochromic bandshift: increasing the concentration resulted in a decrease in the half-time of decay of the bandshift signal (Fig. 45). For this reason it was important to choose an oxonol VI concentration which was sufficient to detect the oxonol VI absorption change ( $\triangle A 575)$, but yet did not have too high a concentration such that it dissipated the electric field that it was intended to measure. Figure 45 shows the effect of increasing oxonol VI concentration on the electrochromic bandshift and on the oxonol VI absorption change: the size of the oxonol VI signal increased as the concentration of oxonol VI was increased from 0.5-8.0 $\mu \mathrm{M}$. But looking at the electrochromic bandshift traces it can be seen that increasing oxonol VI concentration resulted in a decrease in the half-time decay of the bandshift showing that oxonol VI was dissipating the electric field. As a compromise a $1.0 \mu \mathrm{M}$ concentration of oxonol was used: this was a high enough concentration to give a measurable oxonol VI signal and the bandshift half-time of decay was reduced from $\sim 240 \mathrm{~ms}$ to $\sim 150 \mathrm{~ms}$.

5.2.3 The effects of trypsin and chymotrypsin on the oxonol VI absorption change and on the electrochromic bandshift.

Trypsin treatment in the dark was shown to decrease the half-time of decay of the electrochromic bandshift but, under these conditions, had little effect on the extent of this change (Fig. 46,1-4). However, under similar conditions the extent of the oxonol VI 


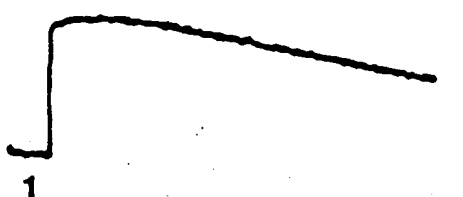

1
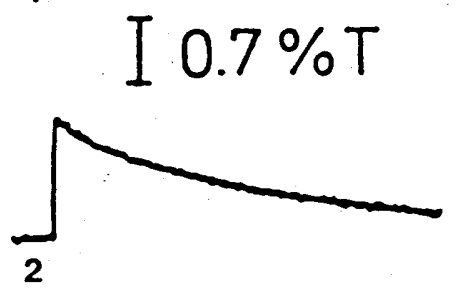

B

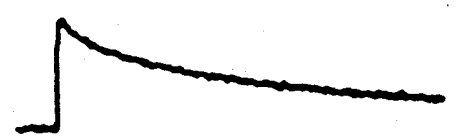

3

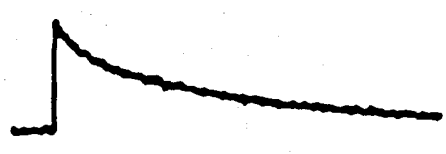

4
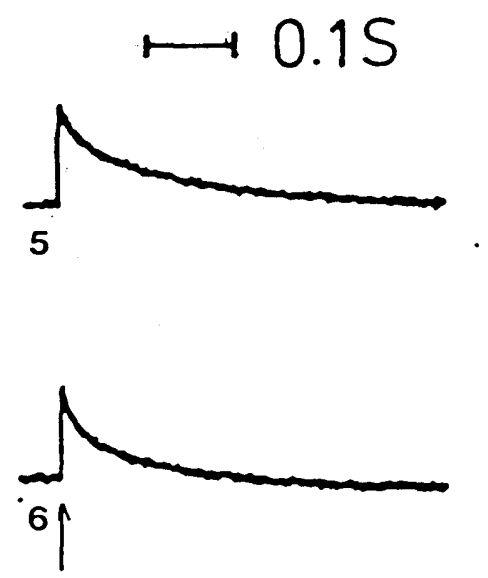

A

C

$F$
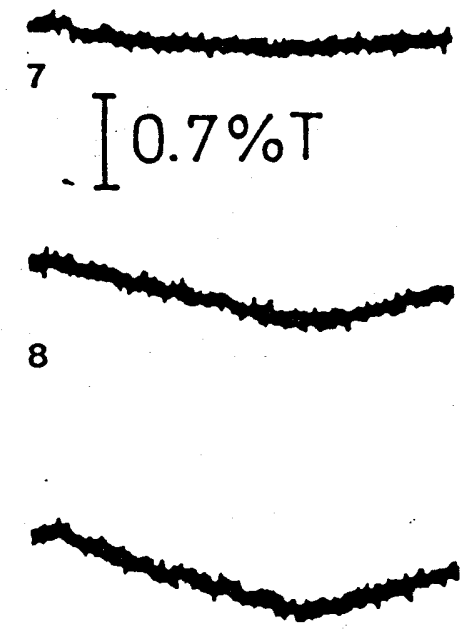

9

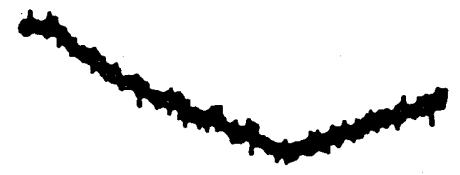

10

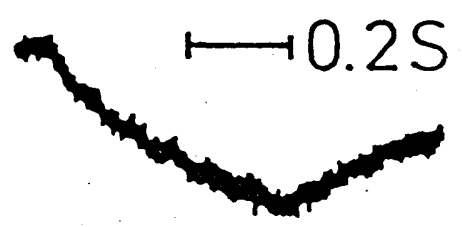

11

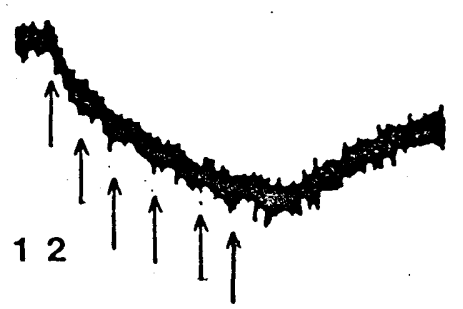

FIGURE 45 .

optimumum oxon ol VI concentration. The thylakoids with a chlorophyll concentration of $25 \mu \mathrm{gml}^{-1}$ were suspended in a medium containing $20 \mathrm{mM}$ Tricine, $10 \mathrm{mM} \mathrm{KCl}$ and $2 \mathrm{mM} \mathrm{MgCl}_{2} \mathrm{pH} 7.4$. The electrochromic bandshift signals $(1-6)$ were the summation of 64 single flash sweeps having a frequency of $1 \mathrm{~s}^{-1}$. Whilst the oxonol VI signals (7-12) were the summation of 128 sweeps fired at a frequency of 1 per $5 \mathrm{~s}$. Each sweep was composed of a train of 6 flashes spaced $90 \mathrm{~ms}$. apart. Oxonol VI concentration: A) Zero; B) $0.5 \mu M$; C) $1.0 \mu M$, D) $2.0 \mu M$; E) $4.0 \mu M$; F) $8.0 \mu \mathrm{M}$. 
absorption change was almost completely inhibited (Fig.46, 5-8). Similar results were obtained for light-treated trypsin samples (Fig. 47) with the effects being slightly more marked. Chymotrypsin caused the same changes in both the electrochromic bandshift and oxonol VI absorption change as trypsin but in this case there appeared to be little difference between the dark-(Fig.48) and the light-(Fig.49) treated samples.

It has been noted previously that the loxonol. VI response is much slower than the electrochromic bandshift (Galmiche and Girault, 1981) and this may be the limiting factor in these experiments. The rate of decay of the electrochromic bandshift wasstimulated slightly whilst the extent was only marginally reduced by these proteolytic enzymes yet the extent of the oxonol VI signal was almost completely inhibjted (Figs.46-49). It is possible that the half-time decay rate of the membrane potential is faster than the response time of oxonol VI.

5.2.4 A comparision between the effects of gramicidin and DCMU on the electrochromic bandshift and on the oxonol VI absorption change.

The effect of DCMU on the electrochromic bandshift and on the oxonol VI absorption change is shown in Figure50. It can be seen that the extent of the electrochromic bandshift was reduced by about $50 \%$ with DCMU $\left(7.5 \times 10^{-7} \mathrm{M}\right)$ whilst under the same conditions the oxonol VI absorption change was almost completely inhibited. The amplitude of the electrochromic bandshift is believed to indicate the magnitude of the membrane potential sreated by charge separation at both photosystems. Since the oxonol VI absorption change 


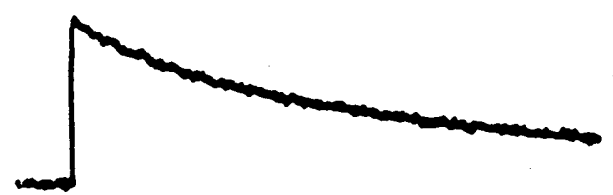

1

$\lceil 0.07 \%$ T

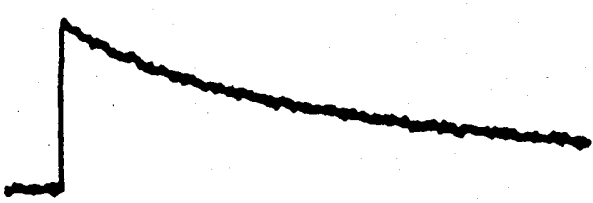

2

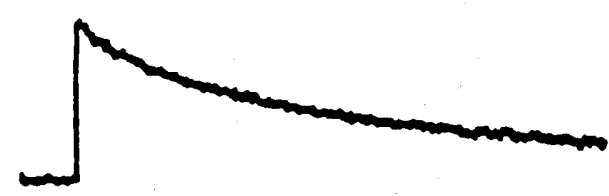

3
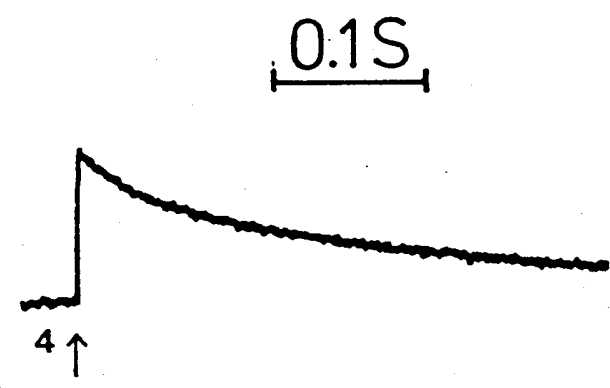

A

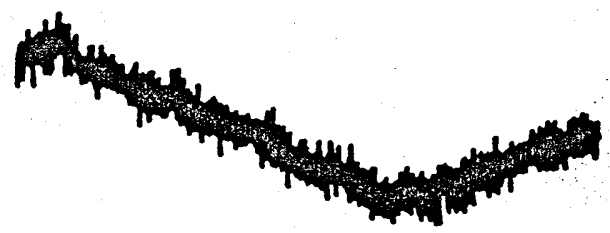

5
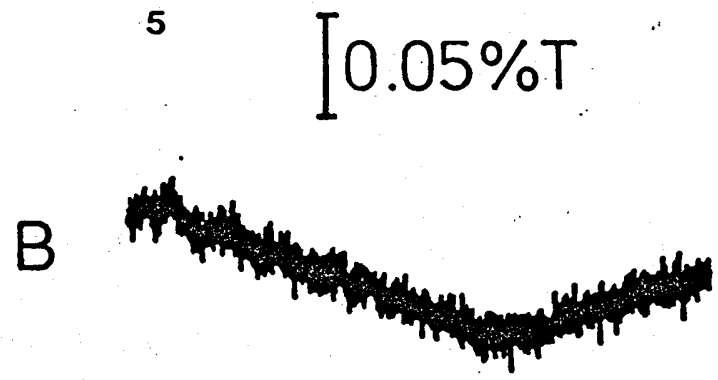

6

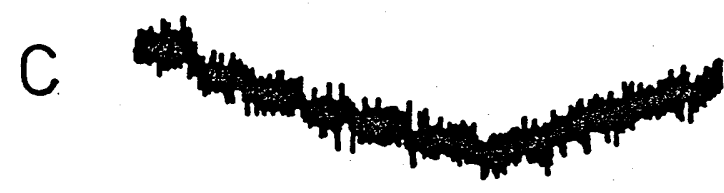

7
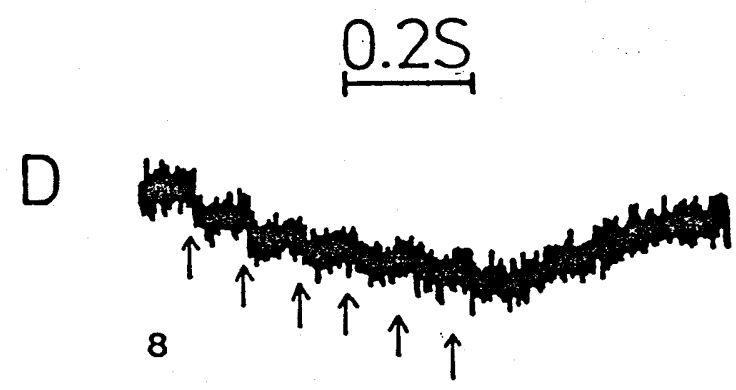

FIGURE 46 .

The effect of trypsin treatment in the dark on the electrochromic bandshift and the oxonol VI absorption change. Thylakoids with a chlorophyll concentration of $25 \mathrm{Mgml}^{-1}$ were incubated with trypsin $\left(1 \mu g m 1^{-1}\right)$ in the dark in a medium containing $20 \mathrm{mM}$ Tricine, $10 \mathrm{mM}$ $\mathrm{KCl}$ and $2 \mathrm{mM} \mathrm{MgCl}, \mathrm{pH} 7.4$. The reaction was stopped by adding trypsin inhibitor $\left(0.5, \mathrm{mgml}^{-1}\right.$ ) followed by oxonol VI $(1.0 \mathrm{MM})$. The electrochromic bandshift signals $(1-4)$ were obtained by summing 64 single flash sweeps with a frequency of $1 \mathrm{~s}^{-1}$ whilst the oxonol VI signals (5-8) were the summation of 128 sweeps fired at a frequency of 1 per $5 \mathrm{~s}$ - each was composed of a train of 6 flashes spaced $90 \mathrm{~ms}$ apart. Trypsin incubation times were: A) Zero, B) $1 \mathrm{~min}$; C) $2 \mathrm{~min}$; and D) $3 \mathrm{~min}$. 


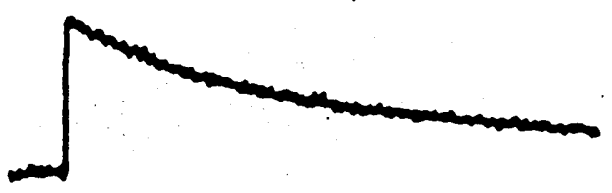

1

$\lceil 0.07 \% \mathrm{~T}$

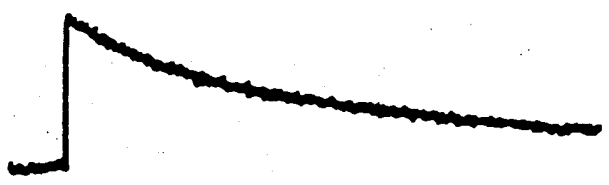

2

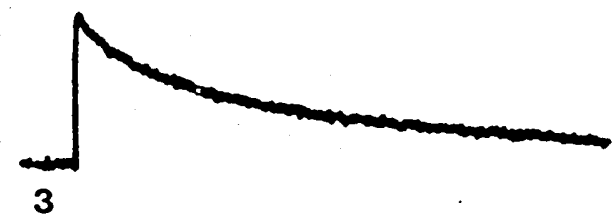

$0.1 \mathrm{~S}$

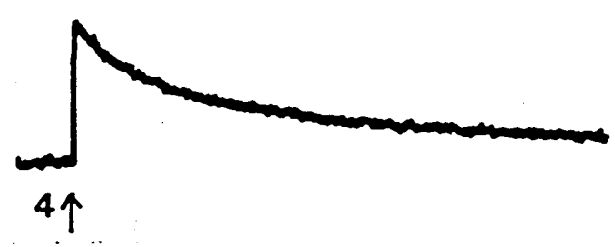

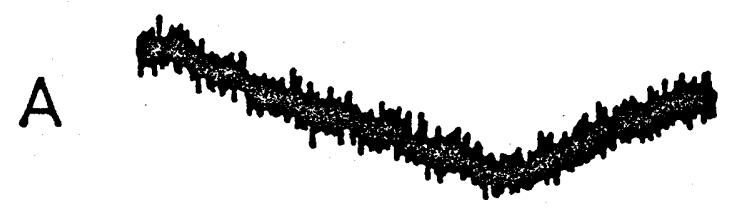

5

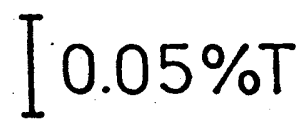

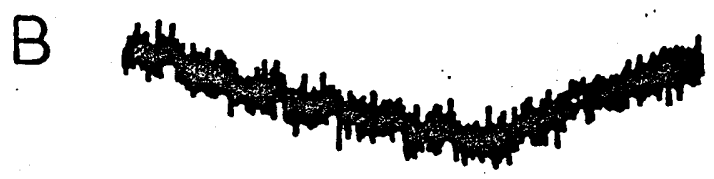

6

C

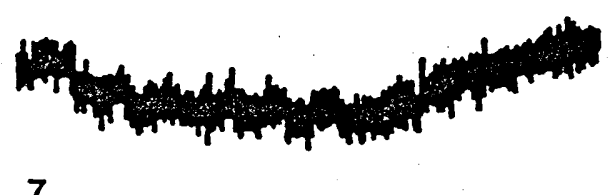

$.0 .2 \mathrm{~S}$

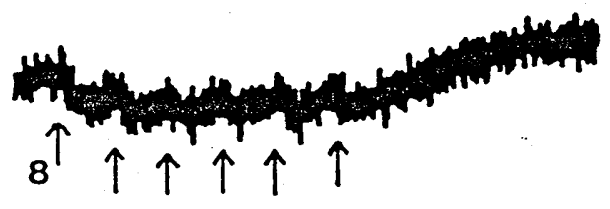

FIGURE 47 .

The effect of trypsin treatment in the light on the electrochromic bandshift and the oxonol VI absorption change. Thylakoids with a chlorophyll concentration of $25 \mathrm{Mgml}^{-1}$ were incubated with trypsin ( $1 \mu \mathrm{gmi}^{-1}$ ) in the dark in a medium containing $20 \mathrm{mM}$ Tricine, $10 \mathrm{mM} \mathrm{KCl}$ and $2 \mathrm{mM}$ $\mathrm{MgCl}_{2 ;}$. $\mathrm{pH} 7.4$. The reaction was stopped by adding trypsin inhibitor $\left(0.5 \mathrm{mgmi}^{-1}\right)$ followed by oxonol VI $(1.0 \mu M)$. The ellectrochromic bandshift signals $(1-4)$ were obtained by summing 64 single flash sweeps with a frequency of $1 \mathrm{~s}^{-1}$ whilst the oxonol VI signals $(5-8)$ were the summation of 128 sweeps fired at a frequency of 1 per $5 \mathrm{~s}$ - each sweep was composed of a train of 6 flashes spaced $90 \mathrm{~ms}$ apart. Trypsin incubation times were: A) Zero; B) $1 \mathrm{~min}$; $\ddot{k}$ C) $2 \mathrm{~min}$; D) $3 \mathrm{~min}$. 


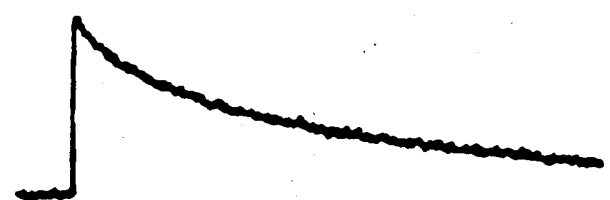

1

[0.07\% T

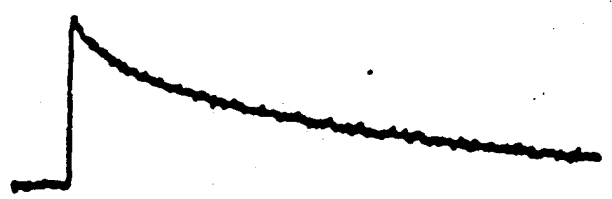

2

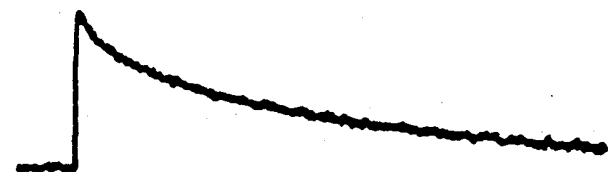

3.
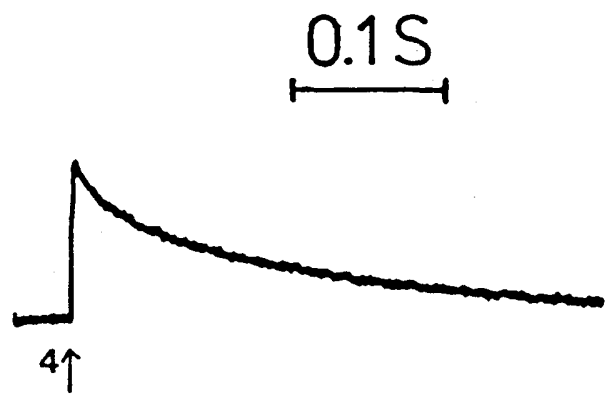

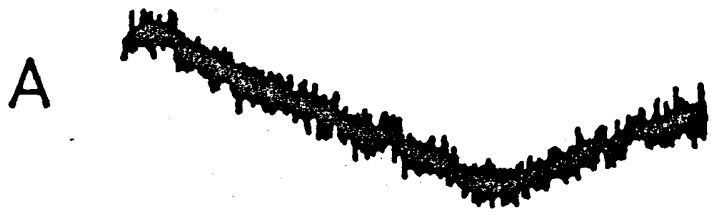

5

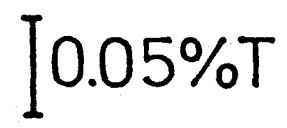

Q

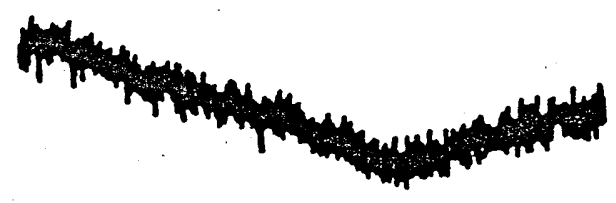

6

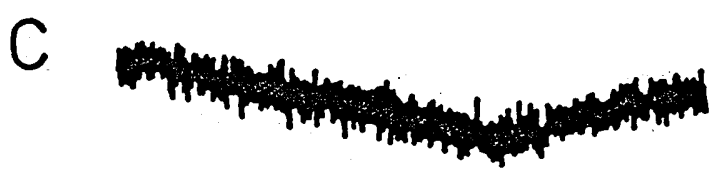

7.

FIGURE 48 .

The effect of chymotrypsin in the dark on the electrochromic bandshift and the oxonol VI absorption change. Thylakoids with a chlorophyll concentration of $25 \mu \mathrm{gml}^{-1}$ were incubated with chymotrypsin $\left(15 \mu \mathrm{gm} \mathrm{1}^{-1}\right)$ in the dark in a medium containing $20 \mathrm{mM}$ Tricine, $10 \mathrm{mM} \mathrm{KCl}$ and $2 \mathrm{mM}$ $\mathrm{MgCl}_{2} \mathrm{pH} \mathrm{7:4.} \mathrm{The} \mathrm{reaction} \mathrm{was} \mathrm{stopped} \mathrm{by}$ adding trypsin inhibitor $\left(0.5 \mathrm{mgml}^{-1}\right)$ followed by oxonol VI $(1.0 \mu \mathrm{M})$. The electrochromic bandshift signals $(1-4)$ were obtained by summing 64 single flash sweeps with a frequency of $1 \mathrm{~s}^{-1}$ whilst the oxonol VI signals $(5-8)$ were the summation of 128 sweeps fired at a frequency of 1 per 5 s - each sweep was composed of a train of 6 flashes spaced $90 \mathrm{~ms}$ apart. Chymotrypsin incubation times were: A) Zero; B) $1 \mathrm{~min}$; C) $2 \mathrm{~min}$; D) $3 \mathrm{~min}$. 


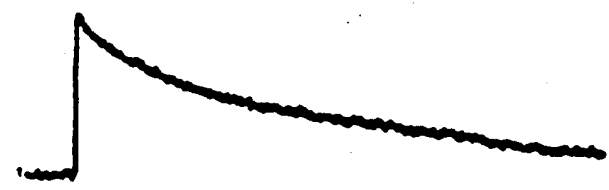

1

$[0.07 \% \mathrm{~T}$
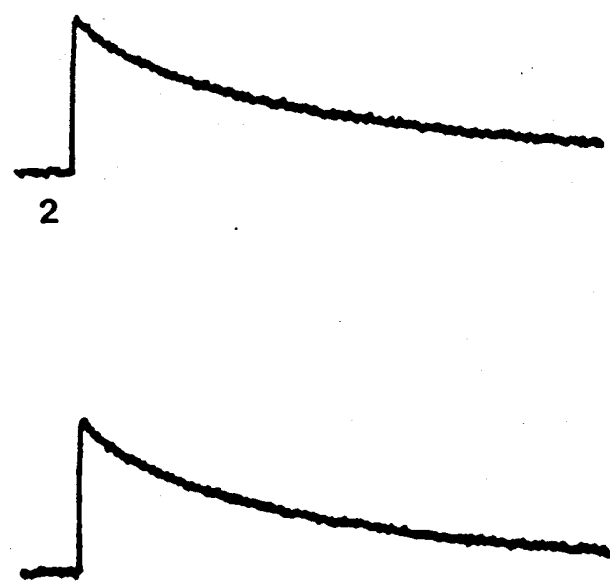

3
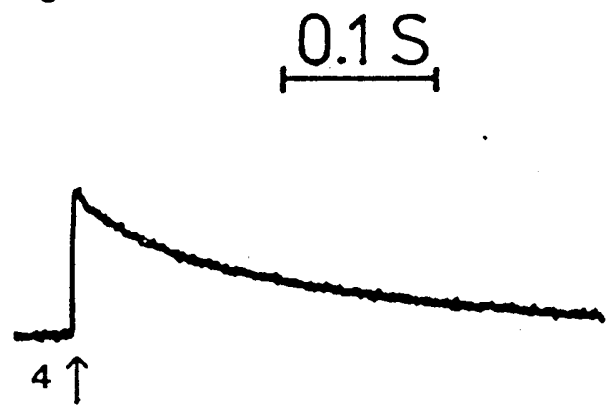

A

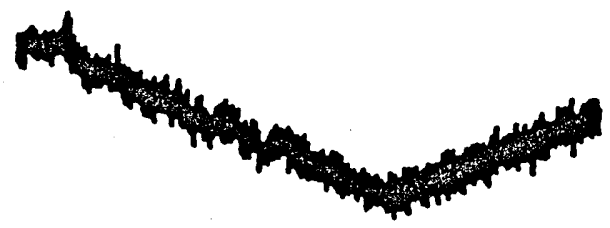

5

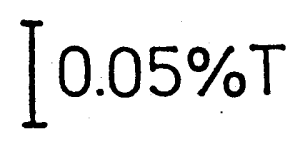

B

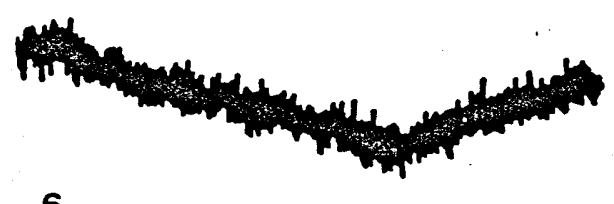

6

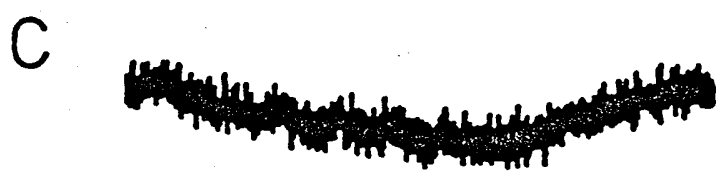

7

$D$

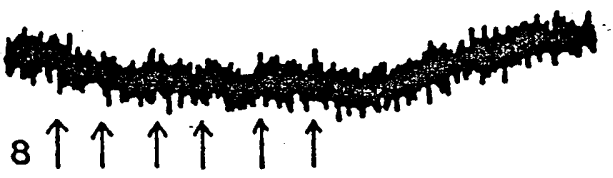

FIGURE 49 .

The effect of chymotrypsin in the light. on the electrochromic bandshift and the oxonol VI absorption change. Thylakoids with a chlorophyll concentration of $25 \mu^{-1}$ were incubated with chymotrypsin $\left(15 \mu g m 1^{-1}\right)$ in the dark in a medium containing $20 \mathrm{mM} \mathrm{Tricine,} 10 \mathrm{mM} \mathrm{KCl}$. and $2 \mathrm{mM}$ $\mathrm{MgCl}_{2} \mathrm{pH} 7.4$. The reaction was stopped by adding trypsin inhibitor $\left(0.5 \mathrm{mgml}^{-1}\right)$ followed by oxonol VI $(1.0 \mu \mathrm{M})$. The electrochromic bandshift signals $(1-4)$ were obtained by summing 64 single flash sweeps with a frequency of $1 \mathrm{~s}^{-1}$ whilst the oxonol VI signals $(5-8)$ were the summation of 128 sweeps fired at a frequency of 1 per $5 \mathrm{~s}$ - each sweep was composed of a train of 6 flashes spaced $90 \mathrm{~ms}$ apart. Chymotrypsin incubation times were: A) Zero; B) $1 \mathrm{~min}$; C) $2 \mathrm{~min}$; D) $3 \mathrm{~min}$. 

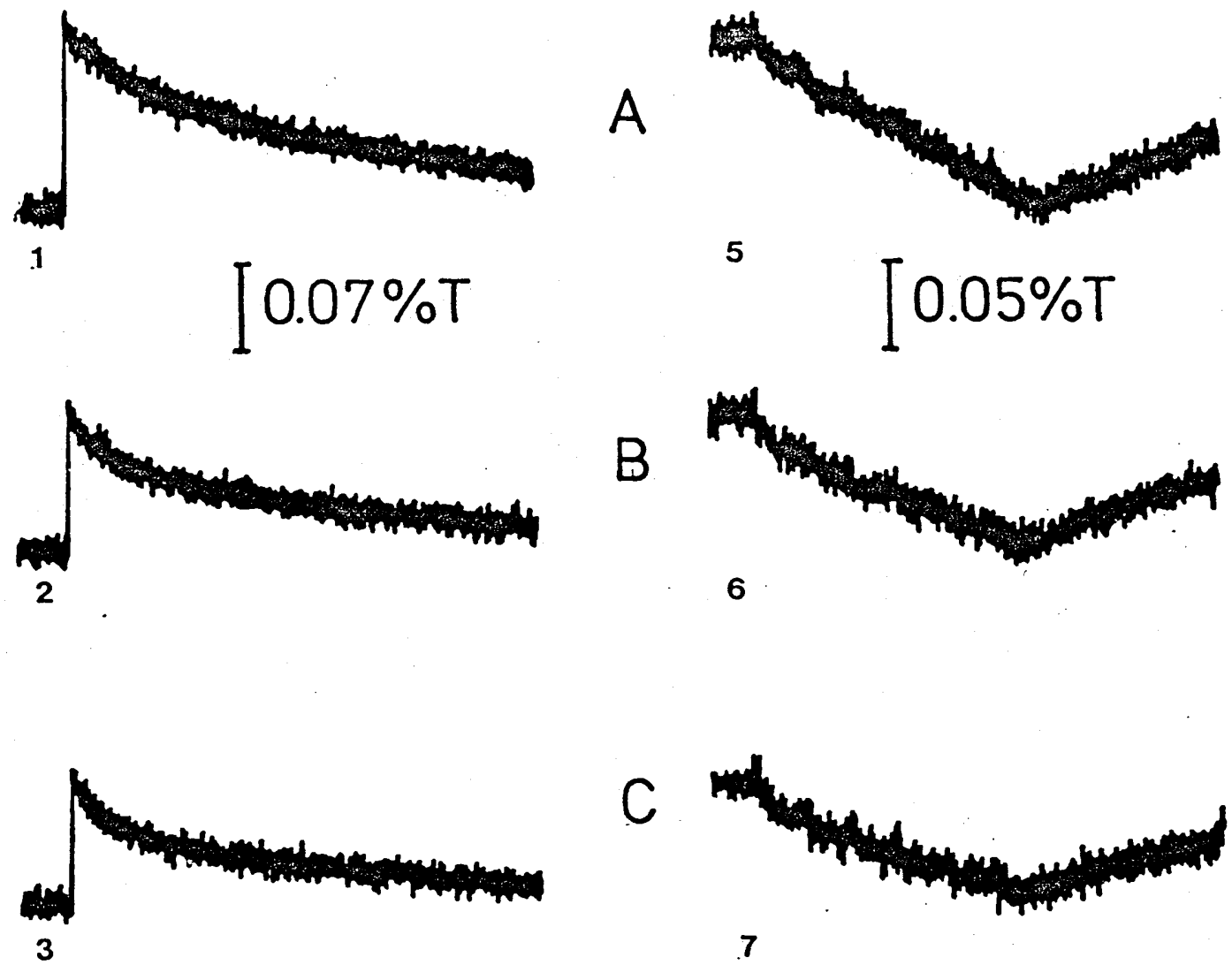

7
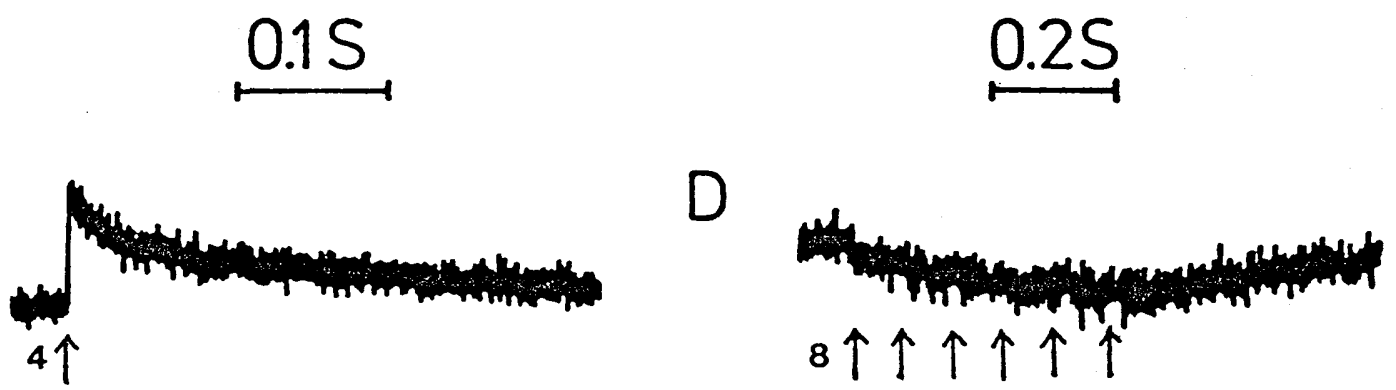

FIGURE 50 .

The effect of DCMU on the electrochromic bandshift and the oxonol VI absorption change. Thylakoids with a chlorophyll concentration of $25 \mathrm{Mgmi}^{-1}$ were resuspended in a medium containing $20 \mathrm{mM} \mathrm{Tricine,} 10 \mathrm{mM} \mathrm{KCl}, 2 \mathrm{mM}$ $\mathrm{MgCl}_{2}, \mathrm{pH} 7.4$ and oxonol VI $(1.0 \mu \mathrm{M})$. The electrochromic bandshift signals $(1-4)$ were obtained by summing 64. single flash sweeps with a frequency of $1 \mathrm{~s}^{-1}$. Whilst the oxonol VI signals $(5-8)$ were the summation of 128 sweeps fired at a frequency of 1 per $5 \mathrm{~s}$ each sweep was composed of a train of 6 flashes spaced $90 \mathrm{~ms}$ apart. The concentration of DCMU was: A) Zero; B) $5.0 \times 10^{-8} \mathrm{M}$; C) $7.5 \times 10^{-8} \mathrm{M}$;

D) $2.5 \times 10^{-7} \mathrm{M}$. 


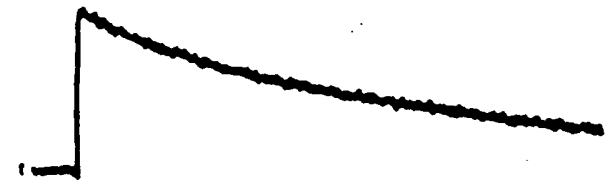

1
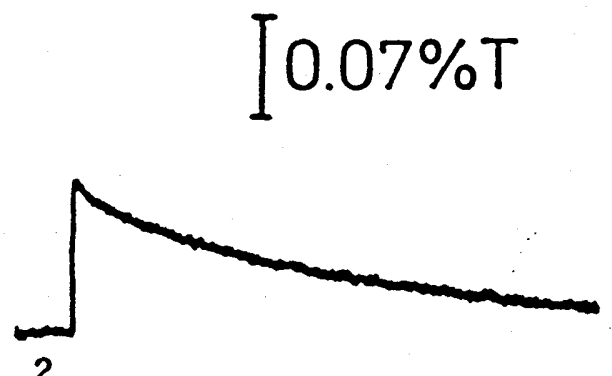

2

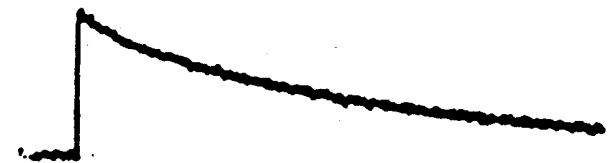

3
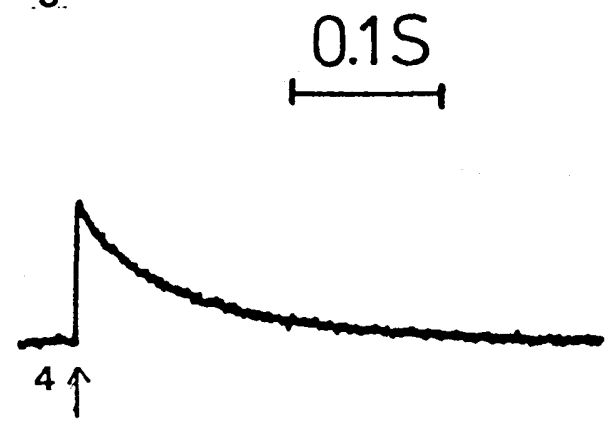

A

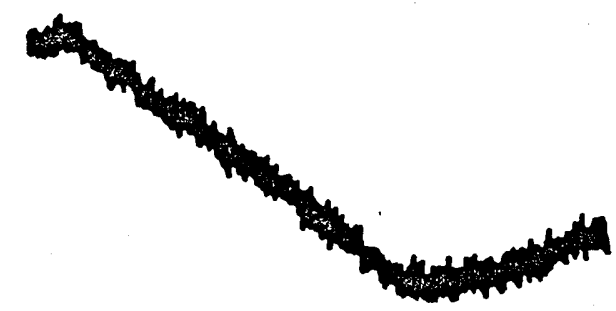

5

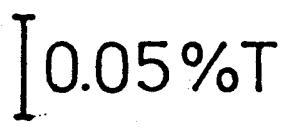

Q

6

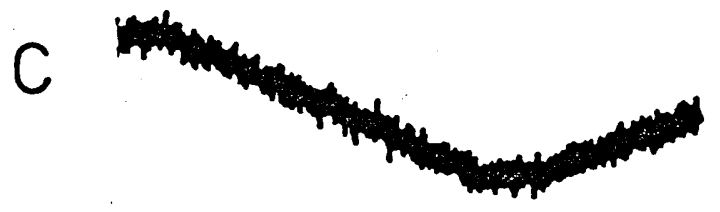

7

D

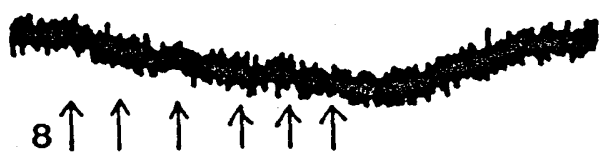

FIGURE 51 .

The effect of gramicidin on the electrochromic bandshift and the oxonol VI absorption change. Thylakoids with a chlorophyll concentration of

$25 \mathrm{MgmI}^{-1}$ were resuspended in a medium containing $20 \mathrm{mM} \mathrm{Tricine,} 10 \mathrm{mM} \mathrm{KCl}, 2 \mathrm{mM} \mathrm{MgCl} \mathrm{MH}_{2}, 4$ and oxonol VI (1.0 MM). The electrochromic bandshift signals $(1-4)$ were obtained by summing 64 single flash sweeps with a frequency of $1 s^{-1}$ whilst the oxonol VI signals $(5-8)$ were the summation of 128 sweeps fired at a frequency of 1 per $5 \mathrm{~s}-$ each sweep was composed of a train of 6 flashes spaced $9 \mathrm{cms}$ apart. The concentration of gramicidin was: A) $(\mathrm{ero} ; \mathrm{B}) 5.0 \times 10^{-12} \mathrm{M}$; C) $2.5 \times 10^{-1} 1 \mathrm{M}$ and D) $5 \times 10^{-11} \mathrm{M}$. 
is also believed to indicate this . it was concluded that a certain 'critical' potential is required before an oxonol VI absorbance change is seen.

Gramicidin is an ionophorous antibiotic that can increase the half-time decay rate of the membiane potential; the effect of this ionophore on the electrochromic bandshift and on the oxonol VI absorption change are shown in Figure 47 . This result shows that as the half-time of the decay rate of the bandshift decreases the amplitude of the oxonol VI change is reduced. It would appear that once a certain rate of decay of the membrane potential is reached oxonol VI is unable to respond because it needs a sufficiently slow decay in order to respond to it.

\subsection{DISC}

In Chapter 4 (Figs. 25-29) it was shown that both trypsin and chymotrypsin decreased the extent and the half-time of decay of the electrochromic bandshift. The aim of this investigation was to try to distinguish between the hypotheses (see 5.1) of why the extent of the bandshift was reduced by these enzymes. In this section it has been shown that the oxonol VI signal was almost completely inhibited by, trypsin or chymotrypsin, treatment under conditions which reduced the half-time of decay of the electrochromic bandshift but only marginally reduced the extent. From these results it was difficult to tell if the loss of oxonol VI signal was due to a real decrease in the magnitude of the field or whether it was due to the inability of oxonol VI to respond to the rapidly-decaying field. To differentiate between these two possibilities the effects of DCMU and gramicidin on the electrochromic 
bandshift and the oxonol VI absorption change were investigated. DCMU and gramicidin had distinctly different effects, as expected: DCMU reduced the amplitude by $50 \%$ when used at a concentration of $2.5 \times 10^{-7} \mathrm{M}$; gramicidin on the other hand did not affect the amplitude but markedly reduced the half-time decay. However, the oxonol VI abscrption change was completely inhibited by either of these compounds. This result suggested that oxonol VI could not distinguish between a reduction in the membrane potential or a decrease in the half-time of decay. The inability of oxonol VI to act differently in the presence of DCMU and gramicidin may be 1 inked to the slow response time of this probe (c.f. Admon et al, 1982). It was suggested previously that the movement of oxonol VI across the thylakoid membrane may be the limiting factor, particularly under conditions where the membrane potential is changing rapidly (Galmiche and Girault, 1981). The results presented here are in agreement with this proposal.

An alternative explanation for the oxonol VI response was suggested by Schuurmans et al (1981). They proposed that oxonol VI responds to the slow rise phase of the electrochromic bandshift which they associated with a slow electrogenic step involving proton displacement at the bulk-membrane interface. This slow phase was called reaction II by Schapendonk et al (1979)and it was proposed that this phase was not associated with the electrogenic steps at the reaction centres. Peters et al (1984) interpretated the results obtained from experiments using PSI-enriched membranes according to the reaction II theory (Schapendonk et. al,1979). They measured the electrochromic bandshift and the oxonolvI 
absorption change in PSI enriched fractions before and after the addition of ferredoxin. It was shown that in the presence of ferredoxin a slow rise phase of the bandshift and a previously absent oxonol VI signal became evident. Peters et al (1984) concluded from this that oxonol VI was responding to the slow rising phase only and was not an indicator of the membrane potential created at the photosystems. However, looking at the electrochromic bandshift signal produced by the PSI-particles, in the absence of ferredoxin, shows that the half-time of decay was fairly rapid ( $\sim 50 \mathrm{~ms})$. The data presented in this Chapter would predict that oxonol VI would be unable to respond to such a rapidly changing membrane potential. Therefore it seems reasonable to suggest that the oxonol VI response was more marked in samples displaying a slow rise phase in the bandshift signal due to the slower rate of decay in these samples and not because the slow phase is a different type of reaction. Therefore the results presented here give no support to the proposals of schuurmans et al (1981) and Peters et a ㅅ (1984).

In conclusion the oxonol VI probe turned out to be more sensitive to enzyme treatments than the electrochromic bandshift. Therefore it was not possible to use this probe to see if trypsin and chymotrypsin were digesting the bandshift sensing pigments. However, the results presented here have emphasised the idea that oxonol VI is only useful as an indicator of the membrane potential when it is changing slowly. 


\section{CHAPTER SIX - EFFECTS OF TRYPSIN_AND CHYMOTRYPSIN ON P P II-MEDIATED REACTIIONS.}


Effects of trypsin and chymotrypsin on PSIImediated reactions.

\subsection{INTRODUCTION.}

Trypsin and chymotrypsin were shown to cause a decrease in the extent of the electrochromic bandshift, with trypsin having a considerably more marked effect than chymotrypsin. As was discussed in the previous chapter this could be due to a number of factors including: 1) a loss of pigments which sense the electrochromic bandshift; 2) a loss of a portion of the light harvesting complex protein (LHCP) responsible for light energy capture and subsequent excitation energy transfer to the reaction centres; and ' 3 ) a reduction in the efficiency in one or both of the photosystems by a) a direct effect on the reaction centre proteins or b) a redistribution of energy between PSII and PSI which could result in an overall decrease in charge separation at the two photosystems. The investigation here is concerned with the effects of trypsin and chymotrypsin on the PSII- ard PSIinduced membrane potential.

Trypsin is known to digest a $32 \mathrm{kDa}$ protein associated with the acceptor side of PSII (Mullet and Arntzen, 1981 ) and the results in chapter 3 indicated that chymotrypsin may also digest this protein. However, al though both trypsin $\left(50 \mu \mathrm{gml} \mathrm{m}^{-1}, 5-10 \mathrm{~min}\right)$ and chymotrypsin $\left(100 \mu \mathrm{g} \mathrm{m} \mathrm{l}^{-1}, 5-10 \mathrm{~min}\right)$ treatments appear to affect the acceptor side of PSII, there is a significant difference between the effects of these enzymes on the extent of the bandshift. In Chapter 4 it was shown (Figs 25-28) that trypsin treatment 
(1.5 $\left.\mu \mathrm{g} \mathrm{ml} \mathrm{l}^{-1}, 1-5 \mathrm{~min}\right)$ reduced the amplitude of the bandshift by $70 \%$ whilst chymotrypsin treatment ( $5 \mu \mathrm{g} \mathrm{ml}, 1-5 \mathrm{~min}$ ) caused only a $40 \%$ reduction.

Trypsin is known to remove a portion of the LHCP which has been implicated in the regulation of energy transfer between the two photosystems (Burke et al, 1978 ; Steinback et al 1979). Bearing this in mind the effects of these enzymes were investigated.

\section{6 . 2 RES UULTS.}

6.2.1 The effects of trypsin and chymotrypsin on the electrochromic bandshift in thylakoids resuspended in low-or high-salt media.

Figures 52 and 53 show: the flash-induced electrochromic bandshift at $520 \mathrm{~nm}$ obtained when thylakoids were treated with trypsin and chymotrypsin in media containing either $10 \mathrm{mM} \mathrm{NaCl}$ but no divalent cations (low-salt) or $10 \mathrm{mM} \mathrm{NaCl}$ and $2 \mathrm{mM} \mathrm{Mg}^{2+}$ (high-salt). It can be seen (Figs 52 or 53 ) that resuspension of chloroplasts in a high-salt medium resulted in a greater amplitude of the electrochromic bandshift than in similar samples resuspended in low-salt medium. This could be due to a reduction in the PSII mediated membrane potential: 1) caused by conformational changes in PSII (Bose and Arntzen, 1978); or 2) by redistribution of excitation energy, away from PSII in favour of PSI, provided PSI was already maximally excited. Trypsin caused a reduction in the extent of the electrochromic bandshift in both high- and lowsalt samples (Fig 52). This, effect was more marked in samples treated with trypsin in high- salt than those in low- salt media: after $3 \mathrm{~min}$ incubation, 


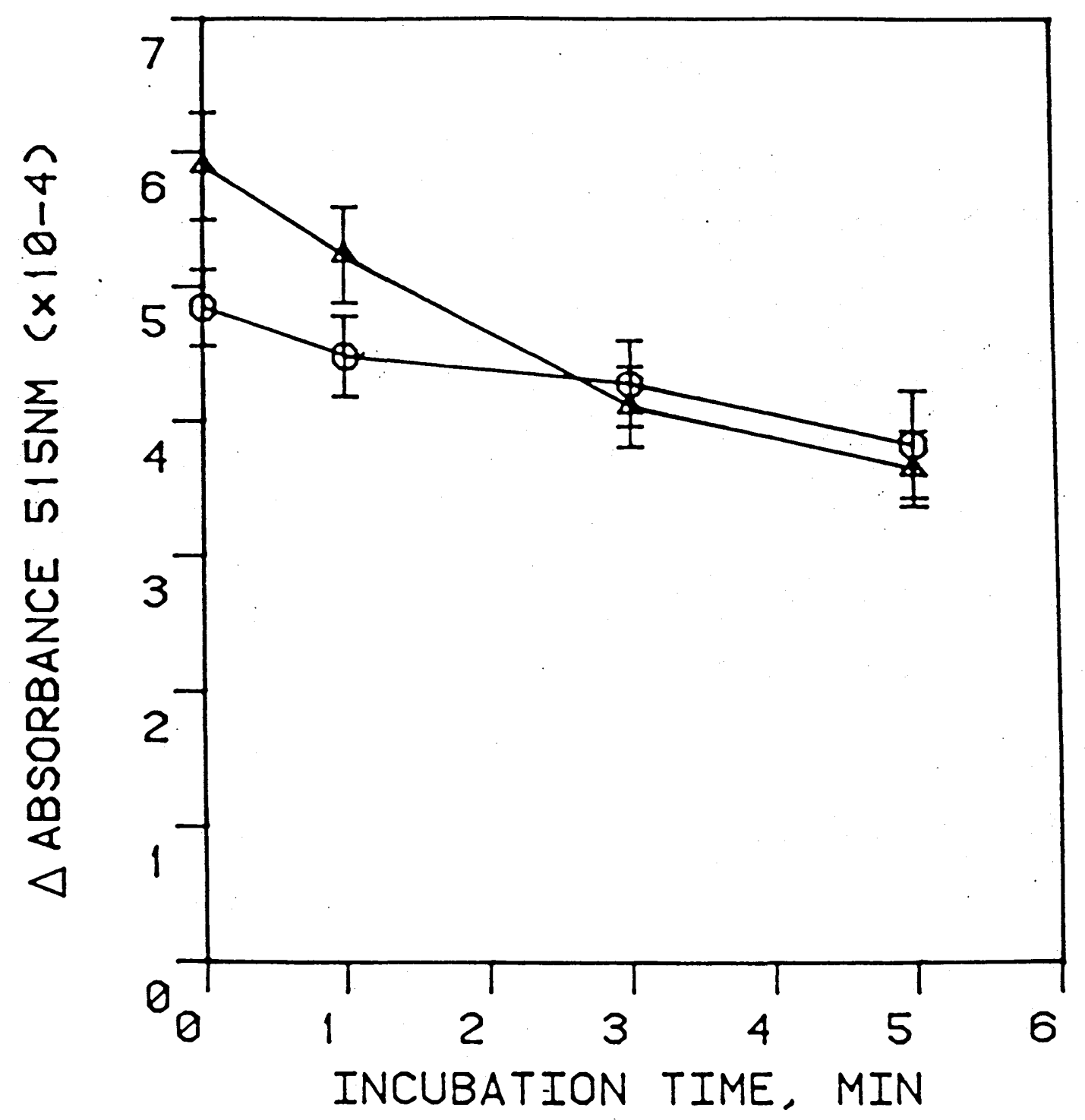

FIGURE 52 .

The effect of trypsin on the amplitude of the electrochromic bandshift. Thylakoids with a chlorophyll concentration of $25 \mu^{-1}$ were treated with trypsin ( $\left.1.5 \mu \mathrm{gmi}^{-1}\right)$ in a medium containing $20 \mathrm{mM}$ Tricine, $10 \mathrm{mM} \mathrm{KCl}$ containing either no divalent cations (0) or $2 \mathrm{mM} \mathrm{MgCl} 2$ $(\Delta) \mathrm{pH} 7,4$. The reaction was stopped by adding $0.5 \mathrm{mg} \mathrm{ml}^{-1}$ of trypsin inhibitor. Potassium ferricyanide $(7.5 \mathrm{mM})$ was present as electron acceptor. 


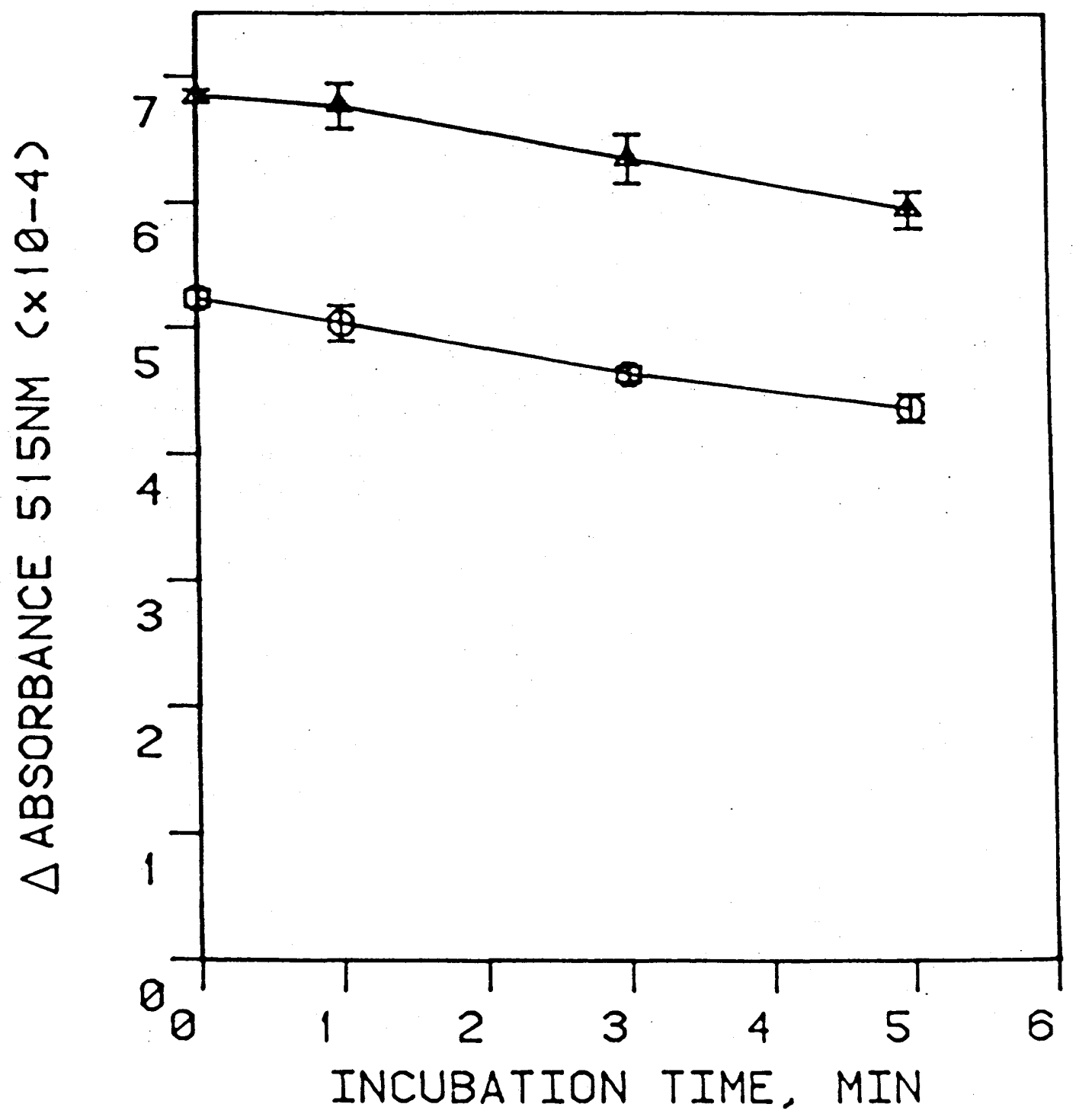

*FIGURE 53.

The effect of chymotrypsin on the amplitude of the electrochromic bandshift. Thylakoids with a chlorophyll concentration of $255^{4 g m l^{-1}}$ were treated with chymotrynsin ( $\left.5 \mu \mathrm{gml}^{-1}\right)$ in a nedium containing $20 \mathrm{mM}$ Tricine, $10 \mathrm{mM} \mathrm{KCl}$ containing either no divalent cations (0) or $? \mathrm{mM} \mathrm{MgCl} 2(\Delta) \mathrm{pH} 7.4$. The reaction was stopped by adding trypsin inhibitor $\left(0.5 \mathrm{mgml}^{-1}\right)$. Potassium ferricyanide $(1.5 \mathrm{mM})$ was added as electron acceptor. 
the extent of the bandshift in the high- salt samples was reduced to the level of similarly treated low-salt thylakoids. In the case of chymotrypsin treatment (Fig 53) there was no difference observed in the effects of this enzyme in either low- or high- salt media. The data show (Fig. 53) that in both high- and lowsalt samples that chymotrypsin reduced the extent to a similar degree. This differential effect of these enzymes may be due to the ability of trypsin to remove a portion of LHCP, associated with PSII, which is involved in the maintenance of granal stacks and in the control of energy redistribution between the two photosystems.

6.2.2 The effects of trypsin and chymotrypsin on the

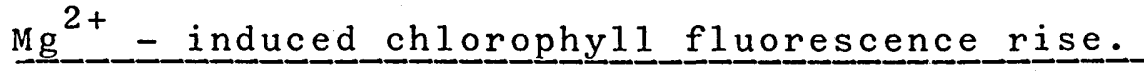

Addition of $\mathrm{Mg}^{2+}$ to thylakoids resuspended in a lowsalt medium resulted in the well known increase in fluorescence, which has been correlated with the restacking of grana (Akoyunoglou: and Akoyunoglou, 1977). The effects of trypsin and chymotrypsin on this fluorescence rise are shown in Figure 54. It can be seen that chymotrypsin had almost no effect on this phenomenon but trypsin almost completely inhibited the rise after $5 \mathrm{~min}$ incubation. This result indicated that trypsin may inhibit energy redistribution changes and granal stacking which are known to be induced by $\mathrm{Mg}^{2+}$. Electron micrographs (Fig. 55) show the effect of trypsin and chymotrypsin on the morphology of thylakoid membranes. It can be seen that resuspension of isolated thylakoids in high-salt (Fig. 55D) allowed them to retain the granal stacks found in viveo. 


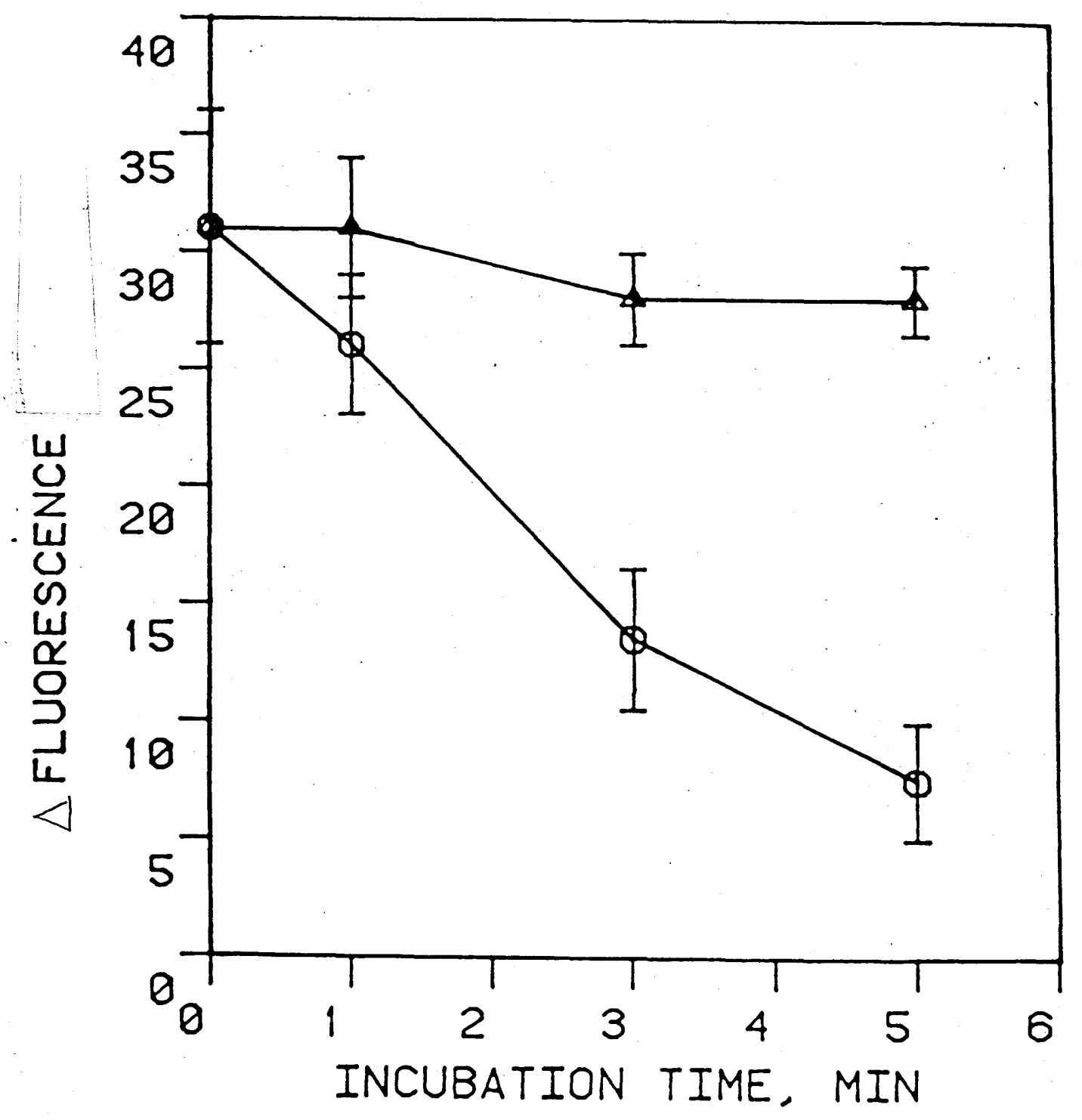

*FIGURE 54.

The, effect of trypsin and chymotrypsin on the $\mathrm{Mg}^{2+}$ - induced chlorophyll fluorescence rise. Thylakoids with a chlorophyll concentration of $5 \mu \mathrm{gml}^{-1}$ were treated with trypsin $\left(0.3 \mu \mathrm{gml} \mathrm{I}^{-1}\right)$ (o) or chymotrypsin $\left(1.25 \mu \mathrm{gm} 1^{-1}\right)(\Delta)$ in a medium containing $20 \mathrm{mM}$ Tricine and 10 in $\mathrm{KCl}$ $\mathrm{pH}$ 7.4. The reaction was stopped by adding trypsin inhibitor $\left(0.15 \mathrm{mgml}^{-1}\right)$. DCMU (10 $\left.\mu \mathrm{M}\right)$ was added to the sample and the level of fluorescence recorded. then $2 \mathrm{mM} \mathrm{MgCl}_{2}$ was added. After $5 \mathrm{~min}$ a second fluorescence measurement was made. 


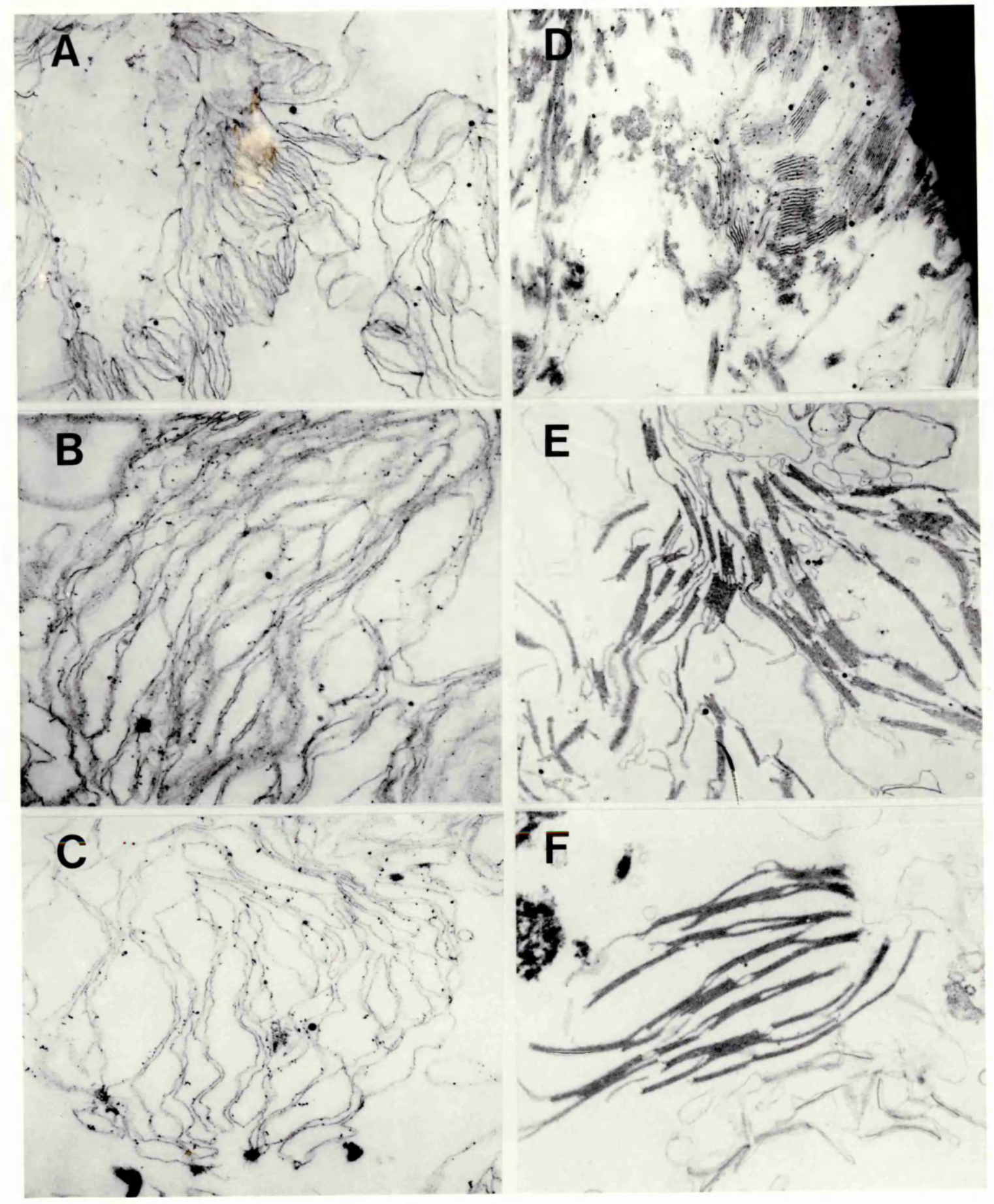

FIGURE 55.

The effects of trypsin and chymotrypsin on the morphology of the thylakoid membranes. Thylakoids with a chlorophyli concentration of $25 \mu \mathrm{g} \mathrm{m} \mathrm{I}^{-1}$ were incubated with trypsin $\left(1.5 \mu \mathrm{g} \mathrm{ml^{-1 }}\right)$ or chymotrypsin ( $5 \mu \mathrm{g} \mathrm{ml}^{-1}$ ) in a medium containing $20 \mathrm{mM}$ Tricine, $10 \mathrm{mM} \mathrm{KCl}$ and $2 \mathrm{mM}$ $\mathrm{MgCl}_{2} \mathrm{pH} 7.4$. The reaction was stopped by adding trypsin inhibitor $\left(0.5 \mathrm{mg} \mathrm{m} \mathrm{m}^{-1}\right)$. A) Control (low salt); B) trypsin $1 \mathrm{~min}$; C) trypsin 5 min; D) Control (high salt); E) chymotrypsin 1 min; F) chymotrypsin 5 min. 
on the other hand resuspension in low- salt caused granal destacking (Fig 55A). Figure 55 shows that trypsin induced unstacking of grana while chymotrypsin had no apparent effect on the grana. These results suggest that trypsin is removing the portion of the LHCP which has been implicated in energy redistribution and in the maintenance of granal stacks, whilst chymotrypsin does not appear to affect this polypeptide.

6.2.3 The effects of trypsin and chymotrypsin on the electrochromic bandshift induced by PSI al one.

The data from this investigation are shown in Figure 56: treatment of thylakoids with trypsin resulted in a small but significant increase in the electrochromic bandshift associated with PSI only, whereas chymotrypsin caused a small decrease in this signal. The results shown here are from experiments using TMPD and ascorbate as the electron donor pair but experiments using DCPIP and ascorbate gave similar results (data not shown). Measurements of the electrochromic bandshift from high- and low- salt controls showed that the extent of the PSI-induced bandshift signal was greater in high-salt than in similar low- salt samples. These results suggest that trypsin inhibits the PSII- mediated membrane potential and simultaneously increases the PSI response. 


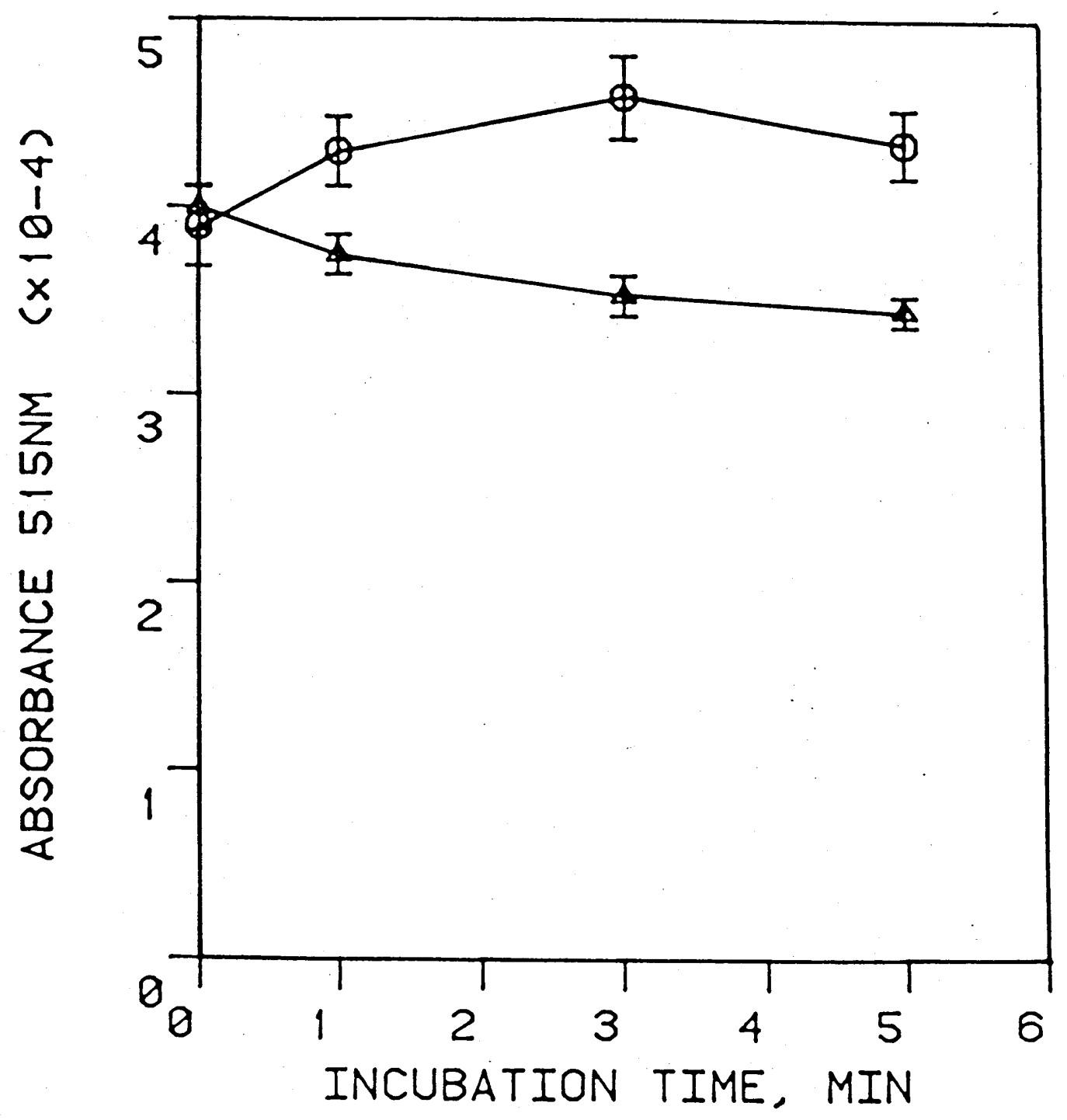

FIGURE 56.

The effects of trypsin and chymotrypsin on the amplitude of the electrochromic bandshift induced by PSI alone. Thylakoids with a chlorophyll concentration of $25 \mu \mathrm{g} \mathrm{ml}^{-1}$ were incubated with trypsin $(1.5 \mu \mathrm{g} \mathrm{m1-1})(0)$ or chymotrypsin $\left(5 \mathrm{Hg} \mathrm{m}^{-1}\right)(\Delta)$ in a medium containing $20 \mathrm{mM}$ Tricine, $10 \mathrm{mM} \mathrm{KCl}$ and $2 \mathrm{mM} \mathrm{MgCl} 2, \mathrm{pH} \mathrm{7.4.} \mathrm{The}$ reaction was stopped by adding trypsin inhibitor $\left(0.5 \mathrm{mg} \mathrm{ml^{-1 }}\right)$. PSII was inhibited by DCMU $(10 \mu \mathrm{M})$ and hydroxylamine $(100 \mu \mathrm{M})$. TMPD $(0.2$ $\mathrm{mM}$ ) and ascorbate ( $3 \mathrm{mM}$ ) were added as donors to PSI. (See Materials and Methods for detalls). 
6.2.4 The relative flash yield of oxygen as a function of 1 ight intersity.

Figure 57 shows that incubating thylakoids in the presence of trypsin ( 5 nin) altered the shape of the light-saturation curve of flash-induced oxygen evolution when compared with that of the untreated samples. Thylakoids treated with trypsin required a higher light intensity in order to yield the same relative amount of oxygen as untreated membranes. on the other hand chymotrypsin ( $5 \mathrm{~min}^{\prime}$ ) (Fig 58) gave a result which was essentially the same as the control. This result again suggests that trypsin is affecting the LHCP associated with 1 ight harvesting to PSII whilst chymotrypsin does not.

6.2.5 Induction of chlorophyll fluorescence in DCMU - blocked thylakoid membranes.

The level of variable chlorophyll fluorescence $\left(\mathrm{F}_{\mathrm{v}}\right)$ in DCMU-blocked thylakoids was obtained by making measurements of rapid fluorescence induction curves from which the $F_{0}$ level (minimum fluorescence) and $F_{m}$ level (maximum fluorescence) were obtained $\left(F_{\mathrm{v}}=\mathrm{F}_{\mathrm{m}}-\mathrm{F}_{\mathrm{o}}\right)$. The data presented in Figures 59 and 60 is given as values of $\mathrm{F}_{\mathrm{v}} / \mathrm{F}_{\mathrm{m}}$ which is a measure of the photochemical efficiency of PSII: the closer $\mathrm{F}_{\mathrm{V}} / \mathrm{F}_{\mathrm{m}}$ is to 1 the greater the efficiency;conversely the lower the value of $\mathrm{F}_{\mathrm{v}} / \mathrm{F}_{\mathrm{m}}$ the lower the efficiency (see 1.9.2 for more details on theory). The DCMU binding is not affected by the enzymes because they are being used at very low concentrations. Figure 59 shows the effects of trypsin on $\mathrm{F}_{\mathrm{v}} / \mathrm{F}_{\mathrm{m}}$ in thylakoids suspended in low- and high-salt media. It can be 


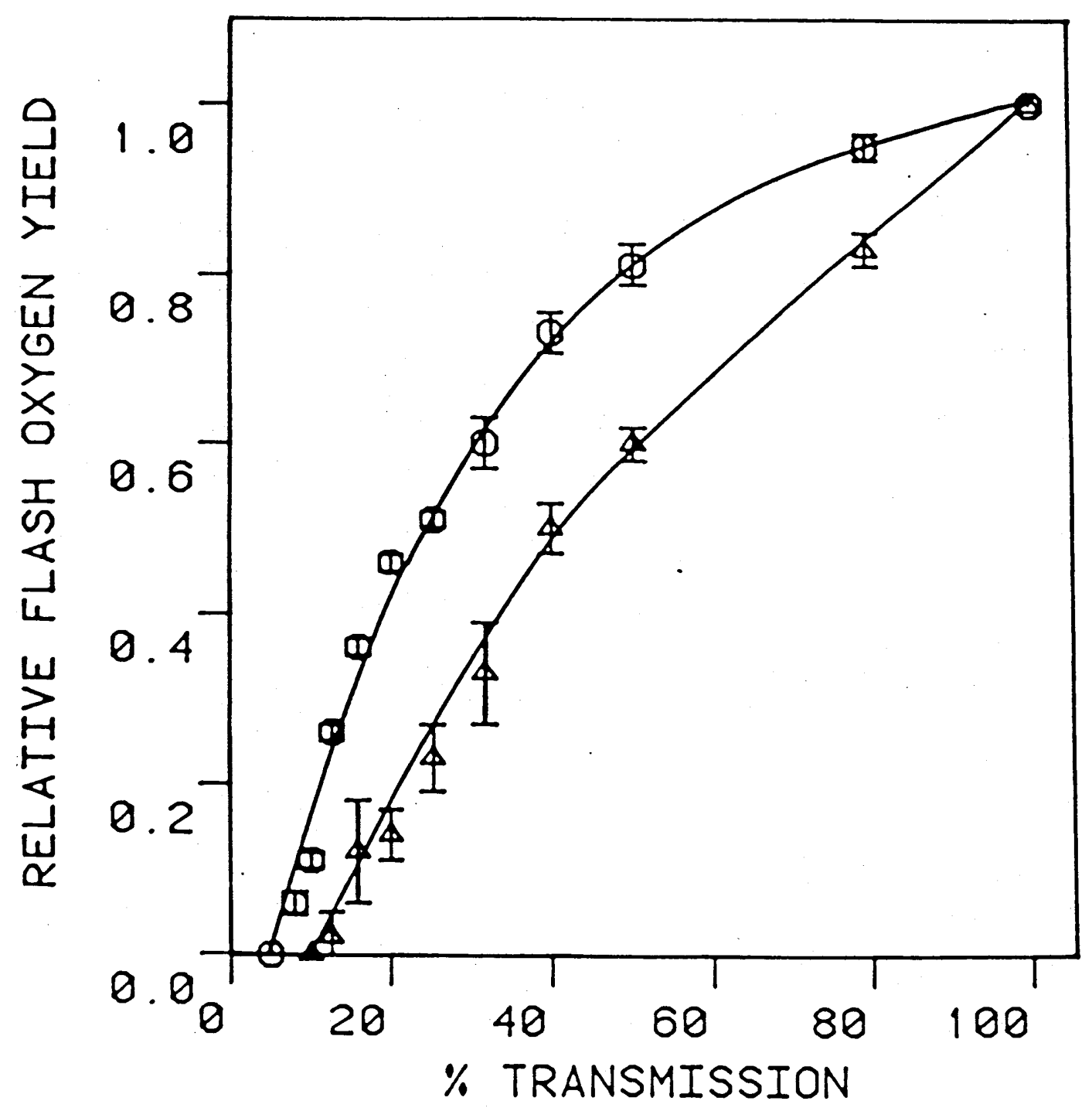

FIGURE 57 .

The relative flash oxygen yield as a function of flash intensity after thylakolds were treated with trypsin. Thylakoids with a chlorophyll concentration of $100 \mu \mathrm{ml}^{-1}$ were incubated with trypsin (6 $\mathrm{\mu g} \mathrm{ml}^{-1}$ ) in a medium containing $20 \mathrm{mM}$ Tricine, $10 \mathrm{mM} \mathrm{KCl}$ and $2 \mathrm{mM} \mathrm{MgCl} 2, \mathrm{pH} 7.4$. The reaction was stopped by adding trypsin inhibitor $\left(0.5 \mathrm{mg} \mathrm{ml^{-1 }}\right)$. The assay medium contained $20 \mathrm{mM}$ Tricine, $100 \mathrm{mM} \mathrm{KCl}, 2 \mathrm{mM} \mathrm{MgCl} 2$, $100 \mu \mathrm{M}$ NADP and $2 \mu \mathrm{M}$ Ferredoxin. Control, no treatment $(0)$, Trypsin-treated $(\Delta)$. 


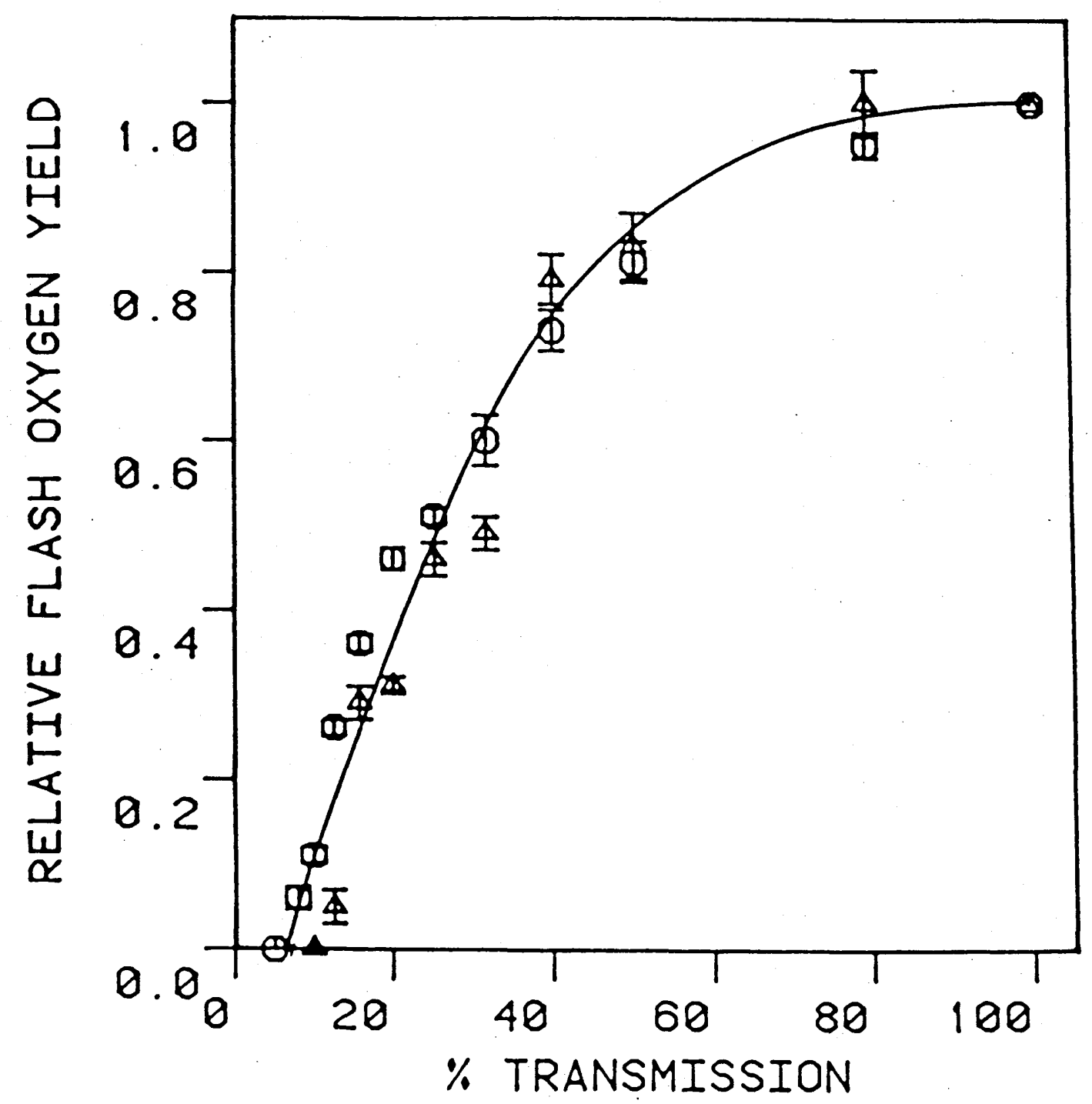

FIGURE 58 .

The relative flash oxygen yleld as a function of flash yield intensity after thylakoids were treated with chymotrypsin. Thylakoids with a chlorophyll concentration of $100 \mu \mathrm{g} \mathrm{ml^{-1 }}$ were incubated with chymotrypsin $\left(20 \mu \mathrm{g} \mathrm{ml^{-1 }}\right)$ in a medium containing $20 \mathrm{mM}$ Tricine, $10 \mathrm{mM}$ $\mathrm{KCl}$ and $2 \mathrm{mM} \mathrm{MgCl} 2, \mathrm{pH} 7.4$. The reaction was stopped by adding trypsin Inhibitor $\left(0.5 \mathrm{mg} \mathrm{ml^{-1 }}\right)$. The assay medium contained $20 \mathrm{mM}$ Tricine, 100 $\mathrm{mM} \mathrm{KCl}, 2 \mathrm{mM} \mathrm{MgCl}_{2}, 100 \mu \mathrm{M} \mathrm{NADP}$ and $2 \mu \mathrm{M}$ Ferredoxin. Control, no treatment (0); Chymotrypsin-treated $(\Delta)$. 


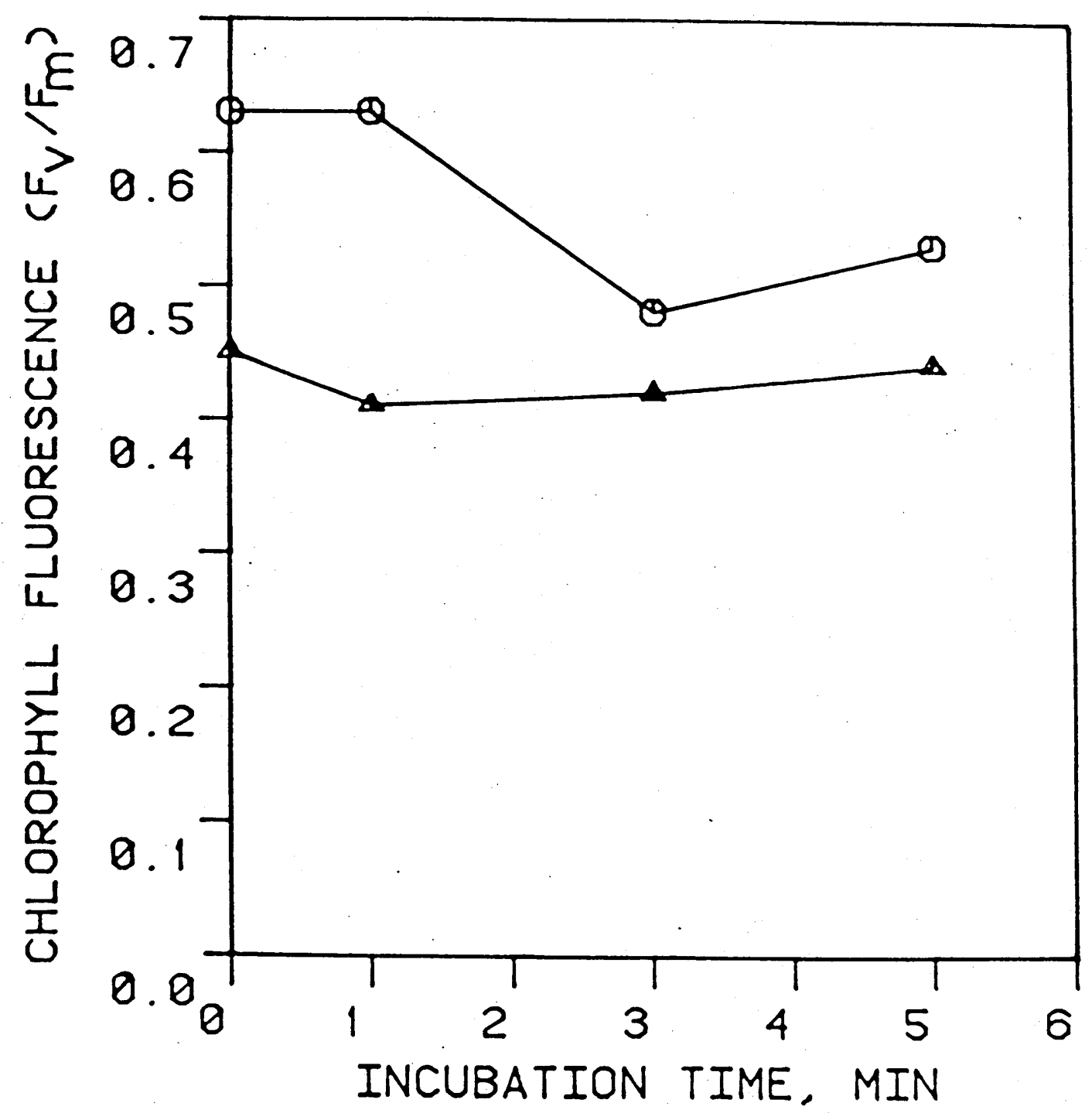

FIGURE 59 .

The effect of trypsin on chlorphyll fluorescence in thylakoids blocked with DCMU. Thylakoids with a chlorophyll concentration of $5 \mathrm{Mgml}^{-1}$ were treated with trypsin $\left(0.3 \mu \mathrm{gml}^{-1}\right)$ in a medium containing $20 \mathrm{mM}$ Tricine, $10 \mathrm{mM} \mathrm{KCl}$ with either no divalent cations $(\Delta)$ or $2 \mathrm{mM}$ $\mathrm{MgCl}_{2}(0)$. The reaction was stopped by adding trypsin inhibitor $\left(0.15 \mathrm{mgml}^{-1}\right)$ then DCMU (10 4 H) was added. 


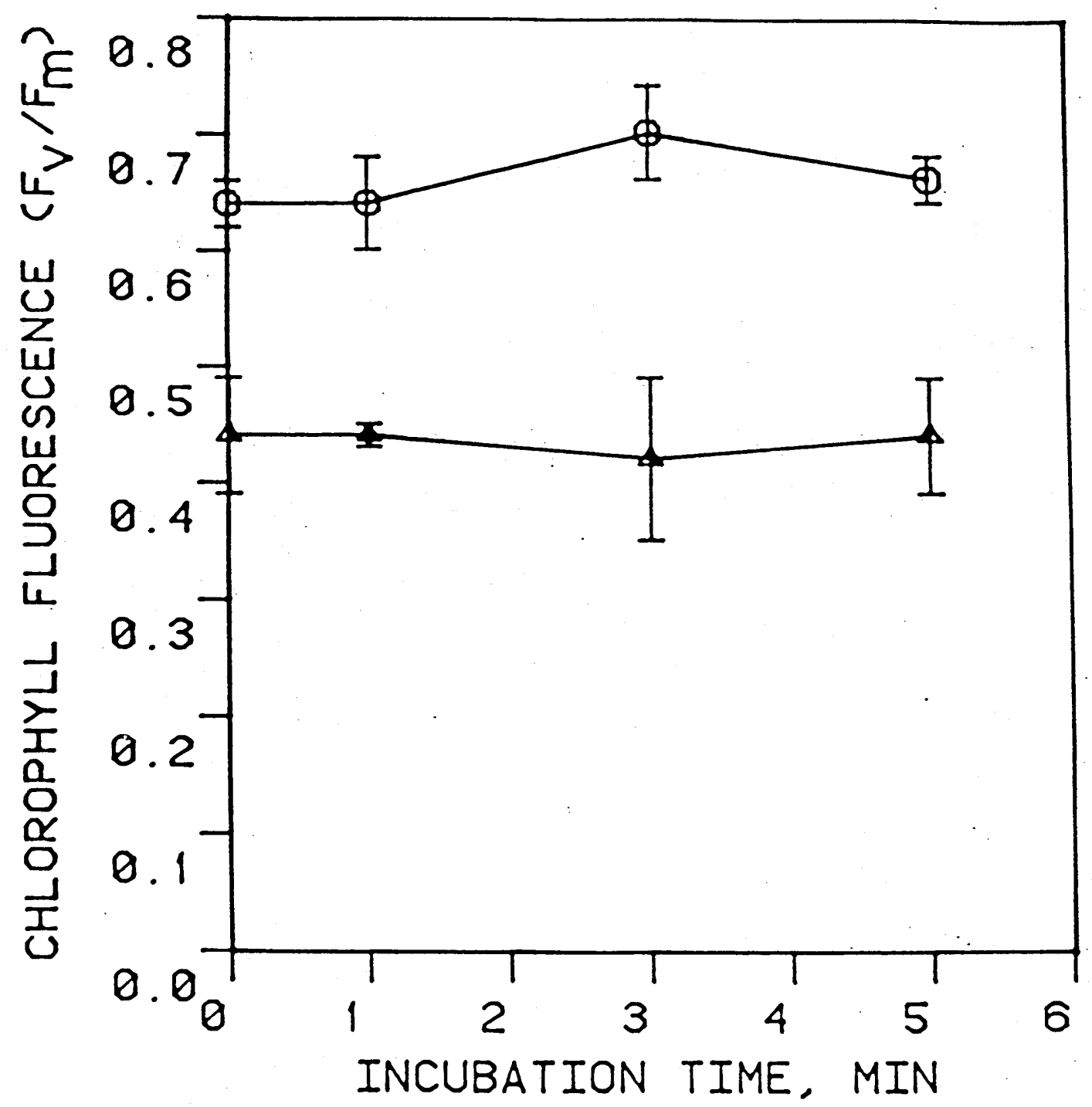

FIGURE 60 .

The effect of chymotrypsin on chlorophyll

fluorescence in thylakoids blocked with DCMU. Thylakoids with a chlorophyll concentration of $5 \mu \mathrm{gm} 1^{-1}$ were treated with chymotrypsin (1.25 $\mu \mathrm{gmi}^{-1}$ ) in a medium containing 20 mil Tricine, $10 \mathrm{mM} \mathrm{KCl}$ with either no divalent cations $(\Delta)$ or $2 \mathrm{mM} \mathrm{MgCl} 2(0)$. The reaction was. stopped by adding trypsin inhibitor $\left(0.15 \mathrm{mgml}^{-1}\right)$ then DCMU $(10 \mu M)$ was added. 
seen that $\mathrm{F}_{\mathrm{v}} / \mathrm{F}_{\mathrm{m}}$ was lower in control samples resuspended in low- salt than in similar high-salt samples. This indicates, as expected,that PSIImediated photochemistry is reduced in thylakoids resuspended in low-salt media. It can be seen that trypsin reduced $\mathrm{F}_{\mathrm{V}} / \mathrm{F}_{\mathrm{m}}$ from 0.62 to $\sim 0.5$ in thylakoids resuspend in high-salt. But in similarly treated thylakoids resuspended in low-salt, trypsin did not appear to affect $\mathrm{F}_{\mathrm{V}} / \mathrm{F}_{\mathrm{m}}$. The effect of chymotrypsin on thylakoids resuspended in low- and high- salt media is shown in Figure 60. It can be seen that, unlike trypsin, chymotrypsin had no effect on $\mathrm{F}_{\mathrm{v}} / \mathrm{F}_{\mathrm{m}}$ in either low- or high-salt media.

6.2.6 The effects of low concentrations of trypsin and chymotrypsin on the rate of 1 ight-induced oxygen evolution in uncoupled thylakoid membranes.

The rates of oxygen evolution were measured in thylakoids treated with trypsin. These membranes were uncoupled using $\mathrm{NH}_{4} \mathrm{Cl}$, and $\mathrm{p}$-benzoquin one or potassium ferricyanide were used as electron acceptors. Figure 61 shows that trypsin inhibited oxygen evolution in both cases; oxygen evolution using p-benzoquinone as acceptor wàs inhibited by $45 \%$ whilst ferricyanide mediated oxygen evolution was reduced by $30 \%$. Similar results were obtained for chymotrypsin (Fig 62) where it can be seen that the rate of oxygen evolution in the presence of ferricyanide or p-benzoquinone is reduced by $20 \%$ and $40 \%$ respectively. 


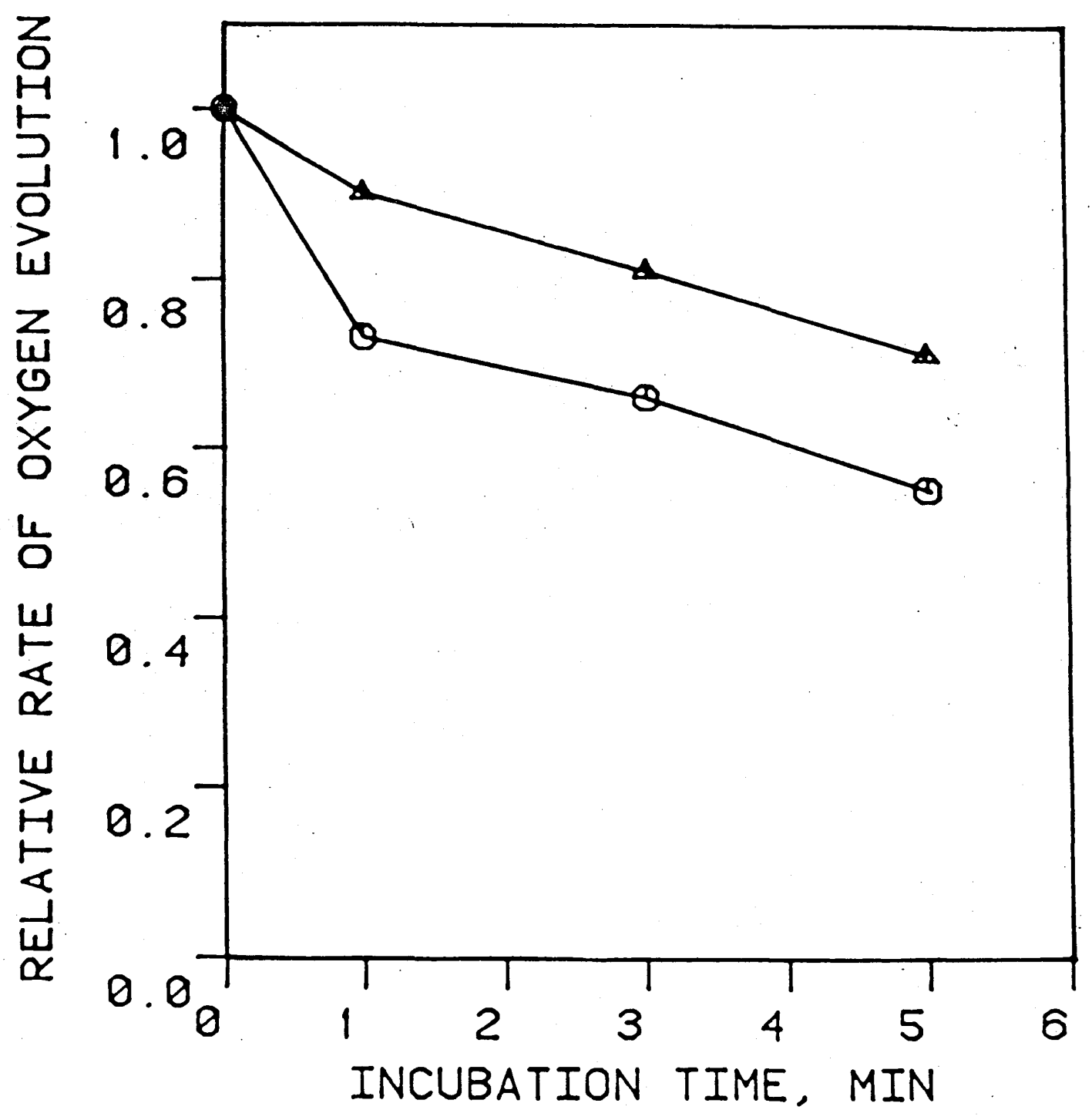

FIGURE 61.

The effect of low concentrations of trypsin on the rates of oxygen evolution in uncoupled thylakolds. Thylakolds with a chlorophyll concentration of $50 \mu \mathrm{g} \mathrm{ml^{-1 }}$ were treated with trypsin $\left(3 \mu \mathrm{g} \mathrm{ml}{ }^{-1}\right)$ in a medium containing $20 \mathrm{mM}$ Tricine, $10 \mathrm{mM} \mathrm{KCl}$ and $2 \mathrm{mM} \mathrm{MgCl} 2, \mathrm{pH} \mathrm{7.4.} \mathrm{The}$

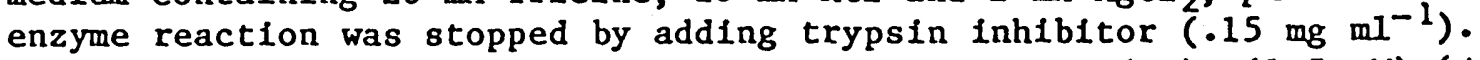
Measurements of oxygen evolution were made using $\mathrm{K}_{3} \mathrm{Fe}(\mathrm{CN})_{6}(1.5 \mathrm{mM})(\Delta)$ or p-benzoquinone $(3 \mathrm{mM})(0)$. 


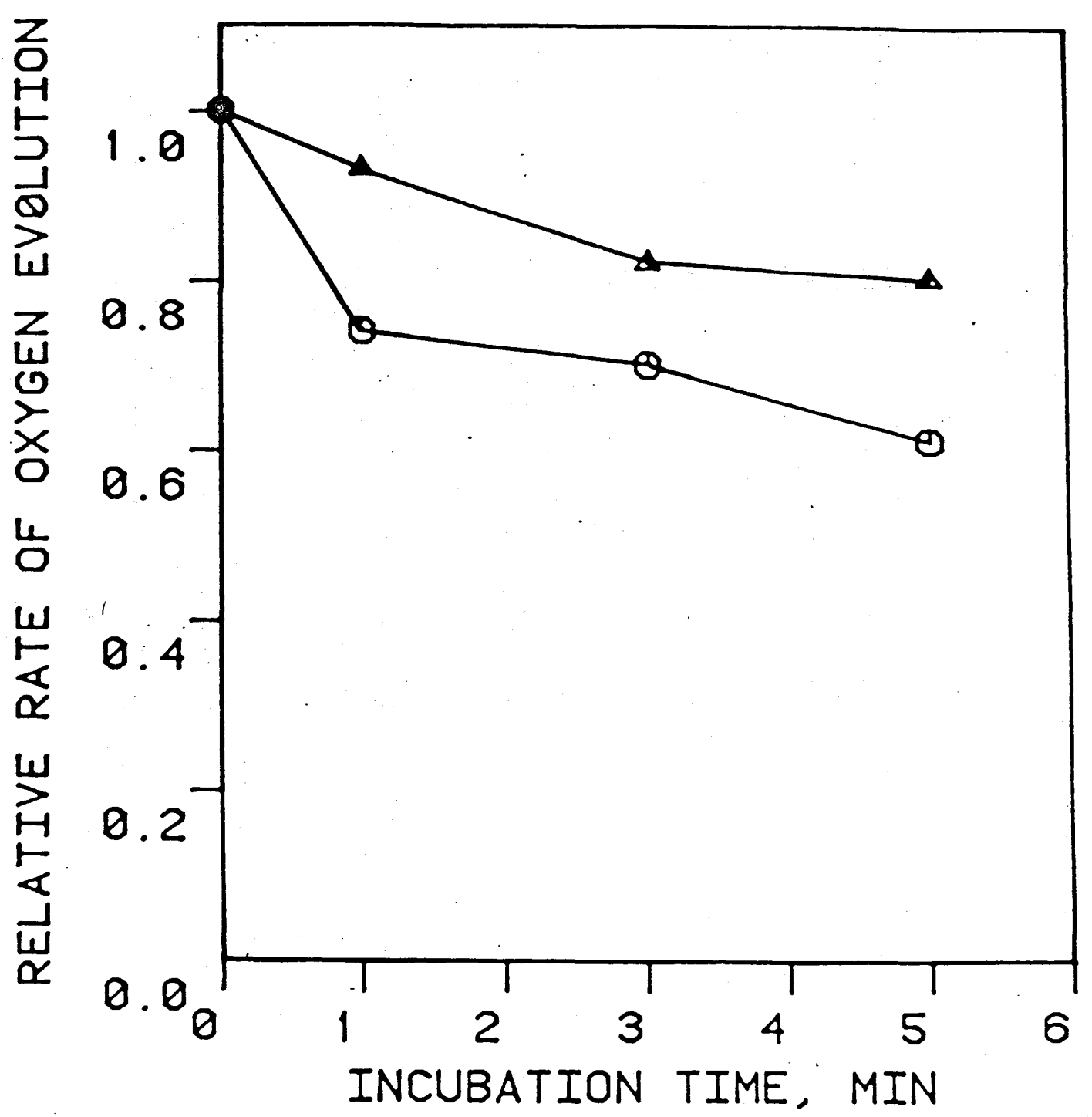

FIGURE 62 .

The effect of low concentrations of chymotrypsin on the rates of oxygen evolution in uncoupled thylakolds. Thylakolds with a chlorophyll concentration of $50 \mu \mathrm{g} \mathrm{ml^{-1 }}$ were treated with chymotrypsin $\left(10 \mu \mathrm{g} \mathrm{ml^{-1 }}\right)$ In a medium containing $20 \mathrm{mM}$ Triclne, $10 \mathrm{mM} \mathrm{KCl}$ and $2 \mathrm{mM} \mathrm{MgCl}_{2}, \mathrm{pH}$ 7.4. The enzyme reaction was stopped by adding trypsin inhibitor (.15 $\mathrm{mg} \mathrm{ml^{-1 }}$ ). Measurements of oxygen evolution were made using $\mathrm{K}_{3} \mathrm{Fe}(\mathrm{CN})_{6}$ $(1.5 \mathrm{mM})(\Delta)$ or p-benzoquinone $(3 \mathrm{mM})(0)$. 
These results show that at low concentrations and short incubation times trypsin $\left(5 \mu \mathrm{g} \mathrm{ml}{ }^{-1}\right)$ and chymotrypsin $\left(10 \mu \mathrm{g} \mathrm{ml}^{-1}\right)$ inhibited electron transport.

It would also appear that the rate of electron transport after enzyme treatment was dependent on the type of electron acceptor present. This shows similar trends as the data in chapter 3 and suggest that even at low concentrations these enzymes affect the acceptor side of PSII.

\subsection{DISCUSSION.}

The results in this chapter have shown that trypsin reduced the amplitude of the electrochromic bandshift to a greater extent in thylakoids resuspended in highsalt media compared to similarly treated membranes in low-salt. In the case of chymotrypsin no such difference was evident. Trypsin was also seen to. stimulate the PSI-mediated electric field and inhibit the $\mathrm{Mg}^{2+}$ - induced chlorophyll fluorescence increase. chymotrypsin had little or no effect on these two parameters.

A possible hypothesis to account for these results is that the decrease in the electrochromic bandshift (of both PSII and PSI), brought about by trypsin in high-salt, is due to a decrease in the PSII- induced field caused by a redistriblition of energy in favour of PSI. If under these conditions PSI was limited by the availability of electrons then it would not be able to use this energy and the overall size of the bandshift would decrease. However, addition of exogenous electron donors to PSI would remove this limiting factor;an increase in the PSI-mediated response would be expected. Therefore it is possible that the increase in amplitude of the PSI-induced bandshift signal, seen after trypsin-treatment, is due to removal of a 
portion of LHCP from PSII which has been implicated in the control of energy redistribution between the two photosystems and in the maintenance of granal stacks (see 1.6.2). Support for this proposal comes from the loss of the $\mathrm{Mg}^{2+}$ - induced fluorescence increase and in the electron micrographs showing that granal stacks are lost after trypsin treatment. Further evidence suggesting that trypsin removes a portion of LHCP associated with PSII came from the light-intensity curve data which showed that after trypsin treatment a more intense flash of 1 ight is required to produce the same yield of oxygen.

However, this explanation cannot account for the reduction in amplitude induced by trypsin in lowsalt or by chymotrypsin. Measurements of the flashyield of oxygen as a function of light intensity suggests that chymotrypsin has no effect on the 1ightharvesting pigments associated with PSII. Therefore two possible explanations for this decrease remain: 1) it could be due to a loss of the electrochromic bandshift sensing pigments, as occurs in photosynthetic bacteria (Webster et al, 1980 ); or 2) a direct effect on one or more of the reaction centre polypeptides.

Measurements of chlorophyll fluorescence indicate that neither trypsin (in low-salt) nor chymotrypsin affect the level of $\mathrm{F}_{\mathrm{V}} / \mathrm{F}_{\mathrm{m}}$ which can be used as an indicator of the efficiency of photochemistry in PSII. Therefore it seems unlikely that the reduction in amplitude of the bandshift is due to a direct inhibition of the primary photochemical reactions. This result points the finger even more strongly at a loss of bandshift sensing pigments. However, measurements of steady-state oxygen evolution showed that trypsin $\left(1.5 \mu \mathrm{g} \mathrm{ml}^{-1}, 5 \mathrm{~min}\right)$ and chymotrypsin $(5 \mu \mathrm{g} \mathrm{ml}-1,5 \mathrm{~min})$ 
inhibited oxygen evolution. This effect was more marked in the presence of p-benzoquinone compared to potassium ferricyanide. These results show similar trends but are less marked than the effects seen with trypsin (50 $\left.\mathrm{gg} \mathrm{m}^{-1}\right)$ and chymotrypsin $\left(100 \mu \mathrm{g} \mathrm{ml}^{-1}\right)$ in Chapter 3. These results suggest that an effect on the reaction centre polypeptides cannot be ruled out. But there are problems involved with comparing steady-state 1 ight and flash illumination studies. Inhibition of oxygen evolution may be caused by a reduction in the efficiency of secondary electron transfer steps which would not necessarily affect the single-turnover events occurring during bandshift measurements. Therefore this still leaves an effect on the bandshift pigments as a candidate to explain the effects of trypsin (1owsalt) and chymotrypsin on the extent of the bandshift.

This study suggests agair (c.f. Steinback et al 1 1979) that the primary site for trypsin digestion of the membrane is the LHCP of PSII. This effect is observed at very low concentrations and incubation times. The implication of this is that, in order to examine the effects of trypsin on the function of the thylakoid membrane it is virtually impossible to do so without causing membrane destacking which brings along with it a number of functional changes. Therefore it might be useful to use chymotrypsin as a tool to investigate the structure and function of the membrane, particularly when it is desirable to maintain granal stacks.

In fact looking at Figure 55 it would appear that chymotrypsin induces tighter stacking compared to the control. The possibility exists that the differential effect of these enzymes may also be due to problems of accessibility. It is clear from the data in this Chapter that trypsin is always actingupon a destacked system whilst chymotrypsin is not. However the results in Figure 53 isuggest that this is not the case:chymotrypsin is shown to have a similar. effect on the electrochromic bandshift of thylakoids treated in the presence or absence of cations. 
CHAPTER 7 - DISCUSISION. 


\section{DISCUSSION.}

\subsection{INTRODUCTION.}

In this thesis a comparison has been made between the effects of two proteolytic enzymes, trypsin and chymotrypsin: on the functions of the thylakoid membrane. These two enzymes attack bonds between different specific amino acids. The intention of this work was to look at the changes in function induced by trypsin and chymotrypsin and to see if it was possible to distinguish differential action, which may be correlated with the availability of particular amino acid bonds at the outer surface of the membrane.

The results have shown that under some circumstances differences in the effects of trypsin and chymotrypsin were evident. In this concluding section these effects will be discussed with an emphasis on the differential action.

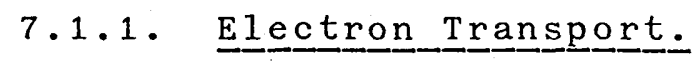

Trypsin and chymotrypsin were shown to have similar effects on uncoupled electron transport (Chapter 3 Figs 16-21).

These results agreed with the work of Renger et al (1976) for trypsin and Zhang et a I (1980) for chymotrypsin. However, using much lower enzyme concentrations, a difference between the effects of these enzymes was observed. Trypsin, and to a lesser extent chymotrypsin, stimulated the rate of electron transport in coupled thylakoids. Trypsin had a marked stimulatory effect on both dark- and 1ight-treated membranes. However, in the case of chymotrypsin a small but significant stimulation of electron transport was seen in lighttreated samples whilst in similar dark-treated membranes little or no effect was observed. 
These results suggested that trypsin and chymotrypsin had an uncoupling effect on thylakoid membranes in agreeance with the results of selman and Bannister (1973).

\subsubsection{The ATPase Complex}

The results in Chapter 4 support the proposal made in 7.2.1 that trypsin and chymotrypsin tend to uncouple the thylakoid membrane. Moreover it was shown that this effect was most likely due to partial digestion of the ATPase complex. Trypsin and chymotrypsin were shown to affect both functions of this complex: 1) phosphorylation was inhibited and 2) hydrolysis was stimulated. The action of trypsin and chymotrypsin on the hydrolytic function appeared to be essentially the same (chapter 4 Tables 5 and 6 ). However examining the data more closely some of the results suggest that the action of trypsin and chymotrypsin on the phosphorylation "reactions of the ATPase, complex was different.

1. Trypsin was seen to have a more marked effect on the half-time of decay of the electrochromic bandshift of light-compared to dark-treated membranes. But in the case of chymotrypsin the half-time of decay of the bandshift was slightly longer in light- compared to darktreated samples.

2. Comparing the phosphorylation data (chapter 4, Tables, 7 and 8 ) with the oxygen evolution data (Chapter 3 , Figs. 18 and 19) it can be seen that trypsin stimulated the rate of oxygen evolution ( $\mathrm{H}_{2} \mathrm{O}$ to potassium ferricyanide) under conditions where phosphorylation was inhibited. In the case of chymotrypsin this correlation is not as clear and in dark- treated membranes phosphorylation was markedly decreased whilst no stimulation of oxygen evolution was evident. 
These two observatons suggested that trypsin had a more marked uncoupling effect than chymotrypsin. But more importantly these results make it possible to propose that, in the case of trypsin, inhibition of phosphorylation is due entirely to uncoupling of electron transport caused by an increase in the permeability of the membrane.

In contrast it is proposed that at least two factors are involved in the chymotrypsin - induced reduction in the rate of phosphorylation; one may be due to uncoupling of the membrane in a similar manner as trypsin but in addition chymotrypsin may directly affect the catalytic reaction thus inhibiting phosphorylation without stimulating oxygen evolution.

These differences may be attributed to different sites on the ATPase complex being sensitive to each enzyme: if for example trypsin digested the $Y$ sub-unit (Moróney and McCarty, 1982) whilst chymotrypsin digested both the $Y$ and $\boldsymbol{\beta}$ sub-unit this might explain the above results. However, the PAGE analysis in this thesis did not support the data of Moroney and McCarty (1982) and in addition it suggested that both trypsin and chymotrypsin affected $\boldsymbol{\beta}$ sub-unit.

Another important difference between the effects of these enzymes was that, after incubation in the light with trypsin,phosphorylation and oxygen evolution (from $\mathrm{H}_{2} \mathrm{O}$ to methyl viologen) were stimulated quite markedly (chapter 4, Table 8). In the case of chymotrypsin-treated samples no stimulation occurred under the conditions investigated.

This stimulatory effect of trypsin could be due to digestion of the $\varepsilon$ sub-unit believed to be the natural ATPase inhibitor which in the solubilised enzyme, is known to be sensitive to trypsin (Nelson et al 1972 ). 
Harris and crofts (1978) suggested that initiation of phosphorylation by light was dependent on the reversible detachment of an inhibitor polypeptide of the ATPase complex. However, the data of Mor oney and McCarty (1982) and the PAGE analysis data presented here do not support this. In addition Finel et al (1984) suggested that trypsin still had the ability to further stimulate ATPase activity in membranes from which the $\varepsilon$ sub-unit is absent. Nelson et al (1973) suggested that there is an interaction between the $\gamma$ and $\varepsilon$ sub-units therefore it is possible that digestion of the $Y$ sub-unit by trypsin may by pass the $\varepsilon$ - controlled activation step.

7.1.3. PSII-Mediated Reactions.

The extent of the electrochromic bandshift was reduced by trypsin and to a lesser degree by chymotrypsin. It was shown (chapter 6) that trypsin had a more marked effect in samples treated in high-salt than in similar low-salt samples. In contrast, chymotrypsin had a similar effect in both media. It is suggested that the large reduction in the extent of the bandshift induced by trypsin, in chloroplasts resuspended in high salt, was in part due to the removal of a part of the LHCP leading to a reduced excitation of PSII and enhanced excitation of PSI. These data indicated that chymotrypsin had no effect on the light harvesting pigments of PSII and this was supported by measurements of the flash yield of oxygen versus 1 ight intensity.

The reason for the effect of trypsin in low-salt and chymotrypsin on the extent of the electrochromic bandshift has still not been elucidated. However, it appears that only two possible sites of action remain 
1) the reaction centre polypeptides;or 2) the pigments in the membrane which sense the electrochromic bandshift. Both hypotheses lead to further lines of experimentation because: 1)al though the polypéptides of PSII have been identified the function of these polypeptides are still unclear; 2) the pigment/proteins involved in the indication of the bandshift have not been identified. These enzymes may therefore prove useful in helping to solve at least one of these problems.

\subsection{Conclusions.}

In conclusion it can be said that the work presented here has shown that in spite of the different specificities of the two enzymes used, in both cases, the differences in functional changes they induced were subtle. At high enzyme concentrations these differences could easily have been missed. But by using the minimum amount of enzyme necessary to get a change in function the differences in effects emerged. In addition, the results presented here indicate that aside from the availability of the amino-acid konds being important in deciding functional changes the susceptibility of the functions of the protein to conformational changes also plays a large role. This factor was particularly evident in the experiments involving the binding of DCMU to the acceptor side of PSII: it can be seen that changes in amino acid bonds occurring in positions distant from the binding site may still cause conformational changes which would alter the shape of this site thus preventing the binding of the inhibitor. 
The differences in effects of trypsin and chymotrypisin on the ATPase has important implications despite intensive research it is still not clear exactly how the sub-units of this complex interact. If it can be shown that the sub-units affected by trypsin and chymotrypsin are different then this might yield information on the functions of this complex. 
7.2.1 Summary Table

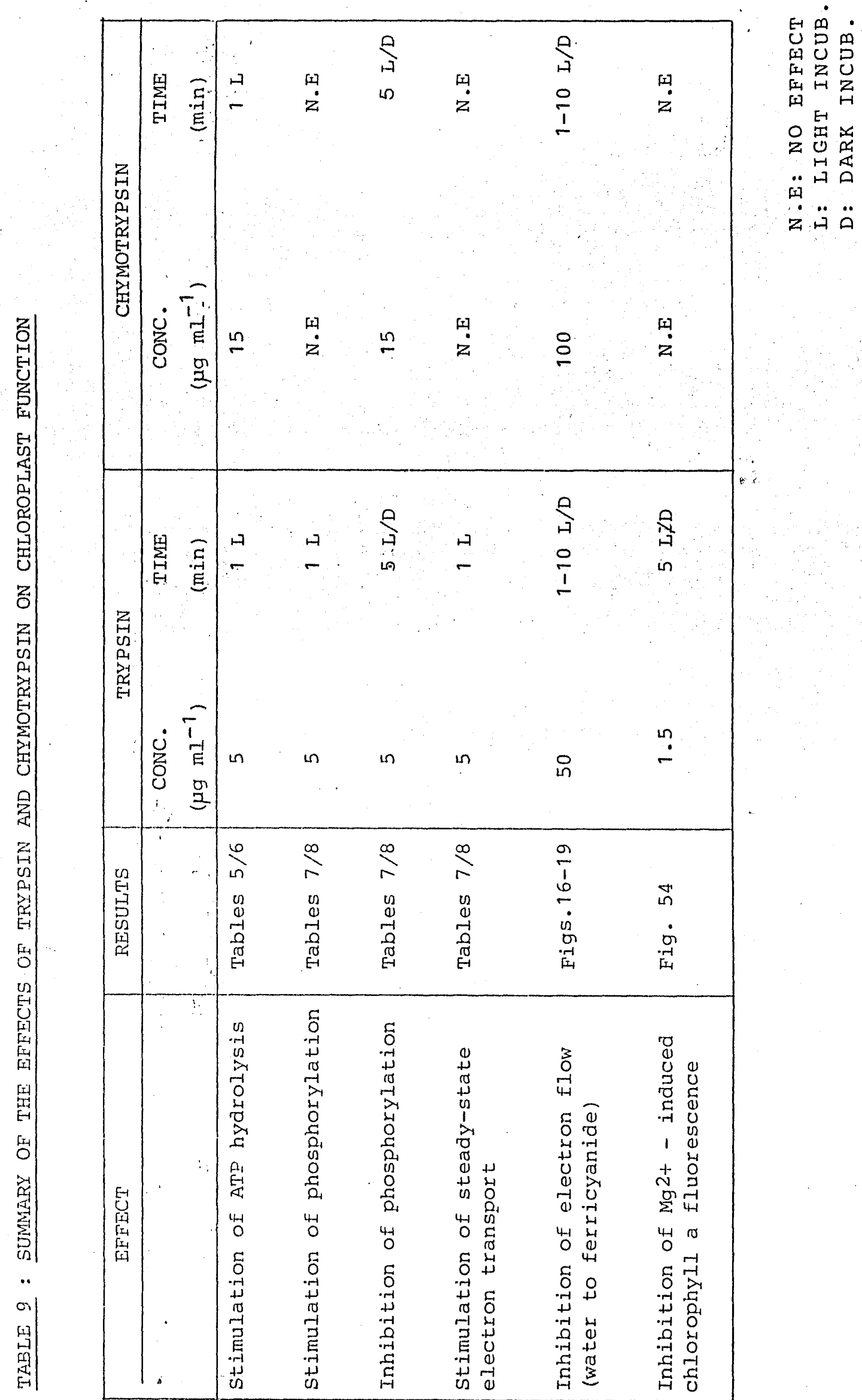


Abramowicz, D.A. and Dismukes, C.G. (1984) Manganese proteins isolated from spinach thylakoid membranes and their role in $\mathrm{O}_{2}$ evolution. II. A binuclear manganese containing 34 kilodalton protein, a probable component of the water dehydrogenase enzyme. Biochim.

Biophys. Acta 765, 318-328

Admon, A., Shahak, Y. and Auran, M. (1982) Adenosine triphosphategenerated transmembrane potential in chloroplasts. Biochim. Biophys. Acta $681,405-411$

Akerlund, H.-E. and Jansson, C. (1981) Localisation of a 34000 and $23000 \mathrm{M}_{\mathrm{r}}$ polypeptide to the lumenal side of the thylakoid membranes. FEBS Lett. 124, 229-232

Akerlund, H.-E., Andersson, B. and Albertsson, P.-A. (1976) Isolation of photosystem II enriched membrane vesicles from spinach chloroplasts by phase partition. Biochim. Biophys. Acta 449, 525-535

Allen, J.F. (1983) Regulation of photosynthetic phosphorylation. Critical Reviews in Plant Science $1,1-23$

Allen, J.F., Bennett, J., Steinbeck, K.E. and Arntzen, C.J. (1981) Chloroplast protein phosphorylation couples plastoquinone redox state to distribution of excitation energy between photosystems. Nature (London) 291, 25-29

Amesz, J. (1983) The role of manganese in photosynthetic $\mathrm{O}_{2}$ evolution. Biochim. Biophys. Acta 726, 1-12

Anderson, J.M. (1975) The molecular organisation of chloroplast thylakoids. Biochim. Biophys. Acta 416, 191-235

Anderson, J.M. (1984) Molecular organisation of chloroplast thylakoid membranes. In: Advances in Photosynthesis Research (Sybesma, C., ed.), Vol. III, pp.1-10, Martinus Nijhoff/Dr.W. Junk, The Hague

Anderson, J.M. and Andersson, B. (1982) The architecture of photosynthetic membranes; lateral and transverse organisation. Trends Biochem. Sci. 7, 288-292

Andersson, B. and Akerlund, H.-E. (1978) Inside-out membrane vesicles isolated from spinach thylakoids. Biochim. Biophys. Acta 503, 462-472 
Andersson, B., Akerlund, H.-E. and Albertsson, P.-A. (1981) Isolation and studies on inside-out thylakoid vesicles. In: Photosynthesis: Structure and Molecular Organisation of the Photosynthetic Apparatus (Akoyunoglou, G., ed.), Vol. III, pp.55-66, Balaban, Philadelphia

Andersson, B., Anderson, J.M. and Ryrie, I.J. (1982) Transbilayer organisation of the chlorophyl1-proteins of spinach thylakoids. Eur. J. Biochem. 123, 465-472

Argyroudi-Akoyunoglou, J.H. and Akoyunoglou, G. (1977) Correlation between cation-induced formation of heavy subchloroplast fractions and the cation-induced increase in chlorophyll a fluorescence yield in tricine-washed chloroplasts. Arch. Biochem. Biophys. 179, 370-377

Arnon, D.I. (1949) Copper enzymes in isolated chloroplasts. Polyphenoloxidase in Beta vulgaris. Plant Phys. 24, 1-15

Arnon, D.I., Allen, M.B. and Whatley, F.R. (1954) Photosynthesis by isolated chloroplasts. Nature (London) 174, 394-396

Arnon, D.I., Tsujimoto, H.Y., McSwain, B.D. (1967) Ferredoxin and photosynthetic phosphorylation. Nature (London) 214, 562-566

Arnon, D.I., Tsujimoto, H.Y. and Tang, G.M.-S. (1981) Proton transport in photo-oxidation of water: a new perspective on photosynthesis. Proc. Nat1. Acad. Sci. U.S.A. 78, 2942-2946

Arntzen, C.J. (1978) Dynamic structural features of chloroplast lamellae. In: Current Tolpics in Bioenergetics (Sanadi, D.R., ed.), Vo1. 8, p'p.111-160, Academic Press, New York

Babcock, G.T. and Sauer, K. (1973) Electron paramagnetic resonance signal II in spinach chloroplasts. I. Kinetic analysis for untreated chloroplasts. Biochim. Biophys. Acta 325, 483-503

Babcock, G.T., Ghanotakis, D.F., Ke, B. and Diner, B.A. (1983) Electron donation to Photosystem II in reaction centre preparations. Biochim. Biophys. Acta $\underline{723}, 276-286$

Baker, N.R. and Horton, P. (1980) Observation of two quenchers of chlorophyll fluorescence in chloroplasts at $196^{\circ} \mathrm{C}$. Biochim. Biophys. Acta 592, 559-564

Bakker-Grunwald, T. and Van Dam, K. (1974) Mechanism of activation of the ATPase in chloroplasts. Biochim. Biophys. Acta 347, 290-298 
Baltimore, B.G. and Malkin, R. (1980a) On the nature of the intermediate electron acceptor (Al) in the photosystem I reaction centre. Photochem. Photobiol. 31, 485-490

Baltimore, B.G. and Malkin, R. (1980b) Spectral characterisation of the intermediate electron acceptor (A1) of photosystem I. FEBS Lett. $110,50-52$

Barber, J. (1976) In: Topics in Photosynthesis: The Intact Chloroplast (Barber, J., ed.), Vol. I, pp.89-134. Elsevier, Holland

Barber, J. (1980) An explanation for the relationship between salt-induced thylakoid stacking and the chlorophyll fluorescence changes assocated with changes in spill over of energy from photosystem II to photosystem I. FEBS Lett. 118, 1-10

Barber, J. (1982) Influence of surface charges on thylakoid structure and function. Ann. Rev. Plant Phys. 33, 261-295

Barber, J. (1984) Lateral hetrerogeneity of proteins and lipids in the thylakoid membrane and implications for electron transport. In: Advances in Photosynthesis Research (Sybesma, C., ed.), Vol. III, pp.91-98, Martinus Nijhoff/Dr. W. Junk, The Hague

Bashford, C.L., Chance, B. and Prince, R.C. (1979a) Oxonol dyes as monitors of membrane potential. Their behaviour in photosynthetic bacteria. Biochim. Biophys. Acta 545, 46-57

Bashford, C.L., Chance, B., Smith, J.C. and Yoshida, T. (1979b) The behaviour of oxonol dyes in phospholipid dispersions. Biophys. J. 25, 63-85

Bassham, J.A., Benson, A.A., Kay, L.D., Harris, A.Z., Wilson, A.T. and Calvin, M. (1954) Cyclic regeneration of carbon dioxide acceptor. Fedn. Proc. Fedn. Am. Socs. Exp. Biol. 13, 1760-1770

Bendal1, D.S. (1982) Photosynthetic cytochromes of oxygenic organisms. Biochim. Biophys. Acta $683,119-151$

Bengis, C. and Nelson, N. (1975) Purification and properties of photosystem I reaction centres from chloroplasts. J. Biol. Chem. 250, 2783-2788

Bennett, J. and Allen, J. (1981) Thylakoid protein phosphorylation: a regulatory role in photosynthesis. In: Photosynthesis: Structure and Molecular Organisation of the Photosynthetic Apparatus (Akoyunoglou, G., ed.), Vol. III, pp.617-622, Balaban, Philadelphia 
Bennoun, P. (1970) Reoxidation of the fluorescence quencher ' $Q$ ' in the presence of 3-(3,4-dichlorophenyl)-1,1 dimethylurea. Biochim. Biophys. Acta 216, 357-363

Berthold, D.A., Babcock, G.T. and Yocum, C.F. (1981) A highly resolved $\mathrm{O}_{2}$-evolving photosystem II preparation from spinach thylakoids. EPR and electron-transport properties. FEBS Lett. 134, 231-234

Boardman, N.K., Anderson, J.M. (1964) Isolation from spinach chloroplasts of particles containing different proportions of chlorophyll a and chlorophyll $b$ and their possible role in the light reactions of photosynthesis. Nature (London) 203, 166-167

Bohme, H. and Cramer, W.A. (1972) The role of cytochrome $\underline{b}_{6}$ in cyclic electron transport: Evidence for an energy coupling site in the pathway of cytochrome $\underline{b}_{6}$ oxidation in spinach chloroplasts. Biochim. Biophys. Acta 283, 302-315

Bose, S. and Arntzen, C.J. (1978) Reversible inactivation of photosystem II reaction centres in cation depleted chloroplast membranes. Arch. Biochem. Biophys. 185, 567-575

Bouges-Bocquet, B. (1973) Electron transfer between the two photosystems in spinach chloroplasts. Biochim. Biophys. Acta 314, 250-256

* Boyer, P.D. (1965) In: Oxidases and related redox systems (King, T.E., Mason, H.S. and Morrison, M., ed.), pp.994-1008, Wiley, New York

Boyer, P.D. (1977) Coupling mechanisms in capture, transmission and use of energy. Ann. Rev. Biochen. 46, 957-966

Briantais, J.M., Vernotte, C., Olive, J. and Wollman, F.A. (1984) Kinetics of cation-induced changes of photosystems II fluorescence and of lateral distribution of the two photosystems in the thylakoid membranes of pea chloroplasts. Biochim. Biophys. Acta 766, 1-8

Burke, J.J., Ditto, C.L. and Arntzen, C.J. (1978) Involvement of the light harvesting complex in cation regulation of excitation energy distribution in chloroplasts. Arch. Biochem. Biophys. 187, 252-263

Butler, W.L. (1978) On the role of cytochrome b 559 in oxygen evolution in photosynthesis. FEBS Lett. $\underline{95}$, 19-25

Calvin, M. (1983) Artificial photosynthesis: quantum capture and energy storage. Photochem. Photobiol. 37, 349-360 
Carlier, M.F. and Hammes, G.G. (1978) Interaction of nucleotides with chloroplast coupling factor 1. Biochemistry 18, 3446-3451

Carmeli, C. (1970) Proton translocation Induced by ATPase activity in chloroplasts. FEBS Lett. $\underline{7}, 297-300$

Carter, D.P. and Staehelin, L.A. (1980) Proteolysis of chloroplast thylakoid membranes. 1. Selective degradation of thylakoid pigmentprotein complexes at the outer membrane surface. Arch. Biochem. Biophys. 200, 364-373

Clayton, R.K. (1965) In: Molecular Physics in Photosynthesis, pp.205, Blaisdell Publishing Co., New York

Clayton, R.K. (1980) In: Photosynthesis: Physical Mechanisms and Chemical Patterns, Cambridge University Press

Colman, P.M., Freeman, H.C., Guss, J.H., Murata, M., Norris, V.A., Ramshaw, J.A.M. and Venkatappea, M.P. (1978) X-ray crystal structure analysis of plastocyanin at $2.7 \AA$ resolution. Nature (London) 272, $319-324$

Cox, R.P. and Anderson, J.M. (1981) Lateral and transverse organisation of cytochromes in the chloroplast thylakoid membrane. Biochem. Biophys. Res. Commun. 103, 1336-1342

Cramer, W.A. and Butler, W.L. (1969) Potentiometric titration of the fluorescence yield of spinach chloroplasts. Biochim. Biophys. Acta $172,503-510$

Cramer, W.A. and Horton, P. (1975) Recent studies on the chloroplast cytocrome b-559. Photochem. Photobiol. 22, 304-308

Crofts, A.R. and Wraight, C.A. (1983) The electrochemical domain of photosynthesis. Biochim. Biophys. Acta 726, 149-185

Crowther, D. and Hind, G. (1980) Partial characterization of cyclic electron transport in intact chloroplasts. Arch. Biochem. Biophys. 204, 568-577

Crowther, D., Mills, J.D. and Hind, G. (1979) Proton motive cyclic electron flow around photosystem $\mathrm{I}$ in intact chloroplasts. FEBS Lett. $98,386-390$

Danks, S.M., Evans, H.E. and Whittaker, P.A. (1983) In: Photosynthetic Systems: Structure, Function and Assembly, J. Wiley \& Sons, New York 
Davis, M.S., Forman, A. and Fajer, J. (1979) Ligated chlorophyll cation radicals: Their function in photosystem II of plant photosynthesis.

Proc. Natl. Acad. Sci. U.S.A. 76, 4170-4174

de Grooth, B.G., Van Gorkom, H.J. and Melburg, R.F. (1980) Generation of the $518 \mathrm{~nm}$ absorption change in chloroplasts by an externally applied field. FEBS Lett. 113, 21-24

Delieu, T. and Walker, D.A. (1972) An improved cathode for the measurement of photosysnthetic oxygen evolution by isolated chloroplasts. New Phytol. 71, 201-225

Doring, G., Stieh1, H.H. and Witt, H. (1967) A second chlorophyll reaction in the electron chain of photosynthesis - registration by the repetitive excitation technique. Z. Naturforsch. 22b, 639-644

Dunahay, T.G., Staehelin, L.A., Seibert, M., Ogilvie, P.D. and Berg, S.P. (1984) Structural, biochemical and biophysical characterisation of four oxygen-evolving photosystem II preparations from spinach. Biochim. Biophys. Acta 764, 179-193

Dutton, P.L. and Prince, R.C. (1978) Equilibrium and disequilibrium in the ubiquinone-cytochrome $b-c_{2}$ oxidoreductase of Rhodopseudomonas sphaeroides. FEBS Lett. $\underline{91}, \overline{1} 5=20$

* Duysens, L.N.M. (1952) Transfer of excitation energy in photosynthesis. Thesis, the University of Utrecht, The Netherlands.

* Duysens, L.N.M. and Sweers, H.E. (1963) Mechanism of two photochemical reactions in algae as studied by means of fluorescence. In: Studies on Microalgae and Photosynthetic Bacteria (Japanese Society of Physiology, ed.), pp.353-372, University of Tokyo Press, Tokyo

Duysens, L.N.M., Amesz, J. and Kamp, B.M. (1961) Two photochemical systems in photosynthesis. Nature (London) 190, 510-511

Emerson, R. (1958) The quantum yield of photosynthesis. Ann. Rev. Plant Physiol. 9 , 1-24

Emerson, R. and Arnold, W. (1932) The photochemical reaction in photosynthesis. J. Gen. Physiol. 16, 191-205

Evans, M.C.W., Lord, A.V. and Reeves, S.G. (1974) The detection and characterisation by electron-paramagnetic-resonance spectroscopy of iron-sulphur proteins and other electron transport components in chromatophores from the purple bacteria Chromatium. Biochem.jJ. 138, 177-183 
Finel, M., Rubinstein, M., Pick, U. (1984) Preparation of $\Sigma$-deficient chloroplast $\mathrm{CF}_{1}$ having a high ATPase activity. FEBS Lett. 166, 85-89

Forster, V. and Junge, W. (1985) Stoichiometry and kinetics of proton release upon photosynthetic water oxidation. Photochem. Photobiol. 41, $183-190$

Fowler, C.F. (1977) Proton evolution from Photosystem II stoichiometry and mechanistic considerations. Biochim. Biophys. Acta 462, 414-421

Fowler, C.F. and Kok, B. (1974) Direct observation of a light-induced electric field in chloroplasts. Biochim. Biophys. Acta 357, 308-318

Fowler, C.F. and Kok, B. (1976) Determination of $\mathrm{H}^{+} / \mathrm{e}^{-}$ratios in chloroplasts with flashing 1ight. Biochim. Biophys. Acta 423, 510-523

Fujita, I., Davis, M.S. and Fajer, J. (1978) Anion radicals of pheophytin and chlorophyll a: Their role in primary charge separation in plant photosynthesis. J. Am. Chem. 100, 6280-6282

Galmiche, J.M. and Girault, G. (1981) Interaction of oxonol VI with spinach chloroplasts. Its use to sense the membrane potential. In: Photosynthesis; Photophysical Processes - Membrane Energisation (Akoyunoglou, G., ed.), Vol. I, pp.535-541, Balaban, Philadelphia

Ghanotakis, D.F., Babcock, G.T. and Yocum, C.F. (1984) Calcium reconstitutes high rates of oxygen evolution in polypeptide depleted PSII preparations. FEBS Lett. 167, 127-130

Girault, G. and Galmiche, J.M. (1978) Effect of nucleotides on the potential and $\mathrm{pH}$ changes across the thylakoid membrane in spinach chloroplasts. Biochin. Biophys. Acta 502, 430-444

Goldbeck, J.H. and Kok, B. (1979) Redox titration of electron acceptor $Q$ and the plastoquinone pool in PSII. Biochim. Biophys. Acta $\underline{547}, 347-360$

Goldbeck, J.H., Lien, S. and San Pietro, A. (1977) Isolation and characterisation of a subchloroplast particle enriched in iron-sulphur protein and P700. Arch. Biochem. Biophys. 178, 140-150

Goldbeck J.H., Velthuys, B.R. and Kok, B. (1978) Evidence that the intermediate electron acceptor, $A_{2}$, in photosystem $I$ is a bound iron-sulphur protein. Blochim. Biophys. Acta 504, 226-230 
Graber, P., Schlodder, E.S. and Witt, H.T. (1977) Conformational change of the chloroplast ATPase induced by a transmembrane electric field and its correlation to phosphorylation. Biochim. Biophys. Acta $461,426-440$

Grierson, D. and Covey, S. (1984) In: Plant Molecular Biology, pp.147-159, Blackie, Glasgow and London

Haenel, W., Nairn, J.A., Reisberg, P. and Sauer, K. (1982) Picosecond fluoresecence kinetics and energy transfer in chloroplasts and algae. Biochim. Biophys. Acta $680,161-173$

Hales, B.J. and Das Gupta, H. (1979) Orientation of the bacteriochlorophyll triplet and the primary ubiquinone acceptor of Rhodospirillium rubrum in membrane multilayers by ESR spectroscopy. Biochim. Biophys. Acta 548, 276-286

Harris, D.A. and Crofts, A.R. (1978) The initial stages of photophosphorylation. Studies using excitation by saturating, short flashes of light. Biochim. Biophys. Acta 502, 87-102

Harris, D.A. and Slater, E.C. (1975) Tightly bound nucleotides of the energy-transducing ATPase of chloroplasts and their role in photophosphorylation. Biochim. Biophys. Acta 387, 335-348

Hauska, G., Hurt, E., Gabellini, F. and Lockau, W. (1983) Comparative aspects of quinol-cytochrome $\mathrm{c} / \mathrm{plastocyanin}$ oxidoreductases. Biochim. Biophys. Acta $726,9 \overline{7}-133$

Haworth, P., Watson, J.L. and Arntzen, C.J. (1983) The detection, isolation and characterisation of a LHC which is specifically associated with photosystem I. Biochim. Biophys. Aca 724, 151-158

Hill, R. (1937) Oxygen evolved by isolated chloroplasts. Nature (London) 139, 881-882

Hill, R. and Bendall, F. (1960) The function of the two cytochrome components in chloroplasts: a working hypothesis. Nature (London) $186,136-137$

Hill, R. and Scarsbrick, R. (1951) The haematin compounds of leaves. New Phytol. 50, 98-111

Hind, G. and Jagendorf, A.T. (1965) Light scattering changes associated with the production of a possible intermediate in photophosphorylation. J. Biol. Chem. 240, 3195-3201 
Hipkins, M.F. and Musto, F.W. (1985) The effect of classical uncouplers and potassium cyanide on the field-indicating absorption change in Chlorella emersonif. Photobiochem. Photobiophys. 9, 159-166

Horton, P. and Black, T. (1980) Activation of adenosine $5^{\prime}$ triphosphate-induced quenching of chlorophyll fluorescence by reduced plastoquinone. The basis of state I-state II transitions in chloroplasts. FEBS Lett. 119, 141-144

Horton, P. and Cramer, W.A. (1974) The accessibility of the chloroplast cytochromes $\underline{f}$ and $\underline{b} 559$ to ferricyanide. Biochim. Biophys. Acta $368,348-3 \overline{6} 0$

Hurt, E. and Hauska, G. (1981) A cytochrome $\underline{f} / \underline{b}_{6}$ complex of five polypeptides with plastoquinol-plastocyanin-oxidoreductase activity from spinach chloroplasts. Eur. J. Biochem. 117, 591-599

Itoh, S. and Morita, S. (1982) Decay of membrane potentials under phosphorylating conditions in chloroplasts with in vivo activation ATPase. Biochim. Biophys. Acta 682, 413-449

Itoh, S., Yerkes, C.T., Koike, H., Robinson, H.H. and Crofts, A.R. (1984) Effects of chloride depletion on electron donation from the water-oxidising complex to the photo-system II reaction centre as measured by the micro-second rise of chlorophyll fluorescence in isolated pea chloroplasts. Biochim. Biophys. Acta $\underline{766}, 612-622$

Izawa, S. and Good, N.E. (1966) Effects of salts on electron transport on the conformation of isolated chloroplasts. II. Electron Microscopy. Plant Physiol. 41, 544-552

Izawa, S., Heath, R.L. and Hind, G. (1969) The role of chloride ion in photosynthesis. III. The effect of artificial electron donors upon electron transport. Biochim. Biophys. Acta 180, 388-398

Jackson, J.B. and Crofts, A.R. (1969) The high energy state in chromatophores from Rhodopseudomonas sphaeroides. FEBS Lett. 4 , 185-189

Jagendorf, A.T. and Hind, G. (1963) In: Photosynthetic Mechanisms of Green Plants, Publ. 1145 Natl. Acad. Sci. National Research Council, Washington D.C.

Jagendorf, A.T. and Uribe, E. (1966) ATP formation caused by acid-base transition of spinach chloroplasts. Proc. Natl. Acad. Sci. U.S.A. 55, 170-177 
Jennings, R.C., Forti, G., Gerola, P.D., Garlaschi, F.M. (1978)

Studies on cation induced thylakoid membrane stacking, fluorescence yield and photochemical efficiency. Plant Phys. 62, 879-884

Jennings, R.C., Garlaschi, F.M., Paolo, D.G. and Fiorti, G. (1979) Partition zone penetration by chymotripsin and the localisation of the chloroplast flavoprotein and photosystem II. Biochim. Biophys. Acta 546, 207-219

Johnson, H.D., Pfister, V.R. and Homann, P.J. (1983) Metastable proton pools in thylakoids and their importance for the stability of photosystem II. Biochim. Biophys. Acta 723, 256-265

* Joliot, P. (1968) Kinetic studies of PSII in photosynthesis. Photochem. Photobiol. 8 , 451-463

Joliot, P. and Delosme, R. (1974) Flash-induced 519nm absorption changes in green algae. Biochim. Biophys. Acta 357, 267-284

Joliot, P. and Joliot, A. (1968) A polarographic method for detection of oxygen production and reduction of hill reagent by isolated chloroplasts. Biochim. Biophys. Acta 153, 625-634

Joliot, P. and Joliot, A. (1984) Electron transfer between the two photo-systems. 1. Flash excitation under oxidising conditions. Biochim. Biophys. Acta 765, 210-218

* Joliot, P., Barbieri, G. and Chaubaud, R. (1969) Un nouveau modele des centres photo-chimique de system II. Photochem. Photobiol. 10, 309-329

Junge, W. (1977) Membrane potentials in photosynthesis. Ann. Rev. Plant Phys. 28, 503-536

Junge, W. and Auslander, W. (1978) Proton release during photosynthetic water oxidation: Kinetics under flashing light. In: Photosynthetic Oxygen Evolution (Metzner, H., ed.), pp.213-228, Academic Press, London

Junge, W. and Witt, H. (1968) On the ion transport system of photosynthesis - investigations on a molecular level. Z. Naturforsch. 23b, 244-254

Jurinsic, P. and Govindjee (1977) Temperature dependence of delayed light emission in the 6 to 340 microsecond range after a single flash in chloroplasts. Photochem. Photobiol. 26, 617-628 
Katoh, S., Suga, I., Shirator, I. and Takamiya, A. (1961)

Distribution of plastocyanin in plants, with special reference to its

localization in chloroplasts. Arch. Biochem. Biophys. 94, 136-141

* Ke, B., Dolan, E., Sughara, K., Hawkridge, F.M., Demeter, S. and Shaw, E.R. (1977) Photosynthetic Organelles: Special issue of Plant and Cell Phys., pp.187-199

Kierns, J.J. and Wang, J.H. (1972) Studies on nicotinamide adenine dinucleotide phosphate reductase of spinach chloroplasts. J. Biol. Chem. 247, 7374-7382

Klimov, V.V., Allakhverdiev, S.I., Demeter, S. and Krasnovsky, A.A. (1979) Photo reduction of pheophytin in the photo-system-2 of chloroplasts depending on the oxidation-reduction potential of the medium. Dokl. Akad. Nauk SSR 249, 227-230

Klimov, V.V., Klevanik, A., Shuvalov, V.A. and Krasnovsky, A.A. (1977) Reduction of pheophytin in the primary light reaction of photosystem II. FEBS Lett. 82, 183-186

Knaff, D.B. and Arnon, D.I. (1969) Spectral evidence for a new photoreactive component of the oxygen-evolving system in photosynthesis. Proc. Nat1. Acad. Sci. U.S.A. 63, 963-969

Kok, B. (1956) On the reversible absorption change at $705 \mu \mathrm{m}$ in photosynthetic organisms. Biochim. Biophys. Acta 22, 399-401

* Kok, B., Forbush, B. and McGloin, M. (1970) Co-operation of charges in photosynthetic oxygen evolution. I - A linear four step mechanism. Photochem. Photobiol. 11, 457-474

Kyle, D. (1985) The 32000 Dalton $Q_{B}$ protein of photosystem II. Photochem. Photobiol. 41 , 107-116

Lam, E., Oritz, W. and Malkin, R. (1984) Ch1 a/b proteins of photosystem I. FEBS Lett. 168, 10-14

Lam, E., Baltimore, B., Oritz, W., Schollar, S., Melts, A. and Malkin, R. (1983) Characterisation of a resolved oxygen-evolving photosystem II preparation from spinach thylakoids. Blochim. Biophys. Acta 224 , 201-211

Lavorel, J. (1978) On the origin of damping of the oxygen yield in sequences of flashes. In: Photosynthetic Oxygen Evolution (Metzner, H., ed.), pp.249-268, Academic Press, London 
Levine, R.P. (1969) The analysis of photosynthesis using mutant stralns of algae and higher plants. Ann. Rev. Plant Physiol. 20, 523-540

Lien, S. and Racker, E. (1971) Preparation and assay of chloroplast coupling factor 1. In: Methods in Enzymology: Photosynthesis Part A (San Pletro, A., ed.), Vol. 23, pp.547-555, Academic Press, New York

Ljungberg, U., Akerlund, H.-E., Andersson, B. (1984) The release of a 10-kDa polypeptide from everted photosystem II thylakoid membranes by alkaline Tris. FEBS Lett. 175, 255-258

Ljungberg, U., Jansson, C., Andersson, B. and Akerlund, H.-E. (1983) Reconstitution of oxygen evolution in high salt washed photosystem II particles. Biochem. Biophys. Res. Common. 113, 738-744

Lynn, W.S. and Straub, K.D. (1969) ADP kinase and ATPase in chloroplasts. Proc. Natl. Acad. Sci, U.S.A. 63, 540-547

McCarty, R.E. (1971) Chloroplast preparations deficient of CF 1 In: Methods in Enzymology: Photosynthesis Part A (San Pietro, A., ed.), Vo1. 23, pp.251-256, Academic Press, New York.

McCarty, R.E. (1978) The ATPase complex of chloroplasts and chromatophores. In: Current Topics in Bioenergetics (Sanadi, D.R. and Vernon, L.P. ed.), Vo1. 7, pp.245-278, Academic Press, New York

McCarty, R.E. and Carmeli, C. (1982) In: Photosynthesis: Energy Conversion by Plants and Bacteria (Govindjee, ed.), Vol. I, pp.647-695, Academic Press, New York

Magnusson, R.P. and McCarty, R.E. (1976) Light-induced exchange of nucleotides into coupling factor 1 in spinach chloroplast thylakoids. J. Biol. Chem. 251, 7417-7422

Malkin, R. (1982) Redox properties and functional aspects of electron carriers in chloroplast photosynthesis. In: Topics in Photosynthesis: Electron Transport and Photophosphorylation (Barber, J., ed.), Vol. 4, pp.2-42, Elsevier, Holland

Malkin, R. and Aparicio, P.J. (1975) Identification of a $g=1.90$ high potential iron-sulphur protein in chloroplasts. Biochem. Blophys. Res. Comm. 63, 1157-1160

Malkin, R. and Bearden, A.J. (1971) Primary reactions of photosynthesis: Photoreduction of a bound chloroplast ferredoxin at low temperature as detected by EPR spectroscopy. Proc. Natl. Acad. Sci. U.S.A. 68, 16-19 
Malkin, R. and Chain, R.K. (1980) The relationship of the cyclic and non-cyclic electron transport pathways in chloroplasts. Biochim. Biophys. Acta 591, 381-390

Malkin, R. and Siderer, Y. (1974) The effect of salt concentration on the fluorescence parameters of isolated chloroplasts. Biochim.

Biophys. Acta $368,422-423$

Mansfield, R.W. and Bendall, D.S. (1984) Cytochrome distribution across chloroplast thylakoid membranes. Controlled proteolysis of inside-out and right-side out vesicles. Biochim. Biophys. Acta 766, 62-69

Mantai, K.E. (1969) Carbamyl m-chlorophenylhydrazone as an inhibitor of coupled electron transport in trypsin treated spinach chloroplasts. Biochim. Biophys. Acta 189, 449-451

Manta1, K.E. (1970) Some effects of hydrolytic enzymes on coupled and uncoupled electron flow in chloroplasts. Plant Physiol. 45, 563-566

Marsho, T.V. and Kok, B. (1970) Interaction between electron transport components in chloroplasts. Biochim. Biophys. Acta 223, 240-250

Melis, A. and Brown, J.S. (1980) Stoichiometry of system I and system II reaction centres and of plastoquinone in different photosynthetic membranes. Proc. Natl. Acad. Sci. U.S.A. 77, 4712-4716

Miller, K.W. and Staehlein, L. (1976) Analysis of the thylakoid outer surface. Coupling factor is limited to unstacked regions. J. Cell Biol. $68,30-47$.

Mills, J.D. and Hind, G. (1979) Light-induced $\mathrm{Mg}^{2+}$ ATPase activity of coupling factor in intact chloroplasts. Biochim. Biophys. Acta 547, 455-462

Mills, J.D. and Mitchell, P. (1982) Thiol modulation of $\mathrm{CF}_{0}-\mathrm{CF}_{1}$ stimulates acid/base dependent phosphorylation of ADP of broken pea chloroplasts. FEBS Lett. 144, 63-67

Mills, J.D., Mitchell, P. and Schurmann, P. (1980) Modulation of coupling factor ATPase activity in intact chloroplasts. The role of the thioredoxin system. FEBS Lett. 112, 173-177

Mills, J.D., Crowther, D., Slovacek, R.E., Hind, G. and McCarty, R.E. (1979) Electron transport pathways in spinach chloroplasts. Reduction of the primary acceptor of photosystem II be reduced nicotinamide adenine dinucleotide phosphate in the dark. Biochim. Biophys. Acta 547, 127-137 
Mitchell, P. (1961) Coupling of phosphorylation to electron and hydrogen transfer by a chemi-osmotic type of mechanism. Nature (London) 191, 144-148

Mitchell, P. (1974) A chemiosmotic molecular mechanism for proton-translocating adenosine triphosphates. FEBS Lett. 43 , 189-194

Mitchel1, P. (1976) Possible molecular mechanisms of the protonmotive function of cytochrome systems. J. Theor. Biol. 62 , 327-367

Mitche11, P. (1985) Molecular mechanics of proton motive $F_{0} F_{1}$ ATPases. Rolling well and turnstile hypothesis. FEBS Lett. 182, 1-7

Miyao, M. and Murata, N. (1983) Partial disintegration and reconstitution of the photosynthetic $\mathrm{O}_{2}$ evolution system - binding of $24 \mathrm{kDa}$ and $18 \mathrm{kDa}$ polypeptides. Biochim. Biophys. Acta 725, 87-93

Miyao, M. and Murata, N. (1984) Role of the $33 \mathrm{kDa}$ polypeptide in preserving $\mathrm{Mn}$ in the photosynthetic oxygen-evolution system and its replacement by chloride ions. FEBS Lett. 170, 350-354

Miyao, M. and Murata, N. (1985) The $\mathrm{Cl}^{-}$effect on photosynthetic oxygen evolution: Interaction of $\mathrm{Cl}^{-}$with $18 \mathrm{kDa}$ and $33 \mathrm{kDa}$ proteins. FEBS Lett. 180, 303-308

Moroney, J.V. and McCarty, R.E. (1982) Light dependent cleavage of the sub-unit of $\mathrm{CF}_{1}$ by trypsin causes activation of $\mathrm{Mg}^{2+}$ ATPase activity and uncoupling of photophosphorylation in spinach chloroplasts. J. Biol. Chem. 257, 5915-5920

Morschel, E. and Staehelin, A.L. (1983) Reconstitution of cytochrome $-\underline{f} / \underline{b} 6$ and $\mathrm{CF}_{0}-\mathrm{CF}_{1}$ ATP synthetase complexes into galactolipid 1iposomes. J. Cell Biol. 97, 301-310

Moss, D.A. and Bendall, D.S. (1984) Cyclic electron transport in chloroplasts. The Q-cycle and the site of action of antimycin. Biochim. Biophys. Acta 767, 389-395

Mullet, J.E. and Arntzen, C.J. (1981) Identification of a 32-34 kilodalton polypeptide as a herbicide receptor protein in photosystem II. Biochim. Biophys. Acta 635, 236-248

Mullet, J.E., Baldwin, T.0. and Arntzen, C.J. (1981) A mechanism for chloroplast thylakoid adhesion mediated by the ChI a/b light harvesting complex. In: Photosynthesis: Structure and Molecular Organisation of the Photosynthetic Apparatus (Akoyunoglou, G., ed.), Vol. III, pp.557-582, Balaban, Philadelphia 
Mullet, J.E., Burke, J.J. and Arntzen, C.J. (1980) Chlorophyl1 proteins of photosystem I. Plant Physio1. 65, 814-822

Nelson, N. (1976) Structure and function of chloropast ATPase. Biochim. Biophys. Acta 456, 314-338

Nelson, N. (1981) Proton-APTase of chloroplasts. In: Current Topics in Bioenergetics (Sanadi, D.R., ed.), Vol. 11, pp.1-33, Academic Press, New York.

Nelson, N. and Neuman, J. (1972) Isolation of a cytochrome $\underline{b}_{6}-\underline{f}$ particle from chloroplasts. J. Biol. Chem. 247, 1817-1824

Nelson, N., Nelson, H. and Racker, E. (1972) Partial resolution of the enzymes catalysing photophosphorylation. XII. Purification and properties of an inhibitor isolated from chloroplast coupling factor 1. J. Biol. Chem. 247, 7657-7662

Nicholls, D.G. (1982) In: Bioenergetics: An Introduction to the Chemiosmotic Theory. Academic Press, London.

Nishimura, M., Ito, T. and Chance, B. (1962) Studies on bacterial photophosphorylation III. A sensitive and rapid method of determination of photophosphorylation. Biochim. Biophys. Acta $\underline{59}$, $177-182$

Norris, J.R., Scheer, H., Druyan, M.E. and Katz, J.J. (1974) An electron-nuclear double resonance (ENDOR) study of the special pair model for photo-reactive chlorophyll in photosynthesis. Proc. Natl. Acad. Sci. U.S.A. 71, 4897-4900

Okamura, M.Y., Feher, G. and Nelson, N. (1982) In: Photosynthesis: Energy conversion by Plants and Bacteria (Govindjee, ed.), Vol. I, pp.195-274, Academic Press, New York

Okayama, S. (1976) Redox potential of plastoquinone A in spinach chloroplasts. Biochim. Biophys. Acta $440,331-336$

Olsen, L.F. and Barber, J. (1981) Origin of the slow component of the electrochromic shift: a charge delocalisation model. FEBS Lett. 123, 90-94

01sen, L.F., Telfer, A. and Barber, J. (1980) A flash spectroscopic study of the kinetics of the electrochromic shift, protein release and redox behaviour of cytochromes $\underline{f}$ and $\underline{b} 563$ during cyclic electron flow. FEBS Lett. 118, 11-17 
O'Malley, P.J. and Babcock, G.T. (1984) EPR properties of immobilised quinone cation radicals and the molecular origin of signal II in spinach chloropalsts. Biochim. Biophys. Acta 765, 370-379

Omata, T., Murata, N. and Satoh, K. (1984) Quinone and pheophytin in the photosynthetic reaction centre II from spinach chloroplasts. Biochim. Biophys. Acta 765, 403-405

Oritz, W. and Malkin, R. (1985) Topographical studies of the polypeptide subunits of the cytochrome $\underline{b}-\underline{\underline{f}}$ complex. Biochim. Biophys. Acta $808,164-170$

Oritz, W., Lam, E., Chollar, S., Munt, D. and Malkin, R. (1985) Topography of the protein complexes of the chloroplast thylakoid membrane. Studies of photosystem I using a chemical probe and proteolytic digestion. Plant Physiol. 77, 389-397

Ort, D.R. and Melandri, B.0. (1982) In: Photosynthesis: Energy Conversion by Plants and Bacteria (Govind jee, ed.), Vol. I, pp.539-587, Academic Press, New York

Peters, R.L.A., Van Kooten, O. and Vredenberg, W.J. (1983) On the correlation between the activity of ATP-hydrolase and the kinetics of the flash-induced P515 in spinach. J. Bioenerg. Biomem. 115, 335-356

Peters, F.A.L.J., Van Der Pal, R.H.M., Peters, R.L.A., Vredenberg, Wim. J. and Kraayenhof, R. (1984) Studies on well coupled PSI-enriched subchloroplast vesicles. Discrimination of flash-induced fast electric potential components. Biochim. Biophys. Acta $\underline{766}$, 169-178

Petrack, B., Cranston, A., Sheppy, F., Farron, F. (1965) Studies on the hydrolysis of adenosine triphosphate by spinach chloroplasts. J. Biol. Chem. 240, 906-912

Pfister, K. and Arntzen, C.J. (1979) The mode of action of photosystem II - specific inhibitors in herbicide-resistant weed biotypes. Z. Naturforsch. 34C, 996-1009

Pfister, K., Steinback, K.E., Gardner, G. and Arntzen, C.J. (1981) Photoaffinity labelling of a herbicide receptor protein in chloroplast membranes. Proc. Natl. Acad. Sci. U.S.A. 78; 981-985

Pirson, A. (1937) Ernahrungs - und stoffweschel physiologische untersuchungen an Fontinalis and Chlorella. Z. Bot. 31, 193-267

Platt, J.R. (1961) Electrochroism, a possible change of colour producible in dyes by an electric field. J. Chem. Phys. 34, 8962-863 
Prince, R.C., Lindsay, J.G. and Dutton, P.L. (1975) The Rleske iron-sulphur centre in mitochondrial and photosynthetic systems: $\mathrm{E}_{\mathrm{m}} / \mathrm{pH}$ relationships. FEBS Lett. $51,108-111$

Racker, E. (1977) Mechanisms of energy transformations. Ann. Rev. Biochem. 46, 1006-1024

Rathenow, M. and Rumberg, B. (1980) Stoichiometry of proton translocation during photosynthesis. Ber. Bunsenges. Phys. Chem. 84 , 1059-1062

Regitz, G. and Ohad, I. (1976) Trypsin-sensitive photosynthetic activities in chloroplast membranes from Chlamydomonas reinhardii, y - 1. J. Biol. Chem. 251, 247-252

Reich, R. and Siewe, K.-U. (1977) The effect of molecular organisation on the electrochroism of carotenoids. 1. The influence of the carboxylic group. Photochem. Photobiol. 26, 11-17

Relske, J.S. (1976) Composition, structure and function of complex III of the respiratory chain. Biochim. Biophys. Acta 456, 195-247

Reiske, J.S., MacLennan, D.H. and Coleman, R. (1964) Isolation and properties of an iron-sulphur protein from the (reduced coenzyme Q) cytochrome $c$ reductase complex of the respiratory chain. Biochem. Biophys. Res. Commun. 15, 338-344

Renger, G. (1976) The use of trypsin as a structurally selective inhibitor at the outer surface of the thylakoid membrane. Biochim. Biophys. Acta 440, 287-300

Renger, G., Hagemann, R. and Dohnt, G. (1981) Properties of the proteinaceous component acting as apoenzyme for the functional plastoquinone groups on the acceptor side of system II. Biochim. Biophys. Acta $636,17-26$

Renger, G., Hagemann, R. and Dohnt, G. (1983) The role of lysine residues for excitation energy tranfer and herbicide action in system II. Photochem. Photobiophys. $\underline{5}, 273-279$

Renger, G., Erixon, K., Doring, G., Wolff, Ch. (1976) Studies on the nature of the inhibitory effect of trypsin on the photosynthetic electron transport of system II in spinach chloroplasts. Biochim. Biophys. Acta 440, 278-286

Rich, P.R. (1984) Electron and proton transfers through quinones and cyt $\underline{\mathrm{b}}$ c complexes. Biochim. Biophys. Acta $\underline{768}$, 53-79 
Ruben, S., Randa11, M., Kamen, M. and Hyde, J.L. (1941) Heavy oxygen $\left(0^{18}\right)$ as a tracer in the study of photosynthesis. J. Am. Chem. Soc. $\underline{63}, 877-880$

Rumberg, B. and Siggel, U. (1968) Quantitative zusammenhange zwischen chlorophyl1-b - reaktion, elektronentransport und phosphorylierung bier der photosynthese. Z. Naturforsch. Teil B 23, 239-244

Rutherford, A.W., Zimmerman, J.L. and Mathis, P. (1984) EPR of PS II - interactions, herbicide effects and a new signal. In: Advances in Photosynthesis Research (Sybesma, C. ed.), Vol. I, pp.445-448. Martinus Nijhoff/Dr. W. Junk, The Hague

Ryrie, I.J., Anderson, J.M. and Glare, T. (1985) Transmembrane organisation of individual polypeptides of photosystem I complex and the light-harvesting complex of photo-system II of thylakoid membranes. Photobiochem. Photobiophys. 9, 145-157

Sandmann, G., Bohner, H., Bohme, H. and Boger, P. (1981) Plastocyanin and cytochrome $c-553$, two different electron donors to photosystem I in algae. In: Photosynthesis: Photosynthetic Electron Transport and Photophosphorylation (Akoyunoglou, G., ed.), Vol. II, pp.623-632, Balaban, Philadelphia

Sandusky, P.O. and Yocum, C.F. (1984) The chloride requirement for photosynthetic oxygen evolution. Analysis of the effects of chloride on other anions, an inhibition of the oxygen-evolving complex. Biochim. Biophys. Acta 766 , 603-611

Schapendonk, A.H.C.M. and Vredenberg, W.J. (1977) Salt induced absorbance change of P515 in broken chloroplasts. Biochin. Biophys. Acta $462,613-621$

Schapendonk, A.H.C.M. and Vredenberg, W.J. (1979) Activation of the reaction II component of $\mathrm{P} 515$ in chloroplasts by pigment system $I$. FEBS Lett. 106, 257-261

Schapendonk, A.H.C.M., Vredenberg, W.J. and Tonk, W.J.M. (1979) Studies on the kinetics of the $515 \mathrm{~nm}$ absorbance changes in chloroplasts. Evidence for the induction of a fast and slow P515 response upon saturating light flashes. FEBS Lett. 100, 325-329

Schmidt, S., Reich, R., Witt, H.T. (1971) Electrochroism of chlorophylls and carotenoids in multilayers in chloroplasts. Naturwissenschaften $\underline{58}, 414-415$

Schuurmans, J.J. and Kraayenhof, R. (1983) Energy-regulated functional transitions of chloroplast ATPase. Photochem. Photobiol. 37, 85-91 
Schuurmans, J.J., Casey, R.P. and Kraayenhof, R. (1978) Transmembrane electric potential formation in spinach chloroplasts. FEBS Lett. 94, 405-409

Schuurmans, J.J., Leewerik, F.J., Siu Oen, B. and Kraayenhof, R. (1981) Denconvolution of the flash-induced carotenoid and oxonol VI responses in broken chloroplasts. In: Photosyntheses: Photophysical Processes - Membrane Energisation (Akoyunoglou, G., ed.), Vol. I, pp.543-552, Balaban, Philadelphia

Schwert, G.W. and Takenaka, Y. (1955) A spectrophotometric determination of trypsin and chymotrypsin. Biochim. Biophys. Acta 16, 570-575

Selman, B.R., Bannister, T.T. and Dilley, R.A. (1973) Trypsin inhibition of electron transport. Biochim. Biophys. Acta 292, 566-581

Shoshan, V., Shavit, N. and Chipman, D.M. (1978) Kinetics of nucleotide binding to chloroplast coupling factor $\left(\mathrm{CF}_{1}\right)$. Biochim. Biophys. Acta 504, 108-122

Shuvalov, V.A., Dolan, E. and Ke, B. (1979) Spectral and kinetic evidence for two early electron acceptors in photosystem I. Proc. Nat1. Acad. Sci. U.S.A. $\underline{76}, 770-773$

Siggel, U. (1984) The effect of flash-induced ATP-hydrolysis on the 515 absorbance-change. In: Advances in Photosynthesis Research (Sybesma, C., ed.), Vol. II, pp.423-426, Martinus Nijhoff/Dr. W. Junk, The Hague

Siggel, U., Renger, G., Steihl, H.H. and Rumberg, B. (1972) Evidence for electronic and ionic interaction between electron transport chains in chloroplasts. Biochim. Biophys. Acta 256, 328-335

Singer, S.J. and Nicolson, G.L. (1972) The fluid mosaic model of the structure of cell membranes. Science 175, 720-729

Slater, E.C. (1953) Mechanism of phosphorylation in the respiratory chain. Nature (London) 172, 975-978

Slater, E.C. (1973) The mechanism of action of the respiratory inhibitor, antimycin. Biochim. Biophys. Acta 301, 129-154

* Slater, E.C. (1974) In: Dynamics of Energy-Transducing Membranes (Ernster, L., Estabrook, R.W. and Slater, E.C., eds.), BBA Library, Vo1. 13, pp.1-20, Elsevier, Amsterdam 
Slater, E.C. (1983) The Q cycle, an ubiquitous mechanism of electron transfer. Trends Biochem. Sci. $\underline{8}, 239-242$

Smith, J.C., Russ, P., Cooperman, B.S. and Chance, B. (1982) Synthesis, structure determination, spectral properties and energy-linked spectral responses of the extrinsic probe oxonol $\mathrm{V}$ in membranes. Biochem. 15, 5094-5105

Steele, J.A., Uchytil, T.F., Durbin, R.D., Bhatnagar, P.K. and Rich, D.H. (1978) The stimulation of $\mathrm{CF}_{1}$ ATPase by tentoxin and its analogs. Biochem. Biophys. Res. Comm. 84, 215-218

Steinback, K.E., Burke, J.E. and Arntzen, C.J. (1979) Evidence for the role of the surface-exposed portion of the light-harvesting complex in cation-mediated control of chloroplast structure and function. Arch. Biochem. Biophys. 195, 546-557

Stiehl, H.H. and Witt, H.T. (1968) Die kurzzeltgen ultravioletten differenzspektren bie der photosynthese. Z. Naturforsch. 23B, 220-221

Strotmann, H., Bickel-Sandkotter, S. and Shoshan, V. (1979) Kinetic analysis of light-dependent exchange of adenine nucleotides on chloroplast coupling factor $\mathrm{CF}_{1}$ FEBS Lett. 101, 316-320

Tagawa, K. and Arnon, D.I. (1962) Ferredoxins as electron carriers in photosynthesis and in the biological production and consumption of hydrogen gas. Nature (London) 195, 537-543

Theg, S.M., Jurinsinc, P.A. and Homann, P.H. (1984) Studies on the mechanism of chloride action on photosynthetic water oxidation. Biochim. Biophys. Acta 766, 636-646

Thornber, J.P. (1975) Chorophyll-proteins: light harvesting and reaction centre components of plants. Ann. Rev. Plant Physiol. 26, 127-158

Tischer, W. and Strotmann, H. (1979) Some properties of the DCMU-binding site in chloroplasts. Z. Naturforsch. 34C, 992-995

Trebst, A. (1974) Energy conservation in photosynthetic electron transport of chloroplsats. Ann. Rev. Plant Physiol. 25, 423-458

Trebst, A. (1979) Inhibition of photosynthetic electron flow by phenol and diphenylether herbicides in control and trypsin treated chloroplasts. Z. Naturforsch. 34C, 986-991 
Vallon, 0., Wollman, F.A. and Olive, J. (1985) Distribution of intrinsic and extrinsic subunits of the PS II protein complex between appressed and non-appressed regions of the thylakoid membrane: An Immune cytochemical study. FEBS Lett. 183, 245-250

Van Grondelle, R. and Duysens, L.N. (1980) On the quenching of the fluorescence yield in photosynthetic systems. Plant Physiol. 65, $751-754$

Van Niel, C.B. (1941) The bacterial photosynthesis and their importance for the general problem of photosynthesis. Adv. Enzymol. $1,263-338$

Velthuys, B.R. (1978) A third site of proton translocation in green plant photosynthetic electron transport. Proc. Natl. Acad. Sci. U.S.A. 75, 6031-6034

Velthuys, B.R. (1979) Electron flow through plastoquinone and cytochromes $\underline{b}_{6}$ and $\underline{f}$ in chloroplasts. Proc. Nat1. Acad. Sci. U.S.A. 76, 2765-276 $\overline{9}$

Velthuys, B.R. (1980) Electron and proton transfer events in chloroplasts during a short series of flashes. FEBS Lett. 115, $167-170$

Velthuys, B.R. and Amesz, J. (1974) Charge accumulation at the reducing side of system 2 of photosynthesis. Biochim. Biophys. Acta $333,85-94$

Vredenberg, W.J. and Schapendonk, A.H.C.M. (1981) Reaction kinetics of P515 in chloroplasts: Transmembrane (Reaction I) and inner membrane (Reaction II) electric fields. In: Photosynthesis: Photophysical Processes - Membrane Energisation, (Akoyunoglou, G., ed.), Vol. I, pp.489-498, Balaban, Philadelphia

Wasielewska, M.R., Norris, J.R., Shipman, L.L., Lin, C.P. and Svec, W.A. (1981) Monomeric chlorophyll a enol: evidence for its possible role as the primary electron donor in photosystem I of plant photosynthesis. Proc. Nat1. Acad. Sci. U.S.A. 78, 2957-2961

Webster, G., Cogde1l, R.J. and Lindsay, J.G. (1980) Identification of the carotenoid present in the B800-850 antenna complex from Rhodopseudomonas capsulata as that which responds electrochemically to transmembrane fields. Biochim. Biophys. Acta 591, 321-330

Weiss, M.A. and McCarty, R.E. (1977) Cross-linking within a subunit of coupling factor 1 increases the proton permeability of spinach thylakoids. J. Biol. Chem. 252, 8007-8012 
Whitford, D., Gounaris, K. and Barber, J. (1984) Studies on cyt b-559 of the higher plant thylakoid membranes. In: Advances in

Photosynthesis Research (Sybesma, C., ed.), Vol. I, pp.497-500,

Martinus Nijhoff/Dr. W. Junk, The Hague

Whitmarsh, J. and Cramer, W.A. (1977) Kinetics of the photo-reduction of cyt b-559 by photosystem II in chloroplasts. Biochem. Biophys. Acta $46 \overline{0}, 280-289$

Whitmarsh, J. and Cramer, W.A. (1979) Photo oxidation of the high-potential iron-sulphur centre in chloroplasts. Proc. Natl. Acad. Sci. U.S.A. 76, 4417-4420

Wikstrom, M.K.F. (1973) The different cytochrome b components in the respiratory chain of animal mitochondria and their role in electron transport and energy conservation. Biochim. Biophys. Acta 301, 155-193

Willey, D.L., Auffret, A.D. and Gray, J.C. (1984) Structure and topology of Cyt $\underline{f}$ in pea chloroplast membranes. Cel1 $\underline{36}, 555-562$

Witt, H.T. and Zickler, A. (1974) Electrical evidence for the field indicating absorption change in bio-energetic membranes. FEBS Lett. 37, 307-310

Wolff, Ch., Buchwald, H.E., Ruppel, H., Witt, K. and Witt, H.T. (1969) Rise time of the light induced electric field across the functional membrane of photosynthesis. Z. Naturforsch. Teil B. 24, 1038-1041

Yamamoto, Y., Tabata, K., Isoeai, Y., Nishimura, M., Okayama, S., Natsuura, K. and Itoh, S. (1984) Quantitative analysis of membrane components in highly active $\mathrm{O}_{2}$ evolving photosystem II preparation from spinach chloroplasts. Biochim. Biophys. Acta 767, 493-500

Yocum, C.F., Yerkes, C.T., Blankenship, R.E., Sharp, R.R. and Babcock, G.T. (1981) Stoichiometry, inhibitor sensitivity and organisation of manganese associated with photosynthetic oxygen evolution. Proc. Nat1. Acad. Sci. U.S.A. 78, 7507-7511

Younis, H.M., Winget, G.D. and Racker, E. (1977) Requirement of the subunit of chloroplast coupling factor 1 photophosphorylation. J. Biol. Chem. 252, 1814-1818

Zhang, Z.D., Li, L.B., Tan, K.H., Zhai, X.J., Zhou, P.Z. (1980) Structural and functional analysis of chloroplast membrane using trypsin and chymotrypsin as structural modifiers. Acta. Bot. Sin. 22, 49-56 
Zimmerman, J.L. and Rutherford, A.W. (1985) The $\mathrm{O}_{2}$ evolving system of photosystem II. Recent advances. Physiol. Veg. 23, 1-10

* Denotes journals which were not available. 
Goldbeck, J.H. and Kok,B. (1979) Redox titration of electron acceptor $Q$ and the plastoquinone pool in photosystem II. Biochim. Biophys. Acta $547,347-360$

Gresser, M.J., Myers, J.A. and Boyer, P.D. (1982) Catalytic site cooperativity of beef heart mitochondrial $F_{1}$ adenosine triphosphatase. J. Biol. Chem. 257, 12030-12038

Jackson, J.B. and Dutton, P.L. (197). The kinetic and redox potentiometric resolution of the caretenoid shifts in Rhodopseudomonas sphaeroides chromatophores: their relationship to electric field alterations in electron transport and energy coupling. Biochim. Biophys. Acta $325,102-113$

Katoh, S., Shiratori, I. and Takamiya,A. (1962). Purification and some properties of spinach plastocyanin. J. Biochem. (Tokyo) 51, 32-40

Laemmli, U.K. (1970) Cleavage of structural proteins during the assembly of the head of bacteriophage T4. Nature (London) 227, 680-685

McCarty, R.E. and Racker, E. (1967) Partial restlution of the enzymes catalyzing photophosphorylation. J. Biol. Chem. 242,3425-3439

+ Mejbaum-Katzenellenbogen, S. and Drobryszyka, W.J. (1951) Clin. Chim. Acta $\underline{4}, 515-522$

Mills, J.D. and Mitchell, P. (1984) Thiol modulation of the chloroplast protonmotive ATPase and its effect on photophosphorylation. Biochim. Biophys. Acta 764, 93-104

Nelson, N., Nelson, H. and Schatz, G. (1980)Biosynthesis and assembly of the proton-translocating adenosine triphosphatase complex from chloroplasts. Proc. Natl. Sci. U.S.A. 77, 1361-1364

Pick, U. and McCarty, R.E. (1980) In: Methods in enzymology: Photosynthesis ana Nitrogen Fixation Part C (San Piętro, A., ed.), Vol 69, pp.538-546, Academic Press, New York

Stokes, D.M. and Walker, D.A. '1971) Phosphoglycerate as a Hill oxidant in a reconstituted chloroplast system. Plant Physiol. 48, 163-165

+ Title of article not available 
Additional reference list cont'd/

.

Wydrinski, T.J. (1982) In: Photosynthesis: Energy conversion by plants and bacteria (Govindjee, edis), Vol. I, pp.469-506, Academic Press, New York

Younis, H.M., Winget, D.G. and Racker, E. (1977) Requiremnt of the 8 sub-unit of chloroplast coupling factor 1 for phorphosphorylation. J. Biol. Chem. 252, 1814-1878 